\title{
EXTRACELLULAR VESICLES, LIPIDS, AND LIPOPROTEINS IN EARLY PREGNANT SHEEP
}

\author{
A dissertation submitted in partial fulfillment of \\ the requirements for the degree of \\ DOCTOR OF PHILOSOPHY \\ University of Missouri-Columbia \\ Division of Animal Science
}

By

ELEANORE V. O'NEIL

Dr. Thomas E. Spencer, Dissertation Supervisor

May 2021 
The undersigned, appointed by the dean of the Graduate School, have examined the dissertation entitled

\section{EXTRACELLULAR VESICLES, LIPIDS, AND LIPOPROTEINS \\ IN EARLY PREGNANT SHEEP}

presented by Eleanore O'Neil,

a candidate for the degree of doctor of philosophy, and hereby certify that, in their opinion, it is worthy of acceptance.

Chair, Dr. Thomas Spencer

Dr. Kevin Fritsche

Dr. Rodney Geisert

Dr. Thomas McFadden 


\section{ACKNOWLEDGEMENTS}

Never did I imagine I would be defending my dissertation during a pandemic! What a crazy time. This has been such a rewarding journey and I have cherished every moment here at Mizzou. I would like to acknowledge all the students, faculty, and staff for their support throughout my doctoral program. I am especially thankful to my research supervisor Dr. Thomas Spencer for giving me the opportunity to join his lab and for all the guidance and input he gave me. I would not be the scientist I am today without his mentorship. I also would like to acknowledge my committee members: Drs. Rod Geisert, Kevin Fritsche, and Thomas McFadden for their support and the wonderful discussions we had during my time here. I want to give a special shoutout to Dr. Geisert for his unwavering support of me.

I must extend the deepest gratitude for the members of Team Uterus, past and present: Drs. Kelsey Brooks, Joao Moraes, Andrew Kelleher, Pramod Dhakal, Kosta Simintiras, as well as Jacob Pearson, Wang Peng, Megan McClean, Clay Mitchell, and Jessica Milano-Foster. I consider Drs. M Sofia Ortega and Harriet Fitzgerald from the Spencer Lab to be both mentors and friends who really helped shape my academic journey. Most notably, Dr. Greg Burns and David Todd were my unending allies in the annual craziness that is Sheep Season. It has been a pleasure to call them my colleagues and friends. Finally, sincere appreciation and love for my fellow grad students, especially those in the Geisert Lab (Ashley Meyer, Caroline Pfeiffer, and Destiny Johns) and the Ortega Lab (Jason Rizo, Kelsey Clark, and Katy Stoecklein). They spent so many of their evenings at the barn, so 
many mornings at sheep surgeries, and plenty of afternoons laughing at lunch with me. It really takes a village.

Finally, to my crazy, loving family ('The Oneks')! My parents, Susan and Dennis, are my real-life heroes and my biggest cheerleaders, and my three older siblings, Iver, Lisabeth, and Stefan, and my future sister-in-law Shataakshi, are my best friends. My family never doubted me during all these years and even flew out to the middle of Missouri (leaving Florida, Washington, North Carolina, and New York) to spend Christmas with me and Will so I could stay with the sheep! It was worth it because two of our family Christmas photos featured us posing with sheep. And of course, thank you to my partner William Deng, who has been with me (and will be with me) through it all. I could have not asked for a better partner, and personally, I think he deserves a Ph.D. in sheep reproduction too! 


\section{TABLE OF CONTENTS}

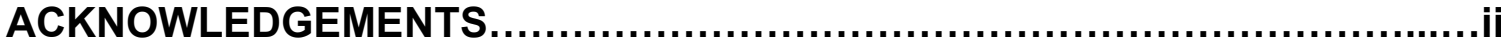

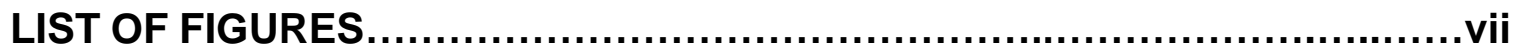

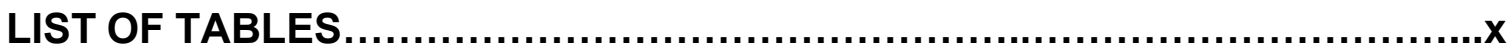

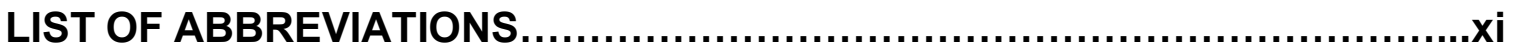

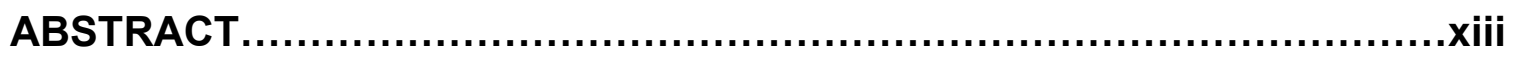

CHAPTER

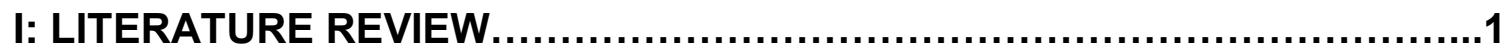

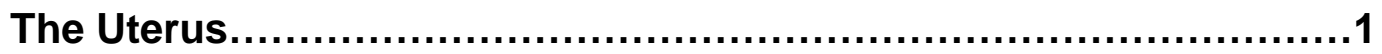

Uterine histoarchitecture...............................................



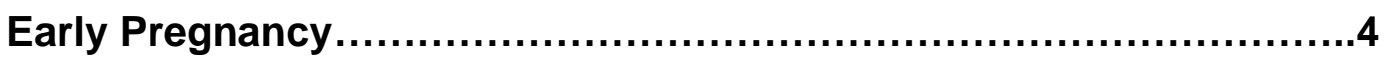

Conceptus development, implantation, \& placentation.................4

Maternal recognition signaling ............................................



Progesterone regulation of the endometrium..........................8

IFNT regulation of the endometrium....................................

Histotroph and uterine luminal fluid..................................10

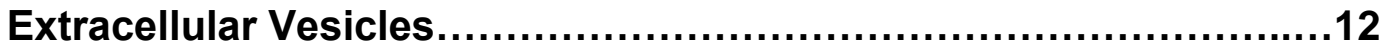

Biosynthesis of extracellular vesicles................................12

Extracellular vesicle cargo \& uptake...................................15

Extracellular vesicles in the uterine lumen.............................17

Metabolites and Lipids .....................................................

Metabolites in the uterine lumen.....................................22

Lipids in the uterine lumen...........................................24

Conceptus use of metabolites and lipids .............................27 


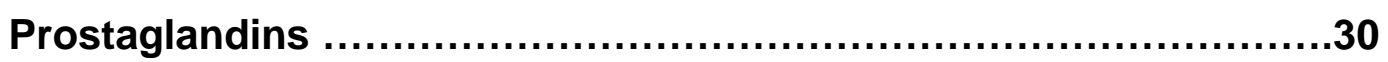

Biosynthesis of prostaglandins .....................................30

Prostaglandins in the uterine lumen..................................34

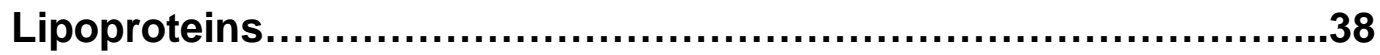

Biosynthesis of lipoproteins..........................................39

Lipoproteins and apolipoproteins in the uterine lumen................44

II: CHARACTERIZATION AND REGULATION OF EXTRACELLULAR VESICLES IN THE LUMEN OF THE OVINE UTERUS $\ldots \ldots \ldots \ldots \ldots \ldots \ldots \ldots \ldots \ldots . \ldots \ldots$

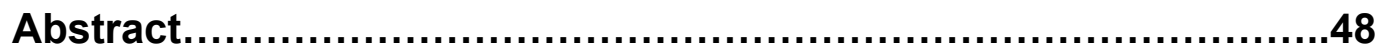

Introduction...................................................................

Materials and methods........................................................



Discussion............................................................... 71

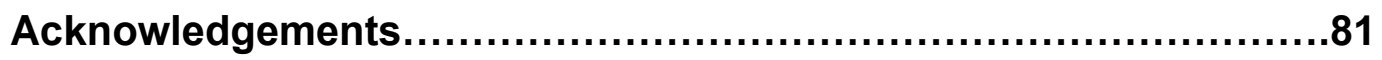

Supplemental information................................................82

III: EXTRACELLULAR VESICLE EFFECTS ON THE ENDOMETRIUM OF



Abstract....................................................................... 84



Materials and methods.........................................................

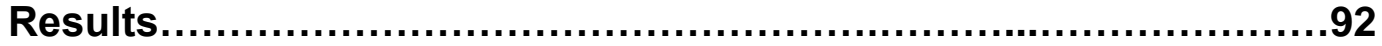

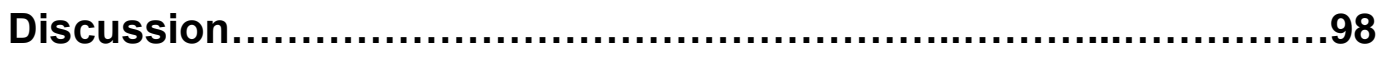

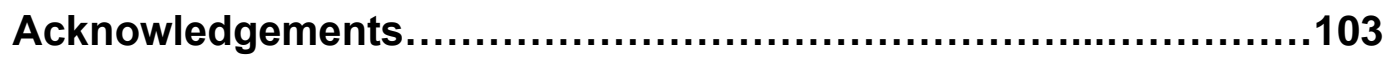

Supplemental information.................................................103

IV: INSIGHTS INTO THE LIPIDOME AND PRIMARY METABOLOME OF THE UTERUS FROM DAY 14 CYCLIC AND PREGNANT SHEEP....................104

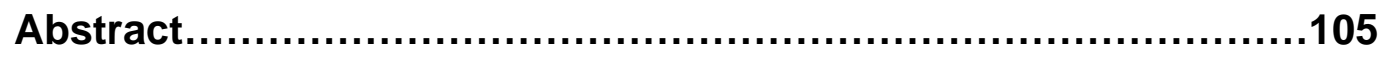



Materials and methods...................................................108 


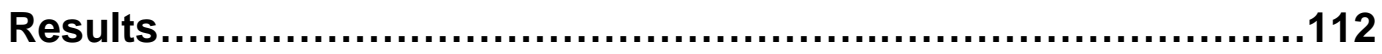

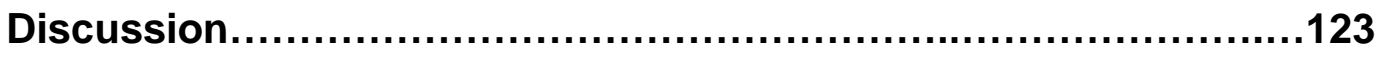



Supplemental information..........................................131

V: PROSTAGLANDIN-ENDOPEROXIDE SYNTHASE 2 IS NOT REQUIRED FOR PREIMPLANTATION OVINE CONCEPTUS DEVELOPMENT IN

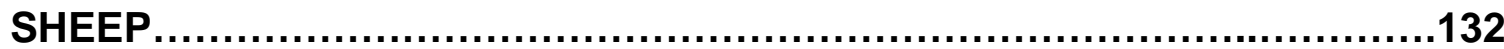

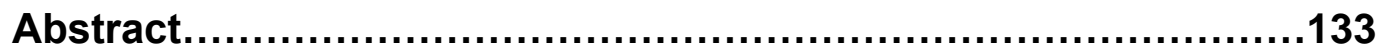

Introduction...............................................................134

Materials and methods ................................................136

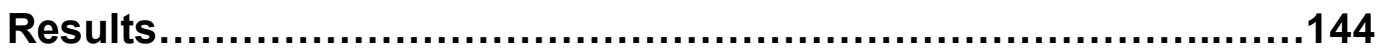

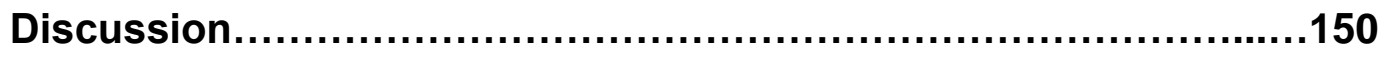

Acknowledgements............................................................

Supplemental information...........................................

VI: BIOLOGICAL ROLE OF APOA1 IN EARLY PREGNANCY IN SHEEP....156

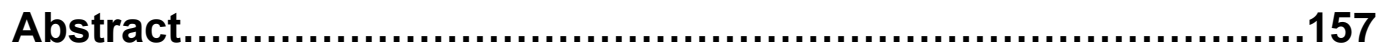

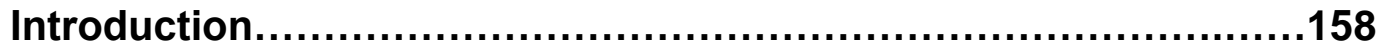

Materials and methods................................................160

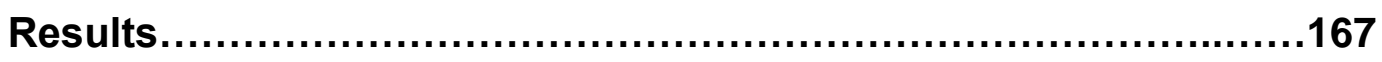

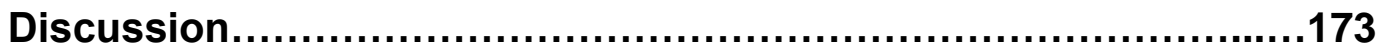

Acknowledgements......................................................177

Supplemental information..............................................177

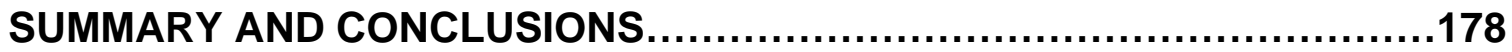

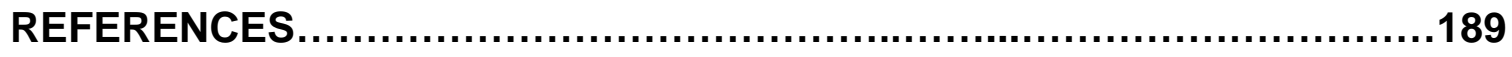

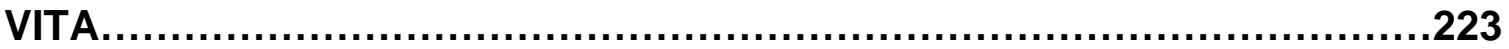




\section{LIST OF FIGURES}

\section{I: LITERATURE REVIEW}

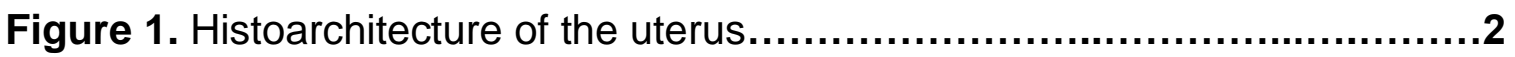

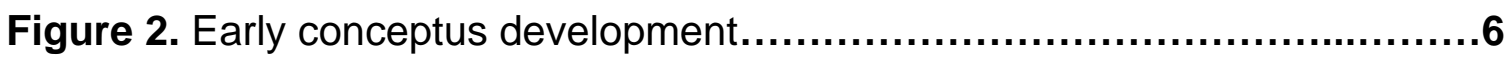

Figure 3. Regulation of the endometrial epithelium during early pregnancy......10

Figure 4. Synthesis, secretion, and uptake of extracellular vesicles...............14

Figure 5. Working hypothesis on the role of extracellular vesicles (EV) in early

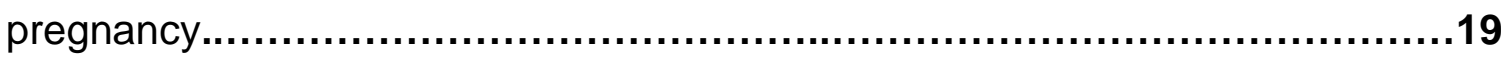

Figure 6. Schematic of prostaglandin (PG) biosynthesis.............................32

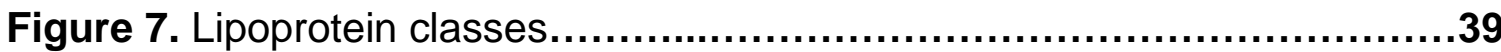

Figure 8. Lipoprotein interactions...............................................43

\section{II: CHARACTERIZATION AND REGULATION OF EXTRACELLULAR VESICLES IN THE LUMEN OF THE OVIEN UTERUS}

Figure 1. Extracellular vesicle (EV) number in the uterine lumen of day 14 cyclic and pregnant sheep.

Figure 2. Proteomic analysis of extracellular vesicles (EV) isolated from the uterine lumen of day 14 cyclic and pregnant sheep.

Figure 3. Phospholipids in extracellular vesicles (EV) isolated from the uterine lumen of day 14 cyclic and pregnant sheep

Figure 4. Ceramides, acylcarnitines, and free fatty acids (FFAs) in extracellular vesicles $(E V)$ isolated from the uterine lumen of day 14 cyclic and pregnant sheep.

68

Figure 5. Effect of extracellular vesicles $(E V)$ isolated from the uterine lumen of day 14 cyclic and pregnant sheep on ovine trophectoderm (oTr) cell proliferation

Figure 6. Effect of extracellular vesicles $(E V)$ isolated from the uterine lumen of day 14 cyclic and pregnant sheep on IFNT production by ovine trophectoderm (oTr) cells. 


\section{III: EXTRACELLULAR VESICLE EFFECTS ON THE ENDOMETRIUM OF SHEEP}

Figure 1. Summary of endometrial transcriptome analyses......................93

Figure 2. Enrichment analysis of differentially expressed transcripts in CEV infused ewes

Figure 3. Prostaglandins were elevated in the uterine lumen of CEV infused ewes compared to PEV infused ewes. .96

Figure 4. Infusion of PEV prevented upregulation of uterine ESR1 .97

\section{IV: INSIGHTS INTO THE LIPIDOME AND PRIMARY METABOLOME OF THE UTERUS FROM DAY 14 CYCLIC AND PREGNANT SHEEP}

Figure 1. Triglycerides (TAG) in the endometrium from day 14 cyclic and pregnant sheep 114

Figure 2. Triglycerides (TAG) in the uterine lumen..............................115

Figure 3. Phospholipids in the uterine lumen...................................116

Figure 4. Principal component analysis (PCA) of the conceptus, endometrial, and uterine lumen flush (ULF) lipidome. 118

Figure 5. Enrichment analysis of metabolites elevated in the uterine lumen of day 14 cyclic (14C) and pregnant (14P) sheep.

Figure 6. Amino acids in the uterine lumen 122

Figure 7. Principal component analysis of the day 14 sheep conceptus, endometrium, and uterine lumen (ULF) metabolome.

122

\section{V: PROSTAGLANDIN-ENDOPEROXIDE SYNTHASE 2 IS NOT REQUIRED FOR PREIMPLANTATION OVINE CONCEPTUS DEVELOPMENT IN SHEEP}

Figure 1. Structure and targeting of the Ovis Aries PTGS2 gene.................144

Figure 2. Design and effect of CRISPR gRNAs targeting the ovine PTGS2 for genomic editing.

Figure 3. Effect of PTGS2 editing on PTGS2 protein and prostaglandin (PG) production

Figure 4. Effects of PTGS2 edited embryos on endometrial gene expression and gene expression in PTGS2 edited embryos. 


\section{VI: BIOLOGICAL ROLE OF APOA1 IN EARLY PREGNANCY IN SHEEP}

Figure 1. Effects of APOA1 infusion on epithelial lipid droplets and gene



Figure 2. Heatmap analysis of TG in the ULF of vehicle infused (green) and APOA1 infused (red) sheep......................................................168

Figure 3. APOA1 gene structure and CRISPR-Cas9 gene editing effects.......170

Figure 4. Effects of APOA1 editing on placental gene expression in APOA1 targeted conceptuses....................................................................

Figure 5. Effects of APOA1 editing on fetal and placental development.........172 


\section{LIST OF TABLES}

\section{II: CHARACTERIZATION AND REGULATION OF EXTRACELLULAR VESICLES IN THE LUMEN OF THE OVIEN UTERUS}

Table 1. Ten most abundant proteins in EVs from the uterine lumen of

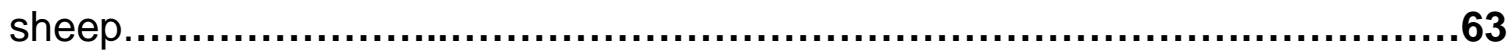

Table 2. Top 10 differentially abundant proteins in EVs isolated from the uterine lumen of day 14 cyclic and pregnant sheep...........................................64

\section{IV: INSIGHTS INTO THE LIPIDOME AND PRIMARY METABOLOME OF THE UTERUS FROM DAY 14 CYCLIC AND PREGNANT SHEEP}

Table 1. Individual ceramide and NEFA lipids altered in the uterine lumen of day 14 cyclic $(C)$ and pregnant $(P)$ sheep. 117

Table 2. Total lipids in the conceptus, endometrium and uterine lumen on day 14 of pregnancy in sheep.

Table 3. Top 10 metabolites more abundant in the uterine lumen of day 14 pregnant $(P)$ than cyclic $(C)$ sheep

Table 4. Top 10 metabolites less abundant in the uterine lumen of day 14 pregnant



\section{III: PROSTAGLANDIN-ENDOPEROXIDE SYNTHASE 2 IS NOT REQUIRED FOR PREIMPLANTATION OVINE CONCEPTUS DEVELOPMENT IN SHEEP}

Table 1. Effect of PTGS2 gene editing on conceptus development and uterine PGs and IFNT 


\section{LIST OF ABBREVIATIONS}

$\begin{array}{ll}\text { AA } & \text { Arachidonic acid } \\ \text { APOA1 } & \text { Apolipoprotein A1 } \\ \text { BNC } & \text { Giant binucleate cells } \\ \text { CE } & \text { Cholesteryl esters } \\ \text { CL } & \text { Corpus luteum } \\ \text { DAG } & \text { Diacylglycerols } \\ \text { E2 } & \text { Estrogen } \\ \text { enJSRV } & \text { Endogenous Jaagsiekte sheep retrovirus } \\ \text { ESCRT } & \text { Endosomal sorting complexes required for transport } \\ \text { FA } & \text { Fatty acid } \\ \text { FSH } & \text { Follicle-stimulating hormone } \\ \text { GE } & \text { Glandular epithelium } \\ \text { GnRH } & \text { Gonadotropin-releasing hormone } \\ \text { HDL } & \text { High-density lipoproteins } \\ \text { IDL } & \text { Intermediate-density lipoproteins } \\ \text { IFNT } & \text { Interferon tau } \\ \text { ILV } & \text { Intraluminal vesicles } \\ \text { ISG } & \text { Interferon stimulated genes } \\ \text { LDL } & \text { Low-density lipoproteins } \\ \text { LE } & \text { Luminal epithelium } \\ \text { LH } & \text { Luteinizing hormone } \\ \text { LPA } & \text { Mysophosphatidic acid } \\ \text { MAG } & \text { Mamoacylglycerols } \\ \text { MTOR } & \text { Microvesicles } \\ \text { MV } & \text { MVBivesicular bodies } \\ \text { MV } & \end{array}$




$\begin{array}{ll}\text { NEFA } & \text { Non-esterified fatty acids } \\ \text { P4 } & \text { Progesterone } \\ \text { PC } & \text { Phosphatidylcholine } \\ \text { PE } & \text { Phosphatidylethanolamine } \\ \text { PG } & \text { Prostaglandin } \\ \text { PGL } & \text { Phosphatidylglycerol } \\ \text { PI } & \text { Phosphatidylinositol } \\ \text { PS } & \text { Phosphatidylserine } \\ \text { TAG } & \text { Triacylglycerols } \\ \text { TCA } & \text { Tricarboxylic acid } \\ \text { TE } & \text { Trophectoderm } \\ \text { UGKO } & \text { Uterine gland knockout } \\ \text { ULF } & \text { Uterine luminal fluid } \\ \text { VLDL } & \text { Very low-density lipoproteins }\end{array}$




\begin{abstract}
In sheep, pregnancy establishment encompasses conceptus elongation, implantation, and placentation. These events are regulated by factors present within the uterine luminal fluid (ULF) from the endometrial epithelium and the conceptus itself that affect proliferation, migration, attachment, and adhesion of the conceptus trophectoderm. As the peri-implantation period is especially susceptible to pregnancy loss, it is essential to understand the various components and functional roles of substances within the ULF. The central hypothesis of this dissertation is that lipids and lipid associated macromolecules are components of the ULF and mediate endometrial-embryonic crosstalk and regulate conceptus development. This work sought to identify, characterize, and/or determine the roles of: (1) extracellular vesicles (EVs); (2) lipids and metabolites; (3) prostaglandins (PGs); and (4) apolipoproteins present within the ULF of ewes during early gestation. Collectively, the present studies established that: (1) EVs increase within the ULF during the estrous cycle but are depleted in the uterine lumen of pregnant ewes due to uptake by the elongating conceptus; (2) the lipid and protein cargo of uterine EVs is diverse and altered by pregnancy; (3) uterine EVs regulate cellular processes in the conceptus trophectoderm and endometrium including cell proliferation and secretions; (4) various lipids (specifically phospholipids, ceramides, and triglycerides) and metabolites are elevated in the ULF of pregnant ewes; (5) the conceptus lipidome and metabolome is distinct from the ULF and endometrium suggesting selective uptake of ULF substances; (6) the production of PGs by PTGS2 in the conceptus is not required for conceptus elongation; (7)
\end{abstract}


the secretion of APOA1 by the conceptus does not mobilize endometrial lipids into the ULF and is not required for early pregnancy development or survival. Collectively, these studies highlight the complex and dynamic composition of the ULF and support the overall hypothesis that lipids and lipid-associated macromolecules are critical components of the ULF that mediate conceptusendometrial crosstalk and regulate important developmental processes in the conceptus. Future investigation and expansion of these findings will fill crucial gaps in our knowledge of early pregnancy events and may provide biomarkers or help develop therapies to improve pregnancy outcomes and reproductive efficiency in agricultural species. 


\section{CHAPTER I:}

\section{LITERATURE REVIEW}

\section{The Uterus}

\section{Uterine histoarchitecture}

The mammalian uterus contain three distinct layers: (1) the perimetrium, the serous outer layer; (2) the myometrium, the middle layer composed of an inner circular and outer longitudinal smooth muscle layer; and (3) the endometrium, the innermost functional layer of the uterus that comes into direct contact with the developing embryo during gestation. The endometrium contains luminal epithelium (LE), glandular epithelium (GE), stroma (stratum compactum and stratum spongiosum), blood vessels, and immune cells (Figure 1). In sheep, the endometrium is organized into approximately 80 regions of densely packed aglandular stromal protrusions called caruncles surrounded by intercaruncular regions containing hundreds of uterine glands per cross section [1]. Caruncles serve as the site for placentation in ewes after partial fusion with fetal cotyledons [2]. The ovine bicornuate uterus develops in utero from the paramesonephric, or Müllerian, ducts [3]. While most of the female reproductive tract and the outer two layers of the uterus have finished developing by the time of parturition, the endometrium is undifferentiated mesenchyme lined with a simple LE at birth $[4,5]$. Postnatal differentiation of the endometrium in sheep primarily involves the emergence and proliferation of GE and growth of the caruncles $[6,7]$. 


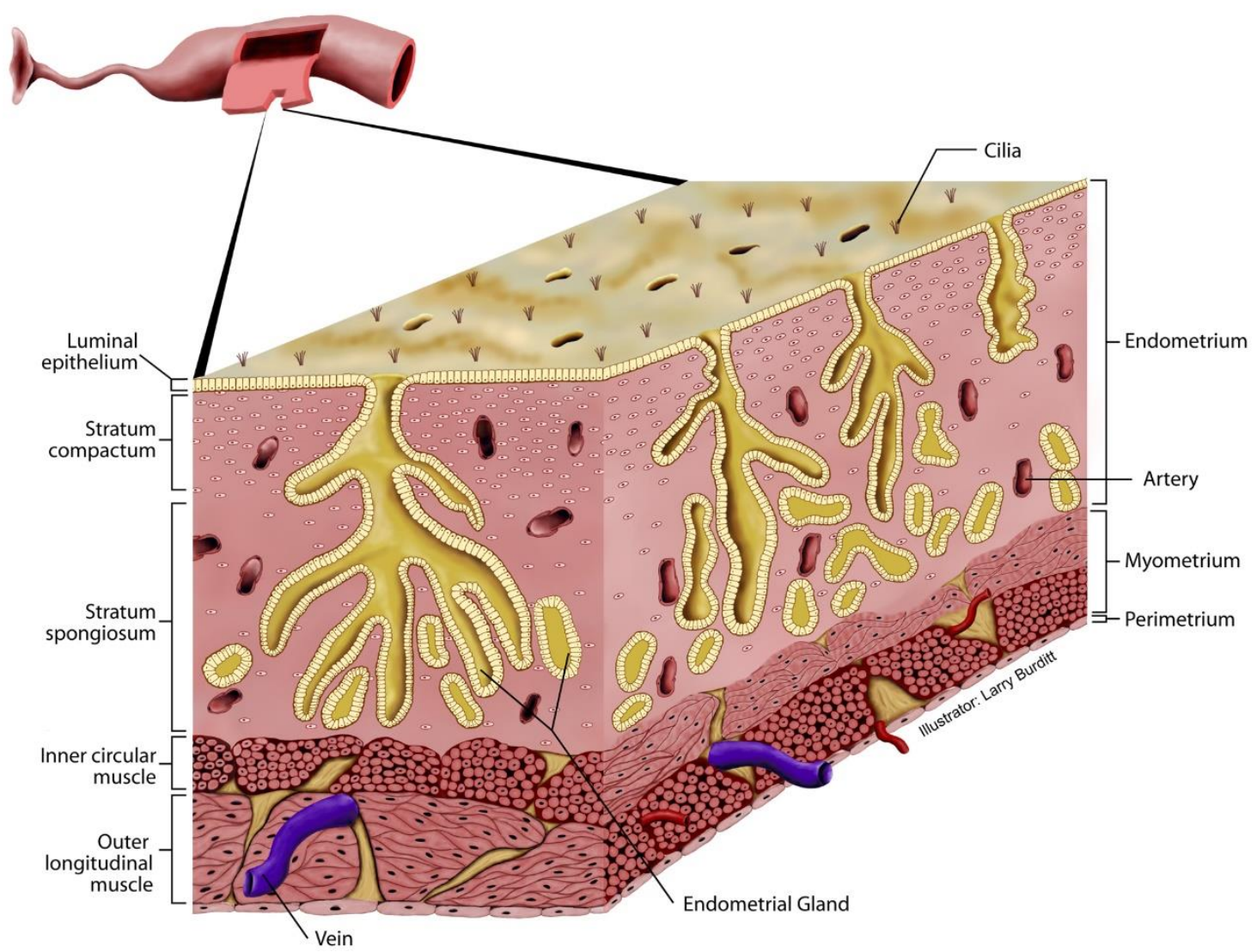

Figure 1. Histoarchitecture of the uterus. The mammalian uterus is composed of the perimetrium, the myometrium, and the endometrium, which is the innermost functional layer of the uterus. The endometrium is heavily vascularized and is composed of luminal epithelium (LE) and glandular epithelium (GE) that invaginate underlying stromal cells. Figure courtesy of Rodney Geisert and Larry Burdett, Oklahoma State University, Stillwater, USA).

\section{Estrous Cycle}

The estrous cycle lasts approximately 17 days in the ewe. Estrus, or the period the ewe will accept the ram for mating, defines the start of each estrous cycle (estrus = day 0 ) and lasts approximately $24-36$ hours. High levels of estrogen (E2) from the dominant pre-ovulatory follicle drive mating behavior and cause a surge in gonadotropin releasing hormone $(\mathrm{GnRH})$ from the hypothalamus. The GnRH surge induces downstream surges in luteinizing hormone $(\mathrm{LH})$ and follicle stimulating hormone (FSH) from the pituitary gland [8]. The LH surge causes 
ovulation approximately 30-36 hours after estrus and initiates luteinization of the ovulated follicle. Luteinization transforms follicular cells into steroid producing luteal cells that produce progesterone (P4) [9]. High levels of P4 from the corpus luteum (CL) exert negative feedback on $\mathrm{GnRH}$ production which alters the basal secretory pattern of $\mathrm{LH}$ and $\mathrm{FSH}$ and blocks expression of estrogen receptor alpha (ESR1) in the endometrium. Basal levels of FSH stimulate recruitment and early follicular growth in waves throughout the estrous cycle, but preovulatory follicles cannot fully develop until the CL stops producing P4. After approximately ten days of sustained P4 production from the $\mathrm{CL}$, progesterone receptor (PGR) is exclusively downregulated in the endometrial epithelium [10]. Loss of PGR and P4 signaling in the epithelium upregulates epithelial ESR1 on day 13 of the estrous cycle. E2 produced by the developing ovarian follicles binds to ESR1 in the uterine epithelium and upregulates oxytocin receptor (OXTR), likely by binding SP1 elements upstream of the OXTR promoter [11]. Oxytocin is produced from the CL and the posterior pituitary gland and activation of its receptor causes the generation of four or five luteolytic pulses of prostaglandin F2 $\alpha$ (PGF2 $\alpha$ ) from the endometrium [12-14]. PGF2 $\alpha$ is transported to the ovary and activation of its receptors on the luteal cells causes structural and functional regression of the CL $[15,16]$. With low levels of $\mathrm{P} 4$, negative feedback on $\mathrm{GnRH}$ is lifted and follicles can develop to the preovulatory stage due to $\mathrm{GnRH}$-induced shifts in tonic $\mathrm{LH}$ secretion and selection of the next dominant follicle. Rising E2 production by the dominant follicle initiates a new estrous cycle and another opportunity to breed. 


\section{Early Pregnancy}

Conceptus development, implantation, and placentation

The initial steps of early embryo development are similar between most mammalian species. After oocyte fertilization and initial mitotic divisions in the oviduct, the 8-16 cell ovine embryo, or morula, undergoes the maternal to zygotic transition and activates its own genome [17]. The 16-32 cell morula undergoes a process of compaction whereby cells on the exterior form gap junctions and desmosomes that pump fluid inside and form a central cavity called the blastocoel. This process leads to the formation of the blastocyst which contains a surrounding monolayer of trophectoderm (TE) cells that will give rise to the placenta, and an inner cell mass (ICM) that resides in the blastocoel and develops into the embryo proper [18].

In sheep and cattle, the embryo enters the uterus around day 6 (day $0=$ mating) as a morula or an early blastocyst [19]. Around day 8, the spherical blastocyst sheds its zona pellucida, a thick glycoprotein membrane that surrounds the embryo, and expands into a large spherical conceptus (embryo and its associated extraembryonic membranes). Unlike other species, domestic ruminants and pigs have an extended peri-implantation period with only superficial contact with the endometrium. During this period, the conceptus TE elongates over a series of days. Initially only $400-900 \mu \mathrm{m}$ in diameter on day 10 , the conceptus elongates to more than $25 \mathrm{~cm}$ in length by day 17 and extends from the uterine horn ipsilateral to the CL to the contralateral horn [20]. These morphological changes are driven by cellular proliferation and hypertrophy with a 60 -fold increase 
in tissue weight [21]. During conceptus elongation, cytoplasmic projections of the TE cells closely interdigitate with microvilli on the apical side of the LE [20]. Between days 15 and 18, the TE develop papillae which penetrate into the mouths of uterine glands and anchor the conceptus [22]. Firm adhesion of the TE to the LE begins on day 16 and involves integrins and select adhesion molecules such as osteopontin (SPP1), galectin-15 (LGALS15), and glycosylation-dependent cell adhesion molecule-1 (GlyCAM-1) [23-27]. Adhesion starts near the ICM of the conceptus and spreads to either ends of the conceptus until completion by day 22 (Figure 2). 


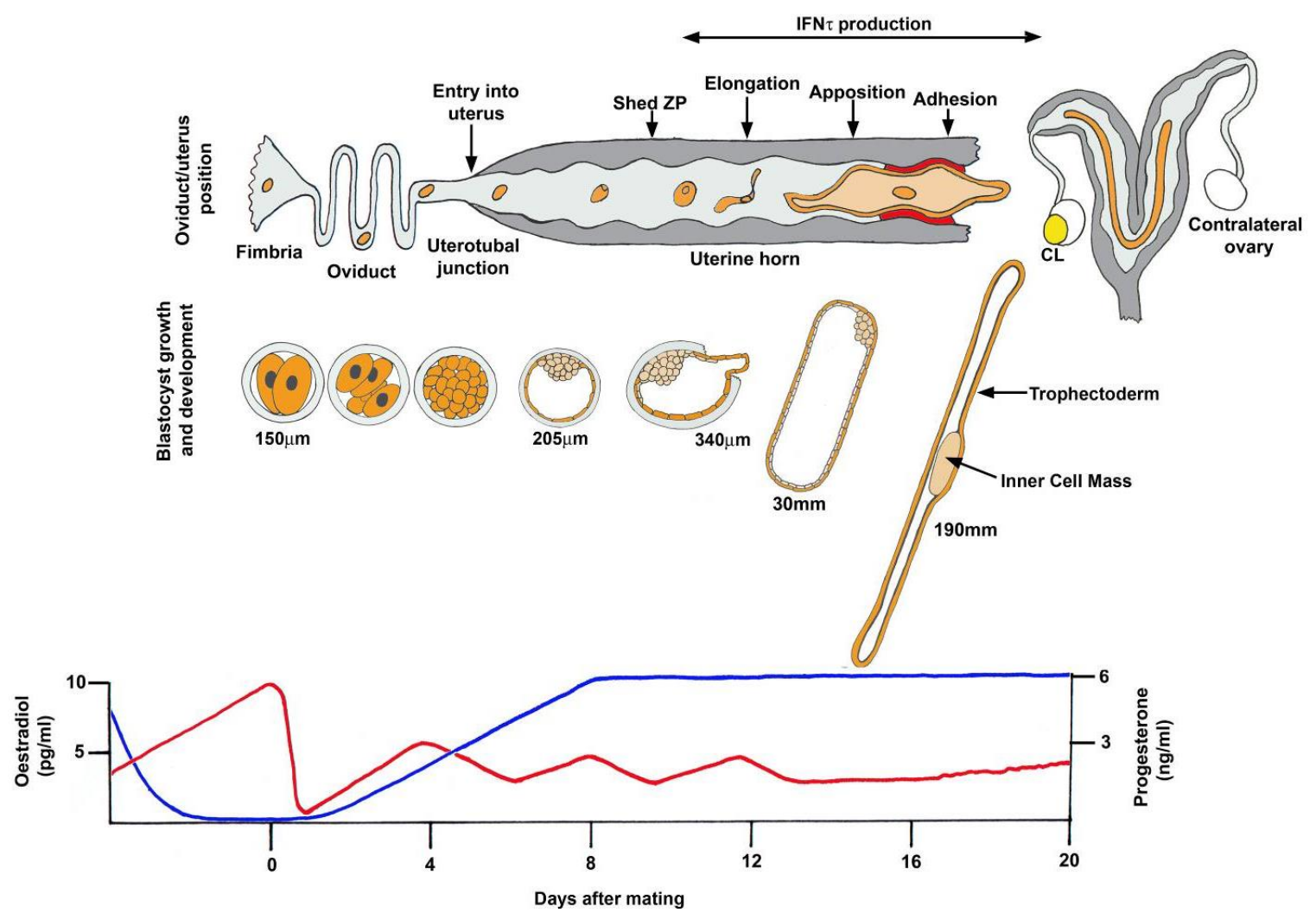

Figure 2. Early conceptus development. After fertilization and initial cleavages of the embryo in the oviduct, the embryo enters the uterus as a morula or early blastocyst around day 6 postmating. On day 8 , the blastocyst hatches from the zona pellucida and expands into a spherical conceptus (embryo and its associated extraembryonic membranes). Between days 10 and 12, the ovine conceptus begins to elongate into an ovoid or tubular shape and produces substantial quantities of interferon tau (IFNT), the maternal recognition signal of pregnancy. By day 14, the conceptus is approximately $10 \mathrm{~cm}$ long and apposes itself to the luminal epithelium. Firm adhesion to the endometrium begins on day 16 , when the conceptus is more than $15 \mathrm{~cm}$ in length and stretches from the uterine horn ipsilateral to the $\mathrm{CL}$ to the contralateral horn. Figure from [28].

The primitive streak is present on the conceptus by day 14 and marks the start of gastrulation as various subpopulations (e.g. epiblast, hypoblast, primitive mesoderm) that give rise to the germ layers differentiate $[20,29,30]$. During this process, subpopulations of the primitive mesoderm cells migrate to opposite poles to either fuse with the primitive endoderm and form the yolk sac or fuse with the 
TE to form the chorion. Meanwhile, trophoblast giant binucleate cells (BNC) begin to differentiate from mononuclear TE on day 16 [31-33]. Eventually BNC migrate across the fetal-maternal interface to fuse with and erode caruncular LE cells and form trinucleated feto-maternal hybrid cells or multinucleated syncytial plaques $[32,34]$. These syncytial plaques eventually line and invade the surface of the endometrial caruncles and, supported by mesenchymal chorionic cells, form fetal cotyledons that interdigitate with maternal caruncles to form placentomes [2]. In total, around 80 placentome units form across the placental surface area supported by extensive capillary beds [35, 36]. Placentomes continue to vascularize over the 147-day gestation period. Meanwhile, fetal chorion and maternal LE layers in the intercaruncular areas of the endometrium do not come into intimate contact but remain adjacent to each other throughout gestation [2].

\section{Maternal recognition signaling}

For successful pregnancy establishment, the developing conceptus must produce a maternal recognition of pregnancy signal to prevent luteolysis of the $\mathrm{CL}$ prior to day 16. In sheep and cattle, the maternal recognition signal is interferon tau (IFNT) [37-39]. IFNT is a secreted Type I IFN made of 172 amino acids that binds the IFN receptors IFNAR1 and IFNAR2 [37, 40, 41]. Expression of IFNT is detectable at the blastocyst stage and continues throughout conceptus elongation with maximal production between days 14 to $16[42,43]$. To prevent luteolysis, IFNT prevents the upregulation of epithelial ESR1 on day 13 through interferon regulatory factor 2 (IRF2) action [11, 44, 45]. Without epithelial expression of ESR1, OXTR is not expressed and the endometrium does not generate luteolytic 
pulses of PGF2 $\alpha$, thereby maintaining the $\mathrm{CL}$ and $\mathrm{P} 4$ production for establishment of pregnancy [46].

\section{Regulation of the Uterine Environment}

Progesterone regulation of the endometrium

Progesterone is essential for pregnancy maintenance, as withdrawal of P4 at any point during gestation results in pregnancy loss. P4 does not directly affect the conceptus, but rather stimulates endometrial gene expression changes and endometrial functions that support conceptus elongation and pregnancy establishment [47-49]. Loss of PGR in the epithelium substantially alters epithelial gene expression with a notable induction and upregulation of elongation and implantation associated genes. In the LE, P4 induces genes of secreted attachment and migration factors [galectin 15 (LGALS15) and insulin like growth factor (IGFBP1)], proteases and protease inhibitors [cathepsin L (CTSL), cystatins (CST3 and CST6)], transcription factors [hypoxia inducible factor $1 \mathrm{~A}($ HIF1A) and HIF2A], and intracellular enzymes [hydroxysteroid dehydrogenase 11 B1 (HSD11B1) and prostaglandin synthase 2 (PTGS2)] [50]. In the GE, P4 upregulates the proliferation regulating gastrin releasing peptide (GRP) and the secreted adhesion factor osteopontin (SPP1) [51]. Furthermore, P4 also causes a transient loss of various claudin and occludin proteins that make up tight and adherens junctions in the epithelial cells, potentially affecting serum and tissue fluid transudation into the uterine lumen [52]. 
IFNT regulation of the endometrium

Although most notable for its antiluteolytic actions, IFNT and other secreted factors by the conceptus work synergistically with P4 to modify gene expression in the endometrium. Expression of IRF2 prevents the expression of classical interferon stimulated genes (ISGs) in the uterine LE and superficial GE [53, 54]. However, IFNT upregulates various elongation and implantation related genes already stimulated by P4, such as LGALS15 and CTSL [55-57]. These genes are considered 'non-classical' ISG in the endometrial epithelium. In contrast, IRF2 is not expressed in the stroma or deep GE, and classical ISGs such as interferon stimulated gene 15 (ISG15), interferon induced GTP-binding protein Mx1 (Mx1), and 2',5'-oligoadenylate synthetase (OAS) are induced by IFNT [58-61]. These actions are likely mediated by dimerization of the signal transducers and activators of transcription (STAT) proteins STAT1 and STAT2 into the transcription factor complexes gamma-activated factor (GAF) and ISG factor 3 (ISGF3) which regulate expression of IFN regulatory factors (IRF) IRF1 and IRF9 and other ISGs through binding to IFN stimulated response elements (ISRE) in gene promoters [54, 62, 63]. IFNT is a prime example of how the conceptus produces and secretes substances that act on the endometrium and modify the uterine environment to regulate its own growth and development (Figure 3). 
TROPHECTODERM



Days 4-10

Days 11-12

Day 14 \& After

Figure 3. Regulation of the endometrial epithelium during early pregnancy. After sustained P4 production from the $\mathrm{CL}$, progesterone receptor (PGR) is exclusively downregulated in the endometrial epithelium between days 11 and 12 of pregnancy. The loss of PGR is coincident with the induction of many genes that regulate conceptus elongation and implantation. Production of interferon tau (IFNT), prostaglandins (PG), and cortisol by the conceptus trophectoderm and endometrial PG work synergistically with P4 to further upregulate (as indicated in bolded gene names) gene expression in the luminal (LE) and glandular epithelium (GE) by day 14. Many of these endometrial genes alter the uterine luminal fluid (ULF) and promote conceptus proliferation, migration, and attachment. Figure from [55].

Histotroph and uterine luminal fluid

The intrauterine environment is essential for pregnancy establishment. While blastocysts can form in vitro and hatch from the zona pellucida, they must be transferred to a synchronized recipient uterus to undergo conceptus elongation 
$[64,65]$. Studies using the uterine gland knockout (UGKO) ewe further demonstrated that secretions from the endometrial epithelium regulate early embryonic development [66]. Treatment of postnatal ewes with a nonmetabolizable progestin permanently ablates the development of uterine glands; although UGKO ewes ovulate competent oocytes that can be fertilized and develop to the blastocyst stage, blastocysts fail to elongate in the UGKO uterus $[67,68]$. Collective studies demonstrate how P4 and IFNT induce changes in endometrial gene expression that alter epithelial secretions, or histotroph, and drive changes in uterine luminal fluid (ULF) to promote conceptus development $[57,59,69]$. Overall, The ULF contains a complex mixture of molecules from the endometrium, conceptus, and serum that create a custom uterine environment conducive for conceptus elongation [70, 71]. Like IFNT, many of these factors mediate embryonic-endometrial communication at a time that the conceptus and endometrium are not yet in intimate contact. The protein components of the ruminant ULF (e.g. IFNT, LGALS15, CTSL, SPP1) have been extensively studied and characterized in pregnancy [72-75]. However, many other molecules including extracellular vesicles, metabolites, lipids, prostaglandins, and lipoproteins are present in the ULF and may play critical roles in conceptus elongation or survival. Thus, more studies are needed to better characterize their presence, regulation, functions, and overall importance in early pregnancy events. 


\section{Extracellular Vesicles}

Extracellular vesicles, or EV, are a heterogenous population of membrane bound vesicles that are secreted into the extracellular space and can be internalized by recipient cells. They are produced by most cell types in the body and are found in almost every biofluid. As the various vesicle subtypes are difficult to isolate and distinguish from each other, the International Society of Extracellular Vesicles has recommended using the term EVs to refer to the mixture of both exosomes and microvesicles [76]. Growing evidence suggests that EVs are mediators of intercellular communication in many biological systems as their cargo, which includes lipids, protein, and nucleic acids (such as mRNAs and miRNAs) can affect recipient cell function or biology.

\section{Biosynthesis of extracellular vesicles}

Exosomes are small, plasma membrane bound vesicles that range from 50 to $150 \mathrm{~nm}$ and are formed when intraluminal vesicles (ILV) bud into the lumen of multivesicular bodies (MVB) within the endosomal network [77-80]. The endosomal sorting complexes required for transport (ESCRT) protein complexes drive formation of ILV by recruiting and clustering proteins with ubiquitin and ubiquitin-like modifications onto the MVB membrane and promoting the inward budding and fission of these microdomains into the MVB [81, 82]. Intraluminal budding of endosomal membranes is supported by the interactions of syndecan, syntenten, and ALIX proteins [83]. Interestingly, ISG15 inhibits the formation of MVB and is highly induced by IFNT in the stroma of pregnant ewes [81]. Thus, production of IFNT by the conceptus may regulate exosome release by the 
endometrium. There are also ESCRT-independent pathways for MVB formation, in which self-organizing protein domains accumulate on ceramide rich lipid rafts and GPI-anchored proteins cause invaginations of the cell plasma membrane forming MVBs that can eventually release exosomes [84]. As part of the endosomal network, MVB must be directed for release into the extracellular space to avoid degradation in the lysosome. This process is mediated by the interaction of kinesin and RAB27b with cellular microtubules [85]. Notably, MVB destined for secretion in polarized epithelial cells typically migrate toward the apical pole of the cell for exosome release [78, 80]. Release of exosomes happens after MVB fuse with the cell plasma membrane and release the ILV in a SNARE and synaptotagmin-dependent process [86]. The alternative strategies for exosome formation and release underlie the formation of distinct subpopulations of exosomes. Overall, exosome secretion is a tightly regulated process that involves specific targeting of MVB in the endocytic pathway to be redirected away from lysosomal degradation and for secretion into the extracellular space.

In contrast with exosomes, microvesicles (MV) are large vesicles that range from 100 to $1000 \mathrm{~nm}$ and bleb directly off the cell membrane. The mechanisms required for $\mathrm{MV}$ formation and release are not fully understood but involves organization of lipid rafts on the plasma membrane and localized enrichment of phosphatidylserine (PS) and PE phospholipids. Phospholipid scramblase enzymes play crucial roles in transporting PS phospholipids to the outer membrane, which induces curvature and bending of the plasma membrane [87]. Cargo targeting to microvesicles is thought to occur due to affinity of proteins for 
lipid rafts, leading to relatively distinct makeup from that of exosomes. Additionally, plasma membrane associated cargo are more likely to be enriched in microvesicles than exosomes. Final microvesicle fission and release into the extracellular space is regulated by Ras-related GTPase ADP-ribosylation factor 6 (ARF6) and Rho GTPase-dependent phosphorylation of myosin chains which stimulates rearrangement of actin and membrane contraction [88]. Microvesicles release may be a faster process than exosome release, since exosomes require recruitment and organization within the endosomal network (Figure 4).

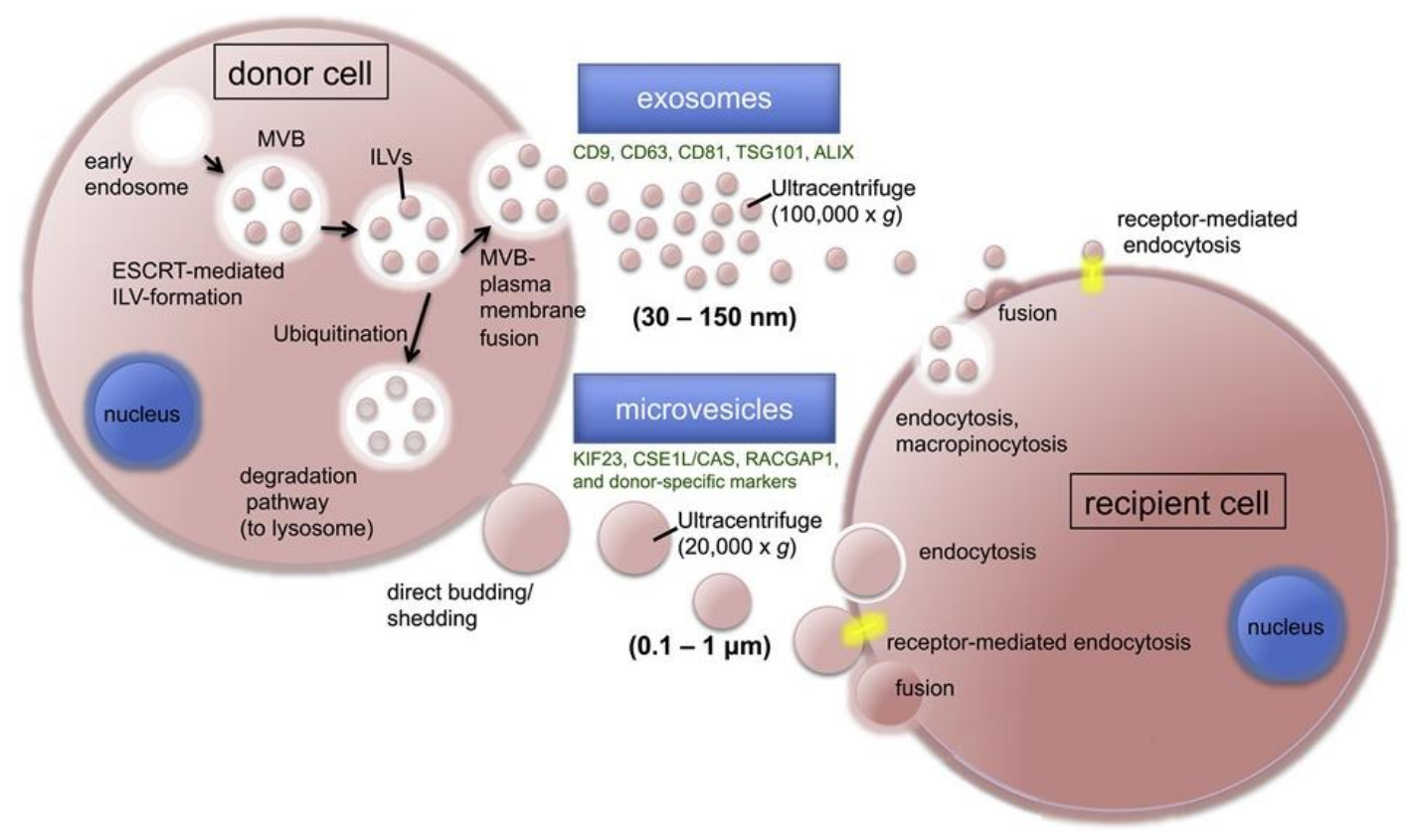

Figure 4. Synthesis, secretion, and uptake of extracellular vesicles. Extracellular vesicles (EV) refer to a heterogenous population of exosomes and microvesicles released into the extracellular space that contain protein, lipid, and RNA cargo. Exosomes form as intraluminal vesicles (ILV) within multi-vesicular bodies (MVB) of the endosomal network and are released after they fuse with the plasma membrane. In contrast, microvesicles are formed through direct budding from the plasma membrane. Exosomes and microvesicles differ in size and are differentially enriched with various marker proteins. Overall, EVs can travel far distances and affect recipient cells after they are internalized through endocytosis or direct fusion with the recipient cell membrane. Figure adapted from [89]. 


\section{Extracellular vesicle cargo and uptake}

EVs contain an array of molecular cargo, including proteins, mRNA, DNA, and other nucleic acids bound by a lipid bilayer. Importantly, the composition of EV cargo is not derivative of the cytosolic content as proteins and RNAs are actively sorted into EVs [90-92]. However, changes to the cellular environment, such as oxygen tension, $\mathrm{pH}$ changes, cellular damage, or stress can increase the cellular production of EV or alter their cargo [93-95]. Cellular metabolism can also affect EV production and use of aerobic glycolysis is positively correlated with EV release [86]. Thus, the metabolic strategy of the conceptus may affect its EV production.

The major constituents of EV membranes include cholesterol, sphingomyelins, and PC phospholipids with significant enrichment of sphingomyelin, gangliosides, PS phospholipid, and di-saturated lipid populations [96, 97]. Lipid makeup can also vary across the different subtypes of EV. For example, PE phospholipid is enriched in microvesicles and not exosomes. The EV membrane lipids create a rigid bilayer and protect the EV cargo from extracellular proteases. EVs become more fluid and amenable to transfer of contents in acidic environments likely due to changes in membrane fluidity and composition [93]. Additionally, EVs may contain bioactive lipids such as leukotrienes, endocannabinoids, or lysophospholipids that can signal in recipient cells. EV can also account for transcellular transport of activatable phospholipases and prostaglandins $[98,99]$.

The nucleic acid cargo of EV is extremely diverse. In particular, EVs are enriched with small noncoding RNA such as mRNA, miRNA, tRNA, and piRNA. 
The mRNA molecules contained within EV can be translated into proteins by recipient cells [100]. Additionally, miRNA within EV can regulate gene transcription once internalized [101]. Various studies have reported the presence of DNA in EVs as well, and thus EVs may allow for the horizontal transfer of genetic information. Overall, increased cell proliferation, migration, and adhesion, which are important processes for conceptus elongation and embryo development, are common reported biological processes altered by the nucleic acid cargo of EV.

The protein cargo in EV has also been well characterized in many systems. The most common proteins are marker proteins such as major histocompatibility complex class II (MHC Class II), tetraspanins (CD9, CD81), endosomal sorting complex proteins (Alix, TSG101), and chaperone proteins (HSP70 and 90) [102]. Most of these markers are found on both exosomes and microvesicles. Other proteins found in EV are more dependent on the subtype of EV and the secreting cell, but can include enzymes (GAPDH, peroxidases, and pyruvate kinases), signal transduction proteins (G proteins, RAB11) and protein kinases [103]. Calculations suggest that individual vesicles may contain between hundreds to thousands of proteins depending on vesicle size [104].

Once in the extracellular space, EV must reach their target recipient cell. While EV can have autocrine effects the prevalence of this is not clear. The rigid lipid bilayer of EV protects EV from degradation and allows them to travel to distal locations for uptake by recipient cells $[105,106]$. There are three mechanisms for EV to elicit biological effects on recipient cells: 1) Activation of a cellular surface receptor; 2) Uptake via endocytosis of the vesicle; and 3) Uptake by fusion with 
the cell membrane [107]. Docking of EV is thought to be primarily mediated by surface proteins on the EV. Some EV contain ECM components, such as fibronectin and laminin, that promote binding, docking, and activation of surface receptors on recipient cells without EV uptake [108]. Recipient cell uptake of EVs is largely dependent on the protein cargo of EV and their own surface proteins. Almost all EV contain integrins and tetraspanins on their surface which may promote interaction with selective target cells $[109,110]$. Endocytosis of $E V$ is likely the major pathway of uptake and can occur through clathrin-mediated or clathrinindependent endocytosis, phagocytosis, or micropinocytosis [111, 112]. Although not every well characterized, endocytosis of EV likely targets them to the lysosomes and requires rescue by specific proteins that cause EV to fuse with MVB membranes to release their content into the cytosol [113]. Alternatively, if rescue of EV from lysosomal degradation does not occur, breakdown of EV cargo could be a relevant source of metabolites to the recipient cell. Evidence that EVs can deliver cargo by membrane fusion has been reported under acidic conditions and may be associated with the lipid composition of EVs [93]. Direct fusion with the plasma membrane would almost immediately release their cargo into the cytoplasm for use by the recipient cell.

Extracellular vesicles in the uterine lumen

Several studies have collectively indicated that EV are abundant in the female reproductive tract. EV are present in the follicular fluid and may mediate communication between different populations of granulosa cells. In cats, oviductal EVs fuse with the sperm cell acrosome and improve sperm motility and fertilizing 
capacity in vitro [114]. EVs are produced by cultured bovine oviductal epithelial cells in vitro and likely promote fertilization processes [115-117]. Thus, there is ample evidence that EVs play critical roles in several different female reproductive processes.

Critically, EVs are present within the uterine lumen of both cyclic and pregnant ewes and are internalized by both the conceptus and endometrium [118]. Uterine EVs from sheep are positive for two common EV markers (CD63 and HSP70) and contain endogenous Jaagsiekte sheep retrovirus (enJSRV) protein, IFNT, and PTGS2, among others as well as mRNA and miRNA molecules [119]. Immunostaining for proteins associated with EV release, including HSP70, suggest that the LE and GE are the primary sources of endometrial EV [120]. Cultured conceptuses secrete EVs into culture media and the presence of IFNT in uterine EVs suggest that the conceptus also contributes EVs to the ULF of pregnant animals. The effects of pregnancy on the makeup and production of uterine EV have not been fully explored and remain an important area to study. As EVs are present in the uterine lumen of many species during the peri-implantation period of pregnancy, they are likely critical mediators of TE and endometrial communication during early pregnancy (Figure 5) [119, 121-124]. 


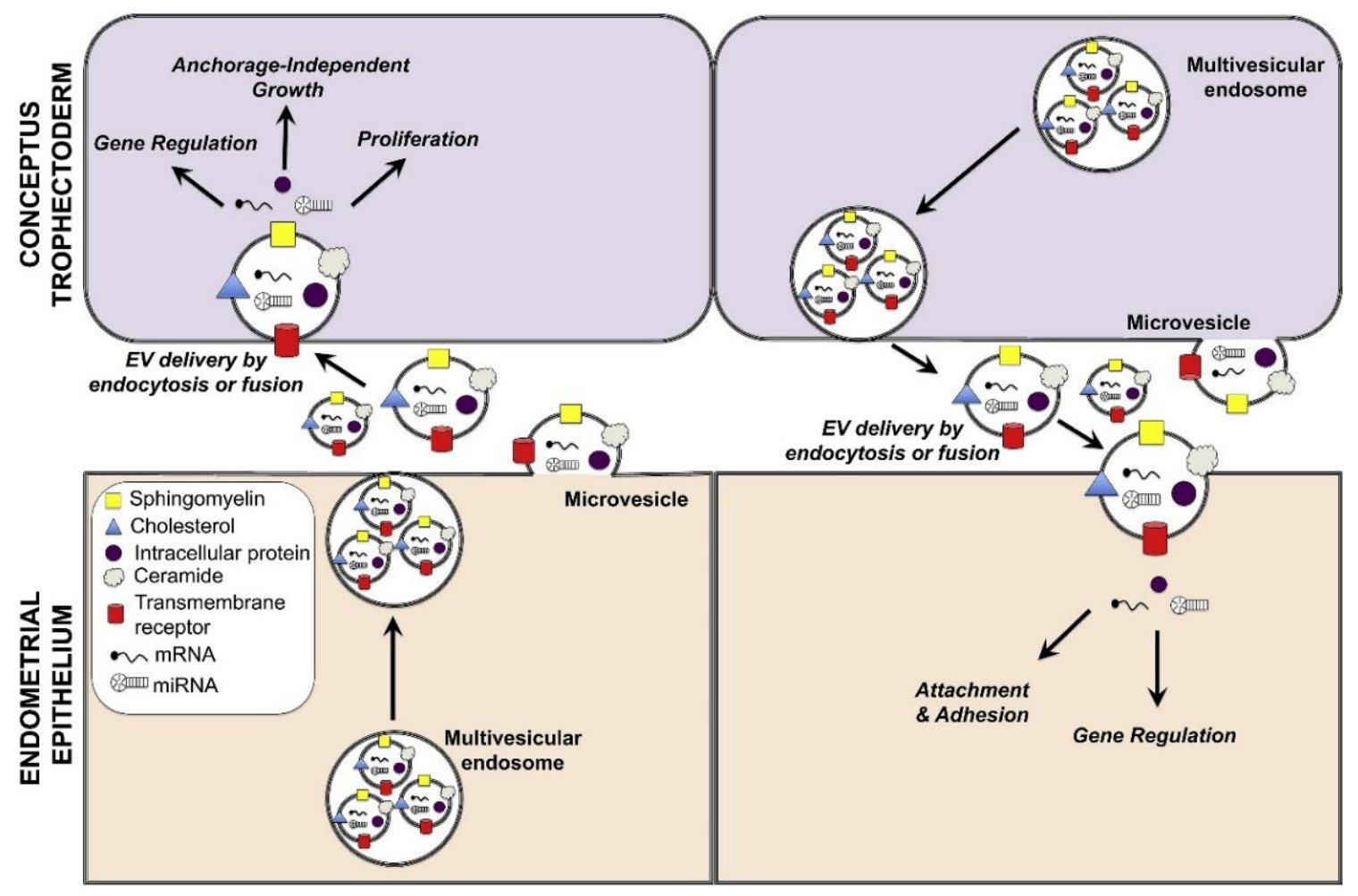

Figure 5. Working hypothesis on the role of extracellular vesicles (EV) in early pregnancy. Both the endometrium and the conceptus trophectoderm synthesize and secrete exosomes and microvesicles in the uterine lumen. Uptake of uterine EVs by endocytosis or membrane fusion can alter gene regulation, anchorage-independent growth, and proliferation in the conceptus and promote attachment and adhesion processes in the endometrium as well as gene expression. Figure from [125].

The cargo of uterine EVs is hormonally regulated and depends on the cell of origin, species, and pregnancy status. Alterations in EV cargo during the cycle could provide a mechanism to synchronize embryo development and the uterine endometrium [124]. In sheep, P4 increases the total number of EVs released and regulates the miRNA cargo of EV. The miRNA cargo increased by P4 were predicted to regulate adhesion and migration processes in the conceptus TE [126]. Bidirectional exchange of uterine EVs between the endometrium and TE occurs 
both in vitro and in vivo in sheep, cattle, mice, pigs, and humans [119, 121, 127, 128].

Various studies have demonstrated that EVs regulate embryo development at various developmental timepoints. EVs isolated from pregnant mice increase embryo cleavage and blastocyst development rates [129]. Further, embryoderived EVs have paracrine effects and transfer EVs between various embryonic subpopulations to promote cell migration [130]. Beyond early embryo development, EVs may play critical roles in mediating embryo growth and implantation processes. In sheep, EVs isolated from the ULF stimulate TE proliferation and IFNT secretion in vitro [120]. In humans, endometrial EVs transfer proteins that increase the cell adhesion and invasion capacity of an in vitro TE cell model $[131,132]$. Importantly, the protection of EV cargo may enhance their ability to regulate embryonic growth compared to other soluble proteins in the ULF, as exposure of an in vitro TE cell model to EVs increased TE adhesiveness and outgrowth compared to soluble proteins isolated from the ULF. Furthermore, EV exposure during early embryo development increased implantation rates in mice after embryo transfer compared to embryos exposed to soluble proteins alone [133]. Thus, EVs may have more important roles and promote TE proliferation, adhesion, and implantation processes.

Uterine EVs may also regulate the endometrium. Exposure of a bovine LE cell line to EVs from day 15 and 17 pregnant ULF increased the expression of classical ISGs including ISG15, MX1, MX2, STAT1, and STAT2 [123, 134]. In contrast, EVs isolated from day 20 and 22 ULF up-regulated the expression of 
adhesion molecules in an in vitro LE line, demonstrating differential effects of EVs by day [135]. In pigs, in vitro studies suggest EVs may promote endometrial angiogenesis [121]. Several studies have also sought to understand the effect of embryo derived EVs in modulation of the maternal immune system with conflicting results. For example, EVs downregulate inflammatory genes and reduce the ability of some endometrial cell lines to respond to an LPS challenge in vitro, suggesting an immunosuppressive role $[136,137]$. However, other studies have reported that EVs stimulate the NFKB signaling pathway and promote the production of IL-1 $1 \beta$ in other endometrial cell lines, suggesting a proinflammatory role [108, 134]. These inconsistencies may be due to differences in origin and/or population of EV, timing, species, cell line, or some alternative reason and are worth further study. To date, studies that investigate the effect of uterine EVs on the endometrium have not been conducted in sheep and have exclusively used in vitro systems that may not fully recapitulate EV effects on the endometrium in other animals.

\section{Metabolites and Lipids}

Metabolites are small molecular intermediates or products of cellular metabolism. As conceptus elongation is driven by cellular proliferation, the substrate demand for proteins, nucleic acids, carbohydrates, and lipids is great and likely affected by metabolite bioavailability. While glucose and amino acids in the ULF of cyclic and pregnant ewes has been well described, information on other metabolites is lacking. 


\section{Metabolites in the uterine lumen}

Glucose is present in the uterine lumen of sheep, cattle, mice, and humans. In sheep, glucose triples in the uterine lumen between days 13 and 16 of pregnancy and total glucose is higher than cyclic ewes on days 12 through 16 [138]. These increases correspond to the upregulation of several glucose transporters in the endometrial epithelia of pregnant ewes by both P4 and IFNT $[72,139]$. Although the endometrium of mice and humans does not appear to have a high gluconeogenic capacity, the rate limiting enzymes of gluconeogenesis, glucose-6-phosphatase (G6PC), phosphoenolpyruvate carboxykinase 2 (PCK2), and fructose-1,6-bisphosphatase 1 (FBP1), are expressed in the bovine uterus and are upregulated in the endometrium of day 17 pregnant heifers, a day which is roughly equivalent to day 14 in ewes [140]. Glycogen is also present in the ovine endometrium and is depleted between days 14 and 22 of pregnancy [141]. These findings may indicate that the ruminant uterus does not only transport glucose into the uterine lumen but also produces and stores it for use in pregnancy. While other saccharides have not been quantified in the ovine uterus, there is evidence that fructose and glucosamine are also present in the uterine lumen of sheep [142].

Amino acids likely play critical roles in early pregnancy events as well. All twenty proteinogenic amino acids are present in the uterine lumen of sheep, as well as citrulline, $\beta$-alanine, ornithine, and taurine. Most amino acids increase in abundance between days 3 and 14 of the estrous cycle, and all amino acids are higher in the uterine lumen of pregnant sheep by days 14 or 15 [138]. Glycine and serine are the most abundant amino acid in the ULF in both sheep and cattle, while 
arginine, glutamine, and leucine show the most marked increase in pregnancy. This large-scale increase in amino acid levels in the uterine lumen has been observed in other species including cattle, mice, and pigs [138, 143, 144]. Transportation of amino acids into the uterine lumen is likely mediated by increases in a few select neutral and acidic amino acid transporters in the uterine epithelial cells of pregnant ewes [140,145, 146]. Other amino acid transporters are temporally expressed by the endometrium during the estrous cycle and likely contribute to amino acid transportation by the LE.

Recent studies have characterized the presence of tricarboxylic acid (TCA) cycle intermediates in the bovine uterine lumen. Citrate, isocitrate, succinate, fumarate, malate, and alpha-ketoglutarate are all present in the ULF of cyclic cattle on days 12, 13 and 14 and alpha-ketoglutarate decreases between days 12 and 14 [147]. Proteomic analysis of the bovine ULF found that several of the enzymes that regulate the TCA cycle are also present in the uterine lumen [148]. In mice, a comparison of the ULF metabolome between days 1 and 4 (day 4 being the time of embryo implantation) found that fumarate and malate increased in the ULF while succinate and alpha-ketoglutarate decreased [143]. Although TCA cycle intermediates have not been described in the ovine ULF, various solute carriers for TCA intermediates are expressed in the ovine endometrium such as SLC13A5, a sodium-dependent citrate transporter whose expression increases in the LE between days 14 and 16 of pregnancy [72, 140,149]. In cattle, expression of two TCA cycle enzymes, aconitase 2 (ACO2) and the rate-limiting enzyme isocitrate dehydrogenase 1 (NADP+) (IDH1), are elevated by pregnancy on day 17 [140]. 
Whether increases in the TCA cycle of endometrial cells only serves to meet endometrial metabolic demands or also increases TCA metabolites in the uterine lumen is unclear and warrants further investigation.

Purine and pyrimidine metabolites are also present in the ULF of heifers on days 12 to 14 of the estrous cycle. Specifically, the four ribonucleosides (adenosine, cytidine, guanosine, and uridine) and several nucleobases (adenine, guanine, hypoxanthine, and xanthine) are detectable $[147,150]$. In pigs, metabolites of purine (i.e. xanthine and uric acid) are inversely correlated with conceptus elongation, possibly indicating that the conceptus incorporates nucleotides from the ULF into DNA and RNA and does not actively catabolize them [151]. Proteins that bind and transport nucleotides are present in the ovine ULF and may promote their transportation to the conceptus [73]. Overall, the demand for nucleic acid substrate to generate DNA, RNA, and metabolic cofactors such as $\mathrm{NADH}$ during conceptus elongation is likely large and is one area that has not been sufficiently studied.

Lipids in the uterine lumen

Lipids are a broad class of molecules classified into eight categories: fatty acids, glycerolipids [monoacylglycerols (MAG), diacylglycerols (DAG), and triacylglycerols (TAG)], phospholipids, sphingolipids, sterols, prenols, saccharolipids, and polyketides [152]. Due to the complexity of each class, only five of these (fatty acids, glycerolipids, phospholipids, sphingolipids, and sterols) will be discussed. Like proteins, P4 increases the total luminal lipid content (summing across all identified lipids in a comprehensive lipidome experiment) of 
cyclic heifers $[153,154]$. Phospholipids seem to be the lipid class most susceptible to P4 regulation as administration of exogenous P4 increases several individual phosphatidylcholine (PC) and phosphatidylethanolamine (PE) phospholipids, as well as choline, phosphoethanolamine, and glycerophosphoinositol, metabolites used in phospholipid biogenesis [153]. In sheep, lysophosphatidic acid (LPA), a phospholipid derivative with critical roles in cell signaling, accumulates in the uterine lumen of pregnant ewes between days 12 and 15 [155]. In cattle, five PC phospholipids are higher in the ULF of pregnant heifers compared to cyclic heifers on day 17 [156]. Phospholipids are critical components of cellular and organelle membranes and important hubs for cell signaling, and thus may be limiting components during conceptus elongation. Other lipids that were higher in the uterine lumen of pregnant heifers include three TAGs and two short-chained acylcarnitines [156]. While other studies have not characterized TAG levels within the ovine uterine lumen, carnitine and acylcarnitine are also higher in the ULF of day 14 and 16 pregnant ewes compared to day 12 cyclic ewes [75]. Acylcarnitines are FA chains with a carnitine moiety attached that allows them to be transported across the mitochondrial membrane for FA oxidation and may indicate that FA oxidation by the endometrium or conceptus is active during conceptus elongation. Palmitic acid (C16:0) and stearic acid (C18:0) are the predominant non-esterified FAs (NEFA) present in the uterine lumen of cattle. Most NEFAs do not vary between cyclic and pregnant heifers except for arachidonic acid (20:4) which is lower in pregnant heifers likely due to use for prostaglandin biosynthesis [156, 157]. It is not surprising that total NEFA are not different in the uterine lumen, as 
NEFA are not a primary mode of lipid transportation and elevated levels are toxic in other organ systems $[158,159]$. Other lipid classes such as sphingolipids and sterols in the uterine lumen are poorly described in both sheep and cattle, although one of two measured sphingolipids [N-palmitoyl-sphingadienine (d18:2/16:0)] is elevated by P4 in cyclic heifers and cholesterol shows temporal changes between days 12 and 14 of the estrous cycle [153]. Although it is unclear how lipids are transported in the ULF, various studies have suggested they may be bound in extracellular vesicles or lipoproteins $[157,160,161]$

The origin of lipids within the ULF (e.g. endometrium vs. serum) is unclear. Endometrial lipid droplets may be one source since they accumulate in the LE during the estrous cycle under regulation by $\mathrm{P} 4$ but are depleted in pregnant ewes by day $15[162,163]$. Lipid droplets predominantly store neutral lipids such as TAG and cholesteryl esters, so if these are mobilized into the uterine lumen it is unclear if their lipid cargo is transformed by the endometrium into the wide variety of lipid classes present or if they would be mobilized as they are. In the ovine LE, lipid metabolism is one of the most upregulated cellular processes between days 12 and 14 of pregnancy due to an increase in genes like acyl-coenzyme A synthetase short-chain family member 2 (ACSS2), a cytosolic enzyme that activates acetate for lipid synthesis, and serine palmitoyl transferase, long chain base subunit 1 (SPTLC1), the initial enzyme in sphingolipid biosynthesis [72]. In the bovine endometrium, fatty acid synthase (FASN), fatty acid desaturase 2 (FADS2), fatty acid elongase 1 (ELOVL1), and ELOVL6 are upregulated in the pregnant bovine 
endometrium on day 17 [140]. Taken together, endometrial lipid droplets and elevated lipid synthesis by the endometrium may be the origin of lipids in the ULF. Conceptus use of metabolites and lipids

The mass mobilization of uterine metabolites likely supplies the developing conceptus with substrate to support cellular proliferation and growth. The conceptus expresses various transporters for glucose (SLC2A1, SLC2A3, SLC2A4, SLC5A1, SLC5A11) and amino acids (SLC6A19, SLC7A5, SLC43A2, SLC1A4, SLC1A5, SLC6A19, SLC7A5, SLC7A6, SLC7A8, SLC43A2) [139, 145, 146]. Additionally, high expression of endocytic genes such as megalin (LRP2), phosphatidylinositol binding clathrin assembly protein (PICALM), clathrin A (CLTA), and CLTC suggest that conceptus may acquire substrate through endocytosis [72].

Many of the metabolites internalized by the conceptus are likely directed towards cellular metabolism by the conceptus TE. To support the rapid cellular proliferation seen in conceptus elongation, ovine conceptuses and other early mammalian embryos predominantly utilize aerobic glycolysis and convert glucose into lactate rather than catabolizing glucose into carbon dioxide by mitochondrial oxidative phosphorylation [164]. This strategy, called the Warburg effect, is utilized in cancer cells and occurs even if there is sufficient oxygen and nutrients in the environment $[165,166]$. Although the advantage of utilizing this inefficient strategy is still unclear, one of the prevailing theories is that the demand for sufficient carbon sources, biomass, and NADH, which is lost in oxidative phosphorylation, outweigh the need for ATP $[165,167]$. One notable feature of the Warburg effect is an 
increased reliance on amino acid metabolism. Glutamine is particularly important due to its ability to generate pyruvate or serve as a nitrogen donor for amino acid and nucleotide synthesis [168].

During early embryo cleavages and blastocyst development, the majority of glucose metabolized by the ovine embryo is converted to lactate with low levels of complete glucose oxidation [169]. Later during conceptus elongation, $90 \%$ of glucose utilized by the elongating conceptus is converted into lactate [170, 171]. The use of glycolysis and oxidative phosphorylation decrease during this period; however, the contribution of glycolysis to the overall estimated energy yield from glucose metabolism increases despite decreases in total ATP production. Approximately $3.5 \%$ of total glucose is used in the pentose phosphate pathway and accounts for about 15 to $20 \%$ of the total carbon dioxide produced by the conceptus [172]. These metabolic shifts are likely driven by hypoxic conditions as conceptuses cultured at $1 \%$ and $5 \%$ versus $20 \%$ oxygen levels decrease use of oxidative phosphorylation and increase lactate production [171]. This is likely physiologically relevant, as estimates of oxygen levels in the lumen of the uterus of various species ranges from 1.5 to $8.7 \% \mathrm{O}_{2}$ [173]. Glutamine is also actively catabolized by the ovine conceptus although rates of glutamine catabolism decrease in the presence of glucose [174].

While metabolites are likely utilized by the conceptus for metabolism, they can also activate mammalian target of rapamycin (MTOR) signaling pathways. Notably MTOR is a serine/threonine protein kinase that responds to changes in amino acid levels and energy and regulates many processes including cell 
proliferation, lipid, and nucleotide synthesis [175, 176]. Disruption of FK506binding protein 12-rapamycin-associated protein 1 (FRAP1), a key protein in MTOR signaling complexes, results in post-implantation embryonic lethality in mice due to stunted trophoblast outgrowth and failure of the ICM to proliferate [177]. In sheep, arginine, leucine, and glucose increase phosphorylation of the key MTOR complex proteins ribosomal protein S6 kinase beta-1 (RPS6K) and RACalpha serine/threonine protein kinase (AKT1) and increase cellular proliferation of ovine trophectoderm cells in vitro $[178,179]$. Leucine and arginine also regulate trophoblast motility in an MTOR- dependent manner [179-181]. Both glucose and fructose activate MTOR signaling and increase cell proliferation in porcine TE cell models [182]. Collectively, while amino acids and saccharides are likely actively metabolized by the conceptus, they also likely promote proliferation, adhesion, and migration of TE cells in an MTOR-dependent fashion during conceptus elongation. While most work has explored how amino acids and carbohydrates in the ULF may affect TE cells, TCA intermediates or metabolites of purine/pyrimidine synthesis are likely present in the uterine lumen of sheep and may also contribute to metabolism or proliferation of the conceptus. Metabolite turnover by the conceptus may also alter the ULF makeup [183].

The uptake and use of lipids by the ruminant conceptus has not been well investigated in contrast to other metabolites. Ovine conceptuses likely internalize lipids from the environment as they express lipoprotein receptors [e.g. lipolysis stimulated lipoprotein receptor (LSR) and low density lipoprotein receptor (LDLR)], lipid binding scavenger receptors [e.g. scavenger receptor class B, member 1 
(SCARB1)], and fatty acid transporters [e.g. fatty acid binding protein 5 (FABP5) and solute carrier family 27 fatty acid transporters (SLC27)]. Lipid metabolism is an enriched process during bovine conceptus elongation with changes in genes that regulate lipid uptake, lipid droplet formation, and biogenesis of phospholipids [157]. These gene expression changes likely affect conceptus use of lipids and promotes their growth during conceptus elongation. One of the few in vitro experiments of lipid roles in ovine TE models found that LPA stimulates phosphorylation of extracellular signal-regulated kinase (ERK) signaling proteins, increases cellular proliferation, and induces cytoskeletal changes of TE cells [155]. Currently, it is unclear what lipids the conceptus may use or metabolize, except for conceptus conversion of arachidonic acid into PG. Characterization of the lipids present in the ULF or conceptus could clarify what lipids are most essential or utilized by the TE during conceptus elongation.

\section{Prostaglandins}

\section{Biosynthesis of prostaglandins}

Prostaglandins (PG) are short-lived biologically active oxygenated fatty acids and are the best studied lipid molecule in early pregnancy. They are made in the cell after arachidonic acid (AA) is cleaved from cellular phospholipids by Phospholipase A2 and transformed by one of the prostaglandin-endoperoxide synthase (PTGS) enzymes [184, 185]. There are two major PTGS enzymes: PTGS1 and PTGS2. In most tissues, PTGS1 is constitutively active and produces 
basal levels of PG, while PTGS2 is induced, typically by cytokine stimulation of cisregulatory elements upstream of the PTGS2 gene. Both enzymes are encoded by distinct genes and share approximately $\sim 60 \%$ nucleotide sequence similarity [186188] . They are structurally homologous to each other, except for an 18 amino acid insertion in the PTGS2 membrane binding domain that may act as a signal domain to regulate its localization or degradation. Additionally, a three amino acid substitution in the PTGS2 cyclooxygenase active site makes its active site $20 \%$ larger than PTGS1 and this size discrimination accounts for PTGS2's susceptibility to nonsteroidal anti-inflammatory drugs (NSAID) [189, 190]. Both PTGS enzymes are integral membrane proteins that are typically located on the endoplasmic reticulum or the outer membrane of the nuclear envelope. They form homodimers for AA conversation which occurs across two distinct active sites. The first active site binds a heme group and catalyzes oxygenation of $A A$ to form the intermediate prostaglandin $\mathrm{G}_{2}$ (PGG2). In the second step, PGG2 enters a hydrophobic pocket in the PTGS enzyme and is reduced to prostaglandin $\mathrm{H}_{2}$ (PGH2) [191-193]. Enzymatic conversion of AA to PGH2 by either PTGS1 or PTGS2 is the ratelimiting step for PG biosynthesis and is followed by downstream conversion to PG by prostaglandin synthase enzymes (Figure 6). 

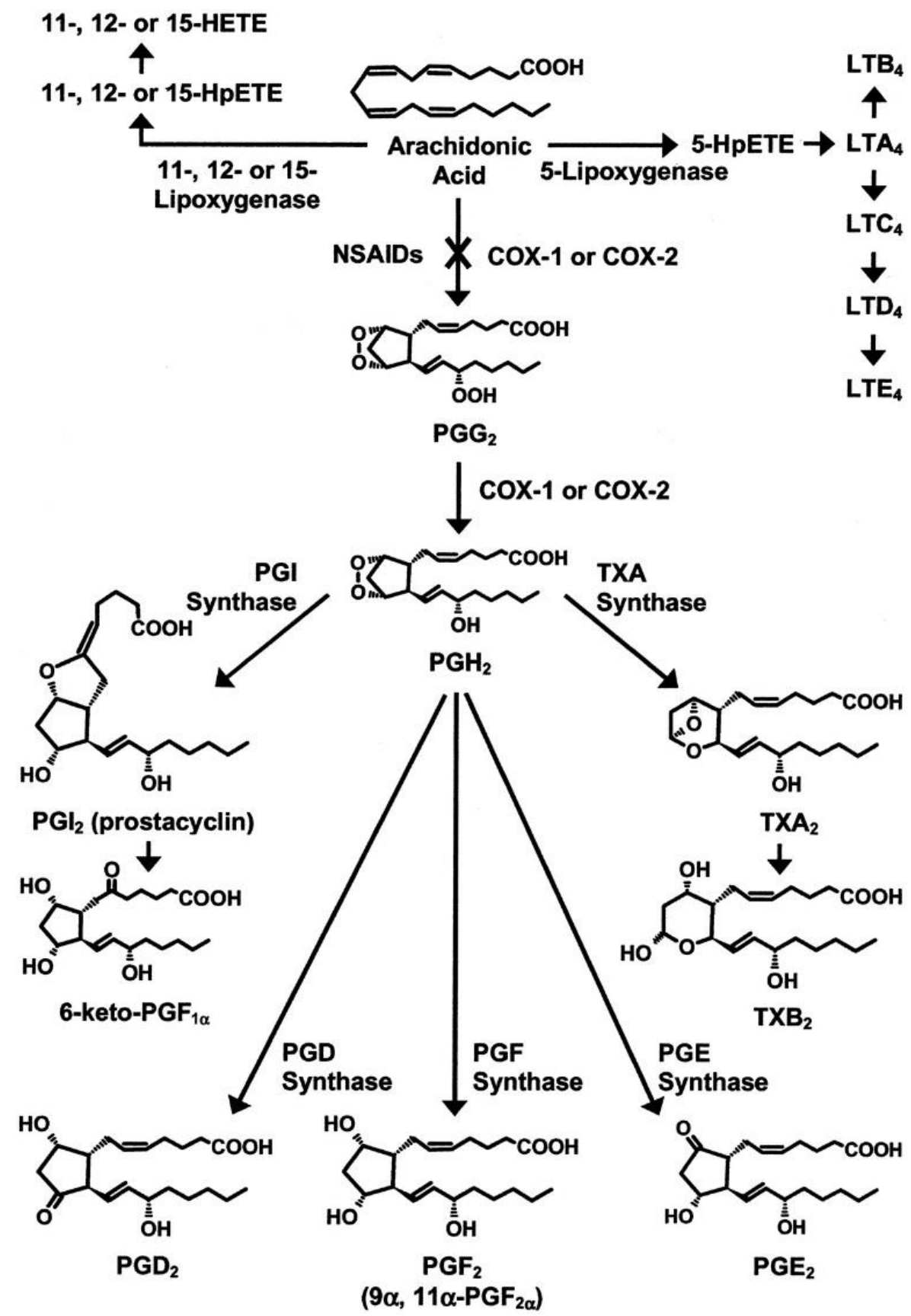

Figure 6. Schematic of prostaglandin (PG) biosynthesis. After arachidonic acid is cleaved from the phospholipid by phospholipase A2, arachidonic acid is converted in a two-step process into prostaglandin $\mathrm{H}_{2}$ (PGH2) by the prostaglandin-endoperoxide synthase (PTGS) enzymes 1 and 2 (PTGS1/PTGS2) enzymes (also referred to as cyclooxygenase COX 1 and 2). Downstream PG synthase enzymes can convert PGH2 to one of several different PG. Figure from [194]. 
The major PG are prostaglandin $\mathrm{E}_{2}$ (PGE2), prostacyclin (PGI2), prostaglandin $\mathrm{D}_{2}$ (PGD2), and prostaglandin $\mathrm{F}_{2 \alpha}(\mathrm{PGF} 2 \alpha)$. These are synthesized by their own dedicated PG synthase and isomerase enzymes and transported outside of the cell via transporter proteins called PG transporters (PGT) [195-197]. The physiological actions of PG are largely mediated by the PG receptors, a family of $G$ protein-coupled receptors $[198,199]$. The functions of $P G$ vary by tissue, receptor type availability, and receptor affinity. The downstream effects of PG binding depend on coupling of $P G$ receptors with G proteins. While most of the receptors typically associate with a single G protein, alternative splicing of the prostaglandin E receptor (PTGER) mRNA determines which G protein it associates with and which downstream actions PTGER activation will cause [200]. Additionally, each PG can stimulate most of the PG receptors with varying affinity allowing for differential downstream effects for each PG. Overall, PG activation of the classical PG receptors stimulates processes such as vasoconstriction or vasodilation, muscle contraction, and immune cell recruitment, among others [201, 202].

Prostaglandins can also regulate gene expression directly through peroxisome proliferator-activated receptors (PPAR). PPARs are nuclear receptors that bind many different ligands including PG, polyunsaturated FA (PUFA), and other related molecules [203-207]. Upon ligand binding, PPARs undergo conformational changes and form a heterodimer with retinoic $\mathrm{X}$ receptor (RXRs) [208]. These heterodimers act as transcription factors that bind to peroxisome proliferator hormone responsive elements (PPRE) in the promoter region of genes. 
The three primary PPARs are PPARA, PPARD, and PPARG. Each of these PPAR target alternative sets of genes and mediate different effects [209, 210].

\section{Prostaglandins in the uterine lumen}

The uterine lumen of cyclic and pregnant sheep, cattle, humans, and many other species contain substantial quantities of $P G$ from both the endometrium and embryo [211-213]. In sheep, PTGS1 is present in the LE, GE, and stromal cells of the endometrium throughout the estrous cycle, whereas PTGS2 protein appears specifically in the LE and superficial GE between days 10 and 12 of the ovine estrous cycle at levels higher than PTGS1 [50, 214]. Induction of PTGS2 is coincident with loss of PGR in the endometrial epithelial cells and causes an accumulation of $P G$ in the uterine lumen between days 10 and 14 of the estrous cycle [215]. The classical PG receptors PTGER2, PTGER4, and PTGFR are present in the LE and GE of the endometrium $[216,217]$. Pregnant ewes have more PG in their uterine lumen than cyclic ewes due to PG production by the conceptus and IFNT- stimulated increases in PG production by the underlying endometrium of pregnant ewes. This elevated capacity to produce PG by the endometrium is not regulated at the level of PTGS enzymes, as mRNA and protein are not more abundant. Instead, IFNT stimulates PGE2 synthase (PTGES) and PGF2 $\alpha$ synthetase (PTGFS) mRNA, leading to increases in PGE2, PGF2 $\alpha$, and 6ketoPGF1 $\alpha$ production by the endometrial epithelial cells in both sheep and cattle $[140,216,218,219]$.

Pregnant ewes have elevated amounts of $P G$ in the uterine lumen due to conceptus production of PG as well. The conceptus secretes greater amounts of 
PG per mg of wet tissue than the underlying endometrium between days 10-16. This production is regulated by PTGS2 as expression of PTGS1 is very low during early development while PTGS2 expression increases 16 -fold at the onset of elongation $[157,212,220]$. When cultured in vitro with radioactive $A A$, conceptuses converted AA to 6-keto PGF1a (41.4 $\pm 4.8 \mathrm{ng} / \mathrm{mg}$ tissue), PGF2 $\alpha(32.1 \pm 17.9$ ng/mg), PGE2 (12.3 $\pm 7.5 \mathrm{ng} / \mathrm{mg})$, and PGFM $(8.4 \pm 6.2 \mathrm{ng} / \mathrm{mg})$ [221]. Notably, PG are hypothesized to have autocrine, paracrine, and intracrine effects on the ovine conceptus. Ovine conceptuses express PGT and SLCO2A1, PG transporters, at levels greater than the underlying endometrium $[212,222]$. The $P G$ receptors PTGFR, PTGER2 and PTGER4, and PTGIR are also expressed by the ovine and bovine conceptus, and there is more than a 15-fold increase in expression of PPARG at the onset of elongation in ovine and bovine conceptuses $[72,140,157$, $161,212]$.

Results from recent studies demonstrate that PG may be key regulators of the endometrial environment during conceptus elongation in sheep. In one study, several blastocysts were transferred to cattle and were collected prior to conceptus elongation. Although IFNT was not yet detectable in the ULF, classical Type I ISG were already upregulated in the endometrium and it was hypothesized that PG, which were elevated in the uterine lumen, may have mediated these gene expression changes [223]. Expanding on this hypothesis, the intrauterine infusion of IFNT with meloxicam, a NSAID that targets PTGS2 specifically, decreased the ability of IFNT to stimulate ISG such RSAD2, GRP, and ISG15 in the endometrium and decreased the expression of $\mathrm{P} 4$ regulated genes including GRP, LGALS15, 
and CST3, indicating that PG may work synergistically with IFNT and P4 to alter gene expression in pregnant ewes [216]. Additionally, PG appear to have differential effects on gene expression in the endometrium. Incubation of bovine endometrial stromal cells with PGF2 $\alpha$, but not PGE2, increased HSD11B1 protein and total cortisol in culture media of stromal cells [224]. Further, infusion of individual PGE2, PGI2, and PGF2 $\alpha$ at the level produced by the conceptus found differing effects on gene expression in vivo compared to IFNT infusion [225]. For example, IFNT, PGE2, and PGI2 increased expression of EFNA1 and IGFBP1, while PGF2 $\alpha$ had no effect on it. Similarly, IFNT, PGF2 $\alpha$, and PGI2 stimulated CST3 expression while PGE2 had no effect. Infusion of individual PG also promoted the expression of glucose, fructose, and amino acid transporters in the uterine epithelia. Although PG infusion did not alter glucose levels in the uterine lumen, infusion of both PGE2 and PGF2 $\alpha$ increased total number of amino acids in the uterine lumen while infusion of IFNT and PGI2 did not. Almost all individual amino acids were elevated by the various PG apart from tyrosine and valine, which were reduced by IFNT and PGI2, and glutamine, which was decreased by IFNT, PGE2, and PGI2. Finally, infusion of only PGE2 or PGF2 $\alpha$ alone elevated the total recoverable amounts of amino acids while IFNT, PGF2 $\alpha$, and PGI2 did not [225]. Thus, PGs regulate elongation- and implantation- related genes in the endometrium and mobilize amino acids into the uterine lumen to promote conceptus growth and development.

Studies investigating PPAR binding in sheep and other species have also implicated important roles of PG in embryo survival, implantation, and placentation. 
In one loss-of-function study morpholino antisense oligonucleotides, which inhibit mRNA translation, were targeted to PPARG and infused into the uterine lumen of sheep. Conceptuses exposed to PPARG morpholinos were severely growth retarded on day 14 of pregnancy and had apoptotic trophectoderm while infusion of nonspecific control morpholinos or morpholinos targeted to PPARD had no effect [222]. In mice, PTGS2- derived PGI2 mediates embryo implantation via activation of PAPRD and PPARG inactivation in mice leads to embryonic lethality by day 10.5 due to underlying placental defects $[226,227]$. Taken together, PPAR likely bind PG and play critical autocrine roles in embryo development.

Most importantly, infusion of meloxicam into the uterine lumen of pregnant ewes between days 8 and 14 prevented production of PG by both the conceptus and endometrium and prevented elongation of conceptuses in sheep [216]. Similar experiments in pigs and mice have also found that pharmaceutical inhibition of PTGS2 alone or PTGS1 and PTGS2 results in pregnancy loss in these species [228, 229]. Interestingly, loss of PTGS2 in mice is not embryonic lethal in utero while mature PTGS2-null mice suffer infertility due to a myriad of pregnancy failures at the level of implantation and decidualization. Thus, endometrial PG are more important than embryonic PG production in early pregnancy in mice [229231]. Recent work in the pig has also demonstrated that conceptus-derived PG are not required for early conceptus development and can maintain pregnancy beyond day 30 [232]. Thus, while PG are undoubtedly critical regulators of conceptus elongation, the importance of conceptus production of PG in ruminant species is unclear. 


\section{Lipoproteins}

Lipoproteins are complex micelle particles used to transport hydrophobic lipids in aqueous environments. Although the lipid composition varies by lipoprotein class, all lipoproteins are composed of an outer monolayer of phospholipids, cholesterol, and apolipoproteins surrounding an inner hydrophobic core of TAG and cholesteryl esters (CE) [233-235]. This makeup exposes the hydrophilic head of phospholipids and apolipoproteins, making the overall particle water-soluble for transport in plasma and other extracellular fluids. Apolipoproteins play essential roles in the formation of lipoproteins as lipid stabilizers and the interface for receptor activation. The five major classes of lipoproteins include chylomicrons, very low-density lipoproteins (VLDL), intermediate-density lipoproteins (IDL), low-density lipoproteins (LDL), and high-density lipoproteins (HDL) (Figure 7) [233]. These classes are discriminated by differences in their floating density, their lipid makeup, their size, and their associated apolipoproteins. 

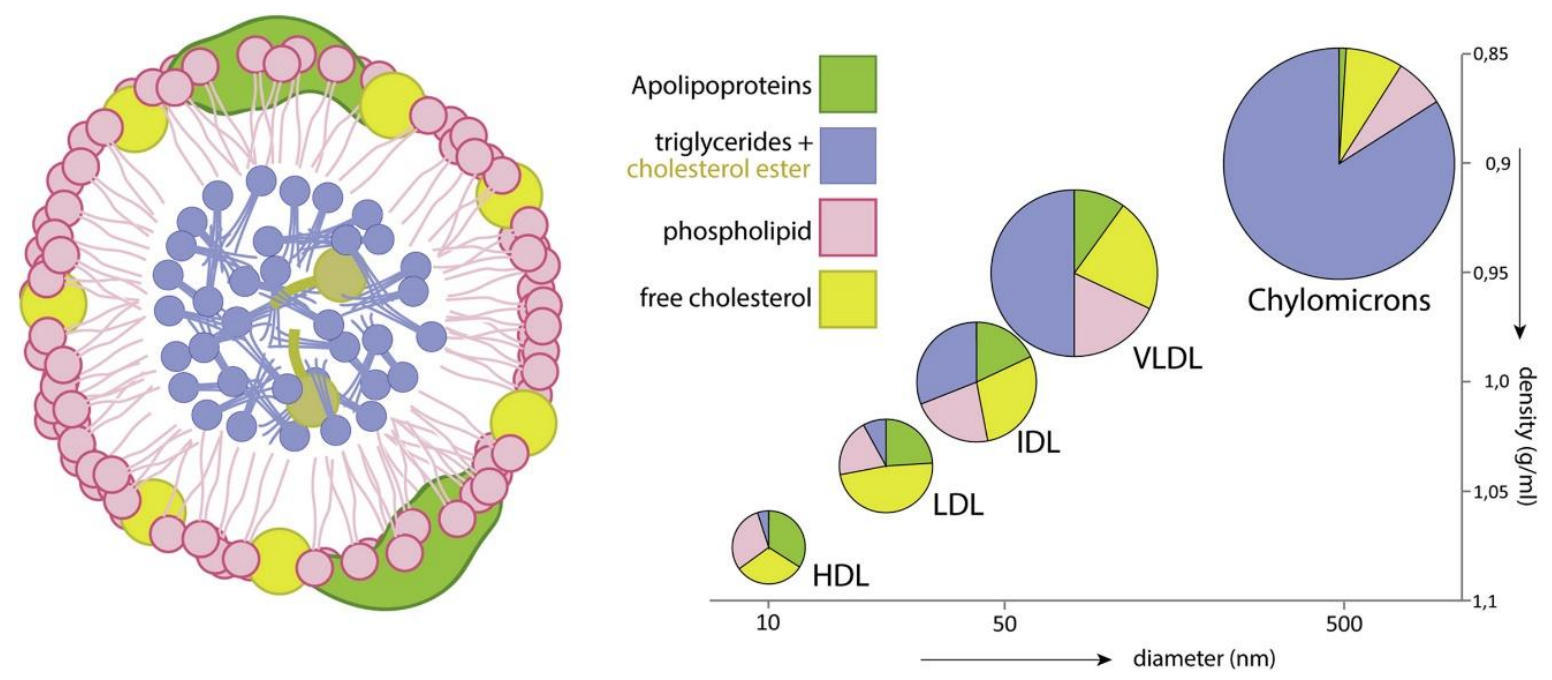

Figure 7. Lipoprotein classes. Lipoproteins are complex micelle particles that transport a core of hydrophobic lipids (e.g. triacylglyercides, cholesteryl ester) in aqueous environments. The five major classes of lipoproteins include chylomicrons, very low-density lipoproteins (VLDL), intermediate-density lipoproteins (IDL), low-density lipoproteins (LDL), and high-density lipoproteins (HDL). These classes are distinguished by their lipid and protein makeup, which affect particle diameter and density. Figure from [236].

\section{Biosynthesis of lipoproteins}

Chylomicrons are responsible for transporting digested dietary lipids from the intestine to the liver. Approximately $98 \%$ of chylomicrons is lipid and, of this, $95 \%$ is TAG, $<1 \%$ is cholesterol, and $5 \%$ is phospholipid [237, 238]. Chylomicrons are synthesized after uptake of dietary cholesterol, monoglycerides, and fatty acids from the intestinal lumen by the enterocyte [239-243]. These lipids are then repackaged and organized around apolipoprotein B-48 (APOB48), an isoform of the $A P O B$ gene that is specific to chylomicrons. After its synthesis, APOB48 it is taken to the endoplasmic reticulum lumen and fused with TAG-rich deposits by microsomal triglyceride transfer protein (MTTP), which is the rate-limiting step [244-246]. These premature chylomicrons are transported to the Golgi apparatus 
for secretion into the lymph and transportation to the circulation [247]. While in the circulation, chylomicrons acquire other apolipoproteins including APOC2 and APOE from other lipoproteins; apolipoprotein exchange in the circulation occurs due to their amphipathic nature and is a critical process for regulating lipoprotein maturity and clearance [248]. Chylomicrons deliver lipids to various tissues after lipoprotein lipase (LPL) hydrolyzes TAG into NEFA and MAG at the surface of capillary endothelium [249, 250]. Continuous hydrolysis of chylomicron TAG reduces it to a chylomicron remnant which are cleared after APOE binds to heparin sulfate proteoglycan and promotes its clearance by low density lipoprotein receptors (LDLR) [251].

The other TAG-rich lipoprotein, VLDL, is secreted by hepatocytes to deliver TAG from the liver to peripheral tissues. Like chylomicrons, VLDL are relatively lipid dense as $90 \%$ is lipid and only $10 \%$ is protein. Of the total VLDL lipid, approximately $55 \%$ is TAG, $15 \%$ is CE, and $20 \%$ is phospholipids [252]. The VLDL synthetic pathway is relatively similar to chylomicrons, although many of the lipids are from de novo synthesis and VLDL is bound to a different isoform of $A P O B$, APOB100 [253]. The rate-limiting step in VLDL synthesis is lipidation of APOB100 in the endoplasmic reticulum, and without sufficient lipids, APOB100 protein is degraded $[254,255]$. If enough lipids are available, VLDL is transported to the Golgi apparatus and secreted into the circulation. Nascent VLDL picks up APOC2, APOC3, and APOE from other lipoproteins to become a mature VLDL. The apolipoproteins APOE and APOC2 on the VLDL surface promote hydrolysis of lipids by LPL or hepatic lipase, while APOC3 inhibits it [256-258]. Continuous 
hydrolysis of TAG in VLDL reduces its size until it forms IDL, and eventually LDL. The exact lipid makeup of LDL can vary but overall it contains more cholesterol than TAG [259]. In humans, high levels of LDL in the circulation are associated with increased risk of atherosclerosis due to the high cholesterol content of LDL [260]. Clearance of both IDL and LDL is regulated by the total number of LDLR [261]. Endocytosis of IDL or LDL can be mediated by binding of APOE or specific recognition of the APOB100 protein. The number of LDLR on the hepatocyte surface is regulated by cholesterol content of the hepatocyte. Endoplasmic membrane bound proteins, called sterol regulatory element binding proteins (SREBPs), are transported to the Golgi apparatus if total cholesterol content of the endoplasmic membrane is low. Once in the Golgi apparatus, a soluble fragment in SREBP is proteolytically cleaved and enters the nucleus to upregulate $L D L R$ as well as other cholesterol regulating genes. When cholesterol levels are high, SREBP is retained to the ER, is not cleaved, and cannot upregulate $L D L R$ [262, 263].

In contrast to other lipoproteins, HDL regulates cholesterol and phospholipid efflux from peripheral tissues for clearance by the liver or kidney. The makeup of HDL is markedly different from other lipoproteins as only $60 \%$ of the mature HDL particle is lipid, while $40 \%$ is protein. The predominant lipid in HDL is phospholipids, which comprise $47 \%$ of the total lipid, and CEs, which account for $40 \%$ of total lipid [259]. The APOA1 protein is synthesized by hepatocytes and enterocytes and is secreted as a lipid poor protein into the circulation. Initial efflux of phospholipids and cholesterol by peripheral tissues is triggered by the 
membrane bound ATP-binding cassette transporter ABCA1 while ABCG1 is associated with lipid efflux to a more mature HDL particle [264-266]. The protein makeup of HDL particles includes many proteins that promote lipid efflux and binding to HDL. Lecithin:cholesterol acyltransferase (LCAT) is an enzyme that forms CEs for HDL transportation by esterifying PC phospholipid bound fatty acid to a cholesterol molecule [267]. Cholesteryl ester transfer protein (CETP) is a hydrophobic glycoprotein that promotes redistribution of lipids and apolipoproteins in HDL. Phospholipid transfer protein (PLTP) regulates the phospholipids in HDL and can incorporate lipid free APOA1 into an already mature HDL [268]. Additionally, HDL particles associate with proteins that regulate immune regulation and intravascular remodeling and can induce endothelial nitric oxide (NO) synthase (eNOS) expression and increase NO availability in endothelial cells [269, 270]. HDL is cleared from the blood after binding to scavenger receptor class $B$ member 1 (SCARB1) in the liver, kidneys, or other steroidogenic tissues [268, 271]. Uptake of whole HDL by SCARB1 occurs via caveolae-dependent endocytosis [270, 272]. In the kidney, LRP2 can endocytose HDL for clearance [273]. The interactions of lipoproteins with each other and with various tissues is shown in Figure 8. 


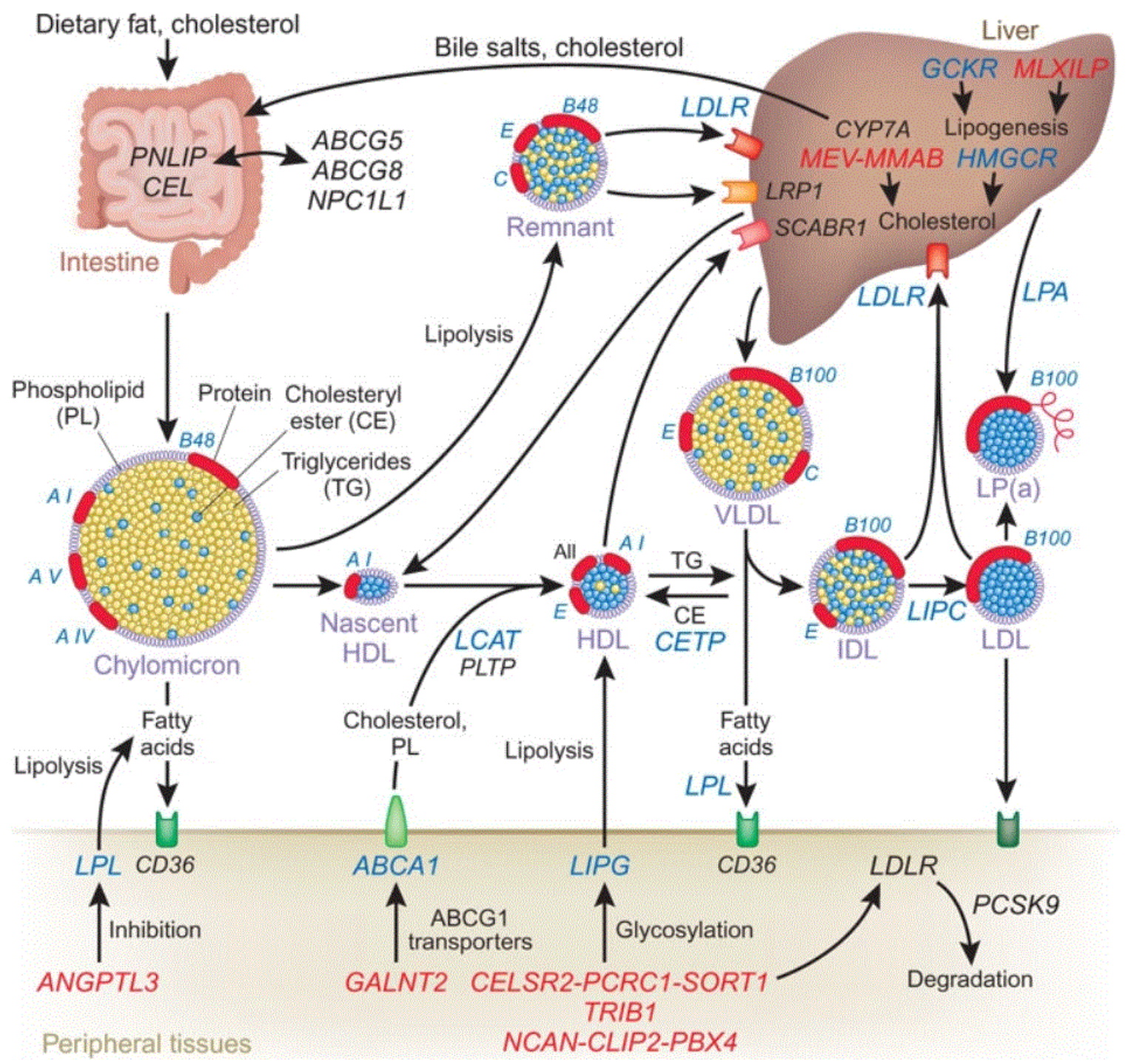

Figure 8. Lipoprotein interactions. The triglyceride-rich lipoproteins originate from the intestine and liver as chylomicrons and very low-density lipoprotein (VLDL), respectively. Both these lipoproteins deliver fatty acids to peripheral tissues after lipoprotein lipase (LPL) hydrolyzes bound triglycerides into fatty acids. After enough lipids are hydrolyzed, chylomicrons form chylomicron remnants and VLDL form intermediate-density lipoproteins (IDL) or low-density lipoproteins (LDL). These lipoproteins are then cleared by the LDL receptor (LDLR) on the liver. The high-density lipoproteins (HDL) are made after lipid-poor apolipoprotein A1 (APOA1) protein is secreted into the circulation. While in the circulation, APOA1 is lipidated with cholesterol and phospholipids from peripheral tissues after interaction with ATP-binding cassette transporter ABCA1. HDL is cleared from through scavenger receptor class B member 1 (SCARB1) receptors in the liver or kidney. While in the circulation, lipoproteins exchange apolipoproteins and lipid components. Figure from [274]. 
Importantly, most of the studies into the biosynthesis and makeup of lipoproteins come from studies using mouse models or studying genetic defects in humans. While ruminants utilize drastically different strategies for digestion, most of the described findings are thought to be conserved in ruminants. The major differences are in what lipoproteins are predominantly used. In cattle, VLDL are thought to be much scarcer in the plasma than they are in humans due to increased TAG synthesis by peripheral tissues. Additionally, evidence suggests that APOE protein is less abundant, possibly a reflection on decreased VLDL in the circulation. Importantly, HDL is the major plasma lipoprotein in ruminants [275, 276].

\section{Lipoproteins and apolipoproteins in the uterine lumen}

Lipoproteins are present within the female reproductive tract and play key roles in pregnancy establishment. For example, HDL regulate sperm capacitation by promoting lipid efflux and remodeling of the sperm head membrane in the oviduct $[277,278]$. Additionally, HDL supply cholesterol to granulosa cells for steroid hormone synthesis during the estrous cycle $[279,280]$. The presence of apolipoproteins in the uterine lumen indicates that lipoproteins are also present in the uterine lumen during early pregnancy, although their origin and function during conceptus elongation and implantation is not clear [157, 160, 161]. Many proteins involved in lipoprotein synthesis and uptake are expressed in the endometrium and show time, species, and pregnancy-dependent changes in expression. Endometrial expression of $A P O B$ is lower in pregnant heifers than cyclic heifers on day 17 while expression of $A P O A 1$ is not different [140]. In humans and primates, APOA1 is present in the endometrium and is downregulated by human chorionic 
gonadotropin ( $\mathrm{hCG}$ ) while APOE is highly expressed by the endometrium during the window for embryo implantation [281-283]. In mice, APOA1 is located on the apical side of the LE and production by the endometrium transiently increases at the time of implantation [284-286]. The bovine endometrium possibly internalizes lipoproteins since $V L D L R$ and $L D L R$ expression is higher in pregnant heifers than cyclic heifers on day 17 , while expression of SCARB1 is lower [140, 287]. Although there are not clear patterns across different species, there is enough evidence that the endometrium actively synthesizes apolipoproteins, and by inference lipoproteins, and expresses lipoprotein transporters.

During conceptus elongation, the ovine and bovine conceptus highly express $A P O A 1$ and, to a lesser extent, $A P O E[72,140]$. Embryonic APOA1 is secreted into the uterine lumen and is elevated in the uterine lumen of pregnant sheep and cattle at the time of conceptus elongation [73, 74, 288]. Interestingly, increased secretion of APOA1 is correlated with improved embryo quality in humans [289]. Several genetic studies support the hypothesis that apolipoproteins, and thus, lipoproteins, may play important roles in pregnancy. Inactivation of $A P O B$ in mice was embryonic lethal in mice by day 9 of gestation [290-292]. Infusion of APOA 1 targeting siRNA into the uterine lumen of mice resulted in fewer embryonic implantation sites and increased pregnancy loss $[285,286]$. In mice, inactivation of $A P O A 1$ itself is not embryonic lethal, but inactivation of the HDL interacting proteins $A B C A 1$ and $S R-B 1$ is due to placental malformation [293-295].

In summary, the embryo and endometrium both produce apolipoprotein, and by inference, lipoproteins, which accumulate in the ULF during pregnancy. 
However, the roles for lipoproteins in ruminant species is unclear. In sheep, lipoproteins may transport lipids to the conceptus during TE elongation and data in mice suggests that some lipoprotein components may play critical roles in embryo implantation or placentation. 


\title{
CHAPTER II:
}

\section{CHARACTERIZATION AND REGULATION OF EXTRACELLULAR VESICLES IN THE LUMEN OF THE OVINE UTERUS}

\author{
Eleanore V. O'Neil, Gregory W. Burns, Christina R. Ferreira, and \\ Thomas E. Spencer
}

This work is published in:

Biol Reprod. 2020 Apr 24;102(5):1020-1032. doi: 10.1093/biolre/ioaa019. PMID: 32055841. 


\begin{abstract}
Secretions of the endometrium are important for peri-implantation growth and development of the sheep conceptus. Extracellular vesicles (EVs) are present in the uterine lumen, emanate from both the endometrial epithelia of the uterus and trophectoderm of the conceptus, and hypothesized to mediate communication between those cell types during the establishment of pregnancy in sheep. Size exclusion chromatography and nanoparticle tracking analysis determined that total EV number in the uterine lumen increased from days 10 to 14 of the cycle but was lower on days 12 and 14 of pregnancy in sheep. Intrauterine infusions of IFNT did not affect total EV number in the uterine lumen. Quantitative mass spectrometric analyses defined proteins and lipids in EVs isolated from the uterine lumen of day 14 cyclic and pregnant sheep. In vitro analyses found that EVs decreased ovine trophectoderm cell proliferation and increased IFNT production without effects on gene expression as determined by RNA-seq. Collective results support the idea EVs impact conceptus growth during pregnancy establishment via effects on trophectoderm cell growth.
\end{abstract}




\section{Introduction}

In sheep, the embryo enters the uterus as a morula around day 6 (day $0=$ estrus), develops into a blastocyst that hatches from the zona pellucida by day 8 , and then grows into a spherical to ovoid shaped conceptus (embryo and associated extraembryonic membranes) by day $10[19,296]$. As the conceptus begins to elongate on day 12 , the trophectoderm produces substantial quantities of interferon tau (IFNT), the maternal recognition of pregnancy signal, that prevents production of luteolytic pulses of prostaglandin F2 alpha (PGF2 $\alpha$ ) by the endometrium, thereby maintaining the corpus luteum and progesterone (P4) secretion to establish pregnancy [11, 297, 298]. IFNT also modulates expression of genes in the endometrium that are likely important for uterine receptivity and conceptus growth $[299,300]$. By day 14 , the elongated conceptus is filamentous in nature, reaching $12-14 \mathrm{~cm}$ in length, and by day 16 the conceptus occupies the length of the uterine horn ipsilateral to the corpus luteum and begins to attach and firmly adhere to the uterine epithelium to initiate placentation for pregnancy establishment [296].

The endometrium of the uterus secretes factors into the lumen, collectively termed histotroph, that regulate growth and development of the conceptus [301]. Histotroph is essential for the elongation process, as in vitro and in vivo derived blastocysts fail to elongate unless transferred to a synchronized uterus $[64,65]$. Histotroph is mainly derived from the endometrial epithelia and is an undefined mixture of proteins, lipids, amino acids, sugars, ions, and other factors [154, 302304]. Altogether, these substances are proposed to mediate conceptus- 
endometrial interactions and promote conceptus development and pregnancy establishment. Indeed, uterine gland knockout ewes, which lack glandular epithelium (GE) and have reduced luminal epithelium (LE), experience recurrent pregnancy loss due to defects in conceptus elongation [66, 67, 305].

Exosomes and microvesicles, collectively termed extracellular vesicles (EVs), are cell-derived vesicles of 30 to $1000 \mathrm{~nm}$ in diameter that contain cargo, including select proteins, lipids, RNAs, and miRNAs [306, 307]. They are released into the extracellular space, where they can then be taken up by recipient cells and modulate recipient cell biology [307]. Recently, EVs were found to be a component of histotroph in the uterine lumen of several different mammals including humans and sheep $[119,124]$. In vitro analyses found that EVs are produced and released by ovine endometrial GE cells and human endometrial epithelial cells [124, 308]. In cyclic sheep, total EV number increased in the uterine lumen between days 10 and 12 post-estrus in response to progesterone [126, 309], which is coincident with the onset of conceptus elongation in pregnant sheep. Indeed, EVs from uterine lumen were shown to be internalized by both the conceptus trophectoderm and endometrial epithelium [118]. EVs purified from the lumen of the uterus of cyclic and pregnant sheep contain protein, mRNA, and miRNA cargo and modulate trophectoderm cell proliferation in vitro $[118-120,126,135]$. Thus, EVs are proposed to have a biological role in early conceptus-maternal interactions by impacting conceptus growth and development as well as implantation. The following studies were conducted to understand the regulation of EVs in uterus of 
pregnant ewes, identify lipids and proteins in them, and determine their effects on trophectoderm cell gene expression and proliferation.

\section{Materials and methods}

Animals

All experimental and surgical procedures for the mature sheep (Ovis aries) were approved by the Institutional Animal Care and Use Committee of the University of Missouri. Adult cycling ewes were checked daily for estrus (day 0) and euthanized on days 10,12 , and 14 of the estrous cycle ( $n=5-9$ per day) or days 12 and 14 of pregnancy after mating to an intact ram of proven fertility ( $\mathrm{n}=$ 5-8 per day). The entire female reproductive tract was obtained through a midventral incision, and the broad ligament removed with scissors. A clamp was placed on the internal ostium of the cervix, and the oviduct and uterotubal junction was removed. A Tomcat urinary catheter, attached to an all plastic syringe containing 10-ml sterile PBS ( $\mathrm{pH} 7.2$ ), was inserted about $1 \mathrm{~cm}$ into the uterine horn contralateral to the corpus luteum. Sterile PBS was slowly instilled into the uterine lumen and then gently massaged through the uterine body into and through the ipsilateral horn that was held over a sterile petri dish using a hemostat. The uterine lumen flush (ULF) was collected in a petri dish and the conceptus removed if present. The ULF was then transferred with a plastic pipette to a $15-\mathrm{ml}$ tube, clarified by centrifugation ( $3000 \times g$ for 15 min at $4^{\circ} \mathrm{C}$ ), transferred into a clean 15$\mathrm{ml}$ tube, and volume recorded prior to storage at $-80^{\circ} \mathrm{C}$. 
In another study, cyclic adult ewes were detected for estrus using a vasectomized ram. Using a surgical approach described previously [69, 216], day 10 post-estrus ewes were subjected to a midventral laparotomy, and the lumen of each uterine horn received a vinyl catheter (0007760; Durect Corp, Cupertino, California) connected to an Alzet 2ML1 osmotic pump (Durect Corp) secured by suturing the oviduct and mesovarium to the outside of the uterus. Ewes ( $n=5$ per treatment) received pumps containing the following: (1) $2 \mathrm{~mL}$ of saline vehicle as a control saline; or (2) $101 \mu \mathrm{g}$ recombinant ovine IFNT. The Alzet 2ML1 osmotic pump has a reservoir volume of $2 \mathrm{~mL}$ and a pumping rate of $10 \mu \mathrm{L} / \mathrm{h}$ for 7 days. Recombinant ovine IFNT was prepared as described previously [310]. The amount of recombinant ovine IFNT provided by the Alzet pump into the uterine lumen on a daily basis $(14.4 \mu \mathrm{g})$ mimics IFNT production by an elongating day 14 ovine conceptus, which is $600 \mathrm{ng}$ per $\mathrm{h}$ [311]. Previous studies found that infusion of 14.4 $\mu \mathrm{I}$ IFNT into the uterine lumen each day mimics effects of the conceptus on endometrial expression of hormone receptors and IFNT-stimulated genes during early pregnancy in ewes $[216,312]$. At necropsy on day 14 post-estrus, the female reproductive tract was excised, and ULF obtained as described in previously.

Extracellular vesicle isolation by size exclusion chromatography (SEC) and quantification by nanoparticle tracking analysis (NTA)

EVs were isolated and quantified from uterine flush using a previously described SEC method [126]. Clarified ULF was thawed on ice and filtered through a 0.22- $\mu \mathrm{m}$ PVDF syringe filter (cat \# SLGV033RS, EMD Millipore, Billerica, MA). Filtered ULF was concentrated by ultrafiltration with Centricon Plus-70 centrifugal 
filter units (cat \# UFC710008, EMD Millipore) at $2093 \times g$ for $15 \mathrm{~min}$, recovered at $1000 \times g$ for $2 \mathrm{~min}$, and brought to a volume of $500 \mu$ by weight with sterile PBS. EVs were isolated from the concentrated flush by SEC [313, 314] using qEVoriginal columns (Izon Science Ltd, Oxford, UK). Columns were equilibrated with $10 \mathrm{ml}$ of PBS and fractions 7 to $9(500 \mu \mathrm{l}$ each) were isolated, concentrated with an Amicon Ultra-4 device (cat \# UFC801008, EMD Millipore) at $2093 \times g$ for $15 \mathrm{~min}$, and brought to $200 \mu \mathrm{l}$ with sterile PBS. Aliquots of recovered EVs were diluted to approximately $10^{7}-10^{8}$ particles per $\mathrm{ml}$ with $5 \mathrm{mM}$ ethylenediaminetetraacetic acid (EDTA) in PBS and then evaluated by NTA using a NanoSight NS300 instrument (NanoSight Ltd, Amesbury, UK) equipped with a low-volume flow cell and syringe pump. The NanoSight was calibrated with 100 $\mathrm{nm}$ polystyrene beads (Polysciences, Warrington, $\mathrm{PA}$ ). Vesicle aggregates, initially observed as modal peaks in NTA, were disrupted by dilution of EVs with $5 \mathrm{mM}$ EDTA in PBS [315]. Videos were captured (camera level 13-14, syringe pump speed 30 , temperature control setting $25^{\circ} \mathrm{C}$ ) using the standard measurement protocol of five $60 \mathrm{sec}$ videos followed by processing with NTA software (NanoSight) to track each visible particle. The Stokes-Einstein equation was employed by the software to determine the size distribution and number of particles (concentration) within each sample.

Mass spectrometric analysis of proteins

Mass spectrometry (MS) analysis was conducted by the Gehrke Proteomics Center at the University of Missouri-Columbia. Briefly, EVs from day 14 cyclic or pregnant ewes ( $n=3$ per status) were lysed in Laemmli buffer (Sigma-Aldrich, St. 
Louis MO), and protein was precipitated with $100 \%$ acetone overnight at $-20^{\circ} \mathrm{C}$. Protein was digested with LysC directly in concentrated urea buffer for $3 \mathrm{~h}$ at $37^{\circ} \mathrm{C}$. Urea was diluted 6 -fold and protein digested with trypsin overnight at $37^{\circ} \mathrm{C}$. Next, peptides were purified and concentrated using Pierce C18 tips (ThermoFisher, Waltham, MA) according to the manufacturer's protocol. Peptides were lyophilized and resuspended in $80 \mu \mathrm{l}$ of $5 \%$ acetonitrile/ $0.1 \%$ formic acid solvent to approximately $1 \mu \mathrm{g} / \mu \mathrm{l}$ based on $\mathrm{C} 18$ tip binding capacity. Peptides were transferred to vials and placed in a refrigerated autosampler $\left(7^{\circ} \mathrm{C}\right)$. To analyze peptides by MS, a $1 \mu \mathrm{l}$ injection was made on a C8 trap column (ThermoFisher, $\mu$ precolumn - $300 \mu$ m i.d. x 5 mm, C8 Pepmap 100, $5 \mu \mathrm{m}, 100 \AA$ ) and separated on a $20 \mathrm{~cm}$ long $\times 75 \mu \mathrm{m}$ inner diameter pulled-needle analytical column packed with Waters BEH-C18 $1.7 \mu \mathrm{m}$ reversed phase resin. Peptides were separated and eluted from the analytical column with a gradient of acetonitrile at $300 \mathrm{~nL} / \mathrm{min}$. The Bruker nanoElute system is attached to a Bruker timsTOF-PRO mass spectrometer via a Bruker CaptiveSpray source. MS data were collected in positive-ion data-dependent PASEF mode over an $\mathrm{m} / \mathrm{z}$ range of 100 to 1700 .

The acquired data were submitted to the PEAKs $X$ search engine for protein identifications using a NCBI-Ovine-Bovine concatenated database. Data were searched with the following conditions: trypsin as enzyme and 2 missed cleavages allowed; carbamidomethyl cysteine as a fixed modification; oxidized methionine and deamidation of $\mathrm{N} / \mathrm{Q}$ as variable mods; and $50 \mathrm{ppm}$ mass tolerance on precursor ions, and 0.1 Da on fragment ions. Search results files were first filtered for $0.1 \%$ FDR (peptide false discovery rate) and 1 unique peptide per protein and 
export from PEAKS. The samples were grouped ( 3 cyclic and 3 pregnant), and data was further filtered for an average of 4 spectral counts for a group with spectral counts in 2 of 3 samples. A pair-wise comparison was done using PEAKSQ. This quantitation is based on precursor (peptide) intensity and is corrected for mass and retention time matching.

Lipid extraction and MS analysis by multiple reaction monitoring (MRM)-profiling

For MS analysis, EVs isolated from ULF of day 14 cyclic or pregnant ewes ( $n=3$ per status) were subjected to MS analysis by MRM-profiling at the Metabolite Profiling Facility of Purdue University. Lipids were extracted using the Bligh and Dyer method [316]. For that, $300 \mu \mathrm{L}$ suspension was transferred to a new microtube and mixed with $250 \mu \mathrm{L}$ of chloroform and $450 \mu \mathrm{L}$ of methanol. This solution was incubated at room temperature for $15 \mathrm{~min}$. After that, $250 \mu \mathrm{L}$ of chloroform and $250 \mu \mathrm{L}$ of water were added and the sample was centrifuged for 10 min at $16,000 \times \mathrm{g}$, forming a 2-phase solution where the bottom phase contained the lipids (organic phase). The organic phase was transferred to a new tube and dried using a Speedvac centrifuge (Savant Speedvac, Thermo Scientific Inc., San Jose, CA, US), and samples were stored at $-80^{\circ} \mathrm{C}$ until MS analysis.

Lipid profiling was performed using the MRM-profiling method similar to that recently described [317]. Dried lipid extracts were diluted in $50 \mu \mathrm{L}$ of methanol/chloroform $3: 1 \quad(\mathrm{v} / \mathrm{v})$ and $250 \mu \mathrm{L}$ of injection solvent (acetonitrile/methanol/ammonium acetate $300 \mathrm{mM} \mathrm{3:6.65:0.35(v/v))} \mathrm{to} \mathrm{obtain} \mathrm{a}$ stock solution. Final dilution of the stock solution was performed to obtain $10 \mathrm{e} 6$ ion signal for the PC/SM lipids, which are the most abundant lipid classes. Mass 
spectrometry data was acquired by flow-injection (no chromatographic separation) from $12 \mu \mathrm{L}$ of diluted lipid extract delivered using a autosampler to the ESI source of an Agilent 6460 triple quadrupole mass spectrometer (Agilent Technologies, Santa Clara, CA, USA). A binary pump (Agilent 1200) was connected to the autosampler and operated at a flow rate of $20 \mu \mathrm{L} / \mathrm{min}$ and pressure of $30 \mathrm{bar}$. Capillary voltage on the instrument was $3.5-5 \mathrm{kV}$ and the gas flow $5.1 \mathrm{~L} / \mathrm{min}$ at $300^{\circ} \mathrm{C}$.

Ovine trophectoderm (oTr) cell proliferation assays

Primary oTr cells, isolated from Day 14 elongated ovine conceptuses and characterized by our laboratory $[318,319]$, were used for in vitro studies of proliferation using previously described methods [319-321]. The oTr cells were maintained in exosome-free trophoblast growth medium [DMEM/F-12 supplemented with exosome-free 10\% Exo-FBS (System Biosciences), glutamine (2 mM), insulin $(700 \mathrm{nM})$, pyruvate $(1 \mathrm{mM})$, nonessential amino acids $(0.1 \mathrm{mM})$, and antibiotics (50 $U$ penicillin, $50 \mu \mathrm{g}$ streptomycin)] and maintained in a humidified $5 \% \mathrm{CO}_{2}$ environment at $37^{\circ} \mathrm{C}$.

For proliferation assays, $\mathrm{OTr}$ cells were seeded into 96-well plates and maintained in serum- and insulin-free DMEM/F12 for $24 \mathrm{~h}$. After $24 \mathrm{~h}$, treatments were added to each well ( $n=3$ wells per treatment) as follows: (1) DMEM/F12 medium alone; (2) trophoblast growth medium with $10 \%$ serum and $700 \mathrm{nM}$ insulin as a positive control; (3) increasing amounts of inert control vesicles (synthetic unilamellar $100 \mathrm{~nm}$ liposomes at $0.001,0.01,0.1$ or $1 \times 10^{10}$ particles per $\mathrm{ml}$ ); (4) increasing amounts of ULF EVs from Day 14 cyclic sheep $(0.001,0.01,0.1$ or 
$1 \times 10^{10}$ particles per $\mathrm{ml}$ ); or (5) increasing amounts of ULF EVs from Day 14 pregnant sheep $\left(0.001,0.01,0.1\right.$ or $1 \times 10^{10}$ particles per $\left.\mathrm{ml}\right)$. After 24,48 or $72 \mathrm{~h}$ of treatment, cell numbers were determined using an MTT cell proliferation assay (Promega, Madison, WI). The entire study was repeated at least three times with different passages of oTr cells and different ULF EV isolates.

Ovine trophectoderm gene expression assays

The oTr cells were seeded into 24 well plates and treated as described above for cell proliferation assays using liposomes or EVs from day 14 cyclic or pregnant ULF once they were $70-80 \%$ confluent. After 24 or $72 \mathrm{~h}$ of treatment, media was aspirated. For two replicates, media was frozen at $-20^{\circ} \mathrm{C}$ for Western dot blot quantification of IFNT. The oTr cells were rinsed in PBS, lysed in TRIzol reagent (ThermoFisher) for 15 minutes, scraped, and snap frozen in liquid nitrogen.

Total RNA isolation and $q P C R$ analysis

RNA was extracted from oTr cells using a chloroform-ethanol protocol. Samples were digested with DNAse I and then purified using a Direct-zol RNA column (Zymo Research, Irvine, CA). Total RNA (500 ng) was reverse transcribed using the iScript RT supermix (Bio-Rad, Hercules, CA). Reverse transcription was performed following manufacturer's instructions. For qPCR anlaysis, control reactions were run in parallel without reverse transcriptase to test for genomic contamination. Real time PCR was done in triplicate on a CFX384 Touch Real Time System with SsoAdvanced Universal SyberGreen Green Supermix (BioRad). PCR conditions were: activation, $95^{\circ} \mathrm{C}$ for $2 \mathrm{~min} ; 40$ cycles of $95^{\circ} \mathrm{C}$ for $5 \mathrm{sec}$; 
$60^{\circ} \mathrm{C}$ for $30 \mathrm{sec}$; and $72^{\circ} \mathrm{C}$ for $30 \mathrm{sec}$. Previously published primers for prostaglandin-endoperoxide synthase 2 (PTGS2) and glyceraldehyde 3phosphate dehydrogenase (GAPDH) were used (Dorniak et al. 2012). Primers for ovine IFNT were designed and synthesized by Integrated DNA Technologies (primer forward 5' CATCTCGACTCTGACGATTTCC 3', and reverse 5' CCATTGTGACCGTGAAGAAGTA3'). The $\triangle \mathrm{CT}$ was the difference between the cycle threshold (CT) for the reference gene GAPDH and the gene of interest. For visualization fold change was calculated relative to the reference genes $\left(2^{-\Delta \Delta C T}\right)$. Gene expression was analyzed by ANOVA using the GLM procedure of SAS. RNA Sequencing Data Analysis

Total RNA was isolated from cells as described above with a RNeasy MinElute cleanup kit (Qiagen, Valencia, CA). Eluted RNA was stored at $-80^{\circ} \mathrm{C}$ in nuclease-free water. Quality and concentration of RNA were determined using a Fragment Analyzer (Advanced Analytical Technologies, Inc., Ankeny, IA). Libraries were prepared by the University of Missouri DNA Core Facility using an Illumina TruSeq mRNA kit and sequenced on a NextSeq 500 instrument (Illumina Inc., San Diego, CA) to an average depth of 67 million reads (75 bp paired-end) per sample. Reads were adapter trimmed and quality trimmed to a sliding window quality score of 30 and minimum length of 20 bp with fqtrim software (version 0.9.4, doi:10.5281/zenodo.20552). Trimmed reads were mapped to the Ovis aries genome assembly (Oar_v3.1) using HISAT2 (version 2.0.3) [322]. Reads overlapping Ensembl annotations (release 84) [323] were quantified with featureCounts (version 1.5.0) [324] prior to model-based differential expression 
(DE) analysis using the edgeR-robust method [325]. Genes without evidence of expression (counts per million, CPM rowSum $<1$ ) were filtered out and quality control plots were generated. Transcriptome-wide read distributions were plotted for each sample with boxplots of log-transformed CPM values. A scatterplot of the first two principal components was constructed with the plotMDS function of edgeR to verify treatment separation prior to statistical testing. The false discovery rate (FDR) was set at 0.05 for DE analysis with edgeR-robust.

\section{Quantification of IFNT by Western dot blot analysis}

The amount of IFNT in the culture media was determined by a semiquantitative western dot blot analysis using a rabbit antibody specific for ovine IFNT and methods described previously (Brooks et al., 2015). Protein content of media was quantified by Qubit protein assay (ThermoFisher). Culture media protein $(20 \mu \mathrm{g})$ was loaded onto a nitrocellulose membrane in triplicate using a dot blot apparatus (Biorad) followed by rinsing with TBS. Membranes were blocked in $5 \%$ milk in $0.1 \%$ TBST (1x Tris-Buffered saline containing $0.1 \%$ Tween-20) and then incubated in IFNT antibody (1:1000) in blocking buffer overnight. Membranes were rinsed and then incubated in a goat-anti rabbit HRP conjugated secondary antibody at a 1:10,000 dilution. Samples were rinsed in 0.1\% TBST and then developed in SuperSignal West Pico Chemiluminescent Substrate (Thermo Scientific) for 5 minutes prior to visualization with a ChemiDoc MP System and Image Lab 4.1 Software (BioRad). ImageJ software V 1.6 (National Institute of Health, Bethesda, MD) was used to quantify the relative amount of IFNT protein. 
The data are adjusted for the recovered volume of cell culture media and expressed as total relative units of IFNT.

Statistical analysis

For the lipid profiles, the MRMs not presenting ion intensities above $30 \%$ compared to a blank sample (solvent injection) for at least one of the experimental samples were considered noise and removed from the statistical analysis. Next, the ion signal obtained for each MRM was divided by the total ion signal of the sample in order to obtain the relative ion abundances which were imported for statistical analysis at the MetaboAnalyst software (www.metaboanalyst.ca). First, most informative lipids were selected by receiver operating characteristics (ROC) curve analysis. Lipids from all presenting AUC of above 0.8 were used for principal component analysis (PCA) analysis. For PG lipids, selection using ROC AUC above 0.8 was not possible since only two lipids met this threshold. Therefore, only for this class, all lipids were included in the PCA. Data was normalized by autoscaling for PCA and for plotting heatmaps with cluster analysis.

All quantitative data were subjected to least-squares ANOVA using the general linear models (GLM) procedures of the Statistical Analysis System (SAS Institute Inc, Cary, North Carolina). In all analyses, error terms used in the tests of significance were identified according to the expectation of the mean squares for error. Significance $(P<0.10)$ was determined by the probability differences of the least squares means (LSM). 


\section{Results}

Effects of the estrous cycle, pregnancy and IFNT on extracellular vesicles in the lumen of the ovine uterus

The uterine lumen of cyclic and pregnant sheep ( $n=5-9$ per status) was gently flushed to recover the contents, and EVs in the ULF were purified by SEC and quantified by NTA. As illustrated in Figure 1, total EV number in the ULF was greater $(P<0.05$, day $x$ pregnancy status $)$ in cyclic than pregnant ewes. The mode size of the isolated EVs $(136.5 \pm 5 \mathrm{~nm})$ was not different $(P>0.10)$ in the ULF of cyclic or pregnant sheep.

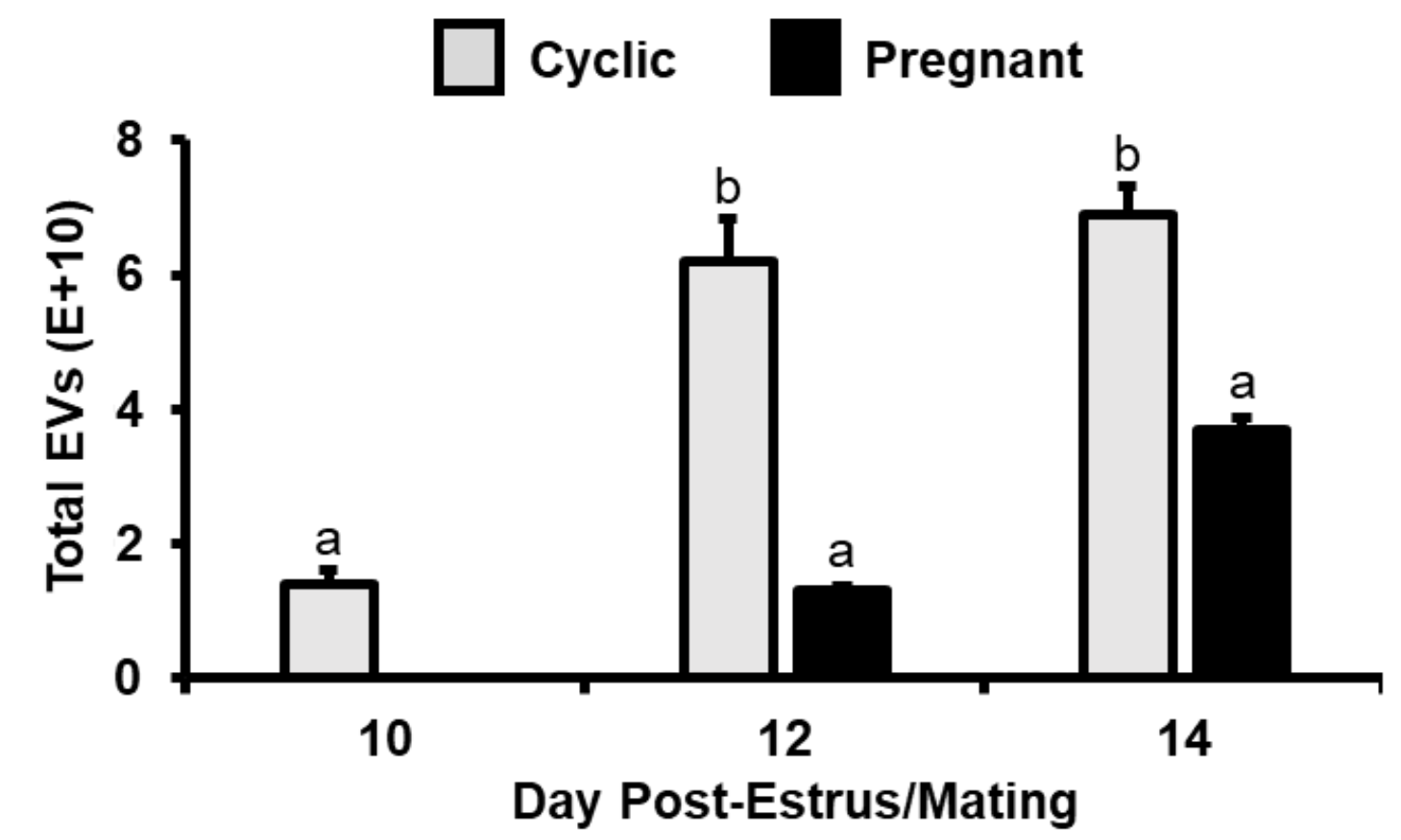

Figure 1. Extracellular vesicle (EV) number in the uterine lumen of day 14 cyclic and pregnant sheep. Different subscripts denote statistical significance $(P<0.05)$. 
A dominant factor secreted by the trophectoderm of the elongating ovine conceptus is IFNT. To test the hypothesis that IFNT from the elongating conceptus decreases EV production by the endometrium, vehicle as a control or recombinant ovine IFNT was infused into the uterine lumen of cyclic sheep between days 10 and 14 post-estrus using an osmotic pump. Total EV number (control vs IFNT: $1.1 \mathrm{E}+11 \pm 3.8 \mathrm{E}+10$ vs $1.59+\mathrm{E} 11 \pm 2.31 \mathrm{E}+10$ particles) and mode $\mathrm{EV}$ size (Control vs IFNT: $141.6 \pm 7$ vs $136.6 \pm 2 \mathrm{~nm})$ were not different $(P>0.10)$ in the ULF of ewes who received infusions of control vehicle as compared to IFNT.

Proteome of EVs in the ULF of day 14 cyclic and pregnant sheep

Uterine EVs were isolated from day 14 cyclic and pregnant ewes $(n=3$ ewes per status) and analyzed by MS. Analysis of uterine EVs identified 1,669 proteins (0.1\% FDR, 1 peptide minimum) in the samples with 126 and 289 unique to EVs from cyclic and pregnant ewes, respectively (Figure 2A; Supplementary Dataset S1 Tables 1A-C). Key exosomal protein markers (Alix, TSG101, CD63, HSP70, CD9, and CD81) were identified in all EV samples. The most abundant proteins identified in the EVs from both cyclic and pregnant sheep were alkaline phosphatase (ALPL), gag (Gag), an uncharacterized protein (LOC105602254), mucin 16 (MUC16), and lactadherin or milk fat globule-EGF factor 8 (MFGE8) (Table 1). The cyclic and pregnant samples clustered separately based on their protein profiles (Figure 2B). A total of 68 proteins that were present in EVs from both cyclic and pregnant EVs displayed a greater than two-fold change in abundance (Supplementary Dataset S1 Table 1D). Of these, 51 were higher in 
pregnant ewes, and 17 were higher in cyclic ewes. The top 10 most abundant proteins that were present in all samples and differentially abundant between cyclic and pregnant EVs are summarized in Table 2. To understand the potential functional impacts of proteins enriched in cyclic or pregnant EVs, gene names of the differentially abundant proteins identified were entered into a Generic Gene Ontology (GO) Term Finder [326], and significant GO terms were analyzed and visualized by REViGo [327]. Notably, proteins that were more abundant in pregnant EVs were related to protein translation, localization, and secretion (Figure 2C). Proteins more abundant in cyclic EVs matched only to one GO term that was negative regulation of wound healing.

Table 1. Ten most abundant proteins in EVs from the uterine lumen of sheep.

\begin{tabular}{cccc}
\hline \multirow{2}{*}{ Symbol } & Name & \multicolumn{2}{c}{ Spectral Counts (Mean \pm SE) } \\
\cline { 3 - 4 } & & Cyclic & Pregnant \\
\hline ALPL & Alkaline phosphatase tissue-nonspecific isozyme & $2173.3 \pm 111.6$ & $1863 \pm 419.6$ \\
Gag & Gag protein & $1744.0 \pm 616.6$ & $1285 \pm 792.4$ \\
& Uncharacterized protein LOC105602254 & $869.7 \pm 248.1$ & $589 \pm 370.1$ \\
MUC16 & Mucin-16 & $631.3 \pm 129.7$ & $626.6 \pm 181.1$ \\
ACTG2 & Cytoplasmic actin 2 & $612 \pm 157$ & $626 \pm 183$ \\
MFGE8 & Milk fat globule-EGF factor 8 protein & $798.3 \pm 228.5$ & $447.0 \pm 162.1$ \\
Env & Envelope Glycoprotein & $505.3 \pm 201.7$ & $391.6 \pm 148.9$ \\
LGALS3BP & Galectin-3-binding protein & $384.7 \pm 57.2$ & $492.7 \pm 78.6$ \\
ANPEP & Aminopeptidase N & $421.0 \pm 57.3$ & $435.6 \pm 52.4$ \\
EZR & Ezrin & $465.3 \pm 105.8$ & $335.0 \pm 73.3$ \\
\hline
\end{tabular}


Table 2. Top 10 differentially abundant proteins in EVs isolated from the uterine lumen of day 14 cyclic and pregnant sheep.

\begin{tabular}{ccccc}
\hline \multirow{2}{*}{ Symbol } & Name & \multicolumn{2}{c}{ Spectral Counts (Mean \pm SE) } & Fold \\
\cline { 3 - 5 } & & Cyclic & Pregnant & Difference \\
\hline SDCBP & Syntenin & $446.3 \pm 89.1$ & $238 \pm 97.4$ & 1.90 \\
CD109 & CD109 antigen & $229.3 \pm 68.2$ & $66.3 \pm 45.3$ & 3.45 \\
DYNCH1 & Cytoplasmic Dynein 1 Heavy Chain 1 & $80.3 \pm 29.3$ & $157.3 \pm 31.6$ & 0.50 \\
C3 & Complement C3 & $64.0 \pm 14.1$ & $129.7 \pm 63.6$ & 0.49 \\
MVP & Major vault protein & $41.3 \pm 2.4$ & $111.0 \pm 17.7$ & 0.37 \\
MUC5AC & Mucin 5AC & $36.6 \pm 10.2$ & $94.3 \pm 53.1$ & 0.38 \\
CPNE8 & Copine 8 & $85.0 \pm 22.3$ & $35.3 \pm 8.29$ & 2.40 \\
CFTR & Cystic fibrosis transmembrane & $31.7 \pm 5.5$ & $64.3 \pm 26.8$ & 0.49 \\
H3 & conductance regulator & $25.3 \pm 3.5$ & $63.0 \pm 17.6$ & 2.48 \\
PP13 & Histone H3 & $60.7 \pm 23.7$ & $24.7 \pm 8.9$ & 2.45 \\
\hline
\end{tabular}


A

B
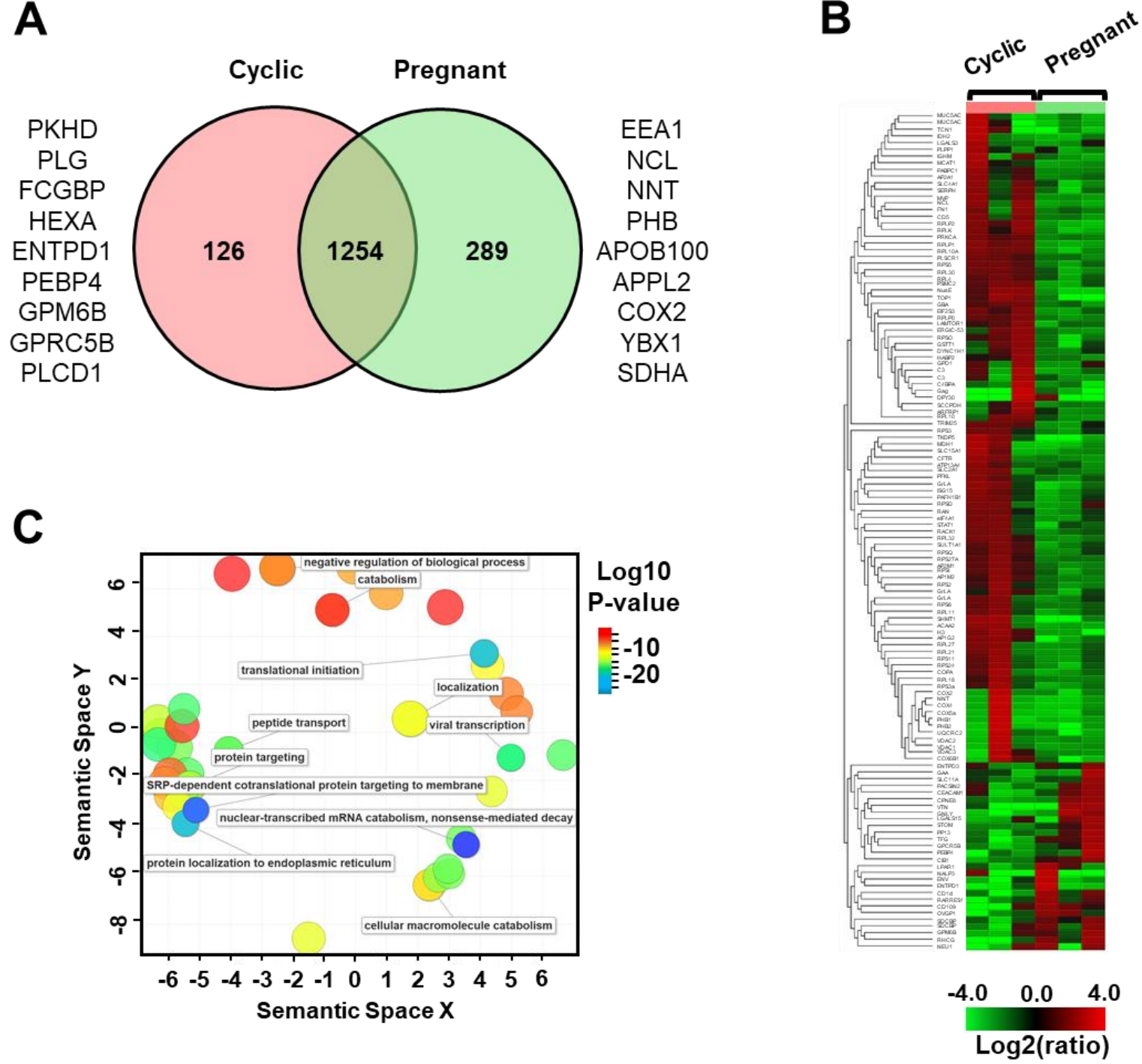

Figure 2. Proteomic analysis of extracellular vesicles (EV) isolated from the uterine lumen of day 14 cyclic and pregnant sheep. (A) Venn diagrams illustrating that of the total 1,666 proteins identified by mass spectrometry analysis, 126 and 289 proteins were unique to EVs isolated from cyclic and pregnant sheep, respectively. The gene names for the most abundant unique proteins identified in each pregnancy class are listed. (B) Heatmap clustering of proteins identified in EVs by mass spectrometry analysis. (C) Gene ontology (GO) analysis of proteins higher in EVs from ULF of day 14 pregnant sheep visualized using REViGO software. The scatterplot places gene ontology terms in a two-dimension plot to cluster processes with semantic similarities and removes functionally redundant GO terms. Color of circle indicates the FDR P-Value of the gene ontology term and size of circle indicates the frequency of the GO term in the underlying annotation database. 
Lipidome of ULF EVs from day 14 cyclic and pregnant ewes

ULF EVs were isolated from day 14 cyclic and pregnant sheep ( $n=3$ ewes per status) and subjected to comprehensive lipid profiling. Relative lipid amounts were compared across ten lipid subclasses based on the LipidMaps database. Eight classes of lipids were identified in ULF EVs from cyclic and pregnant ewes. There was no ion signal higher in EVs than for a blank sample was obtained for cholesteryl esters and triacylglycerols. The values of the various EV lipid populations (relative lipid amount as a percentage) are listed in Supplementary Dataset S2.

The ULF EVs contained phosphatidylcholines (PC), sphingomyelins (SM), $\begin{array}{llll}\text { phosphatidylethanolamines } & (\mathrm{PE}), \quad \text { phosphatidylglycerols } & (\mathrm{PGL}) \text {, }\end{array}$ phosphatidylinositols (PI), and phosphatidylserines (PS). PGL populations were very similar between EVs isolated from cyclic and pregnant ULF. In order to understand patterns across EV lipids, lipidomic data was denoised by removing lipids that had an AUC value below $80 \%$ after running them through classical univariate ROC curve analyses. The most relevant phospholipids showed some discrimination by pregnancy status. Of note, the 25 most relevant lipids (as indicated by t-test) in cyclic and pregnant ULF EVs grouped together across the phospholipid classes, indicating that there are patterns in phospholipid class that change between EVs in cyclic and pregnant ewes (Figure 3). 

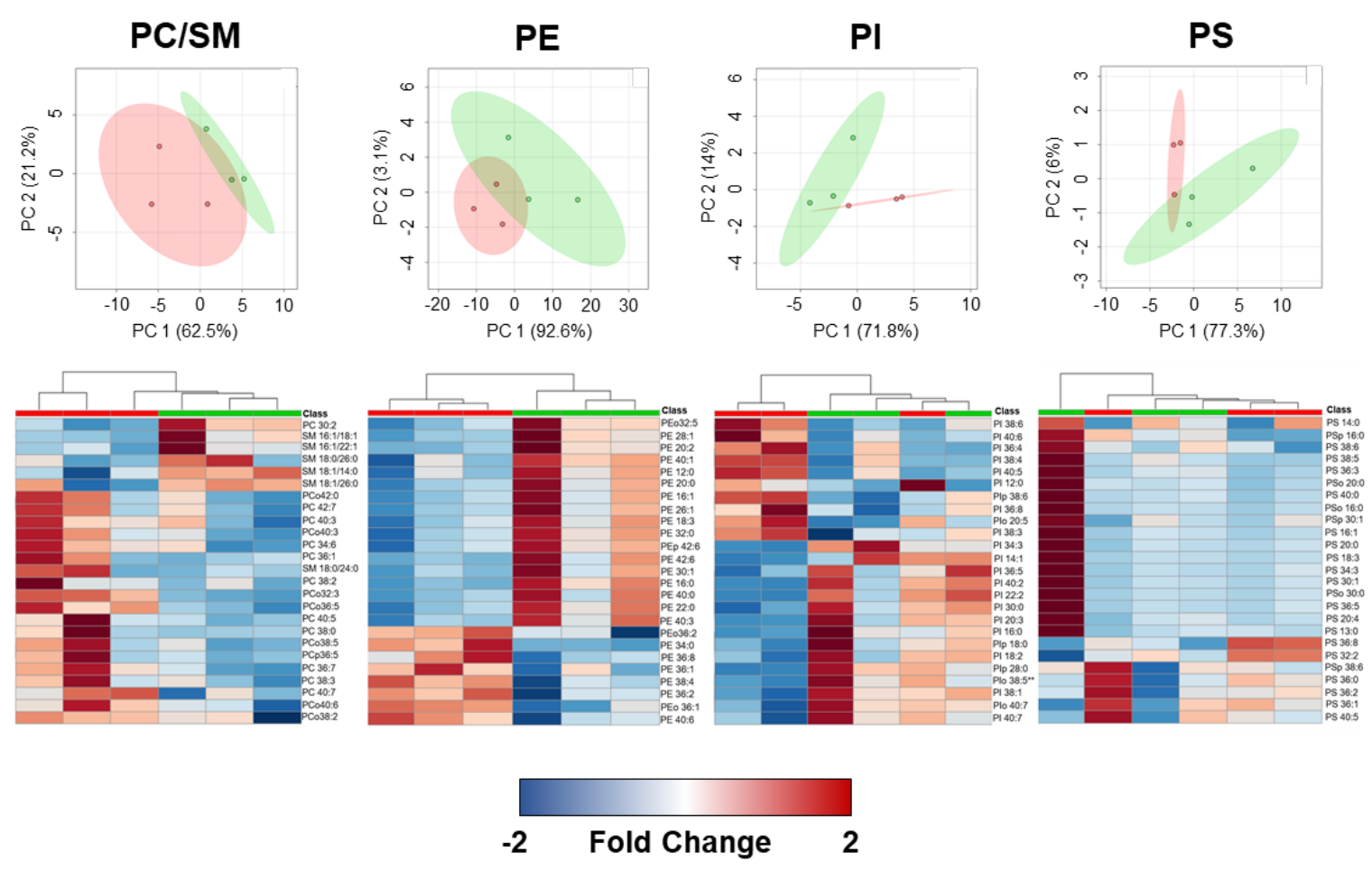

Figure 3. Phospholipids in extracellular vesicles (EV) isolated from the uterine lumen of day $\mathbf{1 4}$ cyclic and pregnant sheep. Phosphatidylcholine (PC) and sphingomyelin (SM), phosphatidylethanolamine (PE), phosphatidylinositol $(\mathrm{PI})$, and phosphatidylserine (PS) lipid profiles are shown. Principal component analysis (PCA) was used to visualize the difference in the lipid profiles and included only lipids presenting $A U C>0.8$ by ROC analysis (except for the PGL class). Heatmaps were generated using the top 25 most informative lipids of each class.

Ceramide, acylcarnitines, and FFA profiles are depicted in Figure 4. Ceramides displayed some separation by PCA analysis and clustered together by heatmap analysis. Acylcarnitines did not separate by PCA analysis or cluster together by heatmap analysis. EVs did not separate by pregnancy status in the FFA PCA analysis but clustered by heatmap analysis, with a noticeable shift in pregnant EVs away from palmitic (16:0) and stearic acid (18:0). Volcano plots and 
t-tests across several of the lipid classes identified lipids that were significantly different between EVs from cyclic and pregnant ewes (Supplementary Dataset S3).

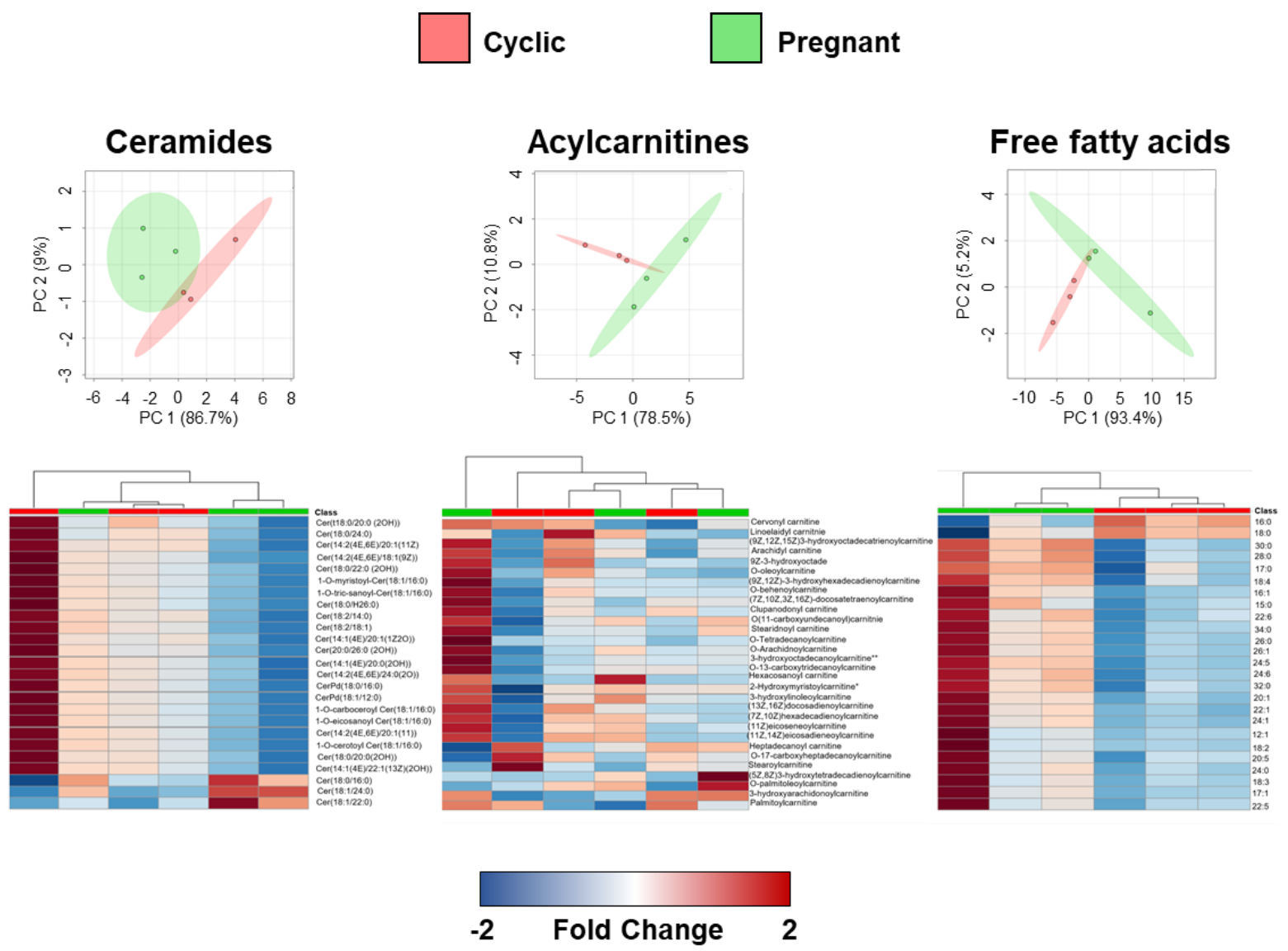

Figure 4. Ceramides, acylcarnitines, and free fatty acids (FFAs) in extracellular vesicles (EV) isolated from the uterine lumen of day 14 cyclic and pregnant sheep. Ceramide, acylcarnitine, and free fatty acid (FFA) lipid profiles are shown. Principal component analysis (PCA) was used to visualize the difference in the lipid profiles and included only lipids presenting AUC $>0.8$ by ROC analysis. Heatmaps were generated by the top 25 most informative lipid types of each class. 


\section{Effects of ULF EVs on oTr cell proliferation}

The effect of ULF EVs from day 14 cyclic and pregnant ewes was determined by in vitro analysis of oTr cell proliferation (Figure 5A). As a positive control, synchronized oTr cells were treated with 10\% FBS and insulin that substantially increased $(P<0.01)$ increased their proliferation at 48 and $72 \mathrm{~h}$ posttreatment compared to cells treated with DMEM/F12 media alone (negative control) (Figure 5B). Treatment of oTr cells with liposomes alone did not affect (P $>0.10)$ oTr cell proliferation. In contrast, treatment of oTr cells with the highest concentration of ULF EVs $\left(1 \times 10^{10}\right.$ particles per $\left.\mathrm{ml}\right)$ decreased $(\mathrm{P}<0.05)$ oTr cell proliferation at $72 \mathrm{~h}$ post-treatment when compared to oTr cells cultured with lower concentration of ULF EVs as well as DMEM/F12 alone (Figure 5B). This effect on oTr cell proliferation was observed with EVs isolated from ULF of both cyclic and pregnant sheep.

Effects of ULF EVs on oTr gene expression in vitro

oTr cells were serum starved for $24 \mathrm{~h}$ and then treated with ULF EVs from cyclic and pregnant ewes for $48 \mathrm{~h}$ at the high concentration ( $1 \times 10^{10}$ particles per $\mathrm{ml}$ ) or nothing as a control. Total RNA was harvested from the oTr cells and global gene expression assessed using RNA-Seq. EdgeR-robust analysis of the RNASeq data did not detect (FDR $\mathrm{P}<0.05)$ any effects of $E V$ treatment on gene expression in the oTr cells (Supplementary Dataset S4).

Next, oTr cells were serum starved for $24 \mathrm{~h}$ and then treated with ULF EVs at different concentrations or inert liposomes as a control for $24 \mathrm{~h}$ (Figure 6A). Real-time qPCR analyses found no effect of treatment on PTGS2 or IFNT mRNA 
levels, regardless of the concentration (Supplemental Figure S1). Media was collected to determine IFNT production by the oTr cells in response to treatment by Western dot blot analysis. Interestingly, the amount of IFNT in the culture media was increased $(\mathrm{P}<0.05)$ by treatment of oTr cells with either cyclic or pregnant ULF EVs at the high concentration $\left(1 \times 10^{10}\right.$ particles per $\left.\mathrm{ml}\right)$ for $24 \mathrm{~h}$, whereas inert control liposomes had no effect (Figure 6B).

A


Figure 5. Effect of extracellular vesicles (EV) isolated from the uterine lumen of day 14 cyclic and pregnant sheep on ovine trophectoderm (oTr) cell proliferation. (A) Experimental design. After a $24 \mathrm{~h}$ of serum starvation, oTr cells were treated with increasing concentrations of inert liposomes or ULF EVs. (B) Proliferation of oTr cells was measured at 24,48 , and $72 \mathrm{~h}$ post treatment using a MTT assay. Different subscripts denote statistical significance $(P<0.05)$. 


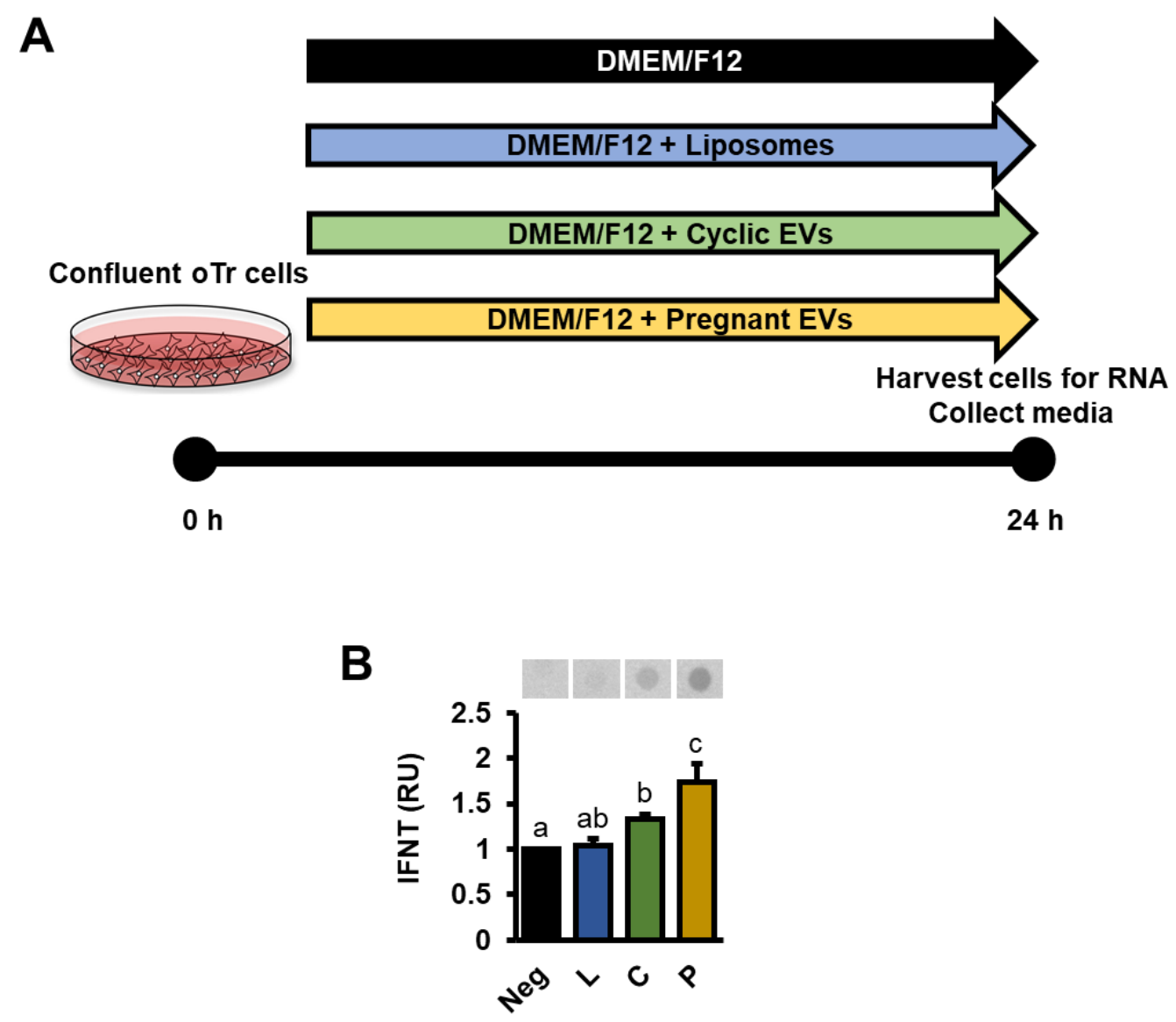

Figure 6. Effect of extracellular vesicles (EV) isolated from the uterine lumen of day 14 cyclic and pregnant sheep on IFNT production by ovine trophectoderm (oTr) cells. (A) Experimental design. Confluent oTr cells were treated with increasing concentrations of insert liposomes or ULF EVs. (B) IFNT was increased in culture media of oTr cells treated with the highest concentration ( $1 \times 10^{10}$ particles per $\mathrm{ml}$ ) of EVs using a Western dot blot analysis. Representative dots are shown above the graph. Different subscripts denote statistical significance $(\mathrm{P}<0.05)$.

\section{Discussion}

Available studies here and elsewhere support the idea that EVs have a biological role in conceptus-endometrial interactions during early pregnancy in sheep. Release of EVs into the uterine lumen increases after day 10 in cyclic ewes in response to progesterone [126]. In the present study, we found that the uterine 
lumen has fewer EVs on days 12 and 14 of pregnancy compared to the same days of the estrous cycle. In other cells, IFN-stimulated gene 15 (ISG15)-dependent ISGylation of proteins reroutes exosome-containing multivesicular bodies for lysosomal degradation, thus inhibiting exosome secretion [81]. In sheep, ISG15 is an IFNT-stimulated gene that is substantially up-regulated in the endometrial GE and stroma after day 11 of pregnancy by the elongating conceptus [302, 328]. Endometrial ISG15 actively conjugates to target proteins during early pregnancy [59]. In order to determine if the lower number of EVs in the pregnant uterine lumen is due to IFNT-dependent inhibition of endometrial EV release, IFNT was infused into the uterine lumen of cyclic ewes, and its effects on EV numbers was determined. Infusion of IFNT at equivalent levels to those secreted at the level of an elongating day 14 conceptus into the uterine lumen of cyclic ewes from days 10 to 14 post-estrus did not affect EV number or size in the uterine lumen. These results indicate that IFNT does not appear to regulate EV release by the endometrium. Thus, the lower EV number in early pregnant ewes is likely due to the uptake and utilization of EVs by the growing and elongating conceptus trophectoderm. Indeed, uptake of EVs by the ovine conceptus was demonstrated in vivo by visualization of fluorescently labeled EVs within and, importantly, in the membranes of trophoblast cells [118].

Here, the protein and lipid cargo of EVs isolated from ULF of day 14 cyclic and pregnant ewes was determined using MS analysis. The proteome of EVs isolated from ULF of day 14 cyclic and pregnant ewes has previously been described by our laboratory [119]. However, EVs were isolated from ULF in that 
study by ExoQuick-TC precipitation, while the present study used SEC. A recent comparison of EV isolation methods reported that EVs isolated by SEC result in higher EV recovery and significantly less protein contamination than EVs isolated by precipitation [313, 329]. Although there was overlap of proteins in the EVs reported in the present and our previous study [119], such as MX dynamin like GTPase 1 (MX1) and cathepsin L (CTSL), there were some key differences. The most abundant EV proteins reported in our previous study included serum albumin (ALB), which was not identified in the present study, and hemoglobin (HBM), which had fewer spectral counts in the present study. Both ALB and HBM are two of the most abundant proteins identified in ULF of sheep and cattle [72, 73, 75, 148], suggesting that the EV proteome previously described by our laboratory using EVs purified by ExoQuick-TC precipitation was contaminated by serum proteins that are quite abundant in the uterine lumen. There were also other differences in the EV proteome between the present and previous study. For example, prostaglandin-endoperoxide synthase 2 (PTGS2) was identified in EVs from the ULF of both day 14 cyclic and pregnant sheep, while our previous study identified PTGS2 in EVs only from day 14 pregnant ewes. The MS analysis in our present study identified many more proteins in the ULF EVs (1,000 proteins) than our previous study (195 proteins), presumably due to the use of a more sensitive MS approach.

The MS analysis of EVs in the present study identified many proteins in the EVs that are encoded by genes expressed by both the endometrial epithelia and the conceptus trophectoderm [72]. Indeed, several of the most abundant proteins 
identified are attributed to the endogenous Jaagsiekte sheep retroviruses (enJSRVs), including Gag, Pro-pol, and Envelope, which are very abundantly expressed in the endometrial LE and GE of the ovine uterus [330, 331]. Two previous studies also identified enJSRVs RNA and protein in ULF EVs from cyclic and pregnant sheep $[119,120]$. Of note, the enJSRVs Env is critical for conceptus elongation in sheep [319], highlighting the potential important role of EVs in facilitating the transfer of enJSRVs Env protein and other components to the conceptus. GO term analysis of proteins that were differentially abundant in EVs isolated from the ULF of cyclic or pregnant ewes determined that EVs from pregnant ewes were particularly enriched in ribosomal proteins. Factors secreted by the conceptus, such as IFNT and prostaglandins, can directly affect gene expression of the endometrium, modifying uterine histotroph to promote trophectoderm proliferation and attachment [296, 301, 302, 332]. Thus, EVs in pregnant ewes may enhance the ability of the conceptus trophectoderm to translate proteins, increasing the ability of the conceptus to signal to the endometrium and in turn alter the uterine environment. Many of the 289 proteins that were unique to EVs from ULF of pregnant ewes are encoded by genes expressed in the conceptus trophectoderm (e.g. IFNT, pregnancy specific antigen), and many of them overlap with proteins in EVs isolated from media conditioned by day 14 conceptuses cultured ex vivo (e.g. ApoB100, nucleolin, complement 1, complement $\mathrm{C}$, complement $\mathrm{H}$, cathepsin Z) [118]. Fluorescentlylabeled conceptus-derived EVs target the epithelium of the endometrium in sheep 
[118], and thus EVs from the conceptus may be internalized and affect function of the endometrial epithelium.

To understand the origin of EVs in the uterine lumen, the top 10 most abundant proteins in uterine EVs was compared with RNA-seq and qPCR data from uterine epithelia (LE and GE) and the conceptus from day 14 pregnant ewes as well as proteins in EVs isolated from fetal bovine serum and RNA-seq from whole bovine endometrium $[72,140,333,334]$. The uterine GE RNA-seq data had the most overlap with the most abundant EV cargo, suggesting that the uterine glands are the major source of EVs in the uterine lumen. Interestingly, some of the most abundant proteins were identified in RNA sequencing of whole endometrium but not uterine epithelium. Thus, although the stroma is likely not the primary source of EVs, it likely contributes to some of the protein cargo found in uterine EVs. Although conceptus specific proteins were identified in ULF EVs from pregnant ewes, several of these proteins had few spectral counts, suggesting that conceptus-derived EVs represent a smaller proportion of the total EVs present in the uterine lumen of a pregnant ewe. Unexpectedly, bacterial ribosomal protein was present in EVs isolated from ULF of both day 14 cyclic and pregnant ewes. These findings support the idea that there is a resident microbiome in both the cyclic and pregnant uterus, and that there is the potential for these bacteria to contribute to uterine EVs [335, 336].

The lipid cargo of EVs is not well characterized, particularly those isolated from the uterine lumen $[98,99,307]$. In the present study, we found that ULF EVs did not contain cholesteryl esters and triglycerides, which is consistent with EVs 
from other tissues; those lipid populations are more typically associated with lipoproteins and lipid droplets [337-339]. Interrogation of the phospholipid makeup of EVs found that a wide variety of phospholipid types in ULF EVs were similar between day 14 cyclic and pregnant ewes. The exception was that phosphatidylcholine/sphingomyelin and phosphatidylethanolamine phospholipid populations segregated by hierarchical clustering analysis, suggesting that those populations of phospholipids are affected by pregnancy status. Specifically, $\mathrm{PCo}(32: 3)$ and $\mathrm{PE}(36: 1)$ were 3.1-fold and 2.1-fold higher in EVs from cyclic than pregnant animals, respectively, while SM(d16:1/18:1) was 2.7-fold higher in EVs from pregnant animals. These shifts and changes in overall phospholipid makeup could arise from altered lipid composition of conceptus-derived EVs or the altered production of different EV subpopulations from the endometrium due to pregnancydependent changes. The phospholipid populations and fatty acid chains contained within a phospholipid affect the rigidity and shape of the EVs [96, 340, 341]. These overall alterations in phospholipid patterns and specific metabolites may have consequences for EV cargo stability and internalization by recipient cells. Of note, some of the more abundant phospholipids in both cyclic and pregnant EVs contained ether and vinyl-linked fatty acid linkages instead of ester linkages. These fatty acid linkages promote membrane rigidity and may promote EV stability while in the uterine lumen $[342,343]$.

Ceramides are key components of EVs and play important roles in the biogenesis of exosomes. Interestingly, heatmap analysis indicated that EVs from the ULF of pregnant ewes were enriched in the two most overall abundant 
ceramides, C16 Ceramide (d18:1/16:0) and C24 Ceramide (d18:1/24:0), that were 1.6- and 1.8-fold more abundant in pregnant than cyclic ULF EVs, respectively. Medium chain ceramides, such as $\mathrm{C} 16$ and $\mathrm{C} 18$, favor negative budding of the membrane and are implicated in endosomal sorting complexes required for transport (ESCRT)-independent formation of multivesicular bodies and exosome release [84]. Thus, it is possible that alterations in the ceramide populations of EVs may indicate that there is a higher proportion of exosomes in the population of EVs isolated from pregnant flush. Importantly, ceramides can play significant roles in cell signaling and regulation of cell cycle and apoptosis. Accordingly, EV delivery of ceramides may have direct effects on recipient cell biology [344, 345].

The ULF EVs contained FFAs including polyunsaturated fatty acids such as arachidonic acid. Heatmap clustering of FFAs found a shift from medium chain saturated FFAs, including palmitic (C16:0) and stearic acid (C18:0), in cyclic EVs towards unsaturated and/or longer-chained FFAs in pregnant EVs. EVs isolated from cyclic ewes had 2.2-fold higher levels of palmitic acid, while those from pregnant ULF were enriched with more complex fatty acids, such as C30:0,C24:6 (nisinic acid). Thus, endometrial-derived EVs may be loaded to deliver these fatty acids to the developing conceptus. Interestingly, a recent characterization of the FFA makeup in the ULF of heifers reported that palmitic and stearic acid account for approximately $90 \%$ of total free fatty acids [346]. In contrast, EVs contain a much more diverse population of FFAs, with palmitic and stearic acid comprising on average just $44 \%$ of all FFAs observed. Thus, EVs or other lipid complexes may be an important method of FFA delivery to the conceptuses versus uptake of fatty 
acids from the ULF by the conceptus. Moreover, peroxisome proliferator activated receptor gamma (PPARG), a nuclear receptor that binds prostaglandins and other polyunsaturated fatty acids, is expressed in the conceptus trophectoderm and is important for conceptus survival in sheep [206, 222]. FFAs or eicosanoid derivatives delivered via EVs could bind PPARG, altering gene expression in the developing conceptus trophectoderm. Overall, ULF EVs may be an important source of FFA delivery to the developing conceptus. While there are noticeable shifts in several lipid populations by pregnancy status, it is unclear whether these are from intentional loading of specific lipid species or simply are byproducts of shifts in EV biosynthetic pathways. Nevertheless, lipids likely play a critical role in conceptus development due to the demand of cellular proliferation, cell signaling/prostaglandin synthesis, and homeostasis requirements [161, 347]. Altogether, present and previous findings support the hypothesis that EVs in the uterine lumen provide various phospholipids, ceramides, and free fatty acids to the conceptus and have biological roles in its survival, growth and development.

In order to understand how ULF EVs and their cargo impact conceptus development, in vitro studies were performed using mononuclear oTr cells and EVs isolated from the uterine lumen. In sheep, the major gene expression changes in endometrial epithelia that promote conceptus proliferation, growth, and survival occur between days 10 and 12 in response to ovarian progesterone and progesterone also regulates uterine EVs and their cargo [126, 296, 302, 309]. These gene expression changes alter the uterine milieu and promote conceptus elongation, which starts around day 12 and continues through day 17 [19]. Thus, 
the following experiments were designed to use EVs from day 14 cyclic or pregnant ewes to understand how EVs may then be affecting trophectoderm biology during this crucial elongation process after progesterone action has had sufficient time to alter the uterine environment. Of note, there are approximately $7 \times 10^{10}$ total EVs in the uterine lumen of day 14 cyclic ewes. Based on the assumption of a total 500 $\mu \mathrm{L}$ volume of uterine fluid in the lumen, the likely in vivo concentration of EVs is approximately $1.4 \times 10^{11} \mathrm{EV}$ per $\mathrm{ml}$. As such, the in vitro experiments conducted here were based within the physiological range of EVs present in the uterine lumen in vivo (i.e. $1 \times 10^{7}$ to $1 \times 10^{10} \mathrm{EV}$ per $\mathrm{ml}$ ). Intriguingly, EVs from ULF of both day 14 cyclic and pregnant ewes decreased oTr cell proliferation at the $72 \mathrm{~h}$ timepoint. Thus, EVs might represent a possible mechanism in which the endometrium regulates on-time elongation of the developing conceptus. Indeed, transferring an embryo to a synchronized uterus is essential for conceptus development and survival, and asynchrony between the embryo and underlying endometrium result in higher rates of pregnancy loss [140,348,349]. The effects of EVs on oTr cell proliferation here contrast with a previous study finding that EVs increased oTr cell proliferation in vitro [120]. The EVs used in that study were isolated using Exoquick precipitation. As noted previously, precipitation of EVs appears to co-precipitate abundant proteins in the uterine lumen, such as ALB and HGB, that are not actual EV cargo. Moreover, ALB can bind fatty acids and other growth factors [350], which may account for the increase in cellular proliferation observed in that specific study with uterine EVs [120]. The mechanism of how EVs from the uterine lumen of cyclic and pregnant ewes inhibit oTr cell proliferation is not known. Ceramides, which are 
contained in EVs from both cyclic and pregnant uterine flushes, can activate a stress-activated protein kinase cascade to induce cell cycle arrest and prevent proliferation of a variety of cancer cells in vitro [351-353]. Thus, it is perhaps the delivery of ceramides in uterine EVs to the cells that prevents their proliferation.

Another finding of the present study was that day 14 cyclic and pregnant ULF EVs did not affect gene expression in oTr cells in vitro as determined by comprehensive RNA-seq and candidate gene expression analyses. This finding was unexpected, as the ULF EVs from cyclic and pregnant ewes contain a plethora of miRNAs and some mRNA $[118,119,126]$. Although IFNT mRNA levels were unaffected by EV treatment, the production of IFNT protein was increased by treatment of oTr cells with ULF EVs in the present study. Similarly, ULF EVs increased IFNT production by oTr cells in a previous study [120]. Given that inert control liposomes did not increase IFNT in the culture media, the cargo or lipid component of EVs must increase IFNT protein production by the oTr cells. Indeed, the inert control liposomes do not contain any miRNA or protein cargo and they are only comprised of phosphatidylcholine and cholesterol. Proteomics analysis found that EVs isolated from the uterine lumen of both day 14 cyclic and pregnant ewes contained ribosomal proteins with higher amounts those from pregnant ewes. It may be possible that the provision of ribosomes by the EVs to the conceptus trophectoderm enhances production of proteins such as IFNT. Consistent with this, ribosome recruitment to axons occurs via lateral transfer from glial cells via exosomes [354]. Alternatively, the lipid cargo of uterine EVs could affect protein production by $\mathrm{OTr}$ cells. Lipids play critical roles in regulation of 
secretory granule biogenesis and release [355]. Although it appears EVs may have minimal effects on gene expression of the conceptus trophectoderm, EVs may increase the synthetic or secretory capacity of the developing conceptus, and thus mediate signaling between the developing conceptus and endometrium.

In summary, the results here support the overall hypothesis that EVs, including exosomes and microvesicles, from the endometrium and conceptus are present in the uterine lumen, are a mode of intercellular communication, and regulate conceptus growth and development via effects on the trophectoderm. Uterine EVs contain a diverse population of proteins and lipids which may affect growth and development of the conceptus, which likely can affect recipient cells once internalized. Notably, EVs appear to decrease proliferation of oTr cells and promote secretion of IFNT without effects on gene expression. Thus, the protein and/or lipid cargo may have more important roles in conceptus development versus the nucleic acid cargo (e.g. RNAs) of EVs.

\section{Acknowledgements}

The authors greatly appreciate David Todd for animal care, and Brian Mooney and the University of Missouri Charles W. Gehrke Proteomics Center and Nathan Bivens and the University of Missouri DNA Core Facility for technical assistance with sample analysis. This work was supported by AFRI 2015-67015-23678 and 2016-67015-24741 grants from the USDA National Institute of Food and Agriculture. 


\section{Supplemental Information}

Supplemental Table 1. Proteome of uterine EVs isolated from day 14 cyclic and pregnant ewes.

Supplemental Table 2. Lipidome of uterine EVs isolated from day 14 cyclic and pregnant ewes.

Supplemental Table 3. Lipids different between uterine EVs isolated from day 14 cyclic and pregnant ewes.

Supplemental Table 4. RNA-sequencing analysis of ovine trophectoderm cells after treatment with liposomes or uterine EVs isolated from day 14 cyclic or pregnant ewes.

Supplemental Figure 1. Real-time qPCR analysis of PTGS2 and IFNT in ovine trophectoderm cells after treatment with liposomes or uterine EVs isolated from day 14 cyclic or pregnant ewes.

\section{Available online at:}

https://academic.oup.com/biolreprod/articlelookup/doi/10.1093/biolreprod/ioaa019\#supplementary-data 


\section{CHAPTER III: \\ EXTRACELLULAR VESICLE EFFECTS ON THE ENDOMETRIUM OF SHEEP}

Eleanore V. O'Neil, Susanta Behura, and Thomas E. Spencer

This work is under preparation for submission to Biology of Reproduction. 


\begin{abstract}
In sheep and other domestic species, early pregnancy events require crosstalk between the developing conceptus and underlying endometrium. Extracellular vesicles (EV) are present within the uterine luminal fluid, originate from the conceptus and endometrium, and may mediate conceptus-endometrium communication. Uterine EVs have a myriad of effects on the conceptus, including regulation of trophectoderm proliferation and interferon tau (IFNT) production. In the present study, liposomes or EVs isolated from the uterine lumen of day 14 cyclic (CEV) or pregnant ewes (PEV) were infused into the uterine lumen of cyclic ewes. Infusion of CEV and PEV altered the endometrial transcriptome of cyclic ewes with predicted effects on metabolic processes and cellular translation among others. Although endometrial PTGS2 expression was not different between CEV and PEV treated ewes, fewer total prostaglandins were recovered from the uterine lumen of PEV infused ewes. Although CEV and PEV infusion increased expression of ESR1 in the endometrium, ESR1 was less abundant in the LE of PEV treated ewes, possibly indicating a potential role for PEV in IFNT pregnancy recognition signaling. Taken together, these results support the hypothesis that EVs act on the endometrium during early pregnancy in sheep.
\end{abstract}




\section{Introduction}

Pregnancy establishment requires interactions and crosstalk between the developing embryo and the underlying endometrium [296, 356, 357]. After the embryo enters the uterus as a morula around day 6 (day $0=$ estrus), the embryo develops into a blastocyst and hatches from the zona pellucida by day $8[20,358]$. The hatched blastocyst forms a spherical or ovoid shaped conceptus (embryo and its associated extraembryonic membranes) and, by day 12 , begins to elongate. Coincident with elongation, the conceptus produces and secretes prostaglandins (PG) and interferon tau (IFNT), the maternal recognition of pregnancy signal, which prevents production of luteolytic pulses of prostaglandin F2 alpha (PGF2 $\alpha$ ) by the endometrium to maintain the corpus luteum and progesterone secretion [216, 359]. By day 16 , the conceptus is fully elongated and begins to attach and firmly adhere to the uterine epithelium to initiate placentation [296, 302, 356, 357].

Conceptus elongation and subsequent embryo development is regulated by the uterine environment, as embryos can develop to the blastocyst stage and hatch from the zona pellucida in vitro, but must be transferred to recipient synchronized ewes to develop past this point $[64,65,360]$. Expanding upon this, studies using the uterine gland knockout (UGKO) ewe model found that endometrial epithelia, and by inference, their products and secretions, are required for conceptus elongation [6, 67, 68]. These endometrial secretions are collectively termed histotroph and are a mixture of lipids, protein, amino acids, cytokines, and carbohydrates among other factors $[72,119,138,357,361]$. 
Exosomes and microvesicles, collectively termed extracellular vesicles $(\mathrm{EV})$, are also a component of histotroph and are a heterogeneous mixture of cellderived vesicles released into the extracellular space and taken up by recipient cells. Growing evidence suggests that EV are mediators of intercellular communication in many biological systems as their cargo, including lipids, protein, and nucleic acids (such as mRNAs and miRNAs) can affect recipient cell function or biology [362, 363]. Notably, EV are present in the uterine lumen and produced by both the endometrial epithelium and conceptus trophectoderm [118, 119, 125]. Endometrial production of EV is regulated by progesterone and increases at the onset of conceptus elongation, although EV are less abundant in the uterine lumen of pregnant ewes likely due to uptake by the conceptus [126, 364]. Growing evidence suggests that EV play important roles during conceptus elongation as EV deliver endogenous retroviral elements and regulate trophectoderm cell proliferation and IFNT production [119, 120,364]. While most experiments have focused on how uterine EV may affect trophectoderm cell processes, EV can also be internalized by the endometrial epithelium and are hypothesized to alter gene expression and epithelial secretions $[118,125]$. In the present study, liposomes or EV from day 14 cyclic or pregnant ewes were infused into the uterine lumen of ewes between days 10 and 14 of their estrous cycle to understand effects of EVs on the endometrium.

\section{Materials and Methods}

\section{Animals}


All experimental and surgical procedures for mature sheep (Ovis aries) were approved by the Institutional Animal Care and Use Committee of the University of Missouri. Adult cycling ewes were checked daily for estrus (day 0) and euthanized on day 14 of the estrous cycle $(14 C)(n=5)$ or day 14 of pregnancy after mating to an intact ram of proven fertility (14P) $(n=5)$. The entire female reproductive tract was obtained through a mid-ventral incision, and the broad ligament trimmed off. A clamp was placed on the internal ostium of the cervix, and the oviduct and uterotubal junction was removed. A catheter attached to a syringe containing 10-ml sterile PBS ( $\mathrm{pH} 7.2)$ was inserted about $1 \mathrm{~cm}$ into the uterine horn contralateral to the corpus luteum. Sterile PBS was slowly instilled into the uterine lumen and then gently massaged through the uterine body into and through the ipsilateral horn that was held over a sterile petri dish using a hemostat. The uterine lumen flush (ULF) was collected in the petri dish and the conceptus was removed if present. The ULF was then clarified by centrifugation $(3000 \times g$ for $15 \mathrm{~min}$ at $4^{\circ} \mathrm{C}$ ), transferred into a new $15-\mathrm{ml}$ tube, and volume recorded prior to storage at $80^{\circ} \mathrm{C}$ for $\mathrm{EV}$ isolation. The endometrium was physically dissected from the myometrium and flash frozen in liquid nitrogen for RNA extraction.

Next, ewes $(n=15)$ were detected for estrus using a vasectomized ram. Using a surgical approach described previously [69, 216, 364], ewes on day 10 post-estrus were subjected to a midventral laparotomy, and the lumen of each uterine horn received a vinyl catheter (0007760; Durect Corp, Cupertino, California) connected to an Alzet 2ML1 osmotic pump (Durect Corp) secured by suturing the oviduct and mesovarium to the outside of the uterus. Ewes ( $n=5$ per 
treatment) received pumps containing the following: 1) $2 \mathrm{~mL}$ of saline vehicle containing $2 \times 10^{10}$ liposome particles; 2) $2 \times 10^{10} \mathrm{CEV}$; 3) $2 \times 10^{10} \mathrm{PEV}$ (Figure 1). The Alzet 2ML1 osmotic pump has a reservoir volume of $2 \mathrm{~mL}$ and a pumping rate of $10 \mu \mathrm{L} / \mathrm{h}$ for 7 days. At necropsy on day 14 after estrus, the female reproductive tract was excised, and ULF and endometrium obtained as described previously. Additionally, a section from the middle of the uterine horn ipsilateral to the CL was fixed in $4 \%$ paraformaldehyde in PBS $(\mathrm{pH} \mathrm{7.2)}$ at room temperature for $24 \mathrm{~h}$, dehydrated through a series of ethanol and embedded in paraffin for histology.

Extracellular vesicle isolation by size exclusion chromatography (SEC) and quantification by nanoparticle tracking analysis (NTA)

Isolation of EV was performed as previously described [126, 364]. Briefly, clarified ULF was thawed on ice and filtered through a 0.22- $\mu \mathrm{m}$ PVDF syringe filter (cat \# SLGV033RS, EMD Millipore, Billerica, MA). Filtered ULF was concentrated by ultrafiltration with Centricon Plus-70 centrifugal filter units (cat \# UFC710008, EMD Millipore) and brought to $500 \mu \mathrm{l}$ by weight with sterile PBS. The EVs were isolated from the concentrated flush by SEC using qEVoriginal columns (Izon Science Ltd, Oxford, UK). Columns were equilibrated with $20 \mathrm{ml}$ of PBS prior to running the sample through the column and collecting fractions 7 to $9(1.5 \mathrm{ml}$ total). These fractions were reconcentrated with an Amicon Ultra-4 device (cat \# UFC801008, EMD Millipore), and brought to $200 \mu \mathrm{l}$ with sterile PBS. Aliquots of recovered EVs were diluted to approximately $10^{7}-10^{8}$ particles per $\mathrm{ml}$ with $5 \mathrm{mM}$ ethylenediaminetetraacetic acid (EDTA) in PBS and the evaluated by NTA using a NanoSight NS300 instrument (NanoSight Ltd, Amesbury, UK) equipped with a 
low-volume flow cell and syringe pump. The NanoSight was calibrated with 100 $\mathrm{nm}$ polystyrene beads (Polysciences, Warrington, PA). Videos were captured (camera level $13-14$, syringe pump speed 30 , temperature control setting $25^{\circ} \mathrm{C}$ ) using the standard measurement protocol of five $60 \mathrm{sec}$ videos followed by processing with NTA software (NanoSight) to track each visible particle. The Stokes-Einstein equation was employed by the software to determine the size distribution and number of particles (concentration) within each sample.

Total RNA isolation and analysis

Frozen endometrium was homogenized in TRIzol reagent (Thermo Fisher Scientific) and total RNA was isolated using a Direct-zol RNA MiniPrep Plus isolation kit (Zymo Research, R2070) on EconoSpin Columns (Epoch Life Science, Inc. Missouri City, TX). Samples were treated with an on-column DNAse I digestion and run through RNeasy MinElute cleanup columns (Qiagen). Quantity of total RNA was determined by Qubit RNA BR Assay and run on an agarose gel to assess RNA quality.

\section{RNA sequencing}

RNA library preparation and sequencing of endometrium was conducted by Novogene (Sacramento, California). The raw sequences (FASTQ) were subjected to quality check by FastQC http://www.bioinformatics.babraham.ac.uk/projects/fastqc/. The program fqtrim https://ccb.jhu.edu/software/fqtrim/ was used to remove adapters, perform quality trimming (phred score $>30$ ) by a sliding window scan ( 6 nucleotides) and select read length of 30 nucleotides or longer after trimming. The 
reads were mapped to the Ovis Aries reference genome (Oar_V3.1) using the Hisat2 aligner. FeatureCounts was used to quantify read counts [324]. Differentially expressed (DE) genes were identified by edgeR-robust [325]. The false discovery rate $(F D R)<0.05$ was used as threshold for statistically significant differential expression of genes. Enrichment analysis of DE genes (FDR P-value $\leq$ 0.05 , Fold change $\geq 2$, Expression $\geq 1$ FPKM) was conducted using GOTERMFINDER https://go.princeton.edu/cgi-bin/GOTermFinder and REVIGO http://revigo.irb.hr/.

\section{Quantification of Prostaglandin}

Total PG in the uterine flush was measured by PG Screening EIA Kit from Cayman Chemical (514012; Ann Arbor, Michigan) as previously described [222, 359]. This assay detects almost all PGs (PGE1, PGE2, PGF1 $\alpha$, and PGF2 $\alpha$ ) with $100 \%$ cross-reactivity.

\section{Estrogen receptor 1 (ESR1) Staining}

Uteri were sectioned at $8 \mu \mathrm{m}$ and allowed to air dry for 10 minutes at $37^{\circ} \mathrm{C}$. Sections were dewaxed in xylene and a series of ethanol and $\mathrm{dH}_{2} \mathrm{O}$. Antigen recovery was done using a Reveal Decloaker (ThermoFisher Scientific) at $100^{\circ} \mathrm{C}$ for $30 \mathrm{~m}$. Sections were cooled in Decloaker reagent in an ice bath for $30 \mathrm{~min}$ and were then permeabilized using PBST (PBS with $0.1 \%$ Tween 20) for 20 minutes at room temperature (RT) before washing with PBS and then Milli-Q $\mathrm{H}_{2} \mathrm{O}$ to remove excess salts. Next, uterine sections were washed twice with PBS for 5 minutes each and blocked with 10\% normal goat serum (Life technologies, 50062Z) for 30 
minutes at RT. Sections were incubated with primary antibodies against ERa (1:100 dilution; C-3, mouse monoclonal antibody, Santa Cruz Biotechnology) and E-cadherin (1:200 dilution; 24E10, rabbit monoclonal antibody, Cell Signaling Technology) overnight at $4^{\circ} \mathrm{C}$ in $10 \%$ normal goat serum. The following day, sections were washed twice with PBS, 10 minutes each and incubated with Alexa 555-conjugated secondary antibody (1:400; Goat anti-Mouse IgG Invitrogen A32727) and Alexa 488-conjugated secondary antibody (1:400 dilution; Goat antirabbit Invitrogen, A32731) and for 1 hour at RT. Sections were washed twice with PBS for 5 minutes each followed by Milli-Q $\mathrm{H}_{2} \mathrm{O}$ for 10 minutes. Sections were counterstained with Hoechst (1:5000 dilution in Milli-Q $\mathrm{H}_{2} \mathrm{O}$; Invitrogen, $\mathrm{H} 3570$ ) for 5 minutes then washed with Milli-Q $\mathrm{H}_{2} \mathrm{O}$ for 5 minutes and mounted with FlouroMount-G Mounting medium (ThermoFisher Scientific). Fluorescent images were collected with a Leica DM5500 B upright microscope using the Leica Application Suite X (LAS X).

To quantify fluorescence, images were analyzed using ImageJ software. Briefly, images taken in the red-fluorescent channel were converted to an 8-bit grayscale format and the red fluorescence were segmented using thresholding. Threshold conditions were kept the same across all uterine sections. Next, image thresholding and quantification was repeated using Hoeschst-blue stained nuclei to account for total cells. Staining for ESR1 was then calculated as a ratio to total nuclei and compared between treatments. To quantify ESR1 abundance in the LE specifically, images were converted to RGB stacks and E-cadherin staining was 
used to manually isolate LE cells and crop out all other cell types. Staining of ESR1 was then completed the same way for the entire uterine section.

\section{Statistics}

All quantitative data were subjected to least-squares ANOVA using the GLM procedures of the SAS (SAS Institute Inc., Cary, NC). In all analyses, error terms used in significance were identified according to the expectation of the mean squares for error. Significance $(P \leq 0.05)$ was determined by the probability differences of the least-squares means.

\section{Results}

Pump infusion of all three treatments altered endometrial gene expression relative to untreated Day 14 cyclic ewes (Figure 1A) (Supplementary Table 1). Seven genes and one gene were increased and decreased, respectively, by all three treatment groups compared to a day 14 cyclic ewe (Figure 1B). Infusion of liposomes alone increased 24 genes and decreased 12 genes.

The most noticeable changes in gene expression were observed in ewes who received infusion of CEV. In total, 221 genes were increased, including PTGS2 and ESR1, and 33 were decreased, including ADAMTS1. To understand the potential functional consequences of these transcriptome changes, differentially expressed genes were entered into gene ontology (GO) analysis. Notably, infusion of CEV increased expression of genes involved with positive gene transcription, regulation of primary metabolism, and cell differentiation 
(Figure 2A) among other processes (Supplementary Table 2A) relative to the nontreated cyclic ewes. Conversely, CEV infusion decreased genes that regulate nonsense mRNA-mediated decay, protein localization to the endoplasmic reticulum, and translational initiation (Figure 2B) among others (Supplementary Table 2B).

A



B



Figure 1. Summary of endometrial transcriptome analyses. (A) Differentially expressed genes (FDR, $\mathrm{P}<0.05)$ were determined by edgeR robust analysis and are listed in black. The total number of increased and decreased transcripts are shown in red and blue, respectively, for each comparison. (B) Venn diagram showing the number of unique or common differentially expressed transcripts upregulated or downregulated by each treatment (Liposomes, CEV, or PEV) when compared to untreated day 14 cyclic ewes. CEV, extracellular vesicles from a day 14 cyclic ewe; PEV, extracellular vesicles from a day 14 pregnant ewe; FDR, false discovery rate. 
A



Log10 P-Value

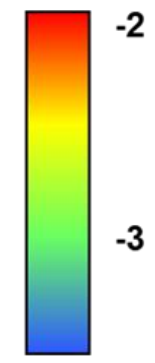

Log size

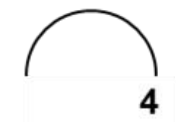

B



Log10 P-Value

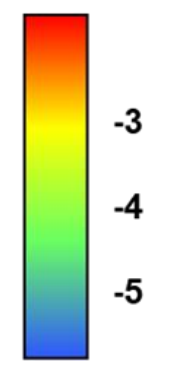

Log size

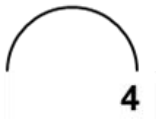

Figure 2. Enrichment analysis of differentially expressed transcripts in CEV infused ewes. Gene ontology analysis was visualized using REViGO software. The scatterplot places GO terms in a 2D plot to cluster processes with semantic similarities and removes functionally redundant GO terms. Color of the circle indicates the FDR $P$ value of the GO term and size of the circle indicates the frequency of the GO term in the underlying annotation database. (A) Genes increased by CEV infusion were enriched for processes such as regulation of primary metabolism and cell differentiation. (B) Genes decreased by CEV infusion overrepresented processes including protein localization to endoplasmic reticulum and translational initiation. CEV, extracellular vesicles from a day 14 cyclic ewe; FDR, false discovery rate; GO, gene ontology; PEV, extracellular vesicles from a day 14 pregnant ewe. 
Fewer transcriptome changes were observed in ewes who received PEV infusion than CEV; 35 genes were increased, such as ESR1 and CXCL14, and 17 were decreased including ADAMTS1. When these genes were inserted into gene ontology analysis, the only process identified was an increase in iron assimilation processes. Infusion of either EV treatment increased expression of 18 genes and decreased expression of 4 genes.

Although infusion of CEV increased PTGS2 compared to cyclic ewes, PG were not elevated in the uterine lumen. Further, CEV-infused ewes had more PG in their uterine lumen than PEV-infused ewes although PTGS2 expression was not different between these groups (Figure 3). To ensure that PG possibly contained in EV did not account for the results here, $1 \mathrm{E}+10$ of CEV or PEV (the approximate amount of EV infused by osmotic pumps between days 10 and 14) were suspended in PBS and total PG were measured. In total, $1 E+10$ CEV only contained $0.9 \pm 0.5 \mathrm{ng}$ of $\mathrm{PG}$ and $1 \mathrm{E}+10 \mathrm{P}$ EVs contained $3.16 \pm 1.1 \mathrm{ng}$ of PG. 


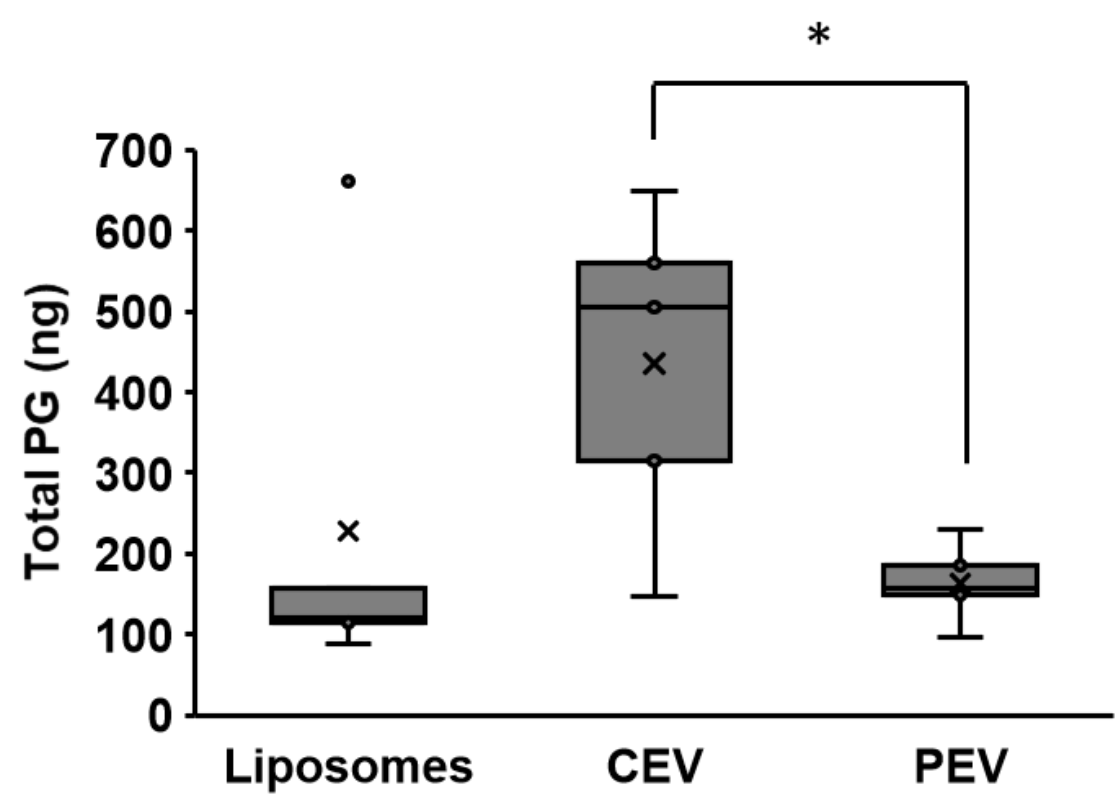

Figure 3. Prostaglandins were elevated in the uterine lumen of CEV infused ewes compared to PEV infused ewes. Asterisk denotes statistical significance $(P<0.05)$. CEV, extracellular vesicles from a day 14 cyclic ewe; PEV, extracellular vesicles from a day 14 pregnant ewe.

Finally, ESR1 abundance was quantified in the three treatment infused groups by immunofluorescence since both CEV and PEV had higher expression of ESR1 than nontreated cyclic ewes (Figure 4A). Notably, ESR1 was less abundant in entire uterine sections (Figure 4B) and the LE (Figure 4C) of PEV treated ewes when compared to both liposome and CEV infused ewes although gene expression between these groups was not different. 


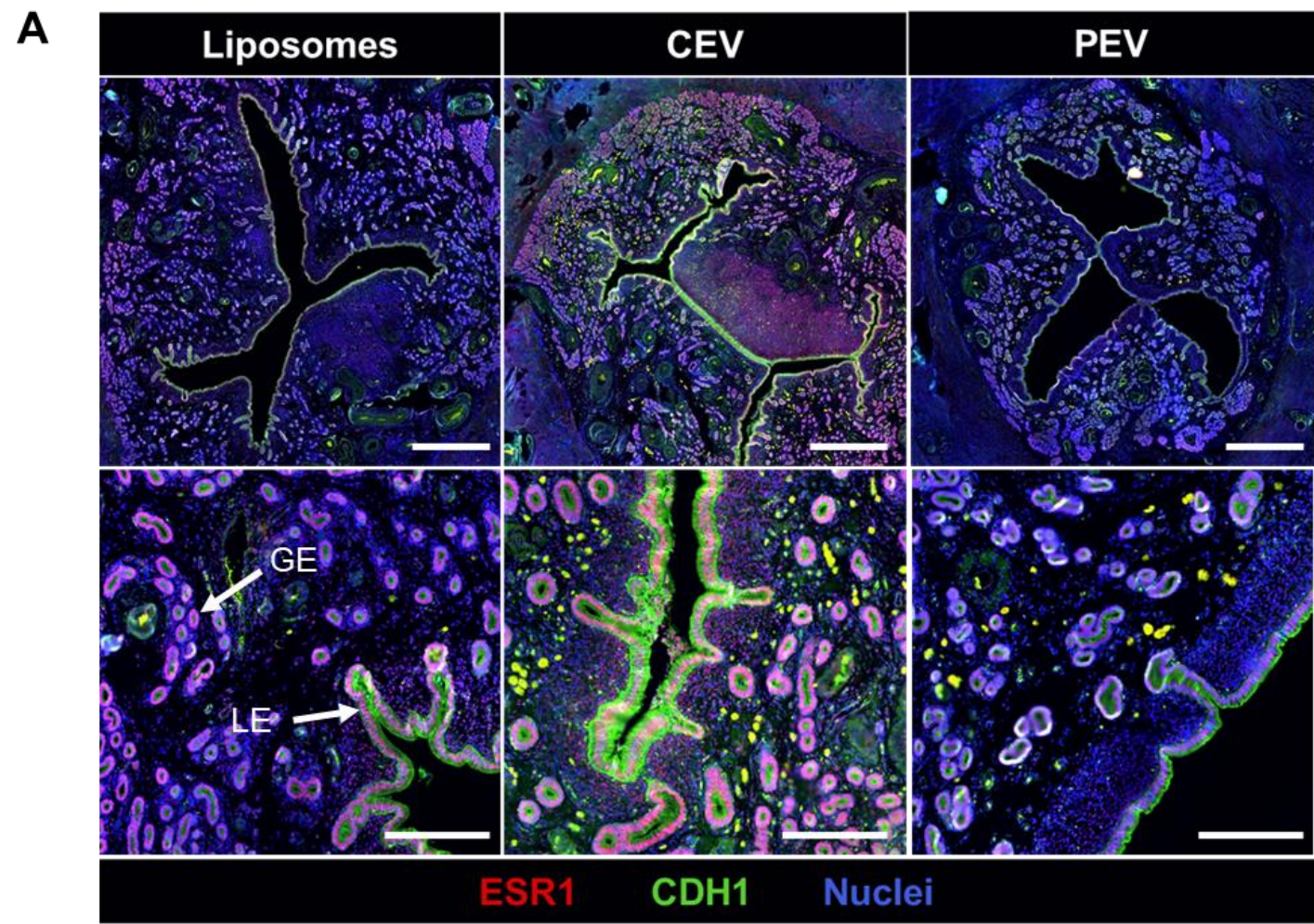

B

Uterine section

*

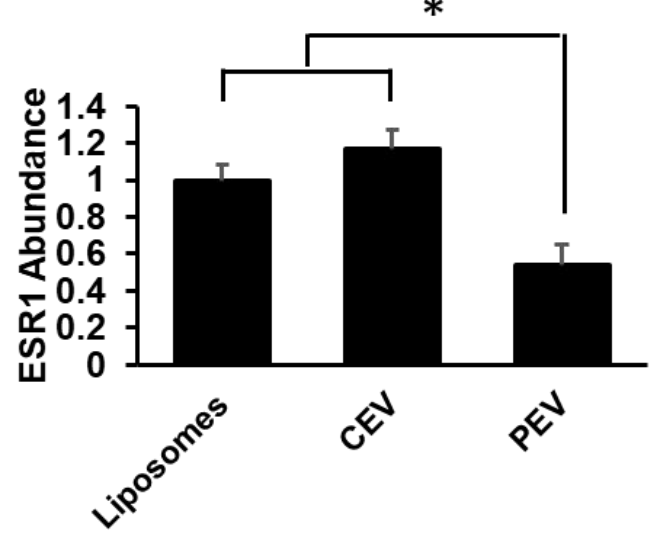

C

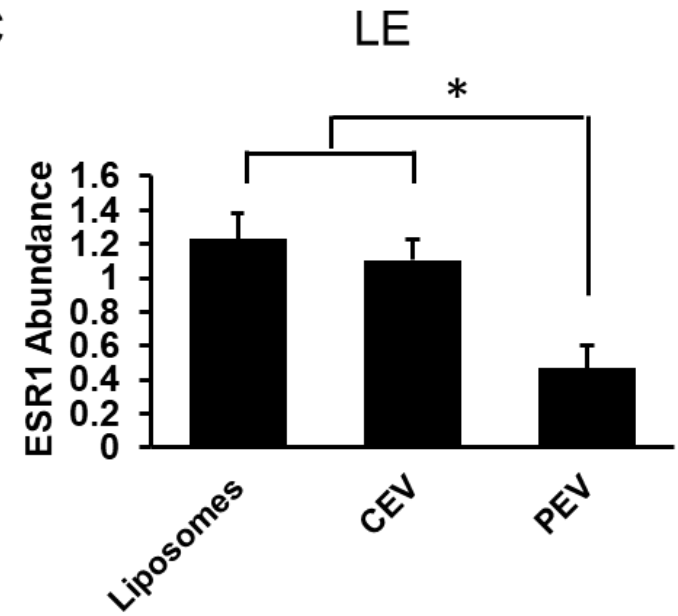

Figure 4. Infusion of PEV prevented upregulation of uterine ESR1. (A) ESR1 and CDH1 localization in liposome, CEV, and PEV infused uteri. Scale bar: 1000 $\mu \mathrm{m}$ and $250 \mu \mathrm{m}$ for top row and bottom row, respectively. (B) When normalized to DAPI fluorescence, ESR1 was less abundant in PEV infused ewes than liposome or CEV infused ewes. (C) Staining of E-cadherin was used to manually isolate and measure ESR1 in the LE, which was also lower in PEV infused ewes. CEV, extracellular vesicles from a day 14 cyclic ewe; CDH1, epithelial cadherin; ESR1, estrogen receptor 1; PEV, extracellular vesicles from a day 14 pregnant ewe. 


\section{Discussion}

The results here support the hypothesis that EV play important biological roles in endometrial regulation and mediate intercellular communication during early pregnancy. Some studies have reported that EV may upregulate endometrial adhesion factors and IFN-stimulated gene expression, increase endometrial vasculogenesis, and suppress the maternal immune response $[121,135,136]$. However, these studies were conducted in vitro using endometrial epithelial and endothelial cell lines and may not accurately recapitulate the biological effects of EV on the endometrium. In the present study, the effects of CEV and PEV on the endometrium was determined in vivo.

To understand how EV may affect the endometrium, EV were quantified prior to infusion and were normalized so that the same total number of liposomes or EV ( $1 \times 10^{10}$ particles per $\mathrm{ml}$ of PBS) was infused into the uterine lumen of all ewes. This was done to correct for EV concentration effects, as pregnant ewes have approximately half the total number of EV in the uterine lumen compared to cyclic ewes on day 14 . The concentration used is physiologically relevant and has been previously used for in vitro experiments [364]. Of note, infusion of all three treatments upregulated seven common genes, such as uroplakin $1 \mathrm{~B}(U P K 1 B)$ and calpain 8 (CAPN8), and downregulated one common gene. Liposomes are inert vesicles that contain no cargo and are made of phosphatidylcholine (PC) and cholesterol, indicating that either the surgery, pump infusion of a solution, or the infusion of PC/cholesterol lipids accounted for the changes in endometrial gene expression. Liposomes alone altered 21 unique genes indicating that liposome 
imparts their own effects on the endometrial transcriptome [365, 366]. Consistent with this, some genes were affected by liposomes and only one of the EV treatments; thus, how the presence and concentration of EV-contained cholesterol or PC differentially affects gene expression is worth further study [367-369].

As expected, infusion of CEV and PEV altered endometrial expression more than liposomes. Infusion of CEV upregulated many genes, such as solute carrier 7 member 5 (SLC7A5) and ELOVL fatty acid elongase 2 (ELOVL2), that are involved in macromolecule, nitrogen compound, and cellular metabolism processes as well as cell differentiation and development. In cyclic ewes, there is an increase in amino acids, glucose, and other metabolites in the uterine lumen around day 14, likely to make metabolic substrate available in case of pregnancy $[138,139,145]$. Therefore, CEV may promote endometrial synthesis of metabolic substrate and/or metabolite transportation into the uterine lumen. Of note, $R A B 27 B$, a key regulator of EV release, was also upregulated by CEV infusion, suggesting that CEV may positively regulate their own secretion into the uterine lumen $[85,370]$. Endometrial genes that were downregulated by CEV were largely related to the regulation of $\mathrm{mRNA}$ and protein translation, including nucleolar proteins and several ribosomal components. Ribosomal mRNA and proteins are overrepresented in both CEV and PEV, so it is unclear if CEV specifically target these genes for downregulation or if their downregulation occurs in response to the acquisition of translational machinery from CEV $[118,364]$. Since these gene expression changes were observed in day 14 cyclic ewes in response to the infusion of CEV from day 14 ewes, it is evident that the number of EV plays a 
critical role in mediating their biological effect. The total dose of EV was shown to play an important role in in vitro studies using the ovine trophectoderm cells and has been shown in many other systems and highlights the need for more work in understanding the kinetics of EV release, exposure, and uptake [364, 371-373]. Furthermore, it is worth investigating whether insufficient EV production could be an underlying cause of pregnancy loss due to intercellular communication failure.

Infusion of PEV affected more genes than liposome infusion but fewer genes than CEV infusion. In contrast, PEV treatment had similar or greater biological effects than CEV on ovine trophectoderm cells [364]. This discrepancy could be because different biological effects are being compared here (i.e. changes in the endometrial transcriptome vs. cellular proliferation and protein production in ovine trophectoderm cells) or possibly indicates that PEV have greater biological roles in the conceptus than they do in the endometrium. Indeed, CEV and PEV contain different lipid and protein composition that can differentially affect their rate of uptake by the conceptus and endometrium, and the low abundance of PEV in the uterine lumen of pregnant ewes is largely attributed to conceptus uptake [364, 374, 375]. Overall, iron assimilation was the only process found to be upregulated in the endometrium of PEV-infused ewes. Many ions, including $\mathrm{Na}+, \mathrm{K}_{+}$, and $\mathrm{Ca} 2+$ are elevated and thought to play important roles in early embryo development $[138,376]$. Thus, PEV may be geared toward modulating the iron content of the uterine lumen.

Since transcriptome data does not always translate into changes at the protein level, a few differentially expressed candidate genes in the endometrium 
were investigated at a more functional level in the present study [377]. Prostaglandin-Endoperoxide Synthase 2 (PTGS2) was upregulated in CEVinfused ewes and is the rate limiting step in PG biosynthesis [378]. A threshold amount of PG are required for conceptus elongation and PG play key roles in regulating endometrial gene expression and are thus key molecules in pregnancy [216, 359]. Liposome and CEV treated ewes had similar amounts of PG within the uterine lumen but had more PG in the uterine lumen than ewes who received PEV infusion. So, the upregulation of PTGS2 in CEV-infused ewes did not translate into increased production of PG. Furthermore, PEV did not affect PTGS2 expression but decreased PTGS2 activity or PG secretion at a post-transcriptional level. As PTGS2 expression is higher in pregnant ewes than cyclic ewes and PG play important roles in pregnancy, this result was unexpected and warrants further investigation [216, 223]. Additionally, future studies should study whether PEV affect PG production by the conceptus as well or whether these findings are specific to the endometrium. To ensure that these results were not due to PG contained in the EV that were infused, PG were quantified in EV. This result shows the first results that prostaglandins are present in uterine EV, consistent with EV from other biological systems, and may be one way PG are transported between the conceptus and endometrium [99].

In sheep, ESR1 is a key point of regulation for pregnancy establishment. Stimulation of ESR1 by estrogen upregulates the oxytocin receptor (OXTR) which in turn stimulates the pulsatile secretion of PGF2 $\alpha$ to cause luteolysis $[44,45,379]$. Notably, while both CEV and PEV infusion increased ESR1 expression relative to 
a day 14 cyclic ewe, ESR1 was less abundant in PEV-infused ewes than both liposome and CEV infused ewes. Thus, PEV may prevent the upregulation of ESR1 in the LE despite its stimulation in the endometrium at the transcript level. Indeed, IFNT, which prevents the upregulation of ESR1 to rescue the CL, is present in PEV and likely accounts for the results here [364]. Although ESR1 was less abundant in PEV infused ewes, OXTR expression was not different between ewes. As OXTR is not upregulated in the endometrial epithelium until around day 14 or 15 , it is possible that the timing of the current experiment was too early to capture upregulation of OXTR and differences between the groups of ewes [380]. Future studies could investigate whether uterine infusion of PEV delays the start of a new estrous cycle in ewes.

Overall, EV seem to regulate the endometrium during early pregnancy. While EV do not affect ovine trophectoderm gene expression, the endometrium is susceptible to EV regulation at the gene expression level [135, 364]. While CEV seems to predominantly alter the endometrial transcriptome, PEV seemed to have largely inhibitory roles in the endometrium beyond the gene expression level, as PG were less abundant in the uterine lumen and ESR1 was less abundant in uterine sections, possibly indicating roles in $\mathrm{CL}$ rescue. Overall, more work needs to be done on the kinetics of EV uptake and release by the endometrium. Although both CEV and PEV affect the endometrium, it is unclear in pregnancy how many EV are internalized by the endometrium versus the conceptus which would help clarify the directionality of EV transfer and the roles they may have in conceptus- 
endometrial crosstalk. The results reported here support the hypothesis that EV play important roles in endometrial regulation in early pregnancy.

\section{Acknowledgements}

We thank David Todd for his expert assistance with sheep husbandry and experiments.

\section{Supplemental Information}

Supplemental Table 1. RNA-sequencing analysis of endometrium after exposure to liposomes or uterine EVs isolated from day 14 cyclic or pregnant ewes.

Supplemental Table 2. Enriched gene ontology analysis results of differentially expressed genes in endometrium treated with uterine EVs isolated from day 14 cyclic ewes.

\section{Available upon request or after publication at}

http://www.biolreprod.org/ 


\title{
CHAPTER IV:
}

INSIGHTS INTO THE LIPIDOME AND PRIMARY METABOLOME OF THE UTERUS FROM DAY 14 CYCLIC AND PREGNANT SHEEP

\author{
Eleanore V. O'Neil and Thomas E. Spencer
}

This work has been submitted for publication in Biology of Reproduction. 


\begin{abstract}
In ruminants, conceptus elongation requires endometrial products and secretions. The amino acid, carbohydrate, and protein composition of the uterine lumen during early pregnancy has been described in sheep; however, a comprehensive understanding of metabolomic changes in the uterine lumen is lacking, particularly with respect to lipids. Here, the lipidome and primary metabolome of the uterine lumen, endometrium, and/or conceptus was determined on day 14 of the estrous cycle and pregnancy. Lipid droplets and select triglycerides were depleted in the endometrium of pregnant ewes. In contrast, several phospholipid classes (phosphatidylcholine, phosphatidylinositol, phosphatidylglycerols, and diacylglycerols) and select triglycerides, ceramides and non-esterified fatty acids were elevated in the uterine lumen of pregnant ewes. Lipidomic analysis of the conceptus revealed that triglycerides are especially abundant within the conceptus. Primary metabolite analyses showed elevated luminal amino acids, carbohydrates, and energy substrates, among others, during pregnancy. Collectively, this study clarifies the lipidomic and primary metabolic substrates in the ovine uterus, and supports the hypothesis that lipids are components of the uterine lumen, important for conceptus elongation and growth during early pregnancy.
\end{abstract}




\section{Introduction}

In domesticated ruminants (i.e., sheep, cattle, goats), pregnancy establishment encompasses maternal pregnancy recognition, implantation, and placentation [19, 55, 301, 357]. In sheep, the spherical embryo enters the uterus as a morula or early blastocyst, hatches from the zona pellucida (day 8 postmating) and develops into a conceptus (embryo and associated extraembryonic membranes) by day 10 . Beginning on day 12 , the now ovoid conceptus begins to undergo elongation, a series of morphological changes driven by cellular proliferation, resulting in an exponential increase in cell weight and size [19]. By day 14 the conceptus is filamentous in shape and can reach $12-14 \mathrm{~cm}$ in length. During elongation, the conceptus secretes copious amounts of interferon tau (IFNT), the maternal pregnancy recognition signal, which acts on the endometrium to inhibit production of luteolytic pulses of prostaglandin F2 alpha, thereby maintaining the corpus luteum (CL) and securing progesterone production $[300$, 381]. Subsequent implantation and placentation begin around day 16, after the conceptus finishes elongating and occupies the entire length of the uterine horn ipsilateral to the CL.

Although embryos can develop to the blastocyst stage and hatch from the zona pellucida in vitro, hatched blastocysts must be transferred to a synchronized recipient ewe to elongate $[64,65]$. The uterine gland knock out (UGKO) sheep model established that endometrial glandular epithelial secretions are required for the uterus to support conceptus elongation $[67,68]$. These data suggest that endometrial epithelial secretions are essential regulators of conceptus elongation 
and pregnancy establishment $[6,125]$. Uterine secretions, collectively termed histotroph, are a complex mixture of enzymes, metabolites, extracellular vesicles, and other molecules $[119,138,145,147,156,382]$. Many of these components regulate cell proliferation, migration, and attachment, as well as maintain cellular homeostasis, which are critical for conceptus elongation [178-180, 364]. Further, factors from the conceptus, such as IFNT and prostaglandins, actively modify uterine luminal secretions via altering the expression of various solute transporters and other enzymes in the endometrial epithelium [383, 384].

Available data suggest that lipids are also present in the uterine lumen and likely play a critical role in embryo development and conceptus elongation [160, 161, 222]. In cattle, several individual phospholipids and glycerolipids are altered between days 12 and 14 of the estrous cycle, and progesterone supplementation increases uterine luminal total lipid content $[153,156]$. In the bovine conceptus, genes regulating primary metabolism and lipid uptake, metabolism, and de novo synthesis are substantially increased during conceptus elongation [157]. These results emphasize the need to better understand lipids and other metabolites in the uterine environment and their use by the conceptus in the context of whole conceptus metabolism. Here, we present a comprehensive examination of both the lipidome and primary metabolome of the uterine lumen and endometrium of day 14 cyclic and pregnant sheep. 


\section{Materials and Methods}

\section{Animals}

All experimental and surgical procedures for the mature sheep (Ovis aries) were approved by the Institutional Animal Care and Use Committee of the University of Missouri. Adult Rambouillet ewes (O. aries) were group housed, fed a total mixed ration ad libitum, and observed for onset of estrus (day 0). A subset of ewes was mated to an intact ram of proven fertility on day 0 . Ewes were euthanized on day 14 of the estrous cycle (14C) or pregnancy (14P) $(n=6$ per status). The entire female reproductive tract was obtained through a mid-ventral incision, and the broad ligament removed with scissors. A clamp was placed on the internal ostium of the cervix, and the oviduct and uterotubal junction was removed. A Tomcat urinary catheter, attached to a syringe containing $10-\mathrm{ml}$ sterile water, was inserted approximately $1 \mathrm{~cm}$ into the uterine horn contralateral to the corpus luteum. Sterile water was slowly infused into the uterine lumen and then gently massaged through the uterine body into and through the ipsilateral horn that was held over a sterile petri dish using a hemostat. The uterine lumen flush (ULF) was collected in a petri dish and the conceptus removed if present and snap-frozen in liquid nitrogen. The ULF was then transferred to a $15-\mathrm{ml}$ tube, clarified by centrifugation $\left(3000 \times g\right.$ for $15 \mathrm{~min}$ at $\left.4^{\circ} \mathrm{C}\right)$, transferred into a clean tube, and volume recorded prior to storage at $-80^{\circ} \mathrm{C}$. Sections $(\sim 0.5 \mathrm{~cm})$ from the mid-portion of the uterine horn ipsilateral to the ovary were placed in optimum cutting temperature (OCT) compound (Sakura Finetek USA, Inc.), frozen over liquid nitrogen vapor, and stored at $-80^{\circ} \mathrm{C}$. The uterus was then opened, and the 
endometrium physically dissected from the myometrium using surgical scissors and flash-frozen in liquid nitrogen.

Lipidomic and metabolomics

Uterine lumen flush (ULF), endometrium, and conceptus samples were analyzed by the West Coast Metabolomics Center at the University of CaliforniaDavis. Lipids and metabolites were extracted using a modified Maytash method [385]. First, an aliquot of $100 \mu \mathrm{l}$ of ULF, $10 \mathrm{mg}$ of endometrium, or $15 \mu \mathrm{l}$ of conceptus tissue was mixed with $225 \mu$ of cold methanol and quality control mixture (containing odd chain and deuterated lipid internal standards [lysoPE (17:1), lysoPC (17:0), PC (12:0/13:0), PE (17:0/17:0), PG(17:0/17:0), sphingosine (d17:1), d7-cholesterol, SM (17:0), C17 ceramide, d3-palmitic acid, MG (17:0/0:0/0:0), DG (18:1/2:0/0:0), DG (12:0/12:0/0:0), and d5-TG (17:0/17:1/17:0)]. Samples were vortexed for $10 \mathrm{~s}$ and $750 \mu \mathrm{l}$ of cold methyl tert-butyl ether (MTBE) containing CE (22:1) (internal standard) were added. Samples were vortexed for $10 \mathrm{~s}$ again and endometrial tissue was ground in the solution. All samples were then mixed for $6 \mathrm{~m}$ at $4^{\circ} \mathrm{C}$ and $188 \mu$ of mass spectrometry-grade water was added to induce phase separation. Samples were vortexed for $20 \mathrm{~s}$ and then centrifuged $(14,000 \times$ rcf for $2 \mathrm{~m})$. The upper organic phase was collected in a $300 \mu \mathrm{l}$ aliquot and evaporated by SpeedVac. Dried extracts were resuspended in $60 \mu \mathrm{l}$ mixture of a methanol/toluene $(9: 1, \mathrm{v} / \mathrm{v})$ solution containing an internal standard 12[(cyclohexylamino)carbonyl]amino]-dodecanoic acid (CUDA) for quality control.

For lipidomic analysis, $1.7 \mu \mathrm{l}$ and $5 \mu \mathrm{l}$ of each sample was injected for positive and negative ion mode, respectively, and separated on an Acquity UPLC 
CSH C18 column (100 x $2.1 \mathrm{~mm} ; 1.7 \mu \mathrm{m})$. The mobile phase consisted of $60: 40$ acetonitrile:water with $10 \mathrm{mM}$ ammonium formate and $0.1 \%$ formic acid. Samples were run in accordance to the following gradient: 0 min $85 \%(A) ; 0-2$ min $70 \%(A)$; $2-2.5 \min 52 \%(A) ; 2.5-11 \min 18 \%(A) ; 11-11.5 \min 1 \%(A) ; 11.5-12 \min 1 \%(A)$; $12-12.1 \min 85 \%(A)$; and $12.1-15 \min 85 \%(A)$. Liquid chromatography (LC) and quadropole time-of-flight (QTOF) mass spectrometry (MS) were performed on an Agilent 1920 Infinity LC system (G4220A binary pump, G4266A autosampler, and G1316C Column Thermostat) coupled to an Agilent 6530 MS for positive ion mode or Agilent 6550 MS equipped with an ion funnel (iFunnel) for negative mode. The MS scan in the mass range $m / z 65-1700$ producing unique and complementary spectra. A reference solution generating ions of 121.050 and $922.007 \mathrm{~m} / \mathrm{z}$ in positive mode and 119.036 and $966.0007 \mathrm{~m} / \mathrm{z}$ in negative mode was used for continuous mass correction. For data processing, MassHunter software was used to assign a unique ID to each lipid based on its retention time and exact mass (RT_mz). Lipids are identified based on their unique MS/MS fragmentation patterns using in-house software, Lipidblast, and were quantified to class-specific internal standards.

For primary metabolomics, $0.5 \mu$ of each sample was injected with a Gerstel CIS cold injection system. Samples were run on a $30 \mathrm{~m}$ long, $0.25 \mathrm{~mm}$ i.d. Rtx-5Sil MS column $(0.25 \mu \mathrm{m}$ 95\% dimethyl $5 \%$ diphenyl polysiloxane film) with a $10 \mathrm{~m}$ integrated guard column with a mobile helium phase. The oven temperature was held constant at $50^{\circ} \mathrm{C}$ for 1 min and then ramped by $20^{\circ} \mathrm{C}$ per min to $330^{\circ} \mathrm{C}$. Samples were run on an Agilent 6890 gas chromatographer (GC) equipped with a 
Gerstel automatic linear exchange system (ALEX). Samples were then run on the Leco Pegasusus IV TOF MS. The MS was controlled by Leco ChromaTOF software vs. 2.32 (St. Joseph, MI) reading electron impact ionization at $70 \mathrm{~V}$ with a scan mass range of 85-500 Da. Apex masses were reported for use in the BinBase algorithm which uses validity of chromatogram, unbiased retention index marker detection, and retention index calculation by $5^{\text {th }}$ order polynomial regression. Spectra were cut to $5 \%$ base peak abundance and matched to database entries using matching retention index, unique ions and apex masses, and mass spectra similarity. Data were filtered on peak purity and signal/noise ratios. Quantification is reported as peak height. Raw results were normalized by vector normalization. Oil Red O (ORO) staining and quantification

Uteri frozen in OCT compound were cryosectioned at $10 \mu \mathrm{m}$ on a model CM1950 cryostat (Leica Microsystems), thaw mounted on slides, and stored at $20^{\circ} \mathrm{C}$ until staining. Lipid droplets were stained as described [386]. Briefly, ORO stock solution was made by adding $2.5 \mathrm{~g}$ of ORO (Sigma-Aldrich, St. Louis MO) to 99\% isopropyl alcohol (Sigma-Aldrich) and mixed for $2 \mathrm{~h}$ at room temperature. For staining, ORO stock was mixed at a 1.5:1 ratio with distilled water to make a fresh ORO working solution. After $10 \mathrm{~min}$ at $4^{\circ} \mathrm{C}$, the $\mathrm{ORO}$ working solution was filtered $(40 \mu \mathrm{m})$. Frozen sections were equilibrated for $10 \mathrm{~min}$ at room temperature and submerged in working ORO solution for $10 \mathrm{~min}$. Sections were rinsed in running tap water for 30 minutes, and coverslips affixed with Flouromount-G Mounting medium (ThermoFisher Scientific). Brightfield images were collected with a Leica DM5500 B upright microscope using Leica Application Suite X (LAS X) within $6 \mathrm{~h}$ 
of staining. To quantify staining, images were analyzed using ImageJ software $[387,388]$. Briefly, the image was converted to an 8-bit grayscale format and the stained lipid droplets were segmented using thresholding. Threshold conditions were kept the same across all uterine sections. The area marked using the threshold conditions was calculated as a percentage of the total image. The average area covered was averaged between 14C $(n=3)$ and 14P $(n=3)$ ewes. Statistical analysis

Lipid concentrations were summed to quantify each class (e.g. triglycerides, ceramides) for each individual ewe and were subjected to pairwise comparisons to calculate overall trends by lipid class. Statistical analyses were performed using MetaboAnalyst software. A table matrix of individual lipids was uploaded by class and normalized by autoscaling. Volcano plot analysis (fold change threshold 1.5, $P$ value $=0.05)$, t-tests $($ FDR $P$-value $=0.05)$, and heatmaps were used to interrogate the data. Metabolites were also analyzed using MetaboAnalyst software. All quantitative data were subjected to least-squares ANOVA using the GLM procedures of the SAS (SAS Institute Inc., Cary, NC). In all analyses, error terms used in significance were identified according to the expectation of the mean squares for error. Significance $(P \leq 0.05)$ was determined by the probability differences of the least-squares means.

\section{Results}

\section{Lipidomics}


In total, 406 individual lipids, spanning 16 classes, were identified and quantified in the endometrium, ULF, and/or conceptus (Supplementary Table S1). The endometrium lipidome was largely unaffected by pregnancy. Total triglycerides (TAG) did not differ $(P=0.08)$ between the endometrium of day 14 cyclic (14C) and pregnant (14P) ewes (Figure 1A). Eighteen (18) of the 117 individually measured TAG were differentially abundant in the endometrium (Figure 1B). ORO staining revealed that lipid droplets were present almost exclusively in the cells of the luminal epithelium (LE) and superficial glandular epithelium (GE) of the endometrium (Figure 1C). Of note, lipid droplets were less abundant in the endometrial epithelium of 14P sheep (Figure 1D).

Total TAGs in ULF was not different $(P=0.08)$ (Figure 2A), but 51 individual TAG were elevated in the ULF of 14P sheep (Figure 2B). Heatmap analysis of TAG in the ULF shows that ewes clustered by pregnancy status (Figure 2C). Total phosphatidylcholine (PC), phosphatidylinositol (PI), phosphatidylglycerol (PGL), and diacylglycerol (DG) phospholipids were more abundant in the uterine lumen of $14 \mathrm{P}$ sheep than $14 \mathrm{C}$ sheep (Figure 3 ). Total ceramides were higher in the uterine lumen of 14P ewes. Non-esterified fatty acids (NEFA) were not different; however, 5 individual ceramides and 2 individual NEFA were elevated in the uterine lumen of 14P ewes (Table 1). Cholesteryl esters were lower in the uterine lumen of $14 \mathrm{P}$ ewes, while cholesterol was not different between $14 \mathrm{C}$ and $14 \mathrm{P}$ ewes. Several other lipid classes, including phosphatidylethanolamine (PE) and phosphatidylserine (PS) phospholipids, acylcarnitines and sphingolipids, did not differ in the uterine lumen of $14 \mathrm{C}$ or $14 \mathrm{P}$ sheep. 
A

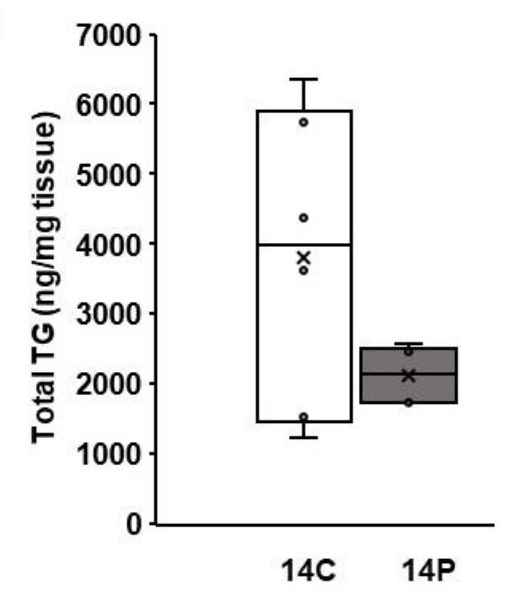

C

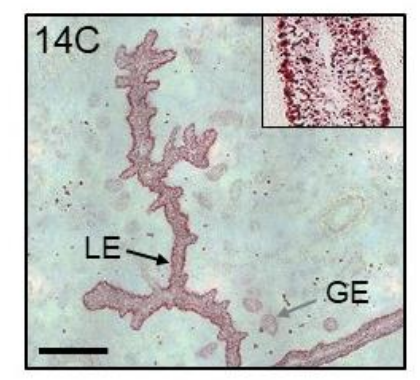

B



D

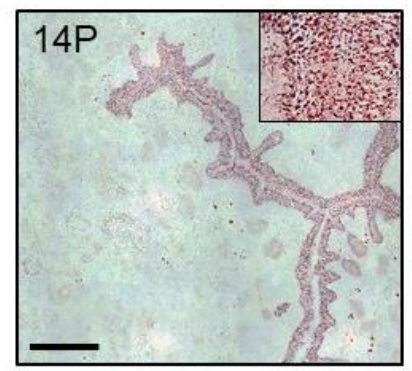



Figure 1. Triglycerides (TAG) in the endometrium from day 14 cyclic and pregnant sheep. (A) Endometrial TAG were not different $(P=0.08)$ between day 14 cyclic (14C) and pregnant (14P) sheep. (B) Volcano plot analysis showing 21 individual endometrial TAG lower in 14P sheep, including triglyceride (TAG) 53:5 and 46:0. (C) Uterine sections stained with Oil Red $O$ stain. Inlaid images of LE at 20x magnification. Scale bar: $400 \mu \mathrm{m}$. (D) Lipid droplets more abundant $(\mathrm{P}<0.01)$ in the endometrial epithelium of $14 \mathrm{C}$ sheep. 
A

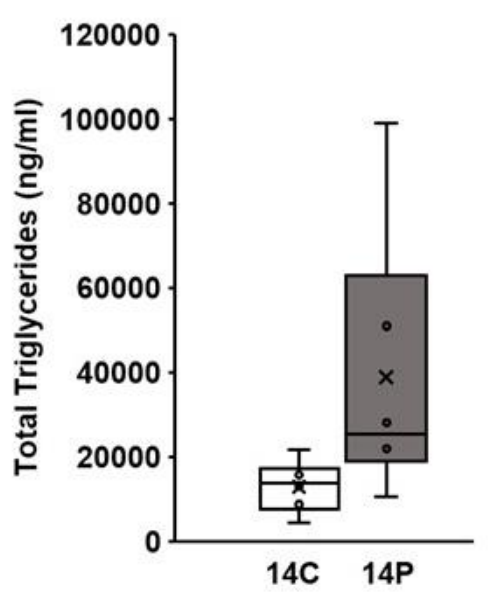

B



C



Figure 2. Triglycerides (TAG) in the uterine lumen. Lipidomic analysis was performed on the uterine lumen flush (ULF) of day 14 cyclic (14C) and pregnant (14P) sheep. (A) TAG were not different $(P=0.08)$ between $14 \mathrm{C}$ and 14P sheep. (B) Volcano plot analysis found that 51 individual TAG, including 53:5 and 46:0, were more abundant in the uterine lumen of pregnant sheep. (C) Heatmap analysis of TAG in the ULF of 14C (red) and 14P (blue) sheep. 

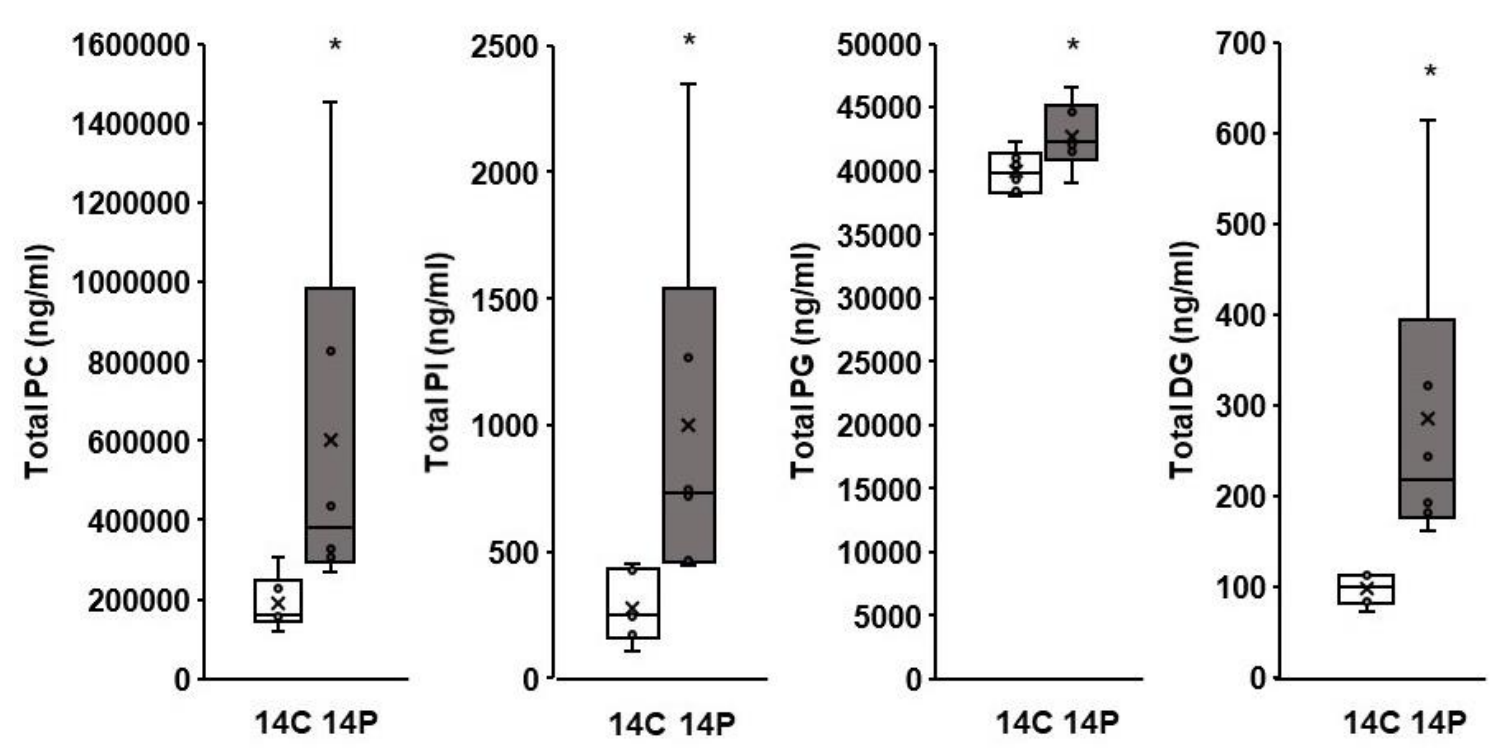

Figure 3. Phospholipids in the uterine lumen. Total phosphatidylcholine $(\mathrm{ng} / \mathrm{ml})$, phosphatidylinositol, phosphatidylglycerol, and diacylglycerols were elevated in the uterine lumen of day 14 pregnant (14P) as compared to cyclic (14C) sheep. The asterisk denotes a difference $(P \leq 0.05)$.

To discern lipid populations in the conceptus, individual lipids were summed by class and calculated as a proportion of the sum of total lipids (by weight) in parallel to the uterine lumen (ULF) and endometrium (Table 2). Notably, $44.4 \%$ of total conceptus lipids were TAG, whereas total endometrial lipids were only $4.1 \%$ in the endometrium and $0.8 \%$ in the ULF. In the ULF and endometrium, $70 \%$ and $95 \%$ of the total lipid weight was phospholipids, while only $54 \%$ of the conceptus lipid weight was phospholipids. As a proportion of total lipid makeup, acylcarnitines were 12.5-fold lower as a proportion of total lipid weight in the conceptus $(0.0004 \%)$ compared to the endometrium $(0.005 \%)$ and the ULF $(0.005 \%)$. 
Table 1. Individual ceramide and NEFA lipids altered in the uterine lumen of day 14 cyclic (C) and pregnant $(P)$ sheep.

\begin{tabular}{lccc}
\hline & $\begin{array}{c}\text { 14C } \\
\text { (Total ng) }\end{array}$ & $\begin{array}{c}\text { 14P } \\
\text { (Total ng) }\end{array}$ & FDR P-Value \\
\hline Ceramides & & & \\
\hline Ceramide (d34:2) & $15.5 \pm 3.6$ & $45 \pm 7.0$ & 0.023 \\
Ceramide (d41:1) & $49.2 \pm 2.4$ & $61.3 \pm 5.5$ & 0.032 \\
Ceramide (d42:0) & $19.5 \pm 1.2$ & $30.7 \pm 3.0$ & 0.032 \\
Ceramide (d42:1) & $110.5 \pm 16.1$ & $286.5 \pm 38.1$ & 0.023 \\
Ceramide (d42:2) & $6.5 \pm 3.1$ & $24.2 \pm 8.0$ & 0.006 \\
\hline Non-esterified Fatty Acids (NEFA) & & \\
\hline Eicosadienoic acid (20:2) & $22.2 \pm 2.5$ & $48.2 \pm 5.8$ & 0.022 \\
Linolenic acid (18:3) & $43.4 \pm 8.1$ & $163.5 \pm 28.4$ & 0.022 \\
\hline
\end{tabular}

Table 2. Total lipids in the conceptus, endometrium and uterine lumen on day 14 of pregnancy in sheep 1 .

\begin{tabular}{lccc}
\hline & Conceptus & Endometrium & Lumen (ULF) \\
\hline Acylcarnitines & $0.0004 \pm 0.000 \%$ & $0.005 \pm 0.000 \%$ & $0.005 \pm 0.001 \%$ \\
Cholesteryl esters & $0.05 \pm 0.00 \%$ & $0.09 \pm 0.01 \%$ & $19.34 \pm 2.34 \%$ \\
Ceramides & $0.06 \pm 0.01 \%$ & $0.02 \pm 0.00 \%$ & $0.14 \pm 0.02 \%$ \\
Cholesterol & $0.41 \pm 0.01 \%$ & $0.96 \pm 0.04 \%$ & $1.44 \pm 0.19 \%$ \\
Diglycerides & $0.02 \pm 0.00 \%$ & $0.02 \pm 0.00 \%$ & $0.03 \pm 0.00 \%$ \\
Ether-linked phospholipids & $0.14 \pm 0.01 \%$ & $0.53 \pm 0.02 \%$ & $0.25 \pm 0.02 \%$ \\
Glucosyl/Hexosyl ceramides & $0.01 \pm 0.00 \%$ & $0.002 \pm 0.00 \%$ & $0.09 \pm 0.02 \%$ \\
Lysophospholipids & $0.01 \pm 0.00 \%$ & $0.01 \pm 0.00 \%$ & $0.07 \pm 0.00 \%$ \\
Non-esterified fatty acids & $0.02 \pm 0.00 \%$ & $0.02 \pm 0.00 \%$ & $0.26 \pm 0.02 \%$ \\
Phosphatidylcholines & $52.23 \pm 3.52 \%$ & $93.32 \pm 0.39 \%$ & $60.84 \pm 3.70 \%$ \\
Phosphatidylethanolamines & $1.70 \pm 0.08 \%$ & $2.62 \pm 0.10 \%$ & $2.39 \pm 0.18 \%$ \\
Phosphatidylglycerides & $0.40 \pm 0.02 \%$ & $0.76 \pm 0.09 \%$ & $9.10 \pm 0.99 \%$ \\
Phosphatidylinositol & $0.12 \pm 0.02 \%$ & $0.05 \pm 0.01 \%$ & $0.10 \pm 0.01 \%$ \\
Phosphatidylserine & $0.04 \pm 0.00 \%$ & $0.20 \pm 0.01 \%$ & $0.18 \pm 0.03 \%$ \\
Sphingomyelin & $0.42 \pm 0.02 \%$ & $0.56 \pm 0.05 \%$ & $1.63 \pm 0.17 \%$ \\
Triglycerides & $44.39 \pm 3.57 \%$ & $0.83 \pm 0.15 \%$ & $4.15 \pm 0.45 \%$ \\
\hline
\end{tabular}

${ }^{1}$ Wet weight for individual lipids of specific subclasses were summed together and calculated as a percentage of total wet weight to understand the makeup of the conceptus, endometrium, and uterine lumen (ULF). 
Principal component analysis (PCA) of the entire lipidome across all three sample types revealed clear separation of the conceptus from the endometrium and the ULF, which clustered closely together (Figure 4). Of note, several individual lipid species were not present across all three sample types. For example, PC (p-38:3)/PC (o-38:4) was exclusive to the endometrium, whereas PC (31:1) was identified in the endometrium and conceptus. Moreover, SM (d38:2) and TAG (55:0) was mostly absent from the endometrium and conceptus but was present in the uterine lumen (Table S1).

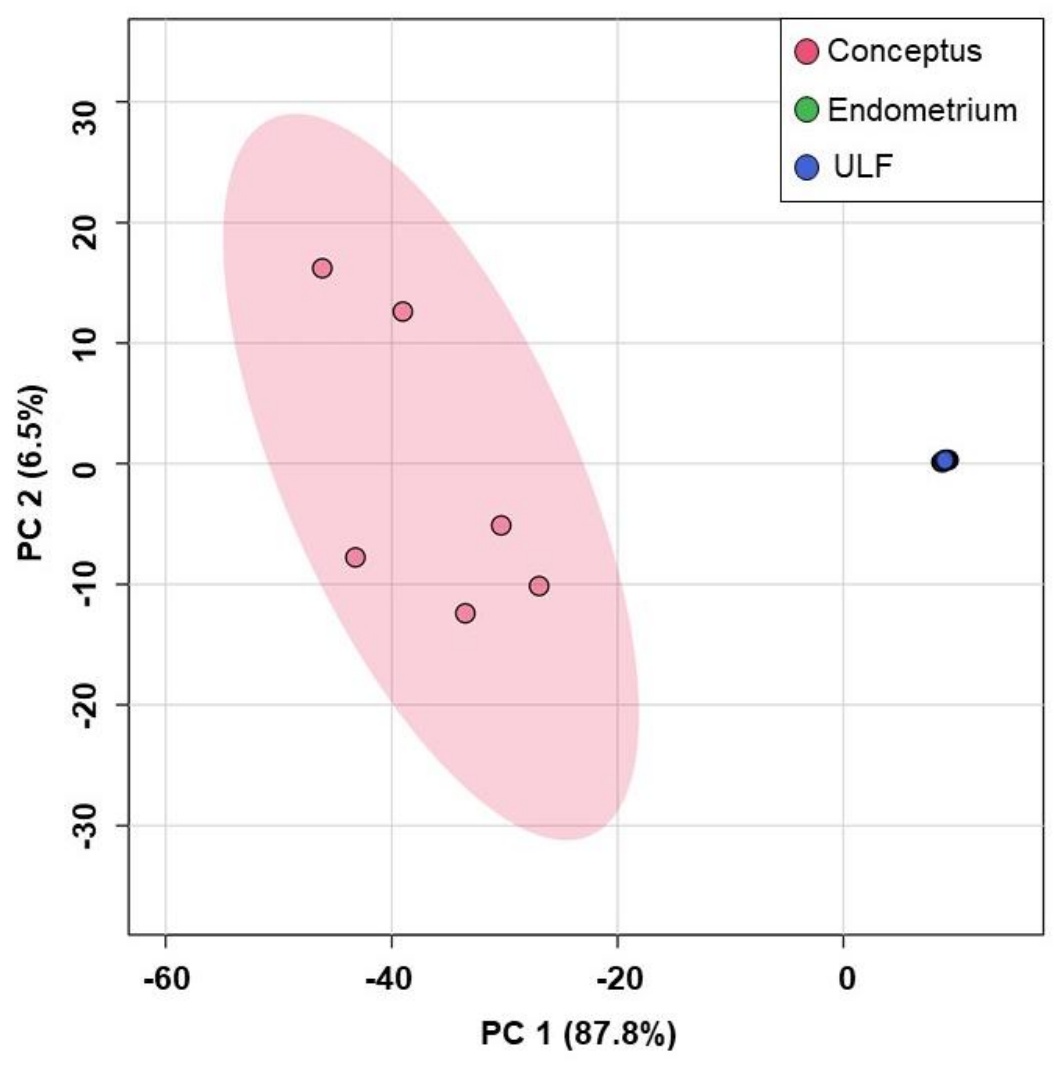

Figure 4. Principal component analysis (PCA) of the conceptus, endometrial, and uterine lumen flush (ULF) lipidome. Note the distinct separation of the day 14 conceptus from endometrium and ULF. 


\section{Metabolomics}

Primary metabolites in the endometrium, ULF, and conceptus are provided in Table S2. Metabolites that did not fulfill high quality criteria for identification and quantification were considered absent from the sample type. Of the 211 different

metabolites measured, 198 metabolites were present in the endometrium and 172 in the conceptus. There were no pregnancy-associated changes in endometrial metabolites.

In the ULF, 152 metabolites were identified with high confidence, and PCA revealed only a modest separation in the ULF samples of $14 \mathrm{C}$ and 14P sheep (Figure 5A). In total, 36 metabolites were altered by pregnancy status (Figure 5B) of which 13 and 23 were lower and higher in the ULF of 14P ewes, respectively (Tables 3 and 4). Metabolite enrichment analysis of the metabolites elevated in ULF of $14 \mathrm{P}$ ewes highlighted: ammonia recycling, urea cycle, amino acid metabolism, purine metabolism, gluconeogenesis, and the Warburg effect (Figure 5C). Of the proteinogenic amino acids, 14 were elevated in the uterine lumen of 14P ewes, while only one, leucine, was lower $(P<0.05)$ (Figure 6). PCA analysis of the primary metabolome showed clear separation of the conceptus, endometrium, and the ULF (Figure 7). 
A

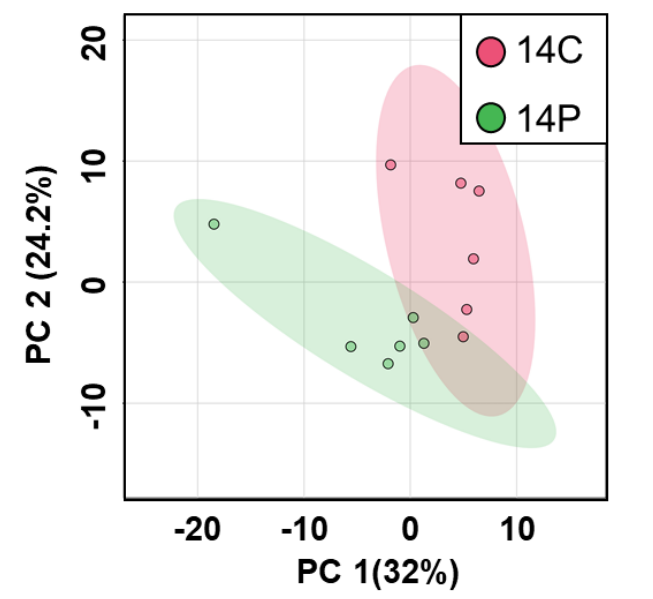

B

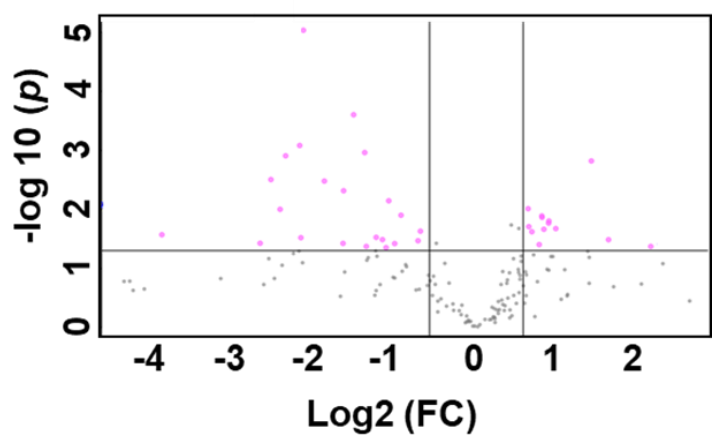

C

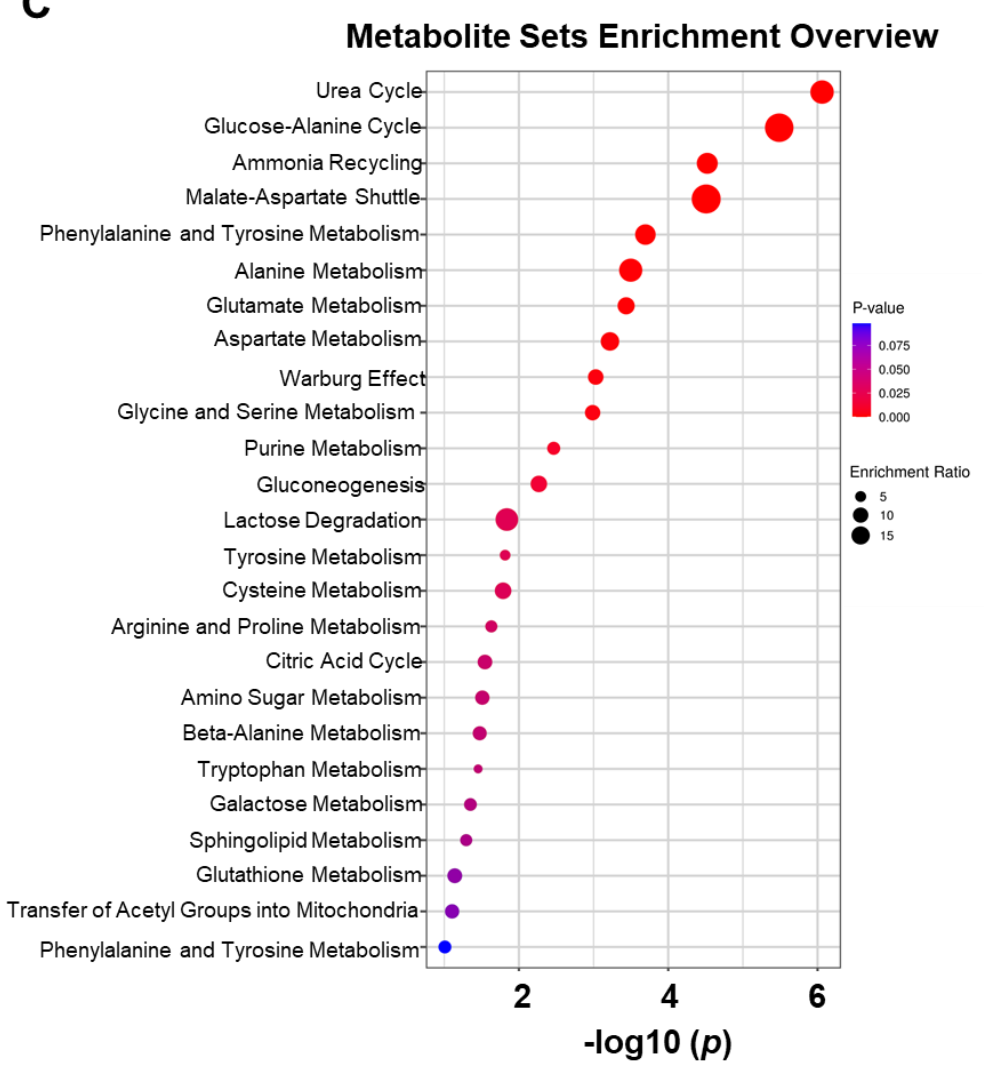

Figure 5. Enrichment analysis of metabolites elevated in the uterine lumen of day 14 cyclic (14C) and pregnant (14P) sheep. (A) Principal component analysis modestly separated day 14 cyclic and pregnant sheep by their primary metabolome. (B) Volcano plot analysis found 57 metabolites differentially abundant between cyclic and pregnant sheep. (C) Metabolites that were more abundant in the uterine lumen of pregnant sheep were entered into MetaboAnalyst Enrichment Analysis. 
Table 3. Top 10 metabolites more abundant in the uterine lumen of day 14 pregnant (P) than cyclic (C) sheep ${ }^{1}$.

\begin{tabular}{ccccc}
\hline Metabolites & 14C & 14P & Fold Change & P-Value \\
\hline a-ketoglutarate & $298.2 \pm 74.7$ & $4603.6 \pm 1621.9$ & 15.4 & 0.02 \\
Pyruvic acid & $591 \pm 263.5$ & $3942.3 \pm 1334.2$ & 6.7 & 0.03 \\
Glutamine & $9773.6 \pm 2070.0$ & $58074.3 \pm 11824.3$ & 5.9 & $<0.01$ \\
Phenylalanine & $2950.9 \pm 686.6$ & $16381.4 \pm 4045.2$ & 5.5 & $<0.01$ \\
Glutamic acid & $23076.7 \pm 4031.3$ & $122348.0 \pm 22114.3$ & 5.3 & $<0.01$ \\
Threonine & $6940.1 \pm 1613.5$ & $32385.8 \pm 4857.5$ & 4.6 & $<0.01$ \\
Aspartic acid & $6637.4 \pm 1762.5$ & $30783.8 \pm 9102.0$ & 4.6 & 0.03 \\
Serine & $76362.0 \pm 17510.4$ & $342596.3 \pm 22584.0$ & 4.5 & $<0.01$ \\
Tryptophan & $2769.6 \pm 798.9$ & $10457.8 \pm 1752.3$ & 3.7 & $<0.01$ \\
Pseudouridine & $609.5 \pm 44.8$ & $1944.7 \pm 539.2$ & 3.2 & 0.04 \\
\hline
\end{tabular}

${ }^{1}$ Values are presented as mean \pm SEM

Table 4. Top 10 metabolites less abundant in the uterine lumen of day 14 pregnant $(P)$ than cyclic $(C)$ sheep 1 .

\begin{tabular}{ccccc}
\hline Metabolite & $\mathbf{1 4 C}$ & $\mathbf{1 4 P}$ & Fold Change & P-Value \\
\hline Cyclohexylamine & $924.6 \pm 112.3$ & $529.4 \pm 70.0$ & 0.6 & 0.013 \\
$\begin{array}{c}\text { Dehydroabietic } \\
\text { acid }\end{array}$ & $1729.3 \pm 230.2$ & $987.7 \pm 91.1$ & 0.6 & 0.014 \\
Parabanic acid & $2739.8 \pm 430.8$ & $1542.1 \pm 114.6$ & 0.6 & 0.022 \\
Hydroxylamine & $551897.0 \pm 80743.2$ & $297361 \pm 49869.1$ & 0.5 & 0.016 \\
Capric acid & $767.7 \pm 106.1$ & $414.8 \pm 36.8$ & 0.5 & 0.016 \\
Benzoic acid & $7043.1 \pm 1346.9$ & $3569.2 \pm 373.3$ & 0.5 & 0.022 \\
4-hydroxybutyric & $436.1 \pm 133.0$ & $164.9 \pm 11.7$ & 0.4 & 0.039 \\
acid & $564701.0 \pm 64348.6$ & $211604.0 \pm 56483.8$ & 0.4 & 0.001 \\
Leucine & $575.3 \pm 164.2$ & $183.0 \pm 28.8$ & 0.3 & 0.036 \\
Ribonic acid & $2276.9 \pm 757.5$ & $516.0 \pm 51.8$ & 0.2 & 0.036 \\
Adenosine & &
\end{tabular}

${ }^{1}$ Values are presented as mean \pm SEM 




Figure 6. Amino acids in the uterine lumen. Amino acids were largely elevated in the uterine lumen of pregnant sheep. The asterisk denotes a difference ( $P \leq$ 0.05).

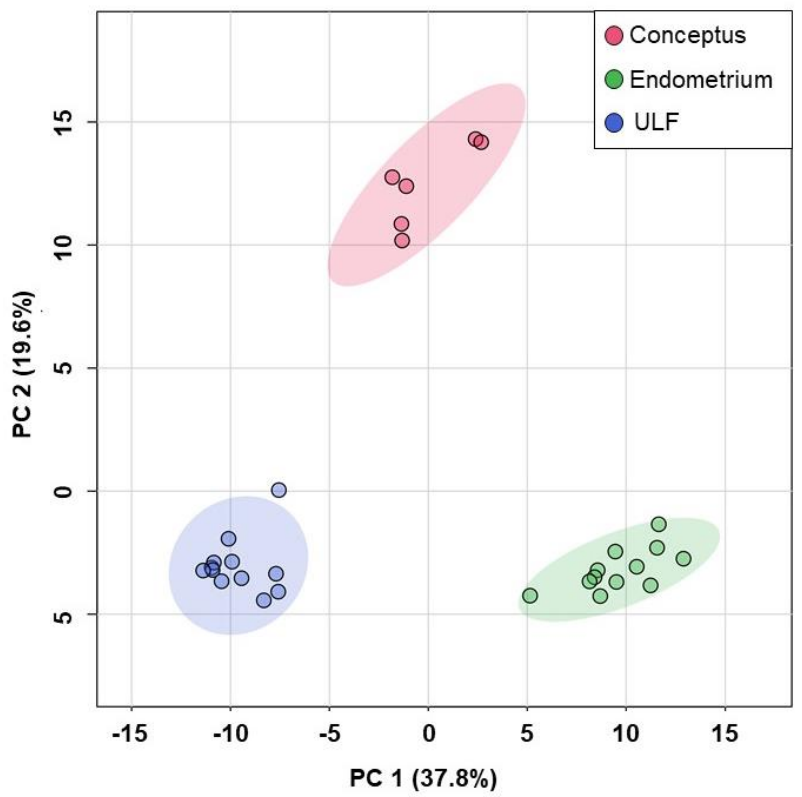

Figure 7. Principal component analysis of the day 14 sheep conceptus, endometrium, and uterine lumen (ULF) metabolome. Note the distinct separation of the day 14 conceptus, endometrium and ULF samples from each other. 


\section{Discussion}

Dynamic changes in the uterine metabolome occur during early pregnancy in sheep and cattle $[138,153,154,156,364,389]$. These data support the hypothesis that lipids and primary metabolites are an integral component of endometrial secretions or uterine histotroph and are likely important substrates for cellular growth and development during conceptus elongation. Available data also support the idea that the endometrium selectively mobilizes nutrients and other biomolecules into the uterine lumen for use by the developing conceptus during this crucial period of early pregnancy susceptible to high pregnancy loss $[138,139$, $145,300]$.

The endometrium must maintain homeostasis during the estrous cycle and early pregnancy; thus, it was expected that the endometrial lipidome would be relatively unaltered by pregnancy $[390,391]$. Indeed, endometrial TAG were the only lipid class affected by pregnancy, as several individual TAG were lower in 14P ewes than 14C sheep. Thus, TAG likely play important roles in early pregnancy. Intriguingly, several studies have reported that lipid droplets accumulate in the endometrial LE across the estrous cycle and are depleted in pregnancy, particularly by day $14[162,163]$. Lipid droplets are cellular organelles that store and hydrolyze TAG and other neutral lipids, and likely account for the results observed here [392, 393]. In the present study, lipid droplets were present almost exclusively in the cells of the LE, where they notably accumulated on the basolateral side of the epithelial cells in $14 \mathrm{C}$ sheep and were spread throughout 
the epithelial cells in 14P sheep. As the whole endometrium was analyzed, it is possible that there are differences in the lipid populations of specific subsets of endometrial cells that could not be captured using this approach. Alternative technologies, such as in situ MALDI imaging of uterine lipids could enable more detailed examinations of specific subsets of cell types to clarify this [394, 395].

In contrast to the endometrium, there were many pregnancy-associated differences in specific lipid species present in the uterine lumen. While total TAG amounts were not different, $51(43 \%)$ of the individual TAG were elevated in the ULF of 14P sheep. Interestingly, 8 of these TAG were correspondingly decreased in the endometrium of 14P sheep. Furthermore, the top 10 most abundant TAG in the ULF and endometrium were almost identical, suggesting that the origin of ULF TAG is the endometrium. These 8 altered TAG represent potentially important targets to better understand maternal regulation of conceptus elongation by lipids. Total phospholipid (PC, PI, PGL, and DG) levels were elevated in the uterine lumen of 14P sheep. Given that phospholipids are core components of cellular and organelle membranes, there is likely a large demand for phospholipid substrate by the conceptus [19]. Future studies could focus on understanding why specific phospholipid populations are different in the uterine lumen of 14C and 14P sheep. For example, PE, which is the second most common phospholipid in most cell membranes after PC, was not different between 14C and 14P sheep while PI was [396]. Although PI is less common in membranes than PE, it plays important roles in cell signaling as a second messenger and thus it is possible that it is specifically mobilized for the conceptus [397, 398]. In fact, mice with a global deletion in PI 
transfer protein beta (PITPNB) exhibit embryo lethality, indicating that PI phospholipids are an important phospholipid species in the developing embryo [399].

Since TAG and phospholipids are hydrophobic and mobilized to the uterine lumen, future studies should clarify how these lipid populations are transported. One possible mechanism could be the secretion of endometrial lipid droplets into the uterine lumen. In mammary gland epithelial cells, lipid droplets are synthesized on the endoplasmic reticulum and accumulate on the basolateral side, like they seemingly do in the endometrial epithelial cells [400]. They are subsequently secreted from the apical side to rapidly mobilize lipids into milk [401]. Lipid droplets could be mobilized from the endometrium directly into the uterine lumen in a similar manner, as many studies have characterized lipid droplet accumulation during the estrous cycle and their depletion in pregnant sheep [162, 163, 402]. Perhaps most convincingly, various perilipin proteins (PLIN1, PLIN2, PLIN3), which are specific markers of lipid droplets, are present in the ULF of cattle during conceptus elongation [74, 403].

An alternative means to transport TAG and phospholipids is via packaging in lipoproteins [234, 404, 405]. In lipoproteins, apolipoproteins and phospholipids surround neutral cores of TAG and CEs. Of note, the ovine conceptus expresses genes encoding various apolipoproteins (e.g. APOA1 and $A P O E$ ) which are upregulated during conceptus elongation [72]. Further, apolipoprotein AI (APOA1) is more abundant in the uterine lumen of pregnant compared to cyclic sheep [73]. The presence of albumin in ULF suggests that proteins from serum are able to 
cross into the uterine lumen, and lipoproteins, which are also abundant in the blood, could enter the uterus this way as well $[73,406,407]$. Consistent with this, two individual lipids [SM (d38:2) and TAG (55:0)] were absent from the endometrium and conceptus but present in ULF, suggesting that some uterine lumenal lipids are indeed externally derived. Finally, the presence of phospholipids could be due to extracellular vesicles in the uterine lumen [364].

Several individual ceramides and NEFA were also elevated in the uterine lumen of $14 \mathrm{P}$ sheep. Ceramides are lipids that can be incorporated into the sphingolipid portion of cell membranes or independently have roles in cell signaling pathways. Ceramides play critical roles in regulating cell differentiation as well as cell proliferation, and it is quite possible that these ceramides might regulate cell proliferation in the developing conceptus, and would be interesting lipids to further investigate [351, 364, 408]. Principal component analysis of the lipidome across all three sample types found that the ULF and endometrium have similar lipid makeups which differ to that of the conceptus. Thus, the conceptus is unlikely to passively uptake lipids from the ULF, but selectively internalizes specific lipid species or lipid macromolecules. For example, TAG are the most abundant type of lipid in the conceptus, unlike the endometrium and ULF. This observation supports the hypothesis that TAG might be a primary mode of lipid transport to the conceptus. While there is evidence that bovine embryos use TAG as an energy source in vitro, acylcarnitines are only a very small component of total lipids in the conceptus [409]. Acylcarnitines are indicative of active lipid transport across the mitochondrial membrane for beta oxidation, which suggests that the conceptus is 
likely not actively oxidizing lipids for energy production [410]. Thus, TAG are unlikely a major metabolic substrate for the conceptus, but rather may provide a more structural or functional role during conceptus elongation.

Primary metabolite analysis was also performed on the endometrium, ULF, and conceptus. Similar to the lipidome, the endometrial metabolome was almost identical in $14 \mathrm{C}$ and $14 \mathrm{P}$ sheep. In contrast to the endometrium, there were many differences in the primary metabolome in the uterine lumen of $14 \mathrm{C}$ and $14 \mathrm{P}$ sheep. Most strikingly, alpha-ketoglutarate was 15.4 -fold more abundant in the uterine lumen of $14 \mathrm{P}$ versus $14 \mathrm{C}$ sheep. In cattle, alpha-ketoglutarate decreased between days 12 and 14 of the estrous cycle [147]. Future work should clarify whether it is elevated in $14 \mathrm{P}$ ewes due to production by the conceptus or the underlying pregnant endometrium. Additionally, understanding conceptus turnover of this metabolite could help clarify its role in early conceptus development; alphaketoglutarate is a rate-determining intermediate in the TCA cycle but also has key roles in amino acid synthesis, notably through its interconversion to glutamine and as an enhancer of protein synthesis [411, 412].

Consistent with many previous reports, several amino acids were elevated in the uterine lumen of $14 \mathrm{P}$ sheep $[138,145,179]$. Histidine, glutamine, and lysine displayed the largest pregnancy-dependent increases in the ULF. Both histidine and lysine are essential amino acids that cannot be synthesized de novo, highlighting the importance of uterine transportation of these amino acids to the conceptus $[138,145,150,413]$. While glutamine is nonessential, highly proliferating cells rely on glutamine, likely due to its role as a nitrogen donor for 
amino acid and nucleotide synthesis, emphasizing its importance to support conceptus elongation [414]. Glutamine also activates components of the mammalian target of rapamycin (MTOR) signaling pathway in the ovine conceptus and increase its ability to translate proteins $[415,416]$. Of all the amino acids, leucine, also an essential amino acid, was the only amino acid lower in the uterine lumen of 14P sheep. Previous reports found that leucine was higher in the ULF of pregnant sheep versus cyclic sheep on days 15 and 16 [138]. As this study was conducted on day 14 , it is possible that the results here are indicative of temporal changes in amino acid turnover by the conceptus that were not captured in previous studies, and that uterine mobilization of leucine specifically increases between days 14 and 15 of pregnancy.

Ribonucleosides, which make up RNA molecules, are present in the uterine lumen of cattle and increase in uterine abundance between days 12 and 14 of the estrous cycle [147]. Three of the four most common ribonucleosides (adenosine, guanosine, and uridine) were identified in ovine ULF here and, of these, adenosine and guanosine, as well as pseudouridine, a modified uridine, were lower in the uterine lumen of $14 \mathrm{P}$ sheep. Extracellular vesicles (EV) are in the uterine lumen during early pregnancy and contain cargo including nucleic acids in the form of RNA and miRNA. The change in ribonucleosides may be due to the presence of nucleic acids in ULF EV. Indeed, EVs increase during the estrous cycle but are notably lower in the ULF of pregnant sheep on days 12 and 14 of pregnancy. Thus, it is possible that findings here are due to conceptus uptake of EV that, in turn, affect trophectoderm proliferation and protein translation [120, 364]. 
To better understand the overall implications of the changing ULF metabolome, metabolites that were elevated in the uterine lumen of 14P sheep were analyzed by MetaboAnalyst Enrichment analysis [417, 418]. Two of the most enriched metabolic pathways in the uterine lumen were ammonia recycling and urea cycle. In the developing conceptus, amino acid metabolism is active and activates MTOR signaling to regulate cell proliferation [419]. Ammonia recycling and the urea cycle remove or transform ammonia, a metabolic waste product from central amino acid metabolism, into urea or other products to maximize nitrogen utilization [420]. Several studies found that production of ammonia and lactic acid by the conceptus and its accumulation in the uterus may alter the $\mathrm{pH}$ and physiology of the uterine environment [421, 422]. Future work could determine how the uterus responds to this increased production of metabolic byproducts by the conceptus, how they may regulate conceptus elongation, and if there are active clearance mechanisms by the uterus.

Another hallmark of early embryo metabolism is its heavy reliance on glucose metabolism and the conversion of pyruvate to lactic acid [164, 171]. Gluconeogenesis, the pathway used to synthesize glucose from precursor molecules such as lactate and amino acids, was enriched, suggesting the conceptus may be actively synthesizing glucose. The malate-aspartate shuttle, which translocates electrons produced during glycolysis, and the Warburg effect, which favors fermentation of glycolytic products over their use in aerobic respiration, were also enriched, and highlight the use of these metabolic strategies for energy production during conceptus elongation [164, 423]. Additionally, 
glucose, as well as other carbohydrates which can be used to generate glucose, such as galactose and maltotriose, were elevated in the uterine lumen of $14 \mathrm{P}$ sheep in the present study $[424,425]$. Overall, the primary metabolome of the ULF differs between 14C and 14P sheep, likely reflective of the catabolic, anabolic, and bidirectional flux of metabolites between the conceptus and endometrium.

Moreover, the metabolome of the ULF, endometrium, and conceptus were distinct from each other, as PCA showed clear separation of all three sample types. For example, 52 of metabolites identified with high confidence in the endometrium were not identified within the ULF. Of these, 37, including spermidine, pyrophosphate, and hexose-6-phosphate were identified in the conceptus. Many of these are metabolic pathway intermediates or involved in cellular maintenance, suggesting that the conceptus is actively synthesizing these metabolites rather than obtaining them from the ULF [426-428]. Finally, there were no metabolites identified exclusively in ULF, suggesting that primary metabolites in the ULF likely come from either the conceptus, the endometrium, or both.

Taken together, these results demonstrate that there are dynamic changes in the ULF metabolome and identify several lipids and primary metabolites to investigate for future studies. While the endometrium is metabolically similar between cyclic and pregnant sheep on day 14, it likely plays crucial roles in mobilizing various lipid macromolecules and primary metabolites into the uterine lumen. Enrichment analysis of the ULF and lipid makeup of the conceptus suggest that the conceptus heavily relies on amino acid and glucose metabolism during elongation and readily internalizes TAG, as a structural or functional substrate, 
from the uterine environment. Other lipids, such as phospholipids and ceramides, may provide substrates for cellular and organelle membrane biogenesis during proliferation and play important roles in mediating cell signaling pathways. Overall, these findings support the hypothesis that uterine secretions supply critical substrates and material to support conceptus growth and elongation during a critical period of pregnancy establishment.

\section{Acknowledgements}

We thank David Todd for his expert assistance with sheep husbandry and experiments and Dr. Constantine A. Simintiras for his expert advice and assistance with data analysis, review, and interpretation of results.

\section{Supplemental Information}

Supplemental Table 1. Lipidome of endometrium, ULF, and conceptus.

Supplemental Table 2. Metabolome of endometrium, ULF, and conceptus.

\section{Available upon request or after publication at}

http://www.biolreprod.org/ 


\section{CHAPTER V:}

PROSTAGLANDIN-ENDOPEROXIDE SYNTHASE 2 IS NOT REQUIRED FOR PREIMPLANTATION OVINE CONCEPTUS DEVELOPMENT IN SHEEP

Eleanore V. O'Neil, Kelsey Brooks, Gregory W. Burns, M. Sofia Ortega, Anna C. Denicol, Luis H. Aguiar, Gabriela H. Pedroza, Joshua Benne, Thomas E. Spencer

This work is published in:

Mol Reprod Dev. 2020 Jan;87(1):142-151. doi: 10.1002/mrd.23300. Epub 2019 Nov 20. PMID: 31746519. 


\begin{abstract}
Conceptus elongation is required for successful pregnancy establishment in ruminants and is coincident with the production of interferon tau (IFNT) and prostaglandins (PGs) by the trophectoderm. In both the trophectoderm and endometrium, PGs are primarily synthesized through a prostaglandinendoperoxide synthase 2 (PTGS2) pathway and modify endometrial gene expression and histotrophic makeup to promote conceptus growth and survival. Inhibition of PG production by both the endometrium and the trophectoderm prevented conceptus elongation in sheep. However, the contributions of conceptus-derived PGs to conceptus elongation remains unclear. In this study, CRISPR-Cas9 genome editing was used to inactivate PTGS2 in ovine embryos to determine the role of PTGS2-derived PGs in conceptus elongation. PTGS2 edited conceptuses produced fewer PGs but secreted similar amounts of IFNT to their Cas9 control counterparts and elongated normally. Expression of PTGS1 was lower in PTGS2 edited conceptuses, but PPARG expression and IFNT secretion were unaffected. Content of PGs in the uterine lumen was similar, and gene expression in the endometrium of ewes who received either Cas9 control or PTGS2 edited conceptuses was similar. These results support the idea that conceptus-derived PGs are not required for trophectoderm survival or elongation during the peri-implantation period of pregnancy.
\end{abstract}




\section{Introduction}

In ruminants, placentation is preceded by elongation of the mononuclear trophectoderm of the developing conceptus (embryo and its associated extraembryonic membranes). After entering the uterus as a morula around day 6 (day 0 is estrus and mating), the embryo develops into a blastocyst that hatches from the zona pellucida around day 8 and develops into a spherical conceptus by day 10 . The conceptus continues to undergo marked morphological changes as it grows to an ovoid, tubular, and finally filamentous form reaching greater than 10 $\mathrm{cm}$ in length by day $14[19,28]$. Coincident with elongation, the mononuclear trophectoderm cells synthesize and secrete interferon tau (IFNT), which acts on the endometrium to inhibit the production of luteolytic pulses of PGF2 $\alpha$, maintaining the corpus luteum and progesterone production $[14,356]$. Moreover, conceptus-produced factors, including IFNT, work in concert with progesterone from the corpus luteum to drive changes in endometrial epithelial gene expression $[64,429]$. This molecular crosstalk between the developing trophectoderm of the elongating conceptus and the underlying epithelium of the endometrium regulate conceptus elongation and other early pregnancy events [28, 64, 429].

A hallmark of the peri-implantation period of development in domestic species is the production of large amounts of prostaglandins (PGs), which are biologically active oxygenated fatty acids. The rate limiting step in PG biosynthesis is the conversion of arachidonic acid to prostaglandin $\mathrm{H} 2$ (PGH2) by a PG synthase (PTGS). The dominant PG synthase enzyme in both the ovine endometrium and trophectoderm is prostaglandin-endoperoxide synthase 2 
(PTGS2) $[214,220]$. Indeed, expression of PTGS2 in the conceptus increases more than 20 -fold at the onset of elongation, and conceptuses produce a variety of PGs including PGE2, PGF2 $\alpha$, and PGI2 $[72,221,430]$. The uterine epithelia produce PGs throughout the estrous cycle and especially during pregnancy, as IFNT increases endometrial production of PGs and PTGS2 activity [216, 431, 432].

In sheep, PGs modify endometrial gene expression and histotrophic makeup to promote conceptus growth and survival [50, 433]. When PGs were infused into the uterus at the levels produced by a day 14 ovine conceptus between days 8-14 of a cyclic ewe, expression of various genes, including gastrin-releasing peptide (GRP), insulin like growth factor binding protein 1 (IGFBP1) and galectin15 (LGALS15), was increased in the endometrium [50, 223, 225, 433]. These genes stimulate trophectoderm cell proliferation, migration, and/or attachment and thus directly affect pregnancy success [318, 433, 434]. Brooks et al. [435] demonstrated that PPARG, a nuclear receptor that binds prostaglandins and then affects gene expression downstream, is critical for pregnancy success, indicating the potential for intrinsic PGs to regulate mononuclear trophectoderm cells of the elongating conceptus. When meloxicam, a PTGS2 specific inhibitor, was infused into the uterine lumen of ewes from days 8-14 of pregnancy, PG production was inhibited in both the conceptus and the endometrium and conceptus elongation was severely stunted or completely inhibited [216].

Altogether, available studies supported the idea that PGs are critical for peri-implantation conceptus growth in sheep. In order to investigate the specific role of PTGS2-derived PGs in the conceptus, CRISPR-Cas9 genome editing was 
used to inactivate PTGS2 in the embryo and to determine effects on conceptus growth during early pregnancy.

\section{Materials and Methods}

\section{Targeting of PTGS2}

Two different sets of gRNAs were designed to target either exons 2 and 3 or exon 8 of the Ovis aries PTGS2 gene (Supplementary Table S1). Specificity of the gRNAs was checked by comparing the targeted sequence to similar ovine sequences containing a protospacer adjacent motif (PAM) domain in GenBank. Gene fragments gBlock $($ IDT, Coralville, IA) containing a T7 promoter, and gRNA containing the desired target sequence were in vitro transcribed using the MegaShortScript T7 Transcription kit (ThermoFisher, Waltham, MA) and purified using MEGAclear Transcription Clean-Up kit (ThermoFisher). gRNAs were quantified by Qubit RNA BR Assay (ThermoFisher) and stored at $-80^{\circ} \mathrm{C}$ for later use.

Sheep In Vitro Fertilization (IVF)

All reagents were purchased from ThermoFisher Scientific unless specified. Ovine ovaries were obtained from a local slaughterhouse and transported in warmed PBS $\left(\sim 38^{\circ} \mathrm{C}\right)$ to the laboratory within 2 hours of collection. In the lab, ovaries were briefly rinsed with warm PBS. All visible follicles within each ovary were sliced open and the ovary was washed in oocyte collection medium [OCM; medium 199 containing Hank's salts and L-glutamine, $350 \mathrm{mg} / \mathrm{L}$ sodium 
bicarbonate, $2.38 \mathrm{~g} / \mathrm{L}$ hepes, $1 \mathrm{x}$ glutamax, $100 \mathrm{IU} / \mathrm{ml}$ penicillin, $100 \mathrm{\mu g} / \mathrm{ml}$ streptomycin and supplemented with FBS (Peak Serum, Wellington, CO) and Heparin (8 USP Heparin and $2 \mathrm{ml} \mathrm{FBS/100ml} \mathrm{OCM)].} \mathrm{OCM} \mathrm{containing} \mathrm{COCs} \mathrm{was}$ filtered through a $100-\mu \mathrm{m}$ cell strainer, and the strainer was rinsed with warm OCM into a square grid plate for searching under a stereomicroscope (SMZ 800N, Nikon, Japan). Only oocytes with a homogeneous cytoplasm surrounded by at least two complete layers of cumulus cells were selected for in vitro maturation. Selected COCs were placed in groups of 10 in $500 \mu \mathrm{l}$ pre-equilibrated in vitro maturation medium [medium 199 with Earle's salts, L-glutamine and sodium bicarbonate containing $10 \%(\mathrm{v} / \mathrm{v})$ bovine steer serum, $100 \mu \mathrm{g} / \mathrm{ml}$ gentamicin, 100 nM sodium pyruvate, 1x glutamax, $50 \mu \mathrm{g} / \mathrm{ml} \mathrm{FSH}$ (Folltropin, Vetoquinol, Fort Worth, TX), $2 \mu \mathrm{g} / \mathrm{ml}$ estradiol and $0.01 \mathrm{mM}$ cysteamine] covered with mineral oil in a 2-ml tube and shipped overnight in an incubator at $38.5^{\circ} \mathrm{C}$ to the University of Missouri.

After 22-24 h of maturation, groups of approximately $20 \mathrm{COC}$ were washed 3 times in HEPES-TALP medium and placed in a $60 \mu \mathrm{l}$ drop of fertilization medium (SOF, $2 \%$ estrous sheep serum, $10 \mu \mathrm{g} / \mathrm{mL}$ heparin, and $10 \mu \mathrm{g} / \mathrm{mL}$ hypotaurine) [436, 437]. Sperm were purified from frozen-thawed straws as previously described and diluted in fertilization medium to achieve a final concentration of $1 \times 10^{6} / \mathrm{ml}$ in the fertilization drop [438]. Fertilization proceeded for 17 to $19 \mathrm{~h}$ at $38.5^{\circ} \mathrm{C}$ in a humidified atmosphere of $5 \%(\mathrm{v} / \mathrm{v}) \mathrm{CO}_{2}$. At the end of fertilization, putative zygotes (oocytes exposed to sperm) were denuded from the surrounding cumulus cells by vortexing for 5 min in $400 \mu$ l of HEPES-TALP. Zygotes were split 
in two groups: those to be injected with $40 \mathrm{ng} / \mu \mathrm{l}$ Cas 9 mRNA alone or $40 \mathrm{ng} / \mu \mathrm{l}$ Cas9 mRNA and 20ng/ $\mu$ l of each gRNA. Putative zygotes to be injected were placed in manipulation medium consisting of Tissue Culture Medium-199 with Hanks salts plus $0.595 \mathrm{mM} \mathrm{NaHCO}_{3}, 3.160 \mathrm{mM} \mathrm{HEPES}, 30.03 \mathrm{mM} \mathrm{NaCl}, 3 \mathrm{mg} / \mathrm{ml}$ bovine serum albumin, $0.05 \mathrm{mg} / \mathrm{ml}$ penicillin, and $0.06 \mathrm{mg} / \mathrm{ml}$ streptomycin). After injection, zygotes were cultured in four-well dishes in groups of up to 50 zygotes in $500 \mu \mathrm{l}$ of BBH7 (Cooley Biotech, FL, USA) covered with $300 \mu \mathrm{l}$ of mineral oil per well at $38.5^{\circ} \mathrm{C}$ in a humidified atmosphere of $5 \%(\mathrm{v} / \mathrm{v}) \mathrm{O}_{2}$ and $5 \%(\mathrm{v} / \mathrm{v}) \mathrm{CO}_{2}$. On day 7 morulas and blastocysts were collected and processed by adding $6 \mu \mathrm{l}$ Embryo Lysis Buffer (ELB) [40 mM Tris at pH 8.9, 0.9\% (v/v) Triton X-100, 0.9\% (v/v) Non-Idet P-40 (NP40), and 32 Units/ml of Proteinase K]. Embryos in ELB were put in the thermocycler for 30 mins at $60^{\circ} \mathrm{C}$, followed by $10 \mathrm{~m}$ at $85^{\circ} \mathrm{C}$. Lysed embryo solution was used as template for PCR analysis.

Animal husbandry and zygote editing

All experimental and surgical procedures were approved by the Institutional Animal Care and Use Committee of the University of Missouri-Columbia. Mature Rambouillet ewes (Ovis aries) were synchronized using a Controlled Internal Drug Releasing (CIDR) device (MWI Animal Health, Kansas City, KS) for 12 days (day $0=$ CIDR insertion). Ewes were superovulated from days $9-12$ by twice daily intramuscular injections of follicle stimulating hormone (Folltropin-V, Bioniche Animal Health, Belleville, ON, Canada) with decreasing amounts (34 mg, 26 mg, $18 \mathrm{mg}$, and $10 \mathrm{mg}$ ). On day 11, the CIDR was removed and ewes were given an injection of $15 \mathrm{mg}$ prostaglandin F2 $\alpha$ (Lutalyse, Zoetis, Parsippany-Troy Hills, New 
Jersey) and mated to fertile rams at onset of estrus. Embryos were collected at 36 h postestrus. Oviducts were flushed retrograde with $20 \mathrm{ml}$ of embryo flush media (Agtech, Manhattan, Kansas) from the uterotubal junction and recovered zygotes were immediately placed in $500 \mu \mathrm{l}$ of KSOMaa Evolve media (Zenith Biotech, Monkstown, Ireland). One cell presumptive zygotes were injected with $40 \mathrm{ng} / \mu \mathrm{l}$ Cas9 mRNA alone or $40 \mathrm{ng} / \mu \mathrm{l}$ Cas $9 \mathrm{mRNA}$ and $20 \mathrm{ng} / \mu \mathrm{l}$ of each gRNA in manipulation medium consisting of medium-199 with Hanks salts plus $0.595 \mathrm{mM}$ $\mathrm{NaHCO}_{3}, 3.160 \mathrm{mM} \mathrm{HEPES}, 30.03 \mathrm{mM} \mathrm{NaCl}$, and $50 \mu \mathrm{g} / \mathrm{ml}$ of gentamicin. After injection, zygotes were maintained in KSOMaa Evolve media with $4 \mathrm{mg} / \mathrm{ml}$ EFAFBSA (ThermoFisher), and $0.05 \mathrm{mg} / \mathrm{ml}$ gentamicin (Invitrogen) under mineral oil (Irvine Scientific, Santa Ana, California) for culture at 38.5 C in an atmosphere of $5 \% \mathrm{CO}_{2} / 5 \% \mathrm{O}_{2} / 90 \% \mathrm{~N}_{2}$ until transfer (day 8 ). Three to four blastocysts were surgically transferred into the uterus of synchronized day 8 post-estrus recipient ewes. On day fifteen after estrus, recipient ewes were euthanized, and tissues were immediately collected. The uterus was flushed with $20 \mathrm{ml}$ of sterile PBS as previously described [126], and the number of conceptuses recovered and their lengths were measured. The uterine lumen flush (ULF) was clarified by centrifugation and stored at $-80 \mathrm{C}$ until assay. Endometrium was physically dissected from the myometrium and flash frozen in liquid nitrogen for RNA extraction. Conceptuses were cultured for $12 \mathrm{~h}$ in DMEM/F12 (Thermofisher) alone with 1\% BSA fraction V (Sigma-Aldrich, St. Louis, Missouri). Media was collected and conceptuses were removed, snap frozen in liquid nitrogen, and stored at $-80^{\circ} \mathrm{C}$ 
for later isolation of DNA, RNA, and protein using an Allprep DNA/RNA/Protein Mini kit (Qiagen, Hilden, Germany).

Assessment of PTGS2 editing

Regions of PTGS2 surrounding the targeted areas were amplified from DNA (Supplemental Table S1) using TaKaRa Ex Taq proofreading DNA polymerase (Clontech). Amplified regions were visualized on an agarose gel and PCR amplicons were cloned into TOPO vectors (ThermoFisher) and transformed into DH5 $a$ competent cells. Clonal DNA was directly PCR amplified from eight colonies, cleaned using a QIAquick PCR Purification Kit (Qiagen) and sent for Sanger sequencing at the University of Missouri-Columbia DNA Core.

Western blot analysis of PTGS2

Western blot analysis was used to evaluate PTGS2 protein in recovered conceptuses. Protein was quantified using a Qubit protein assay (Thermofisher) and $15 \mu \mathrm{g}$ of protein per conceptus was loaded onto a mini-PROTEAN TGX precast gel (Bio-Rad, Hercules, California) and separated by SDS-PAGE at a constant voltage of $100 \mathrm{~V}$ for approximately 60 minutes in running buffer $(25 \mathrm{mM}$ Tris, $192 \mathrm{mM}$ glycine, $0.1 \%$ SDS). Next, protein was transferred onto a PVDF membrane in Towbin transfer buffer (25 mM Tris, $192 \mathrm{mM}$ glycine, 20\% methanol) at $100 \mathrm{~V}$ for 90 minutes. Membranes were blocked for $1 \mathrm{hr}$ in $5 \%$ milk in $0.1 \%$ TBST (1x Tris-Buffered saline containing 0.1\% Tween-20) and incubated overnight with a polyclonal PTGS2 antibody (160106; Cayman Chemicals) in a 1:200 dilution of blocking buffer. Membranes were rinsed with TBST three times and incubated 
with goat anti-rabbit horseradish peroxidase (HRP) conjugated secondary antibody at a 1:10,000 dilution for $1 \mathrm{~h}$ at room temperature. Membranes were rinsed in $0.1 \%$ TBST and then developed in SuperSignal West Pico Chemiluminescent Substrate (Thermo Scientific) for 5 minutes prior to visualization with a ChemiDoc MP System and Image Lab 4.1 Software (BioRad). Membranes were rinsed and then assayed for GAPDH using a monoclonal mouse GAPDH antibody (47724; Santa Cruz Technologies, Dallas, Texas) at 1:1000 dilution in $5 \%$ milk in $0.1 \%$ TBST. Membranes were incubated in a goat-anti mouse HRP conjugated secondary antibody at a 1:1,000 dilution for $1 \mathrm{~h}$ at room temperature. Membranes were developed as described for the primary antibody.

\section{Protein structure prediction}

Amino acid sequences for WT ovine PTGS2 was obtained from the NCBI database (NP_001009432.1). Edited PTGS2 sequences were entered into ExPASy Translate software to obtain the predicted amino acid sequence of edited conceptuses [439]. Next, both WT and edited PTGS2 amino acid sequences were entered into SWISS-MODEL software [440-444] to obtain predicted protein structures.

\section{Quantification of PGs}

Prostaglandins in the uterine flush and in individual conditioned media from conceptus culture was measured by enzyme-linked immunoassay from Cayman Chemical (51402) (Cayman Chemical, Ann Arbor, Michigan) as previously described [435]. This assay is highly reactive for most PGs. Samples (culture 
media) were diluted $1: 1000$ to fit within the assay range. Since prostaglandin production is contingent on conceptus size, total prostaglandin quantity was normalized for each conceptus by dividing the total amount of PGs produced by total conceptus protein.

\section{Quantification of IFNT}

The amount of IFNT in the uterine flush was determined by a western dot blot analysis using an antibody specific for IFNT raised in rabbits as previously described [435]. Protein content of ULF was quantified by Qubit protein assay. ULF protein $(20 \mu \mathrm{g})$ was loaded onto a nitrocellulose membrane in triplicate using a dot blot apparatus (Biorad) followed by rinsing with TBS. Membranes were blocked in $5 \%$ milk in $0.1 \%$ TBST and then incubated in IFNT antibody (1:1000) in blocking buffer overnight. Membranes were rinsed and then incubated in a goat-anti rabbit HRP conjugated secondary antibody at a 1:10,000 dilution. Samples were rinsed in $0.1 \%$ TBST and then developed in SuperSignal West Pico Chemiluminescent Substrate (Thermo Scientific) for 5 minutes prior to visualization with a ChemiDoc MP System and Image Lab 4.1 Software (BioRad). ImageJ software V 1.6 (National Institute of Health, Bethesda, MD) was used to quantify the relative amount of IFNT protein. The data are adjusted for the recovered volume of uterine flushing and expressed as total relative amount of IFNT in the uterine flush.

Total RNA isolation and analysis

Frozen endometrium was homogenized in TRIzol reagent (ThermoFisher) and total RNA was isolated using a chloroform-ethanol extraction protocol. 
Samples were treated with a DNAse I digestion and run through RNeasy MinElute cleanup columns (Qiagen). Quantity of total RNA was determined by Qubit RNA BR Assay and run on an agarose gel to assess RNA quality. Total RNA $(1 \mu \mathrm{g})$ from endometrium and conceptuses were reverse transcribed using the iScript RT supermix (Bio-Rad). Reverse transcription was performed following manufacturer's instructions. Control reactions were run in parallel without reverse transcriptase to test for genomic contamination. Real time PCR was done in triplicate on a CFX384 Touch Real Time System with SsoAdvanced Universal SyberGreen Green Supermix (Bio-Rad). PCR conditions were: activation, $95^{\circ} \mathrm{C}$ for 2 min; 40 cycles of $95^{\circ} \mathrm{C}$ for $5 \mathrm{sec} ; 60^{\circ} \mathrm{C}$ for 30 secs; and 72 for 30 sec. Previously published primers for PTGS2, prostaglandin-endoperoxide synthase 1 (PTGS1), IGFBP1, LGALS15, GRP, cathepsin-L (CTSL), solute carrier family 2 member 1 (SLC2A1), solute carrier family 2 member 5 (SLC2A5), and solute carrier family 5 member 1 (SLC5A1) were used [225], and primers for ovine PPARG were designed and synthesized by Integrated DNA Technologies (Supplemental Table S1). The $\Delta C T$ was the difference between the cycle threshold (CT) for the average of three housekeeping genes (Glyceraldehyde 3-phosphate dehydrogenase [GAPDH], succinate dehydrogenase complex flavoprotein subunit A [SDHA], and tyrosine 3-monooxygenase [YWHAZ]) and the gene of interest. For visualization fold change was calculated relative to the reference genes $\left(2^{-\Delta C T}\right)$. Gene expression was analyzed by ANOVA using the GLM procedure of SAS. 


\section{Results}

Testing PTGS2 guides in IVF produced embryos

The predicted Ovis Aries PTGS2 gene contains 10 exons that encodes two transcripts resulting in a protein of 604 or 607 amino acids. Two different sets of gRNAs were designed to either target exon 2 and 3 or exon 8 of the Ovis aries PTGS2 gene (Figure 1, Supplemental Table S1). The targeted region was chosen based on read mapping of PTGS2 mRNA from a day 14 ovine conceptus to Ovis aries reference genome (Brooks et al., 2016). Due to alternative exon usage between the transcripts, one set of guides were targeted to exon 2 and 3. Exon 8 was targeted because it contains the active site of cyclooxygenase activity [445]. To ensure guides cut efficiently, guides were first tested in IVF sheep embryos. The gRNA set targeting exon 8 did not cut efficiently, and thus, the gRNA set targeting exons 2 and 3 were used moving forward.

Ovis Aries PTGS2 gene

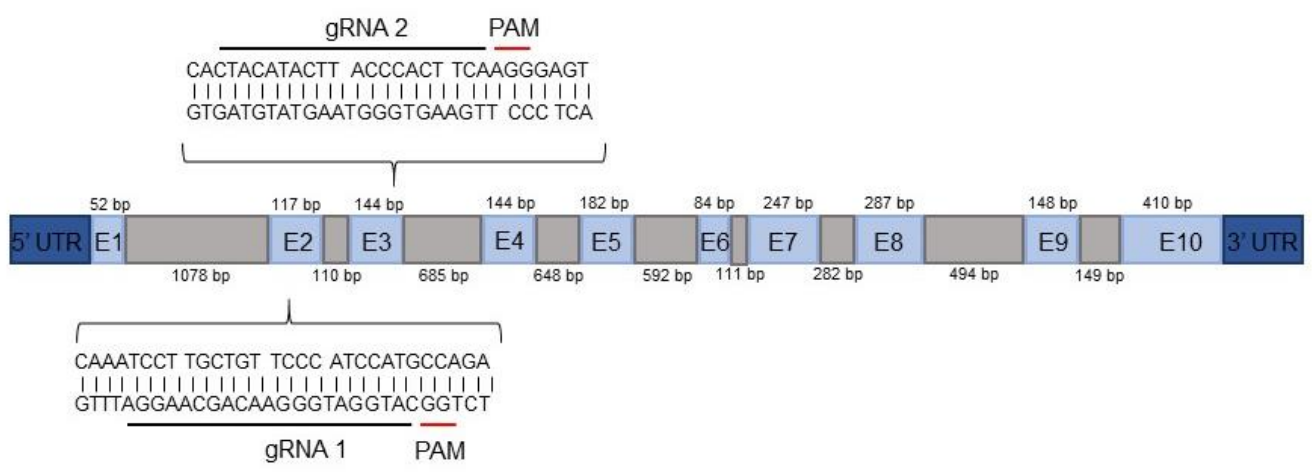

Figure 1. Structure and targeting of the Ovis Aries PTGS2 gene. The predicted Ovis Aries PTGS2 gene contains ten exons, two transcript variants, and encodes a protein made of 604 or 607 amino acids. Two different gRNAs were designed to target exon 2 and 3 of the Ovis Aries PTGS2 gene (depicted in red). 
Targeting of PTGS2 in in vivo produced embryos

In total, 12 PTGS2 targeted blastocysts were transferred and 10 conceptuses were recovered. PTGS2 was edited in all 10 recovered conceptuses (Figure 2a). Sanger sequencing indicated that all 10 of the conceptuses had biallelic edits as no wildtype sequence was identified in the conceptuses and 8 of the 10 sequences had multiple edits. The most common edits were a $1 \mathrm{nt}$ insertion near target sequences or a 287 nt deletion that spanned the splice site between exons 2 and 3 (Figure 2B). Five of the conceptuses had edits that would cause deletions in the PTGS2 gene without leading to frame shift mutations or premature stop codons (Figure 2C). These were included in downstream analyses.

Western blot analysis detected PTGS2 protein in 4 of the 6 Cas 9 control conceptuses and 1 of the 10 PTGS2 edited conceptuses (Figure 3A). In control conceptuses, the amount of PTGS2 protein was correlated to the length of the conceptus. One PTGS2 edited conceptus had an immunoreactive band of $\sim 60 \mathrm{kDa}$ in size. Sequencing and agarose visualization confirmed that this conceptus (\#8) had a biallelic 287 nt deletion that resulted in a 59 amino acid in-frame deletion. SWISS-MODEL software was used to predict protein structure for the PTGS2 edited conceptus \#8, which appeared altered and seemed to lack some of the structural integrity of the WT PTGS2 protein (Figure 3B). Due to the large deletion, this conceptus was included in later analyses as a PTGS2 edited conceptus. 


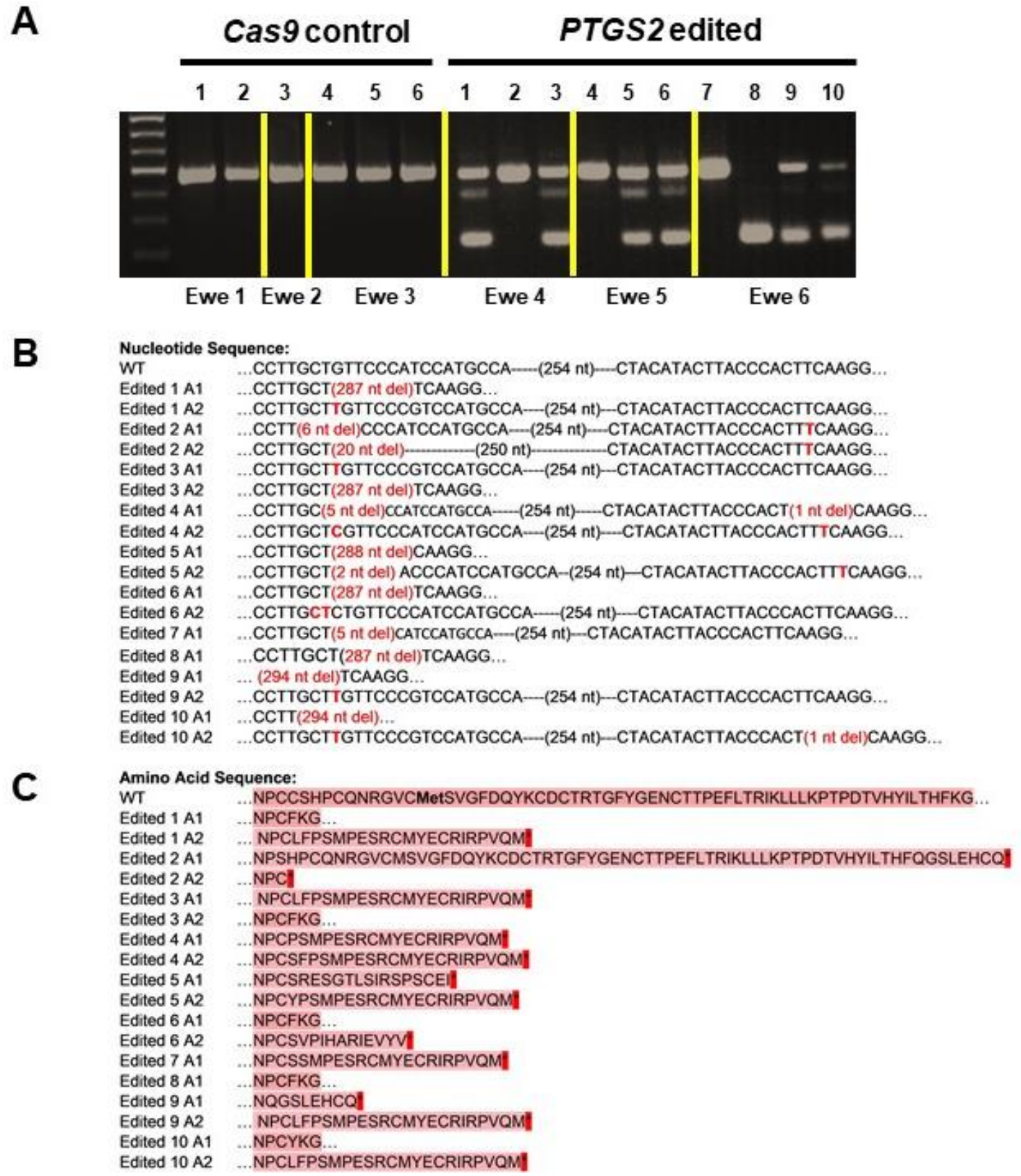

Figure 2. Design and effect of CRISPR gRNAs targeting the ovine PTGS2 for genomic editing. (A) DNA was isolated from recovered conceptuses and the targeted region in PTGS2 was amplified and run on an agarose gel to confirm editing. Yellow lines demarcate which ewe conceptuses were recovered from. (B) Amplified DNA was sent for sanger sequencing. All 10 of the recovered PTGS2 targeted conceptuses had bi-allelic alterations in the PTGS2 gene and 8 of the 10 had mosaic editing of the alleles (as indicated by A1 vs A2). (C) Amino acid sequence analysis indicated that PTGS2 targeted conceptuses had either a frameshift mutation and premature stop (indicated with a red asterisk) or a large amino acid deletion. 
A



B

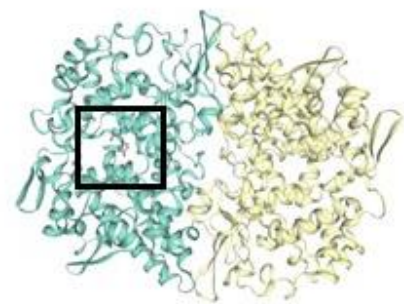

WT PTGS2 Structure

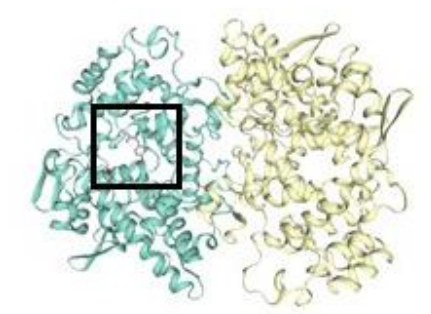

Conceptus \#8 PTGS2 Structure

C

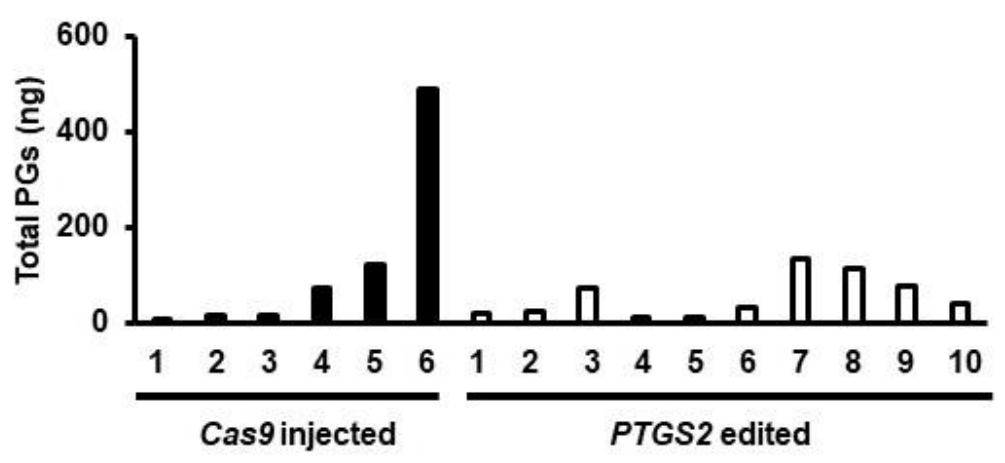

Figure 3. Effect of PTGS2 editing on PTGS2 protein and prostaglandin (PG) production. (A) PTGS2 is present in 4 of the 6 Cas 9 control conceptuses and is absent in 9 of the 10 PTGS2 edited conceptuses. (B) SWISS-MODEL software was used to predict the PTGS2 protein structure from a WT conceptus and biallelic edited conceptus \#8. PTGS2 is a homodimer and the two different colors demonstrate the different PTGS2 proteins. The box highlights the tyrosine residue responsible for cyclooxygenase activity, and its altered placement in the PTGS2 edited protein. (C) Conceptuses were individually cultured ex vivo and conditioned media was collected after 3 hours and used to determine PG production by individual conceptuses. PTGS2 targeted conceptuses produced fewer PG per $\mu \mathrm{g}$ of protein $(P=0.09)$. 
PTGS2 edited conceptuses produced fewer prostaglandins but elongated normally

Individual cultured PTGS2 edited conceptuses produced fewer prostaglandins per $\mu \mathrm{g}$ of protein $(P=0.09)$ (Figure $3 \mathrm{C})$. Overall, PTGS2 edited conceptuses were recovered from three ewes and Cas9 control conceptuses were recovered from three ewes. There was no difference between PG levels in the uterine lumen of the ewes who received Cas9 control (1410 $\mathrm{ng} \pm 489)$ as compared to PTGS2 edited conceptuses $(509.8 \mathrm{ng} \pm 145.4)(P=0.15)$ (Table 1). Cas9 control conceptuses and PTGS2 edited conceptuses were of similar length $(6.7 \mathrm{~cm} \pm 3.5$ vs. $6.5 \mathrm{~cm} \pm 1.1)(P=0.93)$. Total IFNT in the ULF was not different in ewes who received Cas9 control and PTGS2 edited conceptuses (Table 1).

Gene expression in endometrium of ewes who received Cas9 control versus PTGS2 edited conceptuses and in recovered conceptuses.

Endometrial expression of SLC2A1 was 3.5 -fold higher in ewes who received PTGS2 edited conceptuses $(P<0.01)$. There was no difference $(P>$ 0.10) in levels of PTGS2, PTGS1, IGFBP1, LGALS15, GRP, SLC2A5, SLC5A1, and SLC1A5 mRNA in the endometrium of ewes gestating Cas9 control and PTGS2 edited conceptuses (Figure 4A).

In the conceptuses, relative levels of PTGS2 and PPARG mRNA were not different. However, Cas9 control conceptuses had 2.5-fold higher $(P=0.050)$ expression of PTGS1 than PTGS2 edited conceptuses (Figure 4B). 
Table 1. Effect of PTGS2 gene editing on conceptus development and uterine PGs and IFNT'1.

\begin{tabular}{|c|c|c|c|c|}
\hline & \multicolumn{2}{|c|}{ Conceptus } & \multicolumn{2}{|c|}{ Uterine lumen } \\
\hline & Number & Morphology & $\begin{array}{c}\text { Total PGs (ng } \pm \\
\text { SEM) }\end{array}$ & $\begin{array}{c}\text { Total IFNT } \\
\left(R U \times 10^{3} \pm \text { SEM }\right)\end{array}$ \\
\hline Cas9 control & 6 & $\begin{array}{l}\text { Elongated and } \\
\text { filamentous }\end{array}$ & $1410.4 \pm 489.0$ & $11.0 \pm 6.5$ \\
\hline PTGS2 edited & 10 & $\begin{array}{l}\text { Elongated and } \\
\text { filamentous }\end{array}$ & $509.8 \pm 145.4$ & $18.6 \pm 4.1$ \\
\hline
\end{tabular}

A

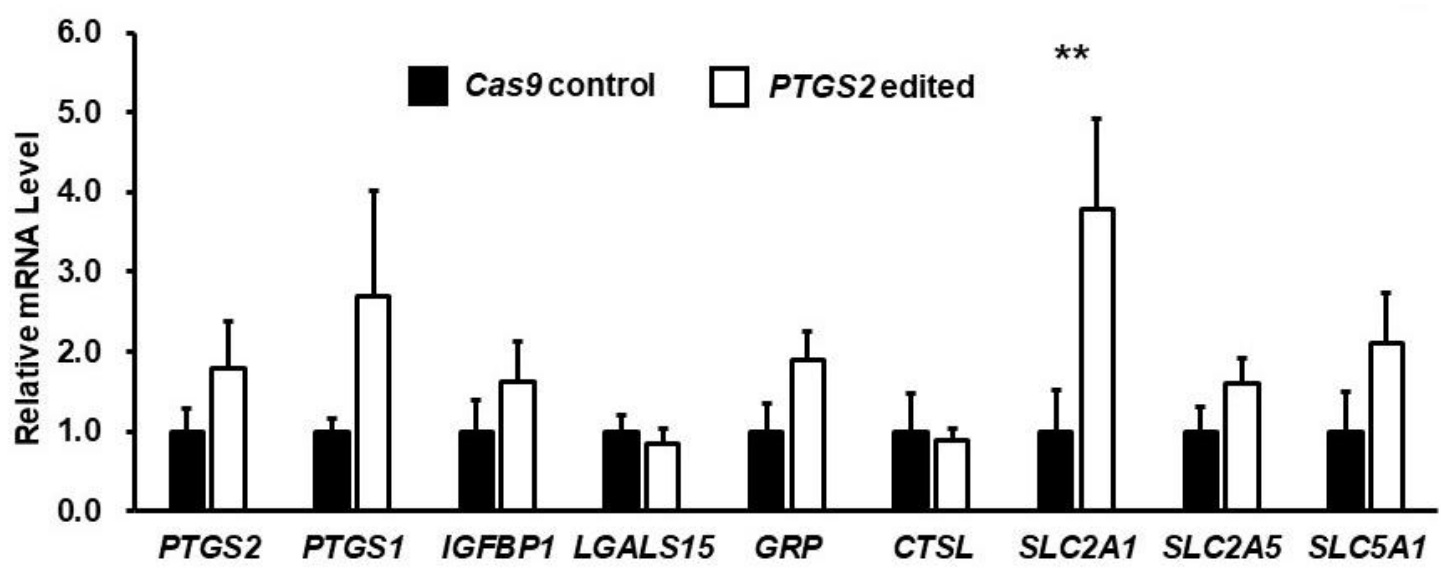

B



Figure 4. Effects of PTGS2 edited embryos on endometrial gene expression and gene expression in PTGS2 edited embryos. (A) Endometrial mRNA abundance was measured by real-time quantitative PCR and expressed as fold change of PTGS2 edited embryo transfer relative to Cas9 control embryo transferred ewes. (B) Conceptus mRNA abundance is expressed as fold change of PTGS2 edited relative to Cas9 control conceptuses. Significant differences are denoted by an asterisk $(P<0.05)$. Values are presented as least-squares means \pm SEM. 


\section{Discussion}

In ruminants, PTGS2 is expressed at the blastocyst stage (day 7) and can be used as a predictor of successful pregnancy in cattle [446, 447]. Sayre and Lewis [448] demonstrated that incubating ovine embryos with a nonselective PTGS inhibitor lowered blastocyst hatching rates. Here, the formation of blastocysts by PTGS2 edited zygotes and recovery of PTGS2 edited conceptuses suggests that PTGS2-derived PGs are not required for formation of a competent blastocyst or hatching from the zona pellucida.

The injection of two gRNAs efficiently targeted PTGS2 in all the recovered targeted conceptuses. Sequencing of PTGS2 edited embryos showed a variety of edits. The most common edits included a large deletion that spanned an intron, or a small 1 bp deletion that, based off the presumptive amino acid sequence from the start codon, would result in a frameshift mutation and premature stop codon. It is well established that CRISPR-Cas9 editing at the embryo stage can result in mosaic editing of the alleles $[449,450]$. Consistent with the literature, 8 of the 10 recovered PTGS2 edited conceptuses were mosaic with two or more different mutations, indicating biallelic editing. Nonetheless, all the edits would result in an inactive PTGS2 protein.

Quantitative PCR data and western blot analysis from recovered conceptuses indicated that PTGS2 deletion did not affect production of mRNA but did inhibit translation of PTGS2 protein. There was one PTGS2 edited conceptus that had successful translation, as indicated by an immunoreactive band to the PTGS2 antibody on western blot analysis at a lower molecular weight than Cas9 
control conceptuses. Interestingly, this conceptus had a large bi-allelic nucleotide deletion that spanned the splice site between exon 2 and 3 , demonstrating that transcription and translation of PTGS2 was still successful despite the interruption of the splice site by CRISPR-Cas9 gene editing. The tyrosine at the PTGS2 active site had altered placement in the PTGS2 edited conceptus and thus remained in the study [192].

It was previously reported that intrauterine infusion of a selective and specific PTGS2 inhibitor, meloxicam, considerably reduced total PGs in the uterine lumen, stunted conceptus elongation, and resulted in pregnancy loss by day 14 in sheep [216]. However, that study could not distinguish the specific role of PTGS2 in the conceptus as both the conceptus trophectoderm and endometrial epithelia express PTGS2 and produce large amounts of PGs [451]. The results presented here further delineate the role of PGs in the peri-implantation of pregnancy by demonstrating that PTGS2-derived PGs are not required for conceptus elongation. Instead, it appears that endometrial-produced PGs may play a critical role or that there may be some threshold of PGs in the uterine lumen for conceptus elongation that can be contributed by both the conceptus and endometrium. Since PGs are required for elongation, further work is necessary to determine the threshold of PGs required for successful pregnancy and if insufficient endometrial PG production may be an underlying cause of embryonic mortality in domestic species.

Despite inactivation of PTGS2, conceptuses made detectable amounts of PGs when cultured in vitro after collection. It is possible that conceptuses 
continued to synthesize PGs through a synthetic pathway involving PTGS1 or another enzyme. However, PTGS1 expression is low in ovine conceptuses during the peri-implantation period, as indicated by previous studies and gene expression analysis in this study [72]. There is significant evidence that PGs and enzymes in the PG biosynthetic pathway, such as PTGS1 and PTGS2, are present in extracellular vesicles (EVs) and may mediate the transcellular production of PGs [99, 452]. Indeed, EVs are present within the uterine lumen, are internalized by the developing conceptus, and contain the PTGS2 enzyme $[118,119]$. Thus, the uptake of EVs may supply the machinery to produce PGs in PTGS2 edited conceptuses, accounting for their PG production in vitro. Further, it is possible that PGs stored in the conceptuses are released during the brief in vitro culture period.

There were similar levels of PGs in the ULF between ewes that received Cas9 control and PTGS2 edited conceptuses, likely because the endometrium also produces and secretes large quantities of prostaglandins into the uterine lumen [433]. In this study, there were very few differences in endometrial gene expression between ewes gestating Cas9 control or PTGS2 edited conceptuses. The only gene that was different in the endometrium between ewes who received Cas9 control and PTGS2 edited conceptuses was SLC2A1 (GLUT-1), a major glucose transporter expressed in the uterine epithelia that increases around the time of conceptus elongation [139]. This difference in endometrial gene expression suggests that there was some difference in the uterine environment of ewes who received Cas 9 control versus PTGS2 edited conceptuses, leading to stimulation of this glucose transporter in the endometrium. Glucose in histotroph increases in 
the uterine lumen prior to conceptus elongation and stimulates proliferation of ovine trophoblast cells in vitro [179]. Considering that PGs are required for conceptus elongation and that PTGS2 edited conceptuses produce fewer PGs than their Cas9 control counterparts in vitro, perhaps SLC2A1 was upregulated in the endometrium to increase glucose within the uterine lumen to promote proliferation of the developing conceptus and offer a protective role against decreased PG production by the conceptus, promoting conceptus elongation.

Inactivation of PTGS2 did not affect IFNT production within the conceptus. It should be noted that IFNT is indicative of pregnancy success [216, 453], and the similar levels across the conceptuses suggest that that IFNT production was normal in all conceptuses and that early pregnancy events, through conceptus elongation, would proceed successfully. In mice, PTGS1 can compensate for PTGS2 to rescue fertility defects in the CD1 mice outbred strain [230]. Notably, this study suggests that PTGS1 does not compensate for PTGS2 expression in ovine conceptuses; in fact, PTGS1 was lower in PTGS2 edited conceptuses compared to Cas9 control conceptuses. PPARG is a nuclear receptor that is activated by PGs and is required for conceptus elongation and survival in sheep [435]. Although expression of PPARG was not affected by PTGS2 inactivation, it is plausible that PPARG signaling was altered since PTGS2 edited conceptuses produce fewer PGs and PGs activate PPARG signaling [204, 454]. Ovine conceptuses also express enzymes in the biosynthetic pathway of endocananbionids (ECBs) [72], and PTGS2 metabolizes ECBs, such as Narachidonylethanolamine (AEA) and 2-arachidonoylglycerol (2-AG), into PG- 
glycerol esters and PG-ethanolamides [455, 456]. Thus, PTGS2 inactivation may lead to higher levels of AEA and 2-AG in the conceptus, and like PGs, AEA and 2AG can activate PPARG signaling [457]. The potential of ECB accumulation due to PTGS2 inactivation in the ovine conceptus may have implications for later pregnancy success. In mice, ECB synthetic enzymes and receptors are tightly regulated in a spatiotemporal manner during embryo implantation and regulate the embryo implantation process [458, 459]. Thus, although PTGS2 edited conceptuses elongate normally, the effects of PTGS2 inactivation may manifest downstream during conceptus attachment and adhesion to the luminal epithelium.

In summary, this study provides the first evidence that intrinsic PTGS2derived PGs are not required for ovine conceptus elongation and IFNT production for maternal recognition of pregnancy. These results supports the idea that PGs from the endometrium are necessary and sufficient to support conceptus elongation during the peri-implantation period of pregnancy.

\section{Acknowledgements}

The authors appreciate assistance of all Spencer laboratory members and, specifically, Mr. David Todd for animal husbandry.

\section{Supplemental Information}

Supplemental Table 1. Guide RNA Sequences and primers for genotyping PTGS2 targeted conceptuses and qPCR analysis. 
Available online at:

https://doi.org/10.1002/mrd.23300 


\section{CHAPTER VI:}

\section{BIOLOGICAL ROLE OF APOA1 IN EARLY PREGNANCY IN SHEEP}

Eleanore V. O'Neil, M. Sofia Ortega, Joshua Benne, and Thomas E. Spencer

This work is under preparation for submission to Molecular Reproduction and

Development. 


\begin{abstract}
In sheep, conceptus elongation is regulated by substances present within the uterine luminal fluid. Select phospholipids and triglycerides are elevated in the uterine lumen of pregnant ewes, but it is unclear what signal mobilizes these lipids into the uterine lumen or how they are transported. The developing conceptus secretes substantial quantities of apolipoprotein A1 (APOA1), a lipid binding protein that promotes lipid efflux from tissues. Two studies were conducted to investigate biological roles of APOA1 in early pregnancy events. In study one, APOA1 was infused into the uterine lumen of cyclic sheep to determine if conceptus produced APOA1 mobilizes lipids into the uterine lumen. In study two, APOA1 was inactivated in embryos using CRISPR-Cas9 gene editing to investigate its role in pregnancy establishment. Infusion of APOA1 protein did not increase lipids within the uterine lumen or alter endometrial lipid droplets. Further, APOA1 production by the conceptus was not required for conceptus survival or development through day 27 . These results support the idea that conceptus derived APOA1 is not required for ovine conceptus survival or development through day 27.
\end{abstract}




\section{Introduction}

In domestic animals, pregnancy establishment encompasses conceptus elongation and maternal recognition of pregnancy signaling, implantation, and initial placentation. Within the uterine lumen, the ovine conceptus (embryo/fetus and its associated extraembryonic placental membranes) elongates between days 12 and 16 (day 0 = estrus) $[19,64]$. Implantation involves apposition, attachment, and adhesion of the conceptus trophectoderm to the luminal epithelial (LE) cells of the uterus [296, 302, 356, 357]. Trophoblast giant binucleate cells (BNC) begin to

differentiate on day 16 and are thought to form throughout pregnancy [34, 460]. Some BNC migrate and fuse with individual endometrial LE cells to form trinucleate feto-maternal hybrid cells, and BNC synthesize and secrete unique protein and steroid hormones such as pregnancy associated glycoproteins (or PAGs) and placental lactogen [460-462]. Conceptus elongation and subsequent development is largely dependent on regulation by the uterine environment, as embryos must be transferred to a recipient synchronized uterus to elongate $[65,348]$.

The molecular underpinnings of conceptus elongation and subsequent implantation and placentation are unclear. Conceptus elongation is likely regulated by nutrients and other macromolecules available to the conceptus in the uterine lumen $[72,119,138,359,361]$. Of the many factors that are in the uterine lumen, interest in lipids has been growing $[153,156,157,161]$. Lipids in the conceptus are likely very important, as they make up cell membranes and provide substrate for cell signaling and metabolism [160]. Select phospholipids are higher in the ULF of pregnant heifers compared to cyclic heifers $[153,156]$. However, the exact 
regulation of lipid homeostasis and transportation in the uterine lumen remains understudied.

Lipoproteins are one potential candidate for the transportation of lipids in the uterine lumen. The lipid composition of lipoproteins depends on the apolipoprotein component, which are proteins that stabilize lipids and determine whether the lipoprotein is predominantly made of triglycerides or cholesterol [233, 463]. In sheep, the conceptus upregulates several different apolipoprotein genes, including apolipoprotein $\mathrm{A} 1(A P O A 1)$ and apolipoprotein $\mathrm{E}(A P O E)$, during elongation and, most notably, APOA1 protein is higher in the uterine lumen of pregnant ewes than cyclic ewes on day $14[72,73]$. Interestingly, expression of $A P O A 1$ continues to increase in the conceptus after conceptus elongation and is expressed at a level similar to or above housekeeping genes (e.g. GAPDH) by day 20 , suggesting that it also may have important roles in later pregnancy events. In mice, endometrial secretion of APOA1 increases at the time of implantation, and in utero knockdown of APOA1 by siRNA resulted in reduced embryo implantations and increased pregnancy loss $[285,286]$. In serum, APOA1 is a core protein of high-density lipoproteins (HDL) and regulates reverse lipid transport, being secreted by the liver to mobilize phospholipids and cholesterol from peripheral tissues [464, 465]. Additionally, APOA1 and HDL associate with other proteins that can inhibit protease activity, regulate the complement system and decrease inflammation, and interact with endothelial cells [269, 463, 466, 467].

This evidence suggests that APOA 1 may play important roles in early pregnancy. Two studies were conducted here to determine if: 1) APOA1 secretion 
by the developing conceptus mobilizes endometrial lipids to the uterine lumen during conceptus elongation; and 2) to determine if APOA1 secretion by the conceptus has roles in embryo implantation or is critical for embryo survival through early implantation and placentation events.

\section{Materials and Methods}

\section{Study One}

All experimental and surgical procedures for the mature sheep (Ovis aries) were approved by the Institutional Animal Care and Use Committee of the University of Missouri. Adult cycling ewes were checked daily for estrus (day 0). Using a surgical approach described previously [69, 216, 364], ewes were subjected to a midventral laparotomy 10 days after estrus, and the lumen of each uterine horn received a vinyl catheter (0007760; Durect Corp, Cupertino, California) connected to an Alzet 2ML1 osmotic pump (Durect Corp) secured by suturing the oviduct and mesovarium to the outside of the uterus. Ewes received pumps containing the following: 1) vehicle; or 2) $0.2 \mathrm{mg}$ of APOA1 ( $\mathrm{n}=5 \mathrm{per}$ treatment). The Alzet $2 \mathrm{ML} 1$ osmotic pump has a reservoir volume of $2 \mathrm{~mL}$ and a pumping rate of $10 \mu \mathrm{L} / \mathrm{h}$ for 7 days. Ewes were euthanized on day 14 of the estrous cycle. The female reproductive tract was obtained through a mid-ventral incision. The oviduct and uterotubal junction was removed and a clamp was placed on the internal ostium of the cervix. A catheter attached to a syringe containing $10-\mathrm{ml}$ sterile MilliQ $\mathrm{H}_{2} \mathrm{O}$ was inserted about $1 \mathrm{~cm}$ into the uterine horn contralateral to the 
corpus luteum. Sterile $\mathrm{H}_{2} \mathrm{O}$ was instilled into the uterine lumen and gently massaged through the uterine body into and through the ipsilateral horn that was held over a sterile petri dish using a hemostat. The uterine lumen flush (ULF) was collected in the petri dish. The ULF was then transferred to a 15-ml tube, clarified by centrifugation $\left(3000 \times g\right.$ for $15 \mathrm{~min}$ at $\left.4^{\circ} \mathrm{C}\right)$, transferred into a clean $15-\mathrm{ml}$ tube, and volume recorded prior to storage at $-80^{\circ} \mathrm{C}$. Sections $(\sim 0.5 \mathrm{~cm})$ from the midportion of the uterine horn ipsilateral to the ovary were placed in optimum cutting temperature (OCT) compound (Sakura Finetek USA, Inc.), frozen over liquid nitrogen vapor, and stored at $-80^{\circ} \mathrm{C}$. The endometrium was physically dissected from the myometrium and flash frozen in liquid nitrogen for RNA extraction.

\section{Oil Red O (ORO) staining}

Uteri frozen in optimum cutting temperature compound were cryosectioned at $10 \mu \mathrm{m}$ on a model CM1950 cryostat (Leica Microsystems), mounted on slides, and stored at $-20^{\circ} \mathrm{C}$. Lipid droplets were stained as described [386]. Briefly, ORO stock solution was made by adding $2.5 \mathrm{~g}$ of ORO (Sigma-Aldrich, St. Louis MO) to 99\% Isopropyl alcohol (Sigma-Aldrich) and mixed for $2 \mathrm{~h}$ at room temperature. For staining, ORO stock was mixed in a 3:2 ratio with distilled water to make a fresh ORO working solution. After ten minutes at $4^{\circ} \mathrm{C}$, the ORO working solution was filtered $(40-\mu \mathrm{m})$. Frozen sections were equilibrated for $10 \mathrm{~min}$ at room temperature and then submerged in working ORO solution for 10 minutes. Sections were rinsed under running tap water for 30 minutes and mounted with Flouromount-G Mounting medium (ThermoFisher Scientific). Brightfield images were collected with a Leica DM5500 B upright microscope using Leica Application Suite X (LAS X) within $6 \mathrm{~h}$ 
of staining. To quantify staining, images were analyzed using ImageJ software. Briefly, the image was converted to an 8-bit grayscale format and the stained lipid droplets were segmented using thresholding. Threshold conditions were kept the same across all uterine sections. The area marked using the threshold conditions was calculated as a percentage of the total image. The average area covered was averaged between across the two groups.

Lipid extraction and MS analysis by multiple reaction monitoring (MRM)-profiling

For MS analysis, ULF was subjected to MS analysis by MRM-profiling at the Metabolite Profiling Facility of Purdue University. Lipids were extracted using the Bligh and Dyer method [316]. For that, $200 \mu \mathrm{L}$ suspension was transferred to a new microtube and mixed with $250 \mu \mathrm{L}$ of chloroform and $450 \mu \mathrm{L}$ of methanol and spiked with $100 \mathrm{ng}$ of phopspholipid internal standards (Avanti Equisplash Lipidomix standard). This solution was incubated at room temperature for $15 \mathrm{~min}$. After that, $250 \mu \mathrm{L}$ of chloroform and $250 \mu \mathrm{L}$ of water were added and the sample was centrifuged for $10 \mathrm{~min}$ at $16,000 \times \mathrm{g}$, forming a two-phase solution where the bottom phase contained the lipids (organic phase). The organic phase was transferred to a new tube and dried using a Speedvac centrifuge (Savant Speedvac, Thermo Scientific Inc., San Jose, CA, US), and samples were stored at $-80^{\circ} \mathrm{C}$ until MS analysis.

Lipid profiling was performed using the MRM-profiling method similar to that recently described [317]. Dried lipid extracts were diluted in $50 \mu \mathrm{L}$ of methanol/chloroform $3: 1 \quad(\mathrm{v} / \mathrm{v})$ and $250 \mu \mathrm{L}$ of injection solvent (acetonitrile/methanol/ammonium acetate $300 \mathrm{mM} 3: 6.65: 0.35(\mathrm{v} / \mathrm{v})$ ) to obtain a 
stock solution. Final dilution of the stock solution was performed to obtain $10 \mathrm{e} 6$ ion signal for the PC/SM lipids, which are the most abundant lipid classes. Mass spectrometry data was acquired by flow-injection (no chromatographic separation) from $4 \mu \mathrm{L}$ of diluted lipid extract delivered using a autosampler to the ESI source of an Agilent 6460 triple quadrupole mass spectrometer (Agilent Technologies, Santa Clara, CA, USA). A binary pump (Agilent 1200) was connected to the autosampler and operated at a flow rate of $20 \mu \mathrm{L} / \mathrm{min}$ and pressure of $30 \mathrm{bar}$. Capillary voltage on the instrument was $3.5-5 \mathrm{kV}$ and the gas flow $5.1 \mathrm{~L} / \mathrm{min}$ at $300^{\circ} \mathrm{C}$.

Study Two

Mature ewes (Ovis aries) were synchronized using a Controlled Internal Drug Releasing (CIDR) device (MWI Animal Health, Kansas City, KS) for 12 days (day $0=$ CIDR insertion). Ewes were superovulated from days $9-12$ by twice daily intramuscular injections of follicle stimulating hormone (Folltropin-V, Bioniche Animal Health, Belleville, ON, Canada) with decreasing amounts (34 mg, 26 mg, $18 \mathrm{mg}$, and $10 \mathrm{mg}$ ). On day 11 , the CIDR was removed and ewes were given an injection of $15 \mathrm{mg}$ prostaglandin F2 $\alpha$ (Lutalyse, Zoetis, Parsippany-Troy Hills, New Jersey) and mated to fertile rams at onset of estrus. Embryos were collected at 36 h postestrus. Oviducts were flushed retrograde with $20 \mathrm{ml}$ of embryo flush media (Agtech, Manhattan, Kansas) from the uterotubal junction and recovered zygotes were immediately placed in $500 \mu \mathrm{l}$ of KSOMaa Evolve media (Zenith Biotech, Monkstown, Ireland). One cell presumptive zygotes were injected with $50 \mathrm{ng} / \mu \mathrm{l}$ Cas9 protein alone or $50 \mathrm{ng} / \mu \mathrm{l}$ Cas9 protein and $25 \mathrm{ng} / \mu \mathrm{l}$ of each gRNA in 
manipulation medium consisting of medium-199 with Hanks salts plus $0.595 \mathrm{mM}$ $\mathrm{NaHCO}_{3}, 3.160 \mathrm{mM}$ HEPES, $30.03 \mathrm{mM} \mathrm{NaCl}$, and $50 \mu \mathrm{g} / \mathrm{ml}$ of gentamicin. After injection, zygotes were maintained in $\mathrm{BBH} 7$ media (Cooley Biotech, Gainesville FL) under mineral oil (Irvine Scientific, Santa Ana, California) for culture at $38.5^{\circ} \mathrm{C}$ in an atmosphere of $5 \% \mathrm{CO}_{2} / 5 \% \mathrm{O}_{2} / 90 \% \mathrm{~N}_{2}$ until transfer (day 8). Three to four blastocysts were surgically transferred into the uterus of synchronized day 8 postestrus recipient ewes. On day 21 and 27 post-estrus, recipient ewes were euthanized and placentas were carefully dissected out and photographed. Pieces of the placenta were dissected and frozen for RNA isolation and the fetus was fixed in formalin at room temperature for $24 \mathrm{~h}$, dehydrated through a series of ethanol and embedded in paraffin for histology.

Targeting of APOA1 for gene editing

Two gRNAs were designed to target intron 2 and exon 2 (Supplemental Table 1). Specificity of the gRNAs was checked by comparing the targeted sequence to similar ovine sequences containing a protospacer adjacent motif (PAM) domain in GenBank. Alt-R CRISPR-Cas9 crRNA (IDT) was ordered containing the targeted sequence and resuspended in resuspension buffer to 500 $\mathrm{ng} / \mu \mathrm{l}$. The crRNA was mixed at equal molar concentration with the tracrRNA to make a $250 \mathrm{ng} / \mu \mathrm{l}$ solution and then annealed in a thermocycler $\left(95^{\circ} \mathrm{C}\right.$ for $5 \mathrm{~min}$ and gradual ramp down to holding at $25^{\circ} \mathrm{C}$ ). The crRNA and tracrRNA hybrid was diluted to $25 \mathrm{ng} / \mu \mathrm{l}$ and with Cas9 protein $(50 \mathrm{ng} / \mu \mathrm{l})$ for 15 minutes at room temperature prior to injection.

Assessment of APOA1 editing 
Regions of APOA1 surrounding the targeted areas were amplified from isolated conceptus DNA (Supplemental Table 1) using TaKaRa Ex Taq proofreading DNApolymerase (Clontech). Amplified regions were visualized on an agarose gel and PCR amplicons were cloned into TOPO vectors(Thermo Fisher Scientific) an d transformed into DH5a competent cells. Clonal DNA was directly PCR amplified from eight colonies, cleaned using a QIAquick PCR Purification Kit (Qiagen), and sent for Sanger sequencing at the University of Missouri-Columbia DNA Core.

RNA extraction and analysis

Frozen endometrium was homogenized in TRIzol reagent (Thermo Fisher Scientific) and total RNA was isolated using a Direct-zol RNA MiniPrep Plus isolation kit (Zymo Research, R2070) on EconoSpin Columns (Epoch Life Science, Inc. Missouri City, TX). Samples were treated with an on-column DNAse I digestion and run through RNeasy MinElute cleanup columns (Qiagen). Quantity of total RNA was determined by Qubit RNA BR Assay and run on an agarose gel to assess RNA quality. Total RNA $(1 \mu \mathrm{g})$ from endometrium and conceptuses were reverse transcribed using the iScript RT supermix (Bio-Rad). Reverse transcription was performed following manufacturer's instructions. Control reactions were run in parallel without reverse transcriptase to test for genomic contamination. Real time PCR was done in triplicate on a CFX384 Touch Real Time System with SsoAdvanced Universal SyberGreen Green Supermix (Bio-Rad). PCR conditions were: activation, $95^{\circ} \mathrm{C}$ for $2 \mathrm{~min} ; 40$ cycles of $95^{\circ} \mathrm{C}$ for $5 \mathrm{sec} ; 60^{\circ} \mathrm{C}$ for 30 secs; and 72 for $30 \mathrm{sec}$. Previously published ovine and bovine primers for APOA1, vascular 
endothelial growth factor (VEGFA), tumor necrosis factor alpha (TNFA), glucose transporter type 4 (GLUT4), fatty acid binding protein 4 (FABP4), elongation of very long chain fatty acid (ELOVL), apolipoprotein $E$ (APOE), and peroxisome proliferator-activated receptor gamma (PPARG) were used [74, 359, 468, 469] (Supplemental Table S1). The $\Delta \mathrm{CT}$ was the difference between the cycle threshold (CT) for the average of two housekeeping genes (Glyceraldehyde 3-phosphate dehydrogenase [GAPDH] and tyrosine 3-monooxygenase [YWHAZ]) and the gene of interest. Melt curves were checked to ensure that a single product was amplified. For visualization fold change was calculated relative to the reference genes (2 $\Delta \mathrm{CT})$. Gene expression was analyzed by ANOVA using the GLM procedure of SAS.

\section{Histology}

Uteri were sectioned at $8 \mu \mathrm{m}$ and allowed to air dry for 10 minutes at $37^{\circ} \mathrm{C}$. Fetal sections were put through a series of dehydration and then stained with hematoxylin and eosin.

Statistical analyses

All quantitative data were subjected to least-squares ANOVA using the GLM procedures of the SAS (SAS Institute Inc., Cary, NC). In all analyses, error terms used in significance were identified according to the expectation of the mean squares for error. Significance $(P \leq 0.05)$ was determined by the probability differences of the least-squares means. 


\section{Results}

Study One

APOA1 protein was infused into the uterine lumen of cyclic ewes using osmotic pumps. Endometrial lipid droplets were present in the epithelium of ewes (Figure 1A) but infusion of APOA1 did not alter their abundance $(P=0.09)$ (Figure 1B). Genes involved in lipid metabolism (APOA1, FABP4, ELOVL), vasculogenesis (VEGFA), immune response (TNFA), and metabolite transportation (GLUT4) were evaluated by qPCR in the endometrium, but no gene expression differences were detected (Figure 1C).

A


B

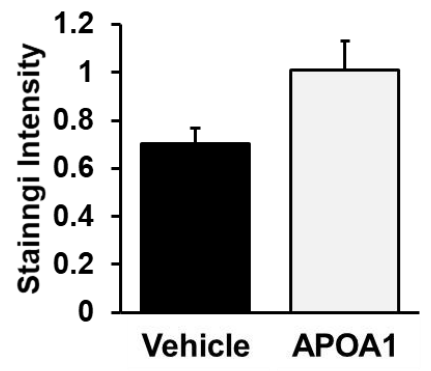

C

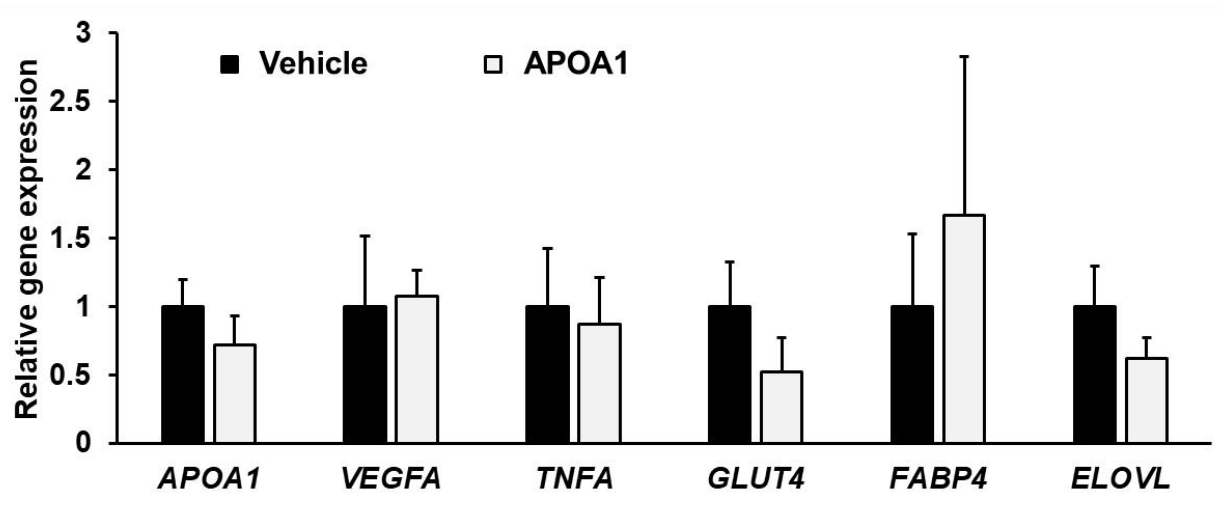

Figure 1. Effects of APOA1 infusion on epithelial lipid droplets and gene expression. (A) Uterine sections from vehicle and APOA1 infused ewes stained with Oil Red O stain. Scale bar: $100 \mu \mathrm{m}$. Infusion of APOA1 protein did not affect (B) endometrial lipid droplets or (C) endometrial gene expression. 
Next, lipids in the uterine lumen were compared. No differences were detected across the various lipid groups examined including phospholipids (e.g. phosphatidylcholine, phosphatidylethanolamine, phosphatidylinositol, phosphatidylglycerol), triglycerides, free fatty acids, cholesterol, and ceramides (Supplementary Table 2). Ewes who received either vehicle or APOA1 protein infusions clustered together by the top 25 most different lipids, which were predominantly triglycerides and acylcarnitines (Figure 2). Thus, APOA1 infusion induces subtle changes in the lipidome of the uterine lumen.

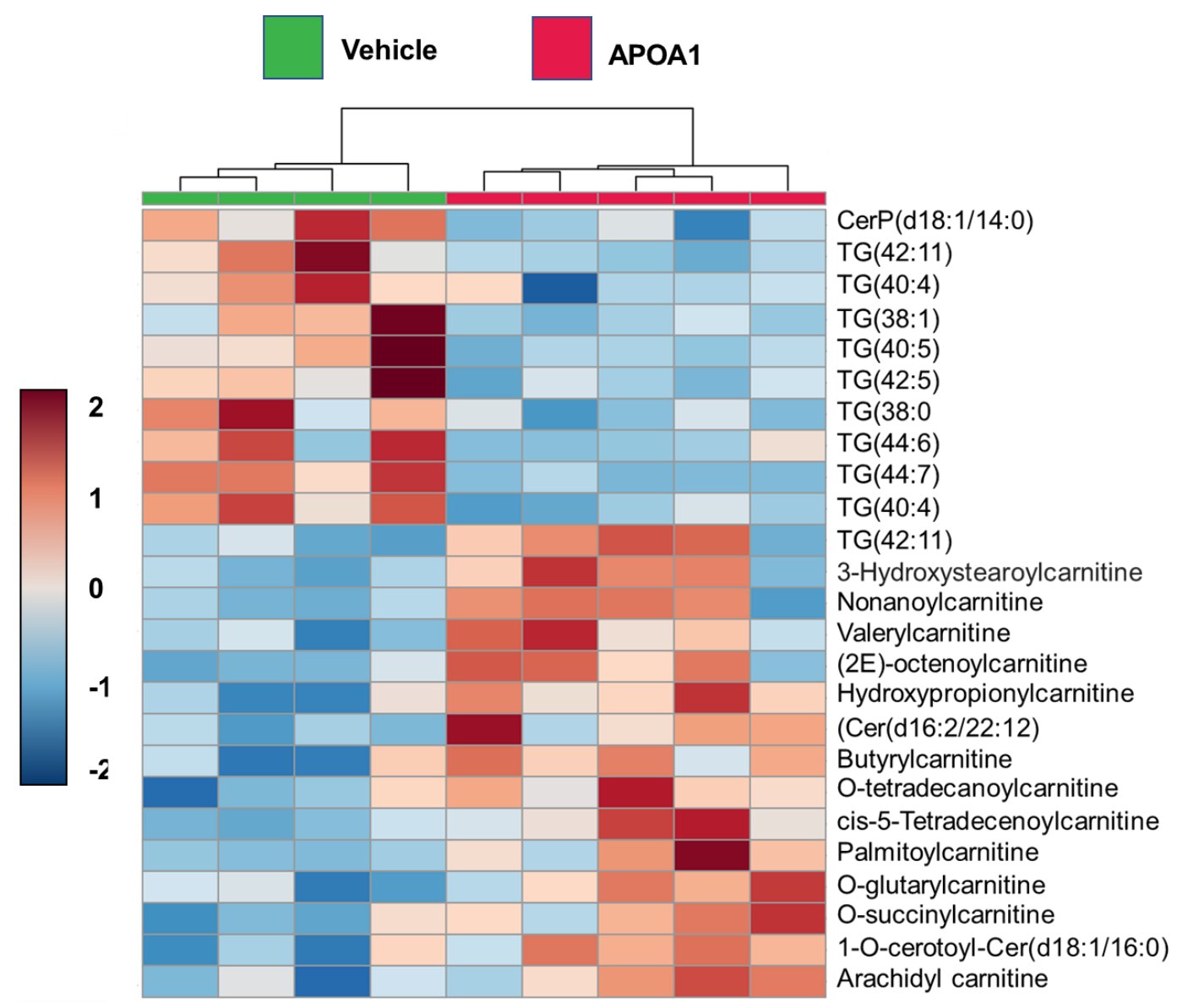

Figure 2. Heatmap analysis of TG in the ULF of vehicle infused (green) and APOA1 infused (red) sheep. 


\section{Study Two}

The Ovis Aries APOA1 gene contains 4 exons that encode a predicted 260 amino acid protein. One set of guide RNAs (gRNAs) was designed to target the first intron and the second exon (Figure 3A). This region was chosen in order to interrupt the splice site and splicing of the $A P O A 1$ gene. First, conceptuses were collected on day 21 to determine if they elongated normally and to see if the initial steps of implantation were successful. Conceptuses appeared normal and healthy, indicating that conceptus-derived APOA1 is not required for conceptus elongation (data not shown).

On day 27, four Cas9-injected fetuses were recovered of seven transferred (57\%) and nine APOA1-targeted fetuses were recovered of sixteen transferred (56\%). Sanger sequencing found that all 9 fetuses had edits. Wildtype sequences were detected in two of the APOA1-targeted fetuses (40B and $83 \mathrm{~A}$ ), although wildtype sequence was only one of the eight topocloned sequences of $83 \mathrm{~A}$ and thus could be attributed to maternal contamination. In total, eight of the APOA1targeted fetuses were expected to be biallelic knockouts (Figure 3B).

Quantitative PCR analysis showed that APOA1 was inhibited at the level of transcription as its expression was significantly reduced $(P<0.01)$ compared to Cas9-injected controls (Figure 4). Other genes measured included ones involved in vasculogenesis, immune response, and lipid metabolism. While most genes were not affected by APOA1 targeting, TNFA expression was reduced in APOA1edited conceptuses $(P<0.01)$, indicating that APOA1 affects inflammatory pathways in the placenta. 


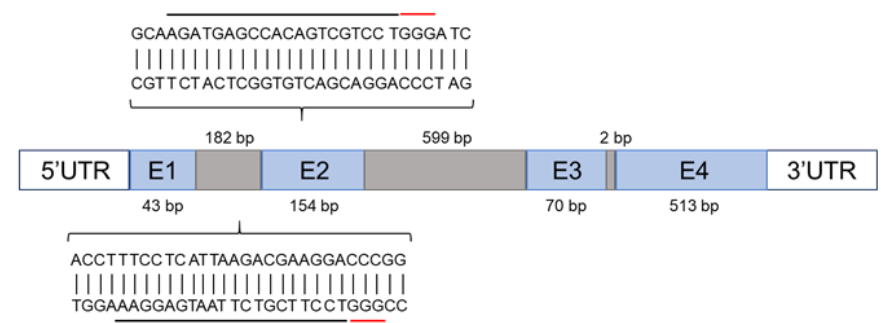

B

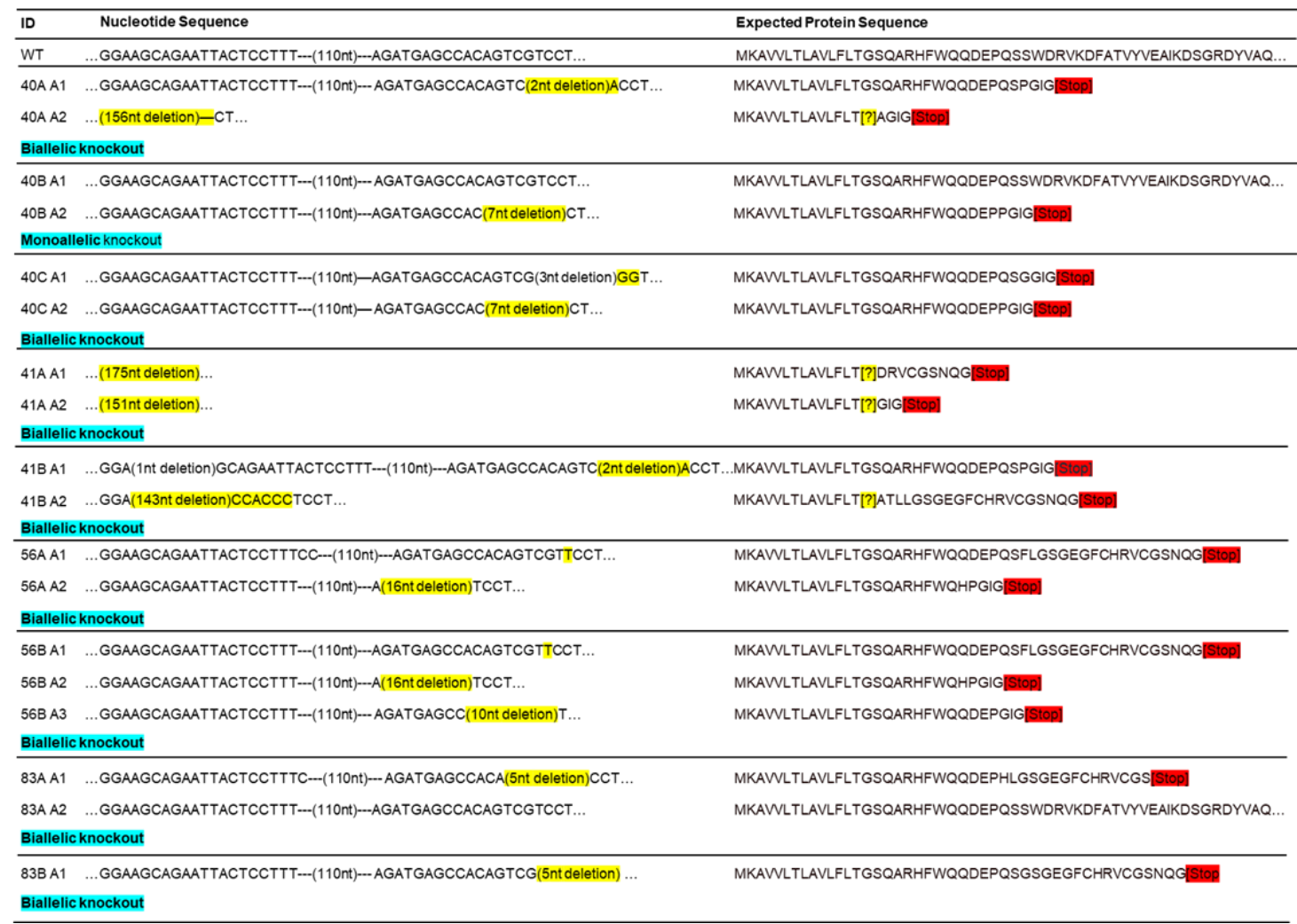

Figure 3. APOA1 gene structure and CRISPR-Cas9 gene editing effects. (A) Structure and targeting of the Ovis aries APOA1 gene. The APOA1 gene contains 4 exons (light blue) and three introns (gray) and encodes a 260 amino acid protein. Two gRNAs, as marked by a black line, were designed to target intron 2 and exon 2. The PAM sequence is denoted in red. (B) Effect of CRISPR gRNAs targeting the APOA1 ovine gene. The ID represents the ewe ID (number), conceptus ID (letter), and the unique allele found (A1, A2, or A3). Indels due to genomic editing are highlighted in yellow, and the expected protein sequence is described. Based off sanger sequencing results and resultant protein sequence, the expected effect (monoallelic or biallelic knockout) is indicated in blue. APOA1, apolipoprotein A-1; gRNA, guide RNA; PAM, protospacer adjacent motif. 


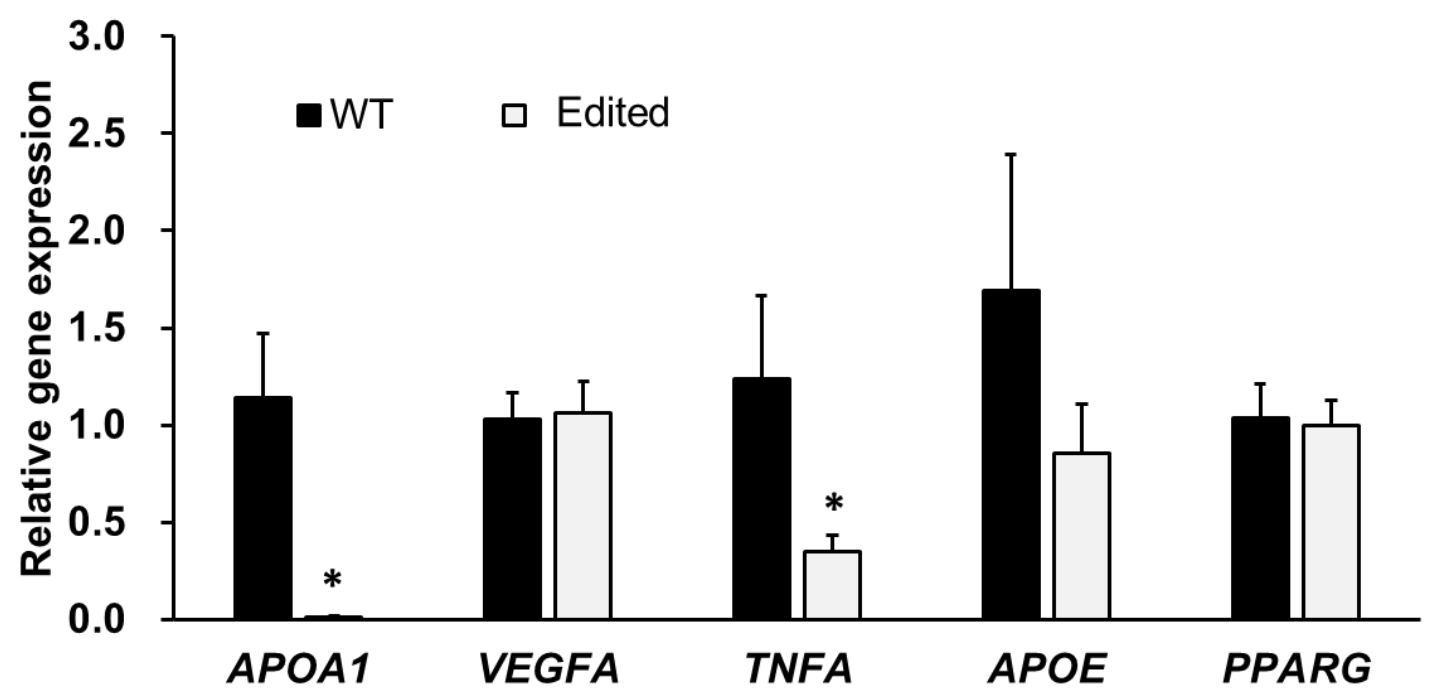

Figure 4. Effects of $A P O A 1$ editing on placental gene expression in APOA1 targeted conceptuses. Significant differences are denoted by an asterisk $(p<$ 0.05).

In total, seven of the nine APOA1-targeted fetuses were visually similar to Cas9-injected controls at time of collection (Figure 5A). However, two of the eight biallelic edited fetuses (56A and 56B) recovered from the same ewe were being resorbed on day 27 (Figure 5B). While all the wildtypes appeared normal at time of collection, fetal histology showed that one of these was not histologically normal. Based on somite number and size, APOA1-targeted fetuses were developmentally similar to Cas9-injected controls on day 27 (Figure 5C). 

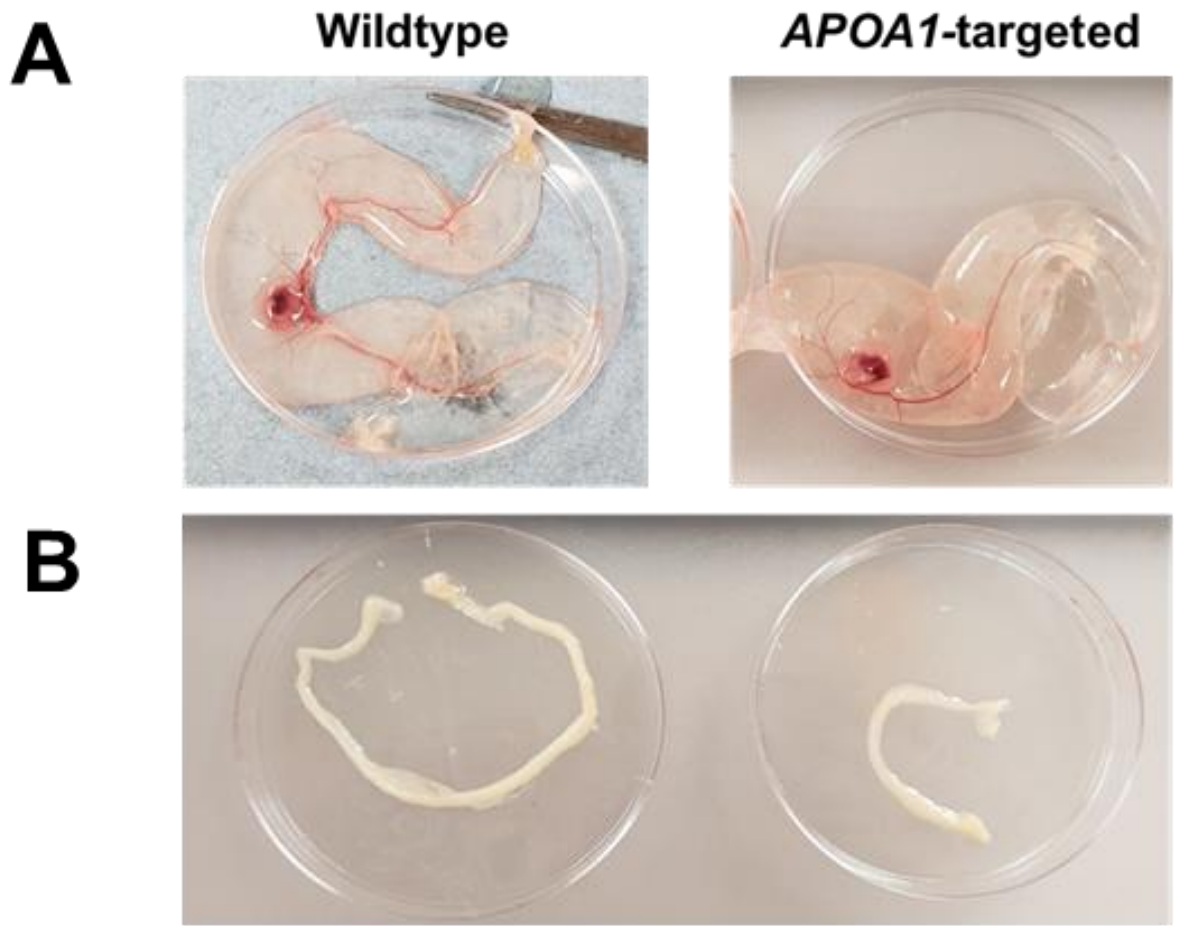

C

\section{Wildtype}

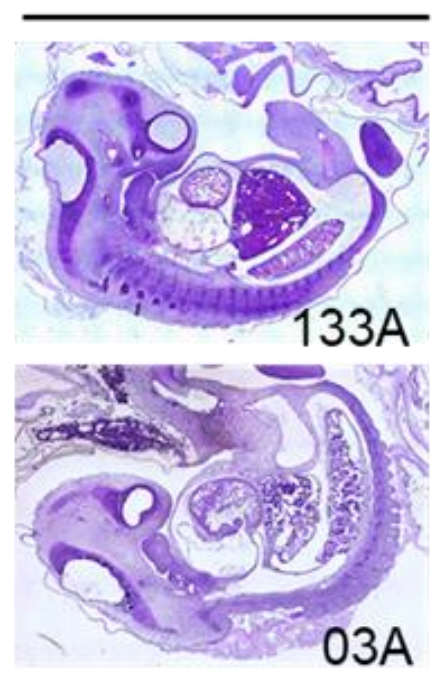

APOA1-Targeted
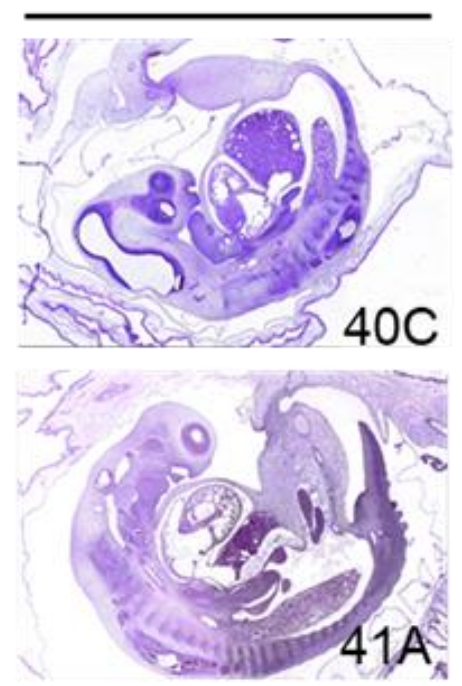

Figure 5. Effects of APOA1 editing on fetal and placental development. (A) Representative images of feto-placental units at time of collection. Cas9-injected controls and APOA1-targeted conceptuses appeared visually similar. (B) Two of nine APOA1-trageted conceptuses, transferred into the same ewe, were being resorbed on day 27. (C) Representative images of wildtype and APOA1-targeted conceptuses. Histological examination of $A P O A 1$-targeted conceptuses indicated that they were developmentally similar to Cas9-injected controls and were similarly sized. The ID indicated represents the ewe ID (number) and conceptus ID (letter). APOA1, apolipoprotein A-1. 


\section{Discussion}

Current evidence supports the hypothesis that lipids play important biological roles in conceptus elongation and development. Growing evidence supports the hypothesis that the endometrium transports lipid molecules to the conceptus [156, 160,161, 402]. Due to the hydrophobic nature of lipids, lipids must be packaged into lipoproteins or other lipid bound macromolecules to be transported in aqueous environments [233, 470]. To investigate whether conceptus production of APOA1 might be one molecule driving endometrial mobilization of phospholipids or other lipids into the uterine lumen, human APOA1 was infused into the uterine lumen of ewes. Human APOA1 protein has high similarity $(85 \%)$ to the sheep APOA1 protein and thus was expected to act similarly. Vehicle and APOA1 infused ewes did cluster together by heat map analysis of the top 25 most significant lipids, indicating there are subtle shifts in the lipidome of the uterine lumen. However, infusion of APOA1 did not significantly alter individual lipids or total phospholipids in the uterine lumen and did not affect endometrial lipid droplets. Thus, APOA1 protein alone does not appear to be a driving molecule for mobilizing lipids into the uterine lumen. Additionally, APOA1 did not alter endometrial genes involved in vasculogenic, inflammation, or metabolite transportation.

APOA1 is predominantly secreted into plasma as a lipid-poor APOA1 protein and lipids (predominantly phospholipids and cholesterol) are only added after interaction with ATP-binding cassette transporter sub-family A member 1 (ABCA1) or sub-family G member 1 (ABCG1) in tissues [268, 463-465, 471, 472]. 
Transcriptomic analysis of the bovine endometrium found that both $A B C A 1$ and $A B C G 1$ are expressed in the bovine endometrium [140]. However, conceptusproduced APOA1 would most likely interact with the surface of the LE and GE and analysis of the uterine epithelial transcriptome in sheep found $A B C A 1$ expression is exclusive to the GE and that $A B C G 1$ is not expressed in either LE or GE [72]. Thus, it is unclear if the required interacting molecules would be present or abundant enough on the surface uterine epithelium to mobilize endometrial lipids to conceptus produced APOA1.

Although APOA1 did not appear to mobilize endometrial lipids into the uterine lumen during conceptus elongation, it is still possible other apolipoproteins play this role. The ovine conceptus expresses $A P O B, A P O E, A P O A 2, A P O M$, among others, and APOE protein and APOA2 protein were also identified in the uterine lumen although they were not different between cyclic and pregnant ewes $[72,73]$. The predominant lipid type in the conceptus is triglycerides which are typically transported by lipoproteins other than HDL, such as the APOE-bound very low density lipoprotein [Chapter One, [233, 463]. Additionally, the endometrium synthesizes various apolipoproteins as well [140, 286]. Future work should characterize the various lipoproteins present within the uterine lumen and investigate their role in lipid transportation within the uterine lumen. Alternatively, lipoproteins may not have a role in mobilizing endometrial lipids during conceptus elongation; instead, epithelial lipid droplets may be secreted directly into the uterine lumen similar to their secretion by mammary epithelial cells [400, 401]. 
Finally, these results may indicate that APOA1 may not be produced to interact with the endometrium. The conceptus expresses both $A B C A 1$ and $A B C G 1$, suggesting the ability of the conceptus itself to interact with the APOA1 it produces [72]. In humans, APOA1 production is a marker of embryo quality in embryos and embryo uptake of APOA1 was associated with increased pregnancy success in IVF, suggesting that it has functions intrinsic to the embryo itself [289, 473]. It is possible that APOA1 regulates lipid homeostasis in the developing conceptus. Furthermore, APOA1 expression by the endometrium is increased during the period of embryo implantation in mice and infusion of siRNA targeting APOA1 decreases rates of embryo implantation [285, 286]. Thus, APOA1 may play important roles in conceptus implantation, early placentation, or survival.

To determine if APOA1 plays important roles in conceptus and fetal development, APOA1 was inactivated using the CRISPR-Cas9 system. Guides showed high editing efficiency and successfully generated APOA1-edited conceptuses. Pregnancies were terminated on day 27 to determine if fetuses failed to complete implantation or to initiate placentation. While two of the eight suspected biallelic-APOA1 knockout conceptuses were being resorbed, most had successfully implanted and initiated placenta development like the Cas9-injected controls. Additionally, the two degrading APOA1-edited conceptuses were recovered from the same uterus so the uterine environment itself could have been the underlying cause of pregnancy loss. Further examination and histology of the fetuses determined that $A P O A 1$-targeted fetuses were developmentally similar 
(around the 20-25 somite stage) to Cas9-injected controls. Thus, APOA1 is not required for conceptus implantation or early placentation through day 27.

Quantitative PCR (qPCR) was used to analyze effects of APOA1 inactivation on vasculogenesis (VEGFA), inflammation (TNFA), and lipid metabolism (APOE, PPARG) in the placenta on day 27. These genes were of interest because APOA1 promotes angiogenesis and reduces inflammation due to direct interaction with immune cells [474-476]. Interestingly, TNFA expression was lower in the placenta of $A P O A 1$ edited conceptuses, suggesting that APOA1 may have pro-inflammatory roles in placental development. It is unclear if this is due to direct regulation by APOA1 or if it is a downstream effect of APOA1 roles. As TNFA is predominantly produced by macrophages and APOA 1 is known to interact with macrophages, future studies should look into interactions of conceptus derived APOA1 and placental macrophages [477].

Finally, APOA1 protein is present in the uterine lumen of cyclic sheep, indicating that APOA1 does not solely come from the fetus but is also produced by maternal tissue $[73,478]$. Thus, it is possible that APOA1 plays critical roles for pregnancy survival to day 27 but that maternal APOA1 protein can compensate for insufficient production by the conceptus. Future work should clarify the role of endometrial APOA1 production and determine if complete knockdown of APOA1 within the uterine environment results in pregnancy loss.

Taken together, APOA1 is a heavily secreted protein by the developing ovine conceptus that has been demonstrated to have roles in embryo survival or competence in other species. The present studies indicate that conceptus derived 
APOA1 does has minimal roles in altering the lipidome of the uterine lumen and that conceptus APOA1 is not required for implantation or early placentation. Future work should focus on investigating the potential interaction of fetal APOA1 protein and placental macrophages as TNFA was dysregulated in APOA1 edited conceptuses. Additionally, more work should be done to understand the role of endometrial produced APOA1 and the role of lipoproteins in early pregnancy events. Collectively, these studies support the working hypothesis that the fetus produces factors that interact with and regulate its own development.

\section{Acknowledgements}

We thank David Todd for his expert assistance with sheep husbandry and experiments.

\section{Supplemental Information}

Supplemental Table 1. Lipidome of ULF of ewes who received vehicle or APOA1 protein infusions.

\section{Available upon request or after publication at https://onlinelibrary.wiley.com/journal/10982795}




\section{SUMMARY AND CONCLUSIONS}

In sheep, conceptus elongation is regulated by factors in the ULF that originate from the endometrial epithelium as well as the conceptus itself $[64,67$, 68]. These factors alter epithelial gene expression and regulate developmental processes in the conceptus important for elongation, including TE metabolism, proliferation, migration, attachment, and adhesion [296, 356]. Since embryos fail to develop past the blastocyst stage in vitro, it is essential to understand the components and the functional roles of ULF histotroph to understand what creates a successful pregnancy and why pregnancy loss at this stage may occur. Most studies have characterized the proteome and a few select metabolites within the ULF; however, the composition of the ULF is much more complex. An understanding of the presence and roles of ULF components beyond proteins during the peri-implantation pregnancy is lacking. Collectively, the studies in this dissertation support the central hypothesis that lipids and lipid associated macromolecules are key elements of the ULF that mediate endometrial-embryonic crosstalk and regulate conceptus development.

Extracellular vesicles are present within the uterine lumen of sheep and increase between days 10 and 12 of the estrous cycle $[119,126]$. Previous studies have characterized the RNA and protein cargo of uterine EV, demonstrated that both the conceptus and endometrium can internalize EV, and observed biological

effects of EV on the conceptus trophectoderm in sheep [118, 120]. Although EV are hypothesized to be mediators of endometrial-embryonic interactions during conceptus elongation, their regulation, makeup, and biological effects are not fully 
understood. In Chapter Two, EV are less abundant in the ULF of pregnant ewes versus cyclic ewes on days 12 and 14. It was critical to determine if EV are less abundant in the pregnant ULF due to uptake by the conceptus and endometrium or due to an inhibition in their production during early pregnancy. The protein ISG15 is a potent negative regulator of EV release and is stimulated in the endometrial stroma by IFNT in pregnant ewes $[58,81]$. To test if IFNT production by the conceptus prevents the release of EVs by the endometrium, IFNT was infused into the uterine lumen of cyclic sheep. However, IFNT infusion did not affect EV secretion into the ULF, probably because ISG15 is not expressed in the endometrial epithelium, which is the primary source of EV [58]. Thus, EVs are most likely depleted in the ULF of pregnant ewes due to uptake by the conceptus and endometrium. The RNA, protein, and lipid cargo of EV likely plays essential roles in recipient cell biology and provide significant substrate for the trophectoderm. The RNA and miRNA makeup of uterine EVs has been described in sheep and encodes enzymes, growth factors, and adhesion factors, as well as ribosomal RNA $[118,119,126]$. In this second Chapter, proteins were detected in uterine EVs that likely could alter the conceptus TE and endometrium. For example, EVs were replete with ribosomal proteins that may enhance the ability of either the conceptus or endometrium to translate proteins. Further, the lipid cargo of EVs isolated from ULF was described for the first time in any species. Uterine EVs contain phospholipids, ceramides, acylcarnitines, and PGs which may affect recipient cell biology. To understand the cumulative effects and importance of EV and their cargo, ovine trophectoderm cells and the endometrium were treated with EVs 
isolated from cyclic and pregnant ewes. In Chapter Two, treatment of ovine TE cells with EVs increased IFNT production and inhibited cellular proliferation. In Chapter Three, EVs regulate gene expression and PG production by the endometrium and mediate IFNT signaling. These results are consistent with other reports that have found biological effects of EV exposure on both endometrial epithelial cells and embryonic cells in vitro [120, 123, 134]. Taken together, EVs play important biological roles in early pregnancy as regulators of developmental processes and mediators of endometrial-embryonic crosstalk.

As EV refer to a heterogeneous mixture of EVs, future work should focus on understanding the subpopulations of EVs present within the uterine lumen and understanding if they have differential roles. These studies could clarify if there are specific subpopulations that are essential for pregnancy or if the various populations have overlapping functions. Although EVs have biological effects on the TE and endometrium, it is unclear what mediates these effects. The biological effects elicited specifically by the lipid, protein, or nucleic acid cargo warrants further examination. The development of liposomes with more biologically relevant lipid makeups could be used to parse out the biological effects of EV lipids in early pregnancy events compared to some of the nucleic acid or protein cargo [479]. Finally, the overall importance of EVs still needs to be assessed. Ideally an experiment could be conducted where EV release is prevented to see if embryo development if negatively affected. However, as EVs are made by many different synthetic pathways, there are not currently any drugs or methods to completely 
ablate EV release. As more studies characterize EV populations and their synthetic pathways, these experiments may eventually become achievable.

At the onset of conceptus elongation, glucose and amino acids are transported into the ovine uterine lumen to support conceptus metabolism and regulate conceptus development through MTOR activation [138, 139, 145]. In Chapter Four, the comprehensive lipidome and metabolome of the ULF, endometrium, and conceptus was investigated for the first time and compared between cyclic and pregnant ewes. These results expand our understanding of the peri-implantation uterine environment and highlight key changes in the pregnant uterus that may play important roles during pregnancy. While the endometrial lipidome and metabolome is relatively similar between cyclic and pregnant ewes, several classes of lipids and various metabolites are elevated in the ULF of pregnant ewes. Principal component analysis of the lipidome suggests that the endometrium is the primary driver of lipids in the ULF, as the lipid makeup of the endometrium and ULF is very similar while the conceptus lipidome is quite distinct. In contrast, the metabolome of the ULF is equally distinct from the endometrium and the conceptus, possibly indicative of embryonic and endometrial contribution or turnover of the ULF. The presence of phospholipids and TAGs in the ULF, which is an aqueous substance, indicates that EVs, lipoproteins, and/or other lipidassociated macromolecules are present within the uterine lumen and are available for use by the conceptus. These changes in the ULF makeup of pregnant ewes suggest that the ULF may provide a significant source of lipids and metabolites available to the conceptus. Further, the lipid and metabolite makeup of the ovine 
conceptus was reported for the first time and has implications for substrate uptake and use during conceptus elongation. Notably, TAGs may be mobilized from the endometrium into the ULF and are enriched within the conceptus. Overall, these findings highlight the complexity of the uterine environment and support the hypothesis that the endometrium mobilizes a wide variety of substances, including lipids and metabolites, into the ULF for the conceptus. These lipids and metabolites likely play critical roles in creating an environment that is conducive to embryo development and pregnancy success.

Mining of the lipidomic and metabolomic data could identify specific lipids and metabolites to further investigate as regulators of conceptus development in future studies. As the lipid makeup of the conceptus suggests that the conceptus does not passively internalize lipids from the ULF, future studies could use fluorescently-marked or dye-coupled lipids to study the differential uptake and use of different lipid species in the conceptus TE $[480,481]$. These results could clarify if there are specific lipid requirements by the conceptus which may be rate-limiting substrate in in vitro conditions. While lipids are best known for their role as structural components in cell and organelle membranes, they are also intricately involved in cellular metabolism and cell signaling pathways [482]. For example, PI phospholipids, one class of phospholipids elevated in the uterine lumen of sheep, is a component of cell membranes and acts as a second messenger in GPCR signaling cascades $[483,484]$. Ceramides are another class of cell-membrane lipids that regulate cell senescence, differentiation, apoptosis, and cell-cycle arrest processes and are elevated in the ULF of pregnant ewes $[408,485]$. The uptake 
of PI phospholipids or ceramides by the conceptus could provide structural substrate for cell proliferation and also enhance the ability of the conceptus to respond to its surroundings through regulation of cell signaling. Like lipids, various metabolites in the TCA cycle or in nucleotide synthesis are also altered in the ULF of pregnant ewes. Alpha-ketoglutarate, a TCA cycle intermediate, shows the largest pregnancy-dependent increase of all metabolites in the uterine lumen. In hypoxic conditions, increases in the alpha-ketoglutarate to citrate ratio promote the use of glutamine for fatty acid synthesis in cells [486]. Glutamine is a critical amino acid for conceptus metabolism but studies have primarily investigated its use for protein synthesis and oxidative metabolism [174, 179]. Future studies could investigate whether conceptus uptake of alpha-ketoglutarate from the ULF promotes and siphons glutamine into fatty acid synthesis pathways. This would provide useful information about lipid metabolism and substrate requirements for the embryo. Purine and pyrimidine metabolites are also present in the uterine lumen. These metabolites are used for DNA replication, RNA production, and generation of cofactors such as NADH that are critical for conceptus development [487]. Investigation of purine and pyrimidine metabolite uptake by the conceptus would be an interesting topic for future study as well. Overall, these results emphasize the need for more holistic approaches to understanding conceptus metabolism beyond glucose and amino acid metabolism which would provide crucial information on early embryo developmental processes.

Like other classes of lipids, PGs are higher in the uterine lumen of pregnant ewes compared to cyclic ewes. Both the endometrium and conceptus secrete 
substantial quantities of PGs during early pregnancy, which upregulate elongationand implantation- related genes in the endometrium to promote conceptus elongation [214, 217, 225]. Inhibition of PG synthesis by PTGS2, the dominant cyclooxygenase enzyme in both the conceptus and endometrium, prevents conceptus elongation and results in pregnancy loss [215, 216, 220]. However, recent studies in other species have suggested that embryonic production of PGs is not required for early pregnancy success [229, 231, 232]. In Chapter Five, CRISPR-Cas9 technology was used to determine if conceptus-derived PGs are required for conceptus survival and elongation in sheep. Inactivation of PTGS2 in the ovine conceptus did not affect TE elongation or IFNT production. Ewes who received wildtype versus PTGS2 inactivated conceptuses had similar amounts of PGs in their uterine lumen, likely due to increases in the PG synthetic capacity of the underlying pregnant endometrium by IFNT [219]. Further, gene expression of several PG regulated genes was not different between ewes. These results clarify the importance of endometrial versus embryonic derived PGs in the ULF and support the hypothesis that conceptus-derived PGs alter the uterine environment but are not essential for conceptus elongation, development or survival through day 14.

Many experiments have focused on the effects of PGs on endometrial gene expression and epithelial secretions, but it is still unclear what roles PGs play on the conceptus itself. The conceptus expresses the classical membrane bound PG receptors and the nuclear receptors PPARD and PPARG [72]. Inactivation of PPARG in ovine conceptuses compromised embryo development and suggests 
that PGs or other PPARG ligands regulate conceptus elongation directly, possibly through induction of FADS and FATP [222]. Future studies could determine how PGs regulate critical cellular processes in the conceptus, such as proliferation, migration, or adhesion, through PPARG or membrane bound receptors. These studies would clarify how endometrial PGs regulate conceptus development and, most importantly, could eventually be used to understand the threshold amount of PGs required for embryo development and determine if insufficient PG production by the endometrium is an underlying cause of pregnancy loss in ruminants.

One important question that remains is how lipids are mobilized or transported within the ULF due to their hydrophobic nature. Lipoproteins have been hypothesized to be key transporters of lipids within the ULF in sheep and cattle [160, 161]. As described in Chapter Four, cholesteryl esters and phospholipids are the most abundant lipids in the ULF and are also the predominant lipids in HDL [259]. Furthermore, APOA1, the lipid-stabilizing protein that causes lipid efflux from peripheral tissues and forms HDL, is elevated in the ULF of pregnant ewes due to production by the conceptus $[73,259,288]$. Chapter Six tested the hypothesis that APOA1 production by the conceptus causes efflux of endometrial lipids into the ULF to support elongation, implantation, and placentation processes. However, infusion of APOA1 protein into the uterine lumen of cyclic ewes did not increase any lipids in the uterine lumen, alter epithelial lipid droplets, or change gene expression in the endometrium. While APOA1 secretion by the conceptus does not increase lipids within the uterine lumen, it may mediate lipid uptake by the conceptus and affect important downstream processes 
including conceptus elongation, implantation, or the initial stages of placentation. However, APOA1 deleted conceptuses survive through day 27 of gestation and are developmentally similar to their wildtype counterparts. Collectively, these studies suggest that conceptus-derived APOA1 does not mobilize lipids into the ULF and does not play key roles in conceptus development or survival through day 27.

Although APOA1 infusion did not alter total lipid content in the ULF, it is still possible that lipids within the uterine lumen are present as lipoproteins. Future studies should characterize and compare the different classes of lipoproteins in the ULF during early pregnancy and investigate whether they are an important component of the uterine environment. This could help determine how the embryo acquires lipids to support its growth and could determine if insufficient transportation of lipoproteins into the ULF can lead to pregnancy loss. As both the conceptus and the endometrium produce APOA1 protein, it is possible that APOA1, and by inference, HDL, is required for conceptus development and growth and that endometrial production is sufficient for developmental processes through day $27[73,140,287,288]$. Infusion of morpholinos targeting APOA1 could inhibit both endometrial and conceptus production of APOA1 and investigate if it is required early in gestation. Further, APOA1 may have roles in pregnancy after day 27. For example, APOA1 and HDL are known to regulate vasodilation so it is possible that they contribute to vascularization of the placenta, processes that become more critical later in gestation [270]. Further experiments investigating this 
could answer crucial questions about the potential roles lipoproteins play in pregnancy.

Overall, the results from this dissertation highlight the complex and dynamic composition of the ULF during a crucial period for embryo development. Lipids, PGs, and lipid-associated macromolecules such as EV and lipoproteins are altered in the pregnant uterus and likely play important biological roles in early pregnancy events. As both the endometrium and conceptus secrete and internalize factors from the ULF, understanding the molecular crosstalk and the directionality of substances within the uterine lumen remains a major challenge. Future studies should expand on the present dissertation and characterize the kinetics of ULF turnover during early pregnancy. Future work could utilize in vitro culture techniques to characterize conceptus secretion of the biomolecules examined in this dissertation and superimpose those findings on the present data. Additionally, fluorescently marked molecules could be employed to quantify and track the kinetics of molecule uptake and turnover by the endometrium and conceptus. It is also still unclear how specific lipids are mobilized and transported within the uterine lumen of pregnant ewes. EVs are depleted in the ULF of pregnant ewes and thus don't account for the increases in specific lipid subpopulations in the pregnant ULF and conceptus production of APOA1 did not cause lipid efflux into the ULF. One possible explanation that has not been explored is that the endometrium secretes epithelial lipid droplets directly into the uterine lumen. This occurs in mammary epithelial cells and would explain the decrease of lipid droplets within the endometrial epithelium of pregnant ewes $[162,163]$. In support of this hypothesis, 
specific protein markers of lipid droplets have been found within the uterine lumen of cattle [74]. Alternatively, since IFNT increases PGs, proteins, and other metabolites in the ULF, future studies could test whether IFNT infusion into cyclic sheep causes mobilization of lipids into the uterine lumen [55].

Overall, the uterine environment is essential for and regulates early embryo development. Understanding the components present within the uterine lumen during the peri-implantation period is essential to understand what a conducive uterine environment is and why pregnancy loss at this stage may occur. In the past, research into lipids in the ULF has lagged that of proteins and nucleic acids due to slower advances in lipidomic technologies and the amphipathic nature of lipids. However, the dissertation here supports the overall hypothesis that lipids and lipid-associated macromolecules are critical components of the ULF that contribute to and regulate early developmental processes and endometrial function. Further investigation and expansion of these findings will improve our understanding of the underlying molecular events that lead to pregnancy success and may provide biomarkers or therapies to improve pregnancy outcomes and reproductive efficiency in agricultural species. 


\section{REFERENCES}

1. Atkinson BA, King GJ, Amoroso EC. Development of the caruncular and intercaruncular regions in the bovine endometrium. Biol Reprod 1984; 30:763-774.

2. Lawn AM, Chiquoine AD, Amoroso EC. The development of the placenta in the sheep and goat: an electron microscope study. J Anat 1969; 105:557578.

3. Kobayashi A, Behringer RR. Developmental genetics of the female reproductive tract in mammals. Nat Rev Genet 2003; 4:969-980.

4. Bartol FF, Wiley AA, Coleman DA, Wolfe DF, Riddell MG. Ovine uterine morphogenesis: effects of age and progestin administration and withdrawal on neonatal endometrial development and DNA synthesis. J Anim Sci 1988; 66:3000-3009.

5. Bartol FF, Wiley AA, Goodlett DR. Ovine uterine morphogenesis: histochemical aspects of endometrial development in the fetus and neonate. J Anim Sci 1988; 66:1303-1313.

6. Spencer TE, Kelleher AM, Bartol FF. Development and Function of Uterine Glands in Domestic Animals. Annu Rev Anim Biosci 2019; 7:125-147.

7. Wiley AA, Bartol FF, Barron DH. Histogenesis of the ovine uterus. J Anim Sci 1987; 64:1262-1269.

8. Plant TM. 60 YEARS OF NEUROENDOCRINOLOGY: The hypothalamopituitary-gonadal axis. J Endocrinol 2015; 226:T41-54.

9. Stocco C, Telleria C, Gibori G. The molecular control of corpus luteum formation, function, and regression. Endocr Rev 2007; 28:117-149.

10. Spencer TE, Bazer FW. Temporal and spatial alterations in uterine estrogen receptor and progesterone receptor gene expression during the estrous cycle and early pregnancy in the ewe. Biol Reprod 1995; 53:1527-1543.

11. Fleming JG, Spencer TE, Safe SH, Bazer FW. Estrogen regulates transcription of the ovine oxytocin receptor gene through GC-rich SP1 promoter elements. Endocrinology 2006; 147:899-911.

12. McCracken JA. Hormone receptor control of prostaglandin F2 alpha secretion by the ovine uterus. Adv Prostaglandin Thromboxane Res 1980; 8:1329-1344.

13. Roberts JS, McCracken JA, Gavagan JE, Soloff MS. Oxytocin-stimulated release of prostaglandin F2alpha from ovine endometrium in vitro: correlation with estrous cycle and oxytocin-receptor binding. Endocrinology 1976; 99:1107-1114.

14. Wathes DC, Lamming GE. The oxytocin receptor, luteolysis and the maintenance of pregnancy. J Reprod Fertil Suppl 1995; 49:53-67.

15. McCracken JA, Glew ME, Scaramuzzi RJ. Corpus luteum regression induced by prostagland in F2-alpha. J Clin Endocrinol Metab 1970; 30:544546.

16. McCracken JA, Custer EE, Lamsa JC. Luteolysis: a neuroendocrinemediated event. Physiol Rev 1999; 79:263-323. 
17. Li L, Lu X, Dean J. The maternal to zygotic transition in mammals. Mol Aspects Med 2013; 34:919-938.

18. Zernicka-Goetz M, Morris SA, Bruce AW. Making a firm decision: multifaceted regulation of cell fate in the early mouse embryo. Nat Rev Genet 2009; 10:467-477.

19. Guillomot M. Cellular interactions during implantation in domestic ruminants. J Reprod Fertil Suppl 1995; 49:39-51.

20. Wintenberger-Torres S, Flechon JE. Ultrastructural evolution of the trophoblast cells of the pre-implantation sheep blastocyst from day 8 to day 18. J Anat 1974; 118:143-153.

21. Wales RG, Cuneo CL. Morphology and chemical analysis of the sheep conceptus from the 13th to the 19th day of pregnancy. Reprod Fertil Dev 1989; 1:31-39.

22. Wooding FB, Staples LD, Peacock MA. Structure of trophoblast papillae on the sheep conceptus at implantation. J Anat 1982; 134:507-516.

23. Gray CA, Adelson DL, Bazer FW, Burghardt RC, Meeusen EN, Spencer TE. Discovery and characterization of an epithelial-specific galectin in the endometrium that forms crystals in the trophectoderm. Proc Natl Acad Sci U S A 2004; 101:7982-7987.

24. Erikson DW, Burghardt RC, Bayless KJ, Johnson GA. Secreted phosphoprotein 1 (SPP1, osteopontin) binds to integrin alpha $v$ beta 6 on porcine trophectoderm cells and integrin alpha $v$ beta 3 on uterine luminal epithelial cells, and promotes trophectoderm cell adhesion and migration. Biol Reprod 2009; 81:814-825.

25. Spencer TE, Bartol FF, Bazer FW, Johnson GA, Joyce MM. Identification and characterization of glycosylation-dependent cell adhesion molecule 1like protein expression in the ovine uterus. Biol Reprod 1999; 60:241-250.

26. Burghardt RC, Burghardt JR, Taylor JD, 2nd, Reeder AT, Nguen BT, Spencer TE, Bayless KJ, Johnson GA. Enhanced focal adhesion assembly reflects increased mechanosensation and mechanotransduction at maternal-conceptus interface and uterine wall during ovine pregnancy. Reproduction 2009; 137:567-582.

27. Frank JW, Seo H, Burghardt RC, Bayless KJ, Johnson GA. ITGAV (alpha v integrins) bind SPP1 (osteopontin) to support trophoblast cell adhesion. Reproduction 2017; 153:695-706.

28. Spencer TE, Burghardt RC, Johnson GA, Bazer FW. Conceptus signals for establishment and maintenance of pregnancy. Anim Reprod Sci 2004; 8283:537-550.

29. Guillomot M, Turbe A, Hue I, Renard JP. Staging of ovine embryos and expression of the T-box genes Brachyury and Eomesodermin around gastrulation. Reproduction 2004; 127:491-501.

30. Maddox-Hyttel P, Alexopoulos NI, Vajta G, Lewis I, Rogers P, Cann L, Callesen $\mathrm{H}$, Tveden-Nyborg $\mathrm{P}$, Trounson A. Immunohistochemical and ultrastructural characterization of the initial post-hatching development of bovine embryos. Reproduction 2003; 125:607-623. 
31. Boshier DP. A histological and histochemical examination of implantation and early placentome formation in sheep. J Reprod Fertil 1969; 19:51-61.

32. Seo H, Bazer FW, Burghardt RC, Johnson GA. Immunohistochemical Examination of Trophoblast Syncytialization during Early Placentation in Sheep. Int J Mol Sci 2019; 20.

33. Wooding FB. Electron microscopic localization of binucleate cells in the sheep placenta using phosphotungstic acid. Biol Reprod 1980; 22:357-365.

34. Wooding FB, Flint AP, Heap RB, Hobbs T. Autoradiographic evidence for migration and fusion of cells in the sheep placenta: resolution of a problem in placental classification. Cell Biol Int Rep 1981; 5:821-827.

35. Vonnahme KA, Arndt WJ, Johnson ML, Borowicz PP, Reynolds LP. Effect of morphology on placentome size, vascularity, and vasoreactivity in late pregnant sheep. Biol Reprod 2008; 79:976-982.

36. Borowicz PP, Arnold DR, Johnson ML, Grazul-Bilska AT, Redmer DA, Reynolds LP. Placental growth throughout the last two thirds of pregnancy in sheep: vascular development and angiogenic factor expression. Biol Reprod 2007; 76:259-267.

37. Imakawa K, Anthony RV, Kazemi M, Marotti KR, Polites HG, Roberts RM. Interferon-like sequence of ovine trophoblast protein secreted by embryonic trophectoderm. Nature 1987; 330:377-379.

38. Roberts RM. Interferon-tau. Nature 1993; 362:583.

39. Thatcher WW, Hansen PJ, Gross TS, Helmer SD, Plante C, Bazer FW. Antiluteolytic effects of bovine trophoblast protein-1. J Reprod Fertil Suppl 1989; 37:91-99.

40. Li J, Roberts RM. Interferon-tau and interferon-alpha interact with the same receptors in bovine endometrium. Use of a readily iodinatable form of recombinant interferon-tau for binding studies. J Biol Chem 1994; 269:13544-13550.

41. Roberts RM. A role for interferons in early pregnancy. Bioessays 1991; 13:121-126.

42. Hernandez-Ledezma JJ, Sikes JD, Murphy CN, Watson AJ, Schultz GA, Roberts RM. Expression of bovine trophoblast interferon in conceptuses derived by in vitro techniques. Biol Reprod 1992; 47:374-380.

43. Godkin JD, Bazer FW, Moffatt J, Sessions F, Roberts RM. Purification and properties of a major, low molecular weight protein released by the trophoblast of sheep blastocysts at day 13-21. J Reprod Fertil 1982; 65:141150.

44. Spencer TE, Becker WC, George P, Mirando MA, Ogle TF, Bazer FW. Ovine interferon-tau inhibits estrogen receptor up-regulation and estrogeninduced luteolysis in cyclic ewes. Endocrinology 1995; 136:4932-4944.

45. Spencer TE, Becker WC, George P, Mirando MA, Ogle TF, Bazer FW. Ovine interferon-tau regulates expression of endometrial receptors for estrogen and oxytocin but not progesterone. Biol Reprod 1995; 53:732-745.

46. Song G, Satterfield MC, Kim J, Bazer FW, Spencer TE. Gastrin-releasing peptide (GRP) in the ovine uterus: regulation by interferon tau and progesterone. Biol Reprod 2008; 79:376-386. 
47. Clemente M, de La Fuente J, Fair T, Al Naib A, Gutierrez-Adan A, Roche JF, Rizos D, Lonergan P. Progesterone and conceptus elongation in cattle: a direct effect on the embryo or an indirect effect via the endometrium? Reproduction 2009; 138:507-517.

48. Forde N, Beltman ME, Duffy GB, Duffy P, Mehta JP, O'Gaora P, Roche JF, Lonergan $\mathrm{P}$, Crowe MA. Changes in the endometrial transcriptome during the bovine estrous cycle: effect of low circulating progesterone and consequences for conceptus elongation. Biol Reprod 2011; 84:266-278.

49. Satterfield MC, Song G, Kochan KJ, Riggs PK, Simmons RM, Elsik CG, Adelson DL, Bazer FW, Zhou H, Spencer TE. Discovery of candidate genes and pathways in the endometrium regulating ovine blastocyst growth and conceptus elongation. Physiol Genomics 2009; 39:85-99.

50. Simmons RM, Satterfield MC, Welsh TH, Jr., Bazer FW, Spencer TE. HSD11B1, HSD11B2, PTGS2, and NR3C1 expression in the periimplantation ovine uterus: effects of pregnancy, progesterone, and interferon tau. Biol Reprod 2010; 82:35-43.

51. Johnson GA, Spencer TE, Burghardt RC, Taylor KM, Gray CA, Bazer FW. Progesterone modulation of osteopontin gene expression in the ovine uterus. Biol Reprod 2000; 62:1315-1321.

52. Satterfield MC, Dunlap KA, Hayashi K, Burghardt RC, Spencer TE, Bazer FW. Tight and adherens junctions in the ovine uterus: differential regulation by pregnancy and progesterone. Endocrinology 2007; 148:3922-3931.

53. Spencer TE, Ott TL, Bazer FW. Expression of interferon regulatory factors one and two in the ovine endometrium: effects of pregnancy and ovine interferon tau. Biol Reprod 1998; 58:1154-1162.

54. Choi Y, Johnson GA, Burghardt RC, Berghman LR, Joyce MM, Taylor KM, Stewart MD, Bazer FW, Spencer TE. Interferon regulatory factor-two restricts expression of interferon-stimulated genes to the endometrial stroma and glandular epithelium of the ovine uterus. Biol Reprod 2001; 65:1038-1049.

55. Brooks K, Burns G, Spencer TE. Conceptus elongation in ruminants: roles of progesterone, prostaglandin, interferon tau and cortisol. J Anim Sci Biotechnol 2014; 5:53.

56. Satterfield MC, Bazer FW, Spencer TE. Progesterone regulation of preimplantation conceptus growth and galectin 15 (LGALS15) in the ovine uterus. Biol Reprod 2006; 75:289-296.

57. Song G, Spencer TE, Bazer FW. Cathepsins in the ovine uterus: regulation by pregnancy, progesterone, and interferon tau. Endocrinology 2005; 146:4825-4833.

58. Johnson GA, Spencer TE, Hansen TR, Austin KJ, Burghardt RC, Bazer FW. Expression of the interferon tau inducible ubiquitin cross-reactive protein in the ovine uterus. Biol Reprod 1999; 61:312-318.

59. Joyce MM, White FJ, Burghardt RC, Muniz JJ, Spencer TE, Bazer FW, Johnson GA. Interferon stimulated gene 15 conjugates to endometrial cytosolic proteins and is expressed at the uterine-placental interface throughout pregnancy in sheep. Endocrinology 2005; 146:675-684. 
60. Johnson GA, Stewart MD, Gray CA, Choi Y, Burghardt RC, Yu-Lee LY, Bazer FW, Spencer TE. Effects of the estrous cycle, pregnancy, and interferon tau on 2',5'-oligoadenylate synthetase expression in the ovine uterus. Biol Reprod 2001; 64:1392-1399.

61. Ott TL, Yin J, Wiley AA, Kim HT, Gerami-Naini B, Spencer TE, Bartol FF, Burghardt RC, Bazer FW. Effects of the estrous cycle and early pregnancy on uterine expression of Mx protein in sheep (Ovis aries). Biol Reprod 1998; 59:784-794.

62. Stewart MD, Johnson GA, Bazer FW, Spencer TE. Interferon-tau (IFNtau) regulation of IFN-stimulated gene expression in cell lines lacking specific IFN-signaling components. Endocrinology 2001; 142:1786-1794.

63. Stewart MD, Choi Y, Johnson GA, Yu-Lee LY, Bazer FW, Spencer TE. Roles of Stat1, Stat2, and interferon regulatory factor-9 (IRF-9) in interferon tau regulation of IRF-1. Biol Reprod 2002; 66:393-400.

64. Flechon JE, Guillomot M, Charlier M, Flechon B, Martal J. Experimental studies on the elongation of the ewe blastocyst. Reprod Nutr Dev 1986; 26:1017-1024.

65. Alexopoulos NI, Vajta G, Maddox-Hyttel P, French AJ, Trounson AO. Stereomicroscopic and histological examination of bovine embryos following extended in vitro culture. Reprod Fertil Dev 2005; 17:799-808.

66. Spencer TE, Gray CA. Sheep uterine gland knockout (UGKO) model. Methods Mol Med 2006; 121:85-94.

67. Gray CA, Burghardt RC, Johnson GA, Bazer FW, Spencer TE. Evidence that absence of endometrial gland secretions in uterine gland knockout ewes compromises conceptus survival and elongation. Reproduction 2002; 124:289-300.

68. Gray CA, Taylor KM, Ramsey WS, Hill JR, Bazer FW, Bartol FF, Spencer TE. Endometrial glands are required for preimplantation conceptus elongation and survival. Biol Reprod 2001; 64:1608-1613.

69. Dorniak P, Welsh TH, Jr., Bazer FW, Spencer TE. Endometrial HSD11B1 and cortisol regeneration in the ovine uterus: effects of pregnancy, interferon tau, and prostaglandins. Biol Reprod 2012; 86:124.

70. Bazer FW, Wu G, Johnson GA, Kim J, Song G. Uterine histotroph and conceptus development: select nutrients and secreted phosphoprotein 1 affect mechanistic target of rapamycin cell signaling in ewes. Biol Reprod 2011; 85:1094-1107.

71. Spencer TE, Bazer FW. Uterine and placental factors regulating conceptus growth in domestic animals. J Anim Sci 2004; 82 E-Suppl:E4-13.

72. Brooks K, Burns GW, Moraes JG, Spencer TE. Analysis of the Uterine Epithelial and Conceptus Transcriptome and Luminal Fluid Proteome During the Peri-Implantation Period of Pregnancy in Sheep. Biol Reprod 2016; 95:88.

73. Koch JM, Ramadoss J, Magness RR. Proteomic profile of uterine luminal fluid from early pregnant ewes. J Proteome Res 2010; 9:3878-3885.

74. Forde N, Mehta JP, McGettigan PA, Mamo S, Bazer FW, Spencer TE, Lonergan P. Alterations in expression of endometrial genes coding for 
proteins secreted into the uterine lumen during conceptus elongation in cattle. BMC Genomics 2013; 14:321.

75. Romero JJ, Liebig BE, Broeckling CD, Prenni JE, Hansen TR. Pregnancyinduced changes in metabolome and proteome in ovine uterine flushings. Biol Reprod 2017; 97:273-287.

76. Gould SJ, Raposo G. As we wait: coping with an imperfect nomenclature for extracellular vesicles. J Extracell Vesicles 2013; 2.

77. Thery C. Exosomes: secreted vesicles and intercellular communications. F1000 Biol Rep 2011; 3:15.

78. Pan BT, Teng K, Wu C, Adam M, Johnstone RM. Electron microscopic evidence for externalization of the transferrin receptor in vesicular form in sheep reticulocytes. J Cell Biol 1985; 101:942-948.

79. Harding C, Heuser J, Stahl P. Receptor-mediated endocytosis of transferrin and recycling of the transferrin receptor in rat reticulocytes. J Cell Biol 1983; 97:329-339.

80. Raposo G, Nijman HW, Stoorvogel W, Liejendekker R, Harding CV, Melief CJ, Geuze HJ. B lymphocytes secrete antigen-presenting vesicles. J Exp Med 1996; 183:1161-1172.

81. Villarroya-Beltri C, Baixauli F, Mittelbrunn M, Fernandez-Delgado I, Torralba D, Moreno-Gonzalo O, Baldanta S, Enrich C, Guerra S, SanchezMadrid F. ISGylation controls exosome secretion by promoting lysosomal degradation of MVB proteins. Nat Commun 2016; 7:13588.

82. Villarroya-Beltri C, Gutierrez-Vazquez C, Sanchez-Cabo F, PerezHernandez D, Vazquez J, Martin-Cofreces N, Martinez-Herrera DJ, Pascual-Montano A, Mittelbrunn M, Sanchez-Madrid F. Sumoylated hnRNPA2B1 controls the sorting of miRNAs into exosomes through binding to specific motifs. Nat Commun 2013; 4:2980.

83. Baietti MF, Zhang Z, Mortier E, Melchior A, Degeest G, Geeraerts A, Ivarsson Y, Depoortere F, Coomans C, Vermeiren E, Zimmermann P, David G. Syndecan-syntenin-ALIX regulates the biogenesis of exosomes. Nat Cell Biol 2012; 14:677-685.

84. Trajkovic K, Hsu C, Chiantia S, Rajendran L, Wenzel D, Wieland F, Schwille $\mathrm{P}$, Brugger B, Simons M. Ceramide triggers budding of exosome vesicles into multivesicular endosomes. Science 2008; 319:1244-1247.

85. Ostrowski M, Carmo NB, Krumeich S, Fanget I, Raposo G, Savina A, Moita CF, Schauer K, Hume AN, Freitas RP, Goud B, Benaroch P, et al. Rab27a and Rab27b control different steps of the exosome secretion pathway. Nat Cell Biol 2010; 12:19-30; sup pp 11-13.

86. Wei Y, Wang D, Jin F, Bian Z, Li L, Liang H, Li M, Shi L, Pan C, Zhu D, Chen X, Hu G, et al. Pyruvate kinase type M2 promotes tumour cell exosome release via phosphorylating synaptosome-associated protein 23. Nat Commun 2017; 8:14041.

87. Muralidharan-Chari V, Clancy JW, Sedgwick A, D'Souza-Schorey C. Microvesicles: mediators of extracellular communication during cancer progression. J Cell Sci 2010; 123:1603-1611. 
88. Tricarico C, Clancy J, D'Souza-Schorey C. Biology and biogenesis of shed microvesicles. Small GTPases 2017; 8:220-232.

89. McBride JD, Rodriguez-Menocal L, Badiavas EV. Extracellular Vesicles as Biomarkers and Therapeutics in Dermatology: A Focus on Exosomes. $J$ Invest Dermatol 2017; 137:1622-1629.

90. Ageta H, Ageta-Ishihara N, Hitachi K, Karayel O, Onouchi T, Yamaguchi H, Kahyo T, Hatanaka K, Ikegami K, Yoshioka Y, Nakamura K, Kosaka N, et al. UBL3 modification influences protein sorting to small extracellular vesicles. Nat Commun 2018; 9:3936.

91. Cha DJ, Franklin JL, Dou Y, Liu Q, Higginbotham JN, Demory Beckler M, Weaver AM, Vickers K, Prasad N, Levy S, Zhang B, Coffey RJ, et al. KRASdependent sorting of miRNA to exosomes. Elife 2015; 4:e07197.

92. Squadrito ML, Baer C, Burdet F, Maderna C, Gilfillan GD, Lyle R, Ibberson $\mathrm{M}$, De Palma M. Endogenous RNAs modulate microRNA sorting to exosomes and transfer to acceptor cells. Cell Rep 2014; 8:1432-1446.

93. Parolini I, Federici C, Raggi C, Lugini L, Palleschi S, De Milito A, Coscia C, lessi E, Logozzi M, Molinari A, Colone M, Tatti M, et al. Microenvironmental $\mathrm{pH}$ is a key factor for exosome traffic in tumor cells. J Biol Chem 2009; 284:34211-34222.

94. Kucharzewska P, Belting M. Emerging roles of extracellular vesicles in the adaptive response of tumour cells to microenvironmental stress. J Extracell Vesicles 2013; 2.

95. Mittelbrunn M, Gutierrez-Vazquez C, Villarroya-Beltri C, Gonzalez S, Sanchez-Cabo F, Gonzalez MA, Bernad A, Sanchez-Madrid F. Unidirectional transfer of microRNA-loaded exosomes from $T$ cells to antigen-presenting cells. Nat Commun 2011; 2:282.

96. Laulagnier K, Motta C, Hamdi S, Roy S, Fauvelle F, Pageaux JF, Kobayashi T, Salles JP, Perret B, Bonnerot C, Record M. Mast cell- and dendritic cellderived exosomes display a specific lipid composition and an unusual membrane organization. Biochem J 2004; 380:161-171.

97. Skotland T, Hessvik NP, Sandvig K, Llorente A. Exosomal lipid composition and the role of ether lipids and phosphoinositides in exosome biology. $J$ Lipid Res 2019; 60:9-18.

98. Record M, Silvente-Poirot S, Poirot M, Wakelam MJO. Extracellular vesicles: lipids as key components of their biogenesis and functions. J Lipid Res 2018; 59:1316-1324.

99. Subra C, Grand D, Laulagnier K, Stella A, Lambeau G, Paillasse M, De Medina P, Monsarrat B, Perret B, Silvente-Poirot S, Poirot M, Record M. Exosomes account for vesicle-mediated transcellular transport of activatable phospholipases and prostaglandins. J Lipid Res 2010; 51:21052120.

100. Valadi H, Ekstrom K, Bossios A, Sjostrand M, Lee JJ, Lotvall JO. Exosomemediated transfer of mRNAs and microRNAs is a novel mechanism of genetic exchange between cells. Nat Cell Biol 2007; 9:654-659. 
101. Kosaka N, Iguchi H, Yoshioka Y, Takeshita F, Matsuki Y, Ochiya T. Secretory mechanisms and intercellular transfer of microRNAs in living cells. J Biol Chem 2010; 285:17442-17452.

102. Rosa-Fernandes L, Rocha VB, Carregari VC, Urbani A, Palmisano G. A Perspective on Extracellular Vesicles Proteomics. Front Chem 2017; 5:102.

103. Kowal J, Arras G, Colombo M, Jouve M, Morath JP, Primdal-Bengtson B, Dingli F, Loew D, Tkach M, Thery C. Proteomic comparison defines novel markers to characterize heterogeneous populations of extracellular vesicle subtypes. Proc Natl Acad Sci U S A 2016; 113:E968-977.

104. Somiya M. Where does the cargo go?: Solutions to provide experimental support for the "extracellular vesicle cargo transfer hypothesis". J Cell Commun Signal 2020; 14:135-146.

105. Simpson RJ, Jensen SS, Lim JW. Proteomic profiling of exosomes: current perspectives. Proteomics 2008; 8:4083-4099.

106. Keller S, Ridinger J, Rupp AK, Janssen JW, Altevogt P. Body fluid derived exosomes as a novel template for clinical diagnostics. J Transl Med 2011; 9:86.

107. Mulcahy LA, Pink RC, Carter DR. Routes and mechanisms of extracellular vesicle uptake. J Extracell Vesicles 2014; 3.

108. Atay S, Gercel-Taylor C, Taylor DD. Human trophoblast-derived exosomal fibronectin induces pro-inflammatory IL-1beta production by macrophages. Am J Reprod Immunol 2011; 66:259-269.

109. Rana S, Claas C, Kretz CC, Nazarenko I, Zoeller M. Activation-induced internalization differs for the tetraspanins CD9 and Tspan8: Impact on tumor cell motility. Int J Biochem Cell Biol 2011; 43:106-119.

110. Nazarenko I, Rana S, Baumann A, McAlear J, Hellwig A, Trendelenburg M, Lochnit G, Preissner KT, Zoller M. Cell surface tetraspanin Tspan8 contributes to molecular pathways of exosome-induced endothelial cell activation. Cancer Res 2010; 70:1668-1678.

111. Escrevente C, Keller S, Altevogt P, Costa J. Interaction and uptake of exosomes by ovarian cancer cells. BMC Cancer 2011; 11:108.

112. Feng $D$, Zhao WL, Ye YY, Bai $X C$, Liu RQ, Chang LF, Zhou Q, Sui SF. Cellular internalization of exosomes occurs through phagocytosis. Traffic 2010; 11:675-687.

113. Morelli AE, Larregina AT, Shufesky WJ, Sullivan ML, Stolz DB, Papworth GD, Zahorchak AF, Logar AJ, Wang Z, Watkins SC, Falo LD, Jr., Thomson AW. Endocytosis, intracellular sorting, and processing of exosomes by dendritic cells. Blood 2004; 104:3257-3266.

114. Ferraz M, Carothers A, Dahal R, Noonan MJ, Songsasen N. Oviductal extracellular vesicles interact with the spermatozoon's head and mid-piece and improves its motility and fertilizing ability in the domestic cat. Sci Rep 2019; 9:9484.

115. Al-Dossary AA, Strehler EE, Martin-Deleon PA. Expression and secretion of plasma membrane Ca2+-ATPase 4a (PMCA4a) during murine estrus: association with oviductal exosomes and uptake in sperm. PLoS One 2013; 8:e80181. 
116. Alminana C, Corbin E, Tsikis G, Alcantara-Neto AS, Labas V, Reynaud K, Galio L, Uzbekov R, Garanina AS, Druart X, Mermillod P. Oviduct extracellular vesicles protein content and their role during oviduct-embryo cross-talk. Reproduction 2017; 154:153-168.

117. Lopera-Vasquez R, Hamdi M, Fernandez-Fuertes B, Maillo V, BeltranBrena P, Calle A, Redruello A, Lopez-Martin S, Gutierrez-Adan A, YanezMo M, Ramirez MA, Rizos D. Extracellular Vesicles from BOEC in In Vitro Embryo Development and Quality. PLoS One 2016; 11:e0148083.

118. Burns GW, Brooks KE, Spencer TE. Extracellular Vesicles Originate from the Conceptus and Uterus During Early Pregnancy in Sheep. Biol Reprod 2016; 94:56.

119. Burns G, Brooks K, Wildung M, Navakanitworakul R, Christenson LK, Spencer TE. Extracellular vesicles in luminal fluid of the ovine uterus. PLoS One 2014; $9:$ e90913.

120. Ruiz-Gonzalez I, Xu J, Wang X, Burghardt RC, Dunlap KA, Bazer FW. Exosomes, endogenous retroviruses and toll-like receptors: pregnancy recognition in ewes. Reproduction 2015; 149:281-291.

121. Bidarimath M, Khalaj K, Kridli RT, Kan FW, Koti M, Tayade C. Extracellular vesicle mediated intercellular communication at the porcine maternal-fetal interface: A new paradigm for conceptus-endometrial cross-talk. Sci Rep 2017; 7:40476.

122. Liu WM, Cheng RR, Niu ZR, Chen AC, Ma MY, Li T, Chiu PC, Pang RT, Lee YL, Ou JP, Yao YQ, Yeung WSB. Let-7 derived from endometrial extracellular vesicles is an important inducer of embryonic diapause in mice. Sci Adv 2020; 6.

123. Nakamura K, Kusama K, Bai R, Sakurai T, Isuzugawa K, Godkin JD, Suda Y, Imakawa K. Induction of IFNT-Stimulated Genes by Conceptus-Derived Exosomes during the Attachment Period. PLoS One 2016; 11:e0158278.

124. $\mathrm{Ng} \mathrm{YH,} \mathrm{Rome} \mathrm{S,} \mathrm{Jalabert} \mathrm{A,} \mathrm{Forterre} \mathrm{A,} \mathrm{Singh} \mathrm{H,} \mathrm{Hincks} \mathrm{CL,} \mathrm{Salamonsen}$ LA. Endometrial exosomes/microvesicles in the uterine microenvironment: a new paradigm for embryo-endometrial cross talk at implantation. PLoS One 2013; 8:e58502.

125. O'Neil EV, Burns GW, Spencer TE. Extracellular vesicles: Novel regulators of conceptus-uterine interactions? Theriogenology 2020; 150:106-112.

126. Burns GW, Brooks KE, O'Neil EV, Hagen DE, Behura SK, Spencer TE. Progesterone effects on extracellular vesicles in the sheep uterus. Biol Reprod 2018; 98:612-622.

127. Giacomini E, Vago R, Sanchez AM, Podini P, Zarovni N, Murdica V, Rizzo $R$, Bortolotti D, Candiani M, Vigano P. Secretome of in vitro cultured human embryos contains extracellular vesicles that are uptaken by the maternal side. Sci Rep 2017; 7:5210.

128. Sheller-Miller S, Lei J, Saade G, Salomon C, Burd I, Menon R. FetoMaternal Trafficking of Exosomes in Murine Pregnancy Models. Front Pharmacol 2016; 7:432. 
129. Lv C, Yu WX, Wang Y, Yi DJ, Zeng MH, Xiao HM. MiR-21 in extracellular vesicles contributes to the growth of fertilized eggs and embryo development in mice. Biosci Rep 2018; 38.

130. Desrochers LM, Bordeleau F, Reinhart-King CA, Cerione RA, Antonyak MA. Microvesicles provide a mechanism for intercellular communication by embryonic stem cells during embryo implantation. Nat Commun 2016; 7:11958.

131. Evans J, Rai A, Nguyen HPT, Poh QH, Elglass K, Simpson RJ, Salamonsen LA, Greening DW. Human Endometrial Extracellular Vesicles Functionally Prepare Human Trophectoderm Model for Implantation: Understanding Bidirectional Maternal-Embryo Communication. Proteomics 2019; 19:e1800423.

132. Balaguer N, Moreno I, Herrero M, Gonzalez M, Simon C, Vilella F. Heterogeneous nuclear ribonucleoprotein C1 may control miR-30d levels in endometrial exosomes affecting early embryo implantation. Mol Hum Reprod 2018; 24:411-425.

133. Gurung S, Greening DW, Catt S, Salamonsen L, Evans J. Exosomes and soluble secretome from hormone-treated endometrial epithelial cells direct embryo implantation. Mol Hum Reprod 2020; 26:510-520.

134. Nakamura K, Kusama K, Ideta A, Imakawa K, Hori M. IFNT-independent effects of intrauterine extracellular vesicles (EVs) in cattle. Reproduction 2020; 159:503-511.

135. Kusama K, Nakamura K, Bai R, Nagaoka K, Sakurai T, Imakawa K. Intrauterine exosomes are required for bovine conceptus implantation. Biochem Biophys Res Commun 2018; 495:1370-1375.

136. Nakamura K, Kusama K, Ideta A, Kimura K, Hori M, Imakawa K. Effects of miR-98 in intrauterine extracellular vesicles on maternal immune regulation during the peri-implantation period in cattle. Sci Rep 2019; 9:20330.

137. Zhao G, Yang C, Yang J, Liu P, Jiang K, Shaukat A, Wu H, Deng G. Placental exosome-mediated Bta-miR-499-Lin28B/let-7 axis regulates inflammatory bias during early pregnancy. Cell Death Dis 2018; 9:704.

138. Gao H, Wu G, Spencer TE, Johnson GA, Li X, Bazer FW. Select nutrients in the ovine uterine lumen. I. Amino acids, glucose, and ions in uterine lumenal flushings of cyclic and pregnant ewes. Biol Reprod 2009; 80:86-93.

139. Gao H, Wu G, Spencer TE, Johnson GA, Bazer FW. Select nutrients in the ovine uterine lumen. ii. glucose transporters in the uterus and periimplantation conceptuses. Biol Reprod 2009; 80:94-104.

140. Moraes JGN, Behura SK, Geary TW, Hansen PJ, Neibergs HL, Spencer TE. Uterine influences on conceptus development in fertility-classified animals. Proc Natl Acad Sci U S A 2018; 115:E1749-E1758.

141. Murdoch RN. Glycogen, glycogen-metabolizing enzymes, and acid and alkaline phosphatases in the endometrium of the ewe during early pregnancy. Aust J Biol Sci 1970; 23:1289-1296.

142. Whyte A, Robson T. Saccharides localized by fluorescent lectins on trophectoderm and endometrium prior to implantation in pigs, sheep and equids. Placenta 1984; 5:533-540. 
143. Yang $\mathrm{Y}$, Wang L, Chen C, Qi H, Baker PN, Liu X, Zhang H, Han TL. Metabolic Changes of Maternal Uterine Fluid, Uterus, and Plasma during the Peri-implantation Period of Early Pregnancy in Mice. Reprod Sci 2020; 27:488-502.

144. Groebner AE, Rubio-Aliaga I, Schulke K, Reichenbach HD, Daniel H, Wolf $\mathrm{E}$, Meyer HH, Ulbrich SE. Increase of essential amino acids in the bovine uterine lumen during preimplantation development. Reproduction 2011; 141:685-695.

145. Gao H, Wu G, Spencer TE, Johnson GA, Bazer FW. Select nutrients in the ovine uterine lumen. III. Cationic amino acid transporters in the ovine uterus and peri-implantation conceptuses. Biol Reprod 2009; 80:602-609.

146. Gao H, Wu G, Spencer TE, Johnson GA, Bazer FW. Select nutrients in the ovine uterine lumen. IV. Expression of neutral and acidic amino acid transporters in ovine uteri and peri-implantation conceptuses. Biol Reprod 2009; 80:1196-1208.

147. Simintiras CA, Sanchez JM, McDonald M, Lonergan P. The influence of progesterone on bovine uterine fluid energy, nucleotide, vitamin, cofactor, peptide, and xenobiotic composition during the conceptus elongationinitiation window. Sci Rep 2019; 9:7716.

148. Forde N, McGettigan PA, Mehta JP, O'Hara L, Mamo S, Bazer FW, Spencer TE, Lonergan P. Proteomic analysis of uterine fluid during the preimplantation period of pregnancy in cattle. Reproduction 2014; 147:575587.

149. Pajor AM. Molecular properties of the SLC13 family of dicarboxylate and sulfate transporters. Pflugers Arch 2006; 451:597-605.

150. Moraes JGN, Behura SK, Bishop JV, Hansen TR, Geary TW, Spencer TE. Analysis of the uterine lumen in fertility-classified heifers: II. Proteins and metabolites. Biol Reprod 2020; 102:571-587.

151. Walsh SC, Miles JR, Yao L, Broeckling CD, Rempel LA, Wright-Johnson EC, Pannier AK. Metabolic compounds within the porcine uterine environment are unique to the type of conceptus present during the early stages of blastocyst elongation. Mol Reprod Dev 2020; 87:174-190.

152. Fahy E, Subramaniam S, Murphy RC, Nishijima M, Raetz CR, Shimizu T, Spener F, van Meer G, Wakelam MJ, Dennis EA. Update of the LIPID MAPS comprehensive classification system for lipids. J Lipid Res 2009; 50 Suppl:S9-14.

153. Simintiras CA, Sanchez JM, McDonald M, Lonergan P. Progesterone alters the bovine uterine fluid lipidome during the period of elongation. Reproduction 2019; 157:399-411.

154. Simintiras CA, Sanchez JM, McDonald M, Lonergan P. The biochemistry surrounding bovine conceptus elongation. Biol Reprod 2019; 101:328-337.

155. Liszewska E, Reinaud P, Billon-Denis E, Dubois O, Robin P, Charpigny G. Lysophosphatidic acid signaling during embryo development in sheep: involvement in prostaglandin synthesis. Endocrinology 2009; 150:422-434. 
156. Moraes JGN, Behura SK, Geary TW, Spencer TE. Analysis of the uterine lumen in fertility-classified heifers: I. Glucose, prostaglandins, and lipids. Biol Reprod 2020; 102:456-474.

157. Ribeiro ES, Greco LF, Bisinotto RS, Lima FS, Thatcher WW, Santos JE. Biology of Preimplantation Conceptus at the Onset of Elongation in Dairy Cows. Biol Reprod 2016; 94:97.

158. van der Vusse GJ. Albumin as fatty acid transporter. Drug Metab Pharmacokinet 2009; 24:300-307.

159. Weinberg JM. Lipotoxicity. Kidney Int 2006; 70:1560-1566.

160. Ribeiro ES. Symposium review: Lipids as regulators of conceptus development: Implications for metabolic regulation of reproduction in dairy cattle. J Dairy Sci 2018; 101:3630-3641.

161. Ribeiro ES, Santos JE, Thatcher WW. Role of lipids on elongation of the preimplantation conceptus in ruminants. Reproduction 2016; 152:R115126.

162. Boshier DP, Fairclough RJ, Holloway H. Assessment of sheep blastocyst effects on neutral lipids in the uterine caruncular epithelium. J Reprod Fertil 1987; 79:569-573.

163. Brinsfield TH, Hawk HW. Control by progesterone of the concentration of lipid droplets in epithelial cells of the sheep endometrium. J Anim Sci 1973; 36:919-922.

164. Krisher RL, Prather RS. A role for the Warburg effect in preimplantation embryo development: metabolic modification to support rapid cell proliferation. Mol Reprod Dev 2012; 79:311-320.

165. Vander Heiden MG, Cantley LC, Thompson CB. Understanding the Warburg effect: the metabolic requirements of cell proliferation. Science 2009; 324:1029-1033.

166. Warburg O. On the origin of cancer cells. Science 1956; 123:309-314.

167. Liberti MV, Locasale JW. The Warburg Effect: How Does it Benefit Cancer Cells? Trends Biochem Sci 2016; 41:211-218.

168. Smith B, Schafer XL, Ambeskovic A, Spencer CM, Land H, Munger J. Addiction to Coupling of the Warburg Effect with Glutamine Catabolism in Cancer Cells. Cell Rep 2016; 17:821-836.

169. Rieger D, Loskutoff NM, Betteridge KJ. Developmentally related changes in the uptake and metabolism of glucose, glutamine and pyruvate by cattle embryos produced in vitro. Reprod Fertil Dev 1992; 4:547-557.

170. Gardner DK, Lane M, Batt P. Uptake and metabolism of pyruvate and glucose by individual sheep preattachment embryos developed in vivo. Mol Reprod Dev 1993; 36:313-319.

171. Du ZF, Wales RG. Glycolysis and glucose oxidation by the sheep conceptus at different oxygen concentrations. Reprod Fertil Dev 1993; 5:383-393.

172. Wales RG, Du ZF. Contribution of the pentose phosphate pathway to glucose utilization by preimplantation sheep embryos. Reprod Fertil Dev 1993; 5:329-340.

173. Fischer B, Bavister BD. Oxygen tension in the oviduct and uterus of rhesus monkeys, hamsters and rabbits. J Reprod Fertil 1993; 99:673-679. 
174. Wales RG, Du ZF. The metabolism of glutamine by the preimplantation sheep conceptus and its interaction with glucose. Reprod Fertil Dev 1994; 6:659-667.

175. Liu GY, Sabatini DM. mTOR at the nexus of nutrition, growth, ageing and disease. Nat Rev Mol Cell Biol 2020; 21:183-203.

176. Shimobayashi M, Hall MN. Making new contacts: the mTOR network in metabolism and signalling crosstalk. Nat Rev Mol Cell Biol 2014; 15:155162.

177. Gangloff YG, Mueller M, Dann SG, Svoboda P, Sticker M, Spetz JF, Um $\mathrm{SH}$, Brown EJ, Cereghini S, Thomas G, Kozma SC. Disruption of the mouse mTOR gene leads to early postimplantation lethality and prohibits embryonic stem cell development. Mol Cell Biol 2004; 24:9508-9516.

178. Gao H, Wu G, Spencer TE, Johnson GA, Bazer FW. Select nutrients in the ovine uterine lumen. VI. Expression of FK506-binding protein 12-rapamycin complex-associated protein 1 (FRAP1) and regulators and effectors of mTORC1 and mTORC2 complexes in ovine uteri and conceptuses. Biol Reprod 2009; 81:87-100.

179. Kim JY, Burghardt RC, Wu G, Johnson GA, Spencer TE, Bazer FW. Select nutrients in the ovine uterine lumen. VII. Effects of arginine, leucine, glutamine, and glucose on trophectoderm cell signaling, proliferation, and migration. Biol Reprod 2011; 84:62-69.

180. Kim JY, Burghardt RC, Wu G, Johnson GA, Spencer TE, Bazer FW. Select nutrients in the ovine uterine lumen. VIII. Arginine stimulates proliferation of ovine trophectoderm cells through MTOR-RPS6K-RPS6 signaling cascade and synthesis of nitric oxide and polyamines. Biol Reprod 2011; 84:70-78.

181. Gonzalez IM, Martin PM, Burdsal C, Sloan JL, Mager S, Harris T, Sutherland $\mathrm{AE}$. Leucine and arginine regulate trophoblast motility through mTOR-dependent and independent pathways in the preimplantation mouse embryo. Dev Biol 2012; 361:286-300.

182. Kim J, Song G, Wu G, Bazer FW. Functional roles of fructose. Proc Natl Acad Sci U S A 2012; 109:E1619-1628.

183. Sponchiado M, Gonella-Diaza AM, Rocha CC, Turco EGL, Pugliesi G, Leroy J, Binelli $\mathrm{M}$. The pre-hatching bovine embryo transforms the uterine luminal metabolite composition in vivo. Sci Rep 2019; 9:8354.

184. Balsinde J, Winstead MV, Dennis EA. Phospholipase A(2) regulation of arachidonic acid mobilization. FEBS Lett 2002; 531:2-6.

185. Gijon MA, Leslie CC. Regulation of arachidonic acid release and cytosolic phospholipase A2 activation. J Leukoc Biol 1999; 65:330-336.

186. Garavito RM, Malkowski MG, DeWitt DL. The structures of prostaglandin endoperoxide $\mathrm{H}$ synthases-1 and -2. Prostaglandins Other Lipid Mediat 2002; 68-69:129-152.

187. Kiefer JR, Pawlitz JL, Moreland KT, Stegeman RA, Hood WF, Gierse JK, Stevens AM, Goodwin DC, Rowlinson SW, Marnett LJ, Stallings WC, Kurumbail RG. Structural insights into the stereochemistry of the cyclooxygenase reaction. Nature 2000; 405:97-101. 
188. Kurumbail RG, Stevens AM, Gierse JK, McDonald JJ, Stegeman RA, Pak JY, Gildehaus D, Miyashiro JM, Penning TD, Seibert K, Isakson PC, Stallings WC. Structural basis for selective inhibition of cyclooxygenase-2 by anti-inflammatory agents. Nature 1996; 384:644-648.

189. FitzGerald GA. COX-2 and beyond: Approaches to prostaglandin inhibition in human disease. Nat Rev Drug Discov 2003; 2:879-890.

190. Masferrer JL, Zweifel BS, Manning PT, Hauser SD, Leahy KM, Smith WG, Isakson PC, Seibert K. Selective inhibition of inducible cyclooxygenase 2 in vivo is antiinflammatory and nonulcerogenic. Proc Natl Acad Sci U S A 1994; 91:3228-3232.

191. Herschman HR. Function and regulation of prostaglandin synthase 2. Adv Exp Med Biol 1999; 469:3-8.

192. Rouzer CA, Marnett LJ. Cyclooxygenases: structural and functional insights. J Lipid Res 2009; 50 Suppl:S29-34.

193. Smith WL, DeWitt DL, Garavito RM. Cyclooxygenases: structural, cellular, and molecular biology. Annu Rev Biochem 2000; 69:145-182.

194. Simmons DL, Botting RM, Hla T. Cyclooxygenase isozymes: the biology of prostaglandin synthesis and inhibition. Pharmacol Rev 2004; 56:387-437.

195. Chan BS, Satriano JA, Pucci M, Schuster VL. Mechanism of prostaglandin E2 transport across the plasma membrane of HeLa cells and Xenopus oocytes expressing the prostaglandin transporter "PGT". J Biol Chem 1998; 273:6689-6697.

196. Lee J, McCracken JA, Banu SK, Rodriguez R, Nithy TK, Arosh JA. Transport of prostaglandin $\mathrm{F}$ (2alpha) pulses from the uterus to the ovary at the time of luteolysis in ruminants is regulated by prostaglandin transportermediated mechanisms. Endocrinology 2010; 151:3326-3335.

197. Folco G, Murphy RC. Eicosanoid transcellular biosynthesis: from cell-cell interactions to in vivo tissue responses. Pharmacol Rev 2006; 58:375-388.

198. Ushikubi F, Segi E, Sugimoto Y, Murata T, Matsuoka T, Kobayashi T, Hizaki H, Tuboi K, Katsuyama M, Ichikawa A, Tanaka T, Yoshida N, et al. Impaired febrile response in mice lacking the prostaglandin E receptor subtype EP3. Nature 1998; 395:281-284.

199. Murata T, Ushikubi F, Matsuoka T, Hirata M, Yamasaki A, Sugimoto $Y$, Ichikawa A, Aze Y, Tanaka T, Yoshida N, Ueno A, Oh-ishi S, et al. Altered pain perception and inflammatory response in mice lacking prostacyclin receptor. Nature 1997; 388:678-682.

200. Namba T, Sugimoto Y, Negishi M, Irie A, Ushikubi F, Kakizuka A, Ito S, Ichikawa A, Narumiya S. Alternative splicing of C-terminal tail of prostaglandin E receptor subtype EP3 determines G-protein specificity. Nature 1993; 365:166-170.

201. Dennis EA, Norris PC. Eicosanoid storm in infection and inflammation. Nat Rev Immunol 2015; 15:511-523.

202. Soberman RJ, Christmas P. The organization and consequences of eicosanoid signaling. J Clin Invest 2003; 111:1107-1113.

203. Fu J, Gaetani S, Oveisi F, Lo Verme J, Serrano A, Rodriguez De Fonseca F, Rosengarth A, Luecke H, Di Giacomo B, Tarzia G, Piomelli D. 
Oleylethanolamide regulates feeding and body weight through activation of the nuclear receptor PPAR-alpha. Nature 2003; 425:90-93.

204. Forman BM, Tontonoz P, Chen J, Brun RP, Spiegelman BM, Evans RM. 15-Deoxy-delta 12, 14-prostaglandin $\mathrm{J} 2$ is a ligand for the adipocyte determination factor PPAR gamma. Cell 1995; 83:803-812.

205. Lo Verme J, Fu J, Astarita G, La Rana G, Russo R, Calignano A, Piomelli $D$. The nuclear receptor peroxisome proliferator-activated receptor-alpha mediates the anti-inflammatory actions of palmitoylethanolamide. Mol Pharmacol 2005; 67:15-19.

206. Kliewer SA, Sundseth SS, Jones SA, Brown PJ, Wisely GB, Koble CS, Devchand P, Wahli W, Willson TM, Lenhard JM, Lehmann JM. Fatty acids and eicosanoids regulate gene expression through direct interactions with peroxisome proliferator-activated receptors alpha and gamma. Proc Natl Acad Sci U S A 1997; 94:4318-4323.

207. Nagy L, Tontonoz P, Alvarez JG, Chen H, Evans RM. Oxidized LDL regulates macrophage gene expression through ligand activation of PPARgamma. Cell 1998; 93:229-240.

208. Chandra V, Huang $P$, Hamuro $Y$, Raghuram S, Wang Y, Burris TP, Rastinejad F. Structure of the intact PPAR-gamma-RXR- nuclear receptor complex on DNA. Nature 2008; 456:350-356.

209. Tyagi S, Gupta P, Saini AS, Kaushal C, Sharma S. The peroxisome proliferator-activated receptor: A family of nuclear receptors role in various diseases. J Adv Pharm Technol Res 2011; 2:236-240.

210. Schoonjans K, Staels B, Auwerx J. Role of the peroxisome proliferatoractivated receptor (PPAR) in mediating the effects of fibrates and fatty acids on gene expression. J Lipid Res 1996; 37:907-925.

211. Ellinwood WE, Nett TM, Niswender GD. Maintenance of the corpus luteum of early pregnancy in the ewe. II. Prostaglandin secretion by the endometrium in vitro and in vivo. Biol Reprod 1979; 21:845-856.

212. Ulbrich SE, Schulke K, Groebner AE, Reichenbach HD, Angioni C, Geisslinger $\mathrm{G}$, Meyer $\mathrm{HH}$. Quantitative characterization of prostaglandins in the uterus of early pregnant cattle. Reproduction 2009; 138:371-382.

213. Downie J, Poyser NL, Wunderlich M. Levels of prostaglandins in human endometrium during the normal menstrual cycle. J Physiol 1974; 236:465472.

214. Charpigny G, Reinaud P, Tamby JP, Creminon C, Martal J, Maclouf J, Guillomot M. Expression of cyclooxygenase-1 and -2 in ovine endometrium during the estrous cycle and early pregnancy. Endocrinology 1997; 138:2163-2171.

215. Charpigny G, Reinaud P, Creminon C, Tamby JP. Correlation of increased concentration of ovine endometrial cyclooxygenase 2 with the increase in PGE2 and PGD2 in the late luteal phase. J Reprod Fertil 1999; 117:315324.

216. Dorniak P, Bazer FW, Spencer TE. Prostaglandins regulate conceptus elongation and mediate effects of interferon tau on the ovine uterine endometrium. Biol Reprod 2011; 84:1119-1127. 
217. Arosh JA, Banu SK, Chapdelaine P, Fortier MA. Temporal and tissuespecific expression of prostaglandin receptors EP2, EP3, EP4, FP, and cyclooxygenases 1 and 2 in uterus and fetal membranes during bovine pregnancy. Endocrinology 2004; 145:407-417.

218. Asselin E, Bazer FW, Fortier MA. Recombinant ovine and bovine interferons tau regulate prostaglandin production and oxytocin response in cultured bovine endometrial cells. Biol Reprod 1997; 56:402-408.

219. Kim S, Choi Y, Spencer TE, Bazer FW. Effects of the estrous cycle, pregnancy and interferon tau on expression of cyclooxygenase two (COX2) in ovine endometrium. Reprod Biol Endocrinol 2003; 1:58.

220. Charpigny G, Reinaud P, Tamby JP, Creminon C, Guillomot M. Cyclooxygenase-2 unlike cyclooxygenase-1 is highly expressed in ovine embryos during the implantation period. Biol Reprod 1997; 57:1032-1040.

221. Lewis GS, Waterman RA. Metabolism of arachidonic acid in vitro by ovine conceptuses recovered during early pregnancy. Prostaglandins 1985; 30:263-283.

222. Brooks KE, Burns GW, Spencer TE. Peroxisome proliferator activator receptor gamma (PPARG) regulates conceptus elongation in sheep. Biol Reprod 2015; 92:42.

223. Spencer TE, Forde N, Dorniak P, Hansen TR, Romero JJ, Lonergan P. Conceptus-derived prostaglandins regulate gene expression in the endometrium prior to pregnancy recognition in ruminants. Reproduction 2013; 146:377-387.

224. Lee HY, Acosta TJ, Skarzynski DJ, Okuda K. Prostaglandin F2alpha stimulates 11Beta-hydroxysteroid dehydrogenase 1 enzyme bioactivity and protein expression in bovine endometrial stromal cells. Biol Reprod 2009; 80:657-664.

225. Dorniak P, Bazer FW, Wu G, Spencer TE. Conceptus-derived prostaglandins regulate endometrial function in sheep. Biol Reprod 2012; 87:9, 1 -7.

226. Lim H, Gupta RA, Ma WG, Paria BC, Moller DE, Morrow JD, DuBois RN, Trzaskos JM, Dey SK. Cyclo-oxygenase-2-derived prostacyclin mediates embryo implantation in the mouse via PPARdelta. Genes Dev 1999; 13:1561-1574.

227. Barak Y, Nelson MC, Ong ES, Jones YZ, Ruiz-Lozano P, Chien KR, Koder A, Evans RM. PPAR gamma is required for placental, cardiac, and adipose tissue development. Mol Cell 1999; 4:585-595.

228. Kraeling RR, Rampacek GB, Fiorello NA. Inhibition of pregnancy with indomethacin in mature gilts and prepuberal gilts induced to ovulate. Biol Reprod 1985; 32:105-110.

229. Lim H, Paria BC, Das SK, Dinchuk JE, Langenbach R, Trzaskos JM, Dey SK. Multiple female reproductive failures in cyclooxygenase 2-deficient mice. Cell 1997; 91:197-208.

230. Wang H, Ma WG, Tejada L, Zhang H, Morrow JD, Das SK, Dey SK. Rescue of female infertility from the loss of cyclooxygenase-2 by compensatory up- 
regulation of cyclooxygenase- 1 is a function of genetic makeup. $\mathrm{J}$ Biol Chem 2004; 279:10649-10658.

231. Dinchuk JE, Car BD, Focht RJ, Johnston JJ, Jaffee BD, Covington MB, Contel NR, Eng VM, Collins RJ, Czerniak PM, et al. Renal abnormalities and an altered inflammatory response in mice lacking cyclooxygenase II. Nature 1995; 378:406-409.

232. Pfeiffer CA, Meyer AE, Brooks KE, Chen PR, Milano-Foster J, Spate LD, Benne JA, Cecil RF, Samuel MS, Ciernia LA, Spinka CM, Smith MF, et al. Ablation of conceptus PTGS2 expression does not alter early conceptus development and establishment of pregnancy in the pig. Biol Reprod 2020; 102:475-488.

233. Feingold KR, Grunfeld $C$. Introduction to Lipids and Lipoproteins. In: Feingold KR, Anawalt B, Boyce A, Chrousos G, de Herder WW, Dungan K, Grossman A, Hershman JM, Hofland HJ, Kaltsas G, Koch C, Kopp P, et al. (eds.), Endotext. South Dartmouth (MA); 2000.

234. Hegele RA. Plasma lipoproteins: genetic influences and clinical implications. Nat Rev Genet 2009; 10:109-121.

235. Zilversmit DB. Atherogenic nature of triglycerides, postprandial lipidemia, and triglyceride-rich remnant lipoproteins. Clin Chem 1995; 41:153-158.

236. van Leeuwen EM, Emri E, Merle BMJ, Colijn JM, Kersten E, CougnardGregoire A, Dammeier S, Meester-Smoor M, Pool FM, de Jong EK, Delcourt C, Rodrigez-Bocanegra E, et al. A new perspective on lipid research in age-related macular degeneration. Prog Retin Eye Res 2018; 67:56-86.

237. Fraser R. Size and lipid composition of chylomicrons of different Svedberg units of flotation. J Lipid Res 1970; 11:60-65.

238. Zilversmit DB. The composition and structure of lymph chylomicrons in dog, rat, and man. J Clin Invest 1965; 44:1610-1622.

239. Altmann SW, Davis HR, Jr., Zhu LJ, Yao X, Hoos LM, Tetzloff G, lyer SP, Maguire M, Golovko A, Zeng M, Wang L, Murgolo N, et al. Niemann-Pick C1 Like 1 protein is critical for intestinal cholesterol absorption. Science 2004; 303:1201-1204.

240. Lee MH, Lu K, Hazard S, Yu H, Shulenin S, Hidaka H, Kojima H, Allikmets R, Sakuma N, Pegoraro R, Srivastava AK, Salen G, et al. Identification of a gene, ABCG5, important in the regulation of dietary cholesterol absorption. Nat Genet 2001; 27:79-83.

241. Drover VA, Ajmal M, Nassir F, Davidson NO, Nauli AM, Sahoo D, Tso P, Abumrad NA. CD36 deficiency impairs intestinal lipid secretion and clearance of chylomicrons from the blood. J Clin Invest 2005; 115:12901297.

242. Mattson FH, Volpenhein RA. Hydrolysis of primary and secondary esters of glycerol by pancreatic juice. J Lipid Res 1968; 9:79-84.

243. Chen SH, Habib G, Yang CY, Gu ZW, Lee BR, Weng SA, Silberman SR, Cai SJ, Deslypere JP, Rosseneu M, et al. Apolipoprotein B-48 is the product of a messenger RNA with an organ-specific in-frame stop codon. Science 1987; 238:363-366. 
244. Fisher EA, Ginsberg HN. Complexity in the secretory pathway: the assembly and secretion of apolipoprotein B-containing lipoproteins. J Biol Chem 2002; 277:17377-17380.

245. Mansbach CM, 2nd, Gorelick F. Development and physiological regulation of intestinal lipid absorption. II. Dietary lipid absorption, complex lipid synthesis, and the intracellular packaging and secretion of chylomicrons. Am J Physiol Gastrointest Liver Physiol 2007; 293:G645-650.

246. Wetterau JR, Aggerbeck LP, Bouma ME, Eisenberg C, Munck A, Hermier M, Schmitz J, Gay G, Rader DJ, Gregg RE. Absence of microsomal triglyceride transfer protein in individuals with abetalipoproteinemia. Science 1992; 258:999-1001.

247. Siddiqi SA, Gorelick FS, Mahan JT, Mansbach CM, 2nd. COPII proteins are required for Golgi fusion but not for endoplasmic reticulum budding of the pre-chylomicron transport vesicle. J Cell Sci 2003; 116:415-427.

248. Segrest JP, Jones MK, De Loof H, Brouillette CG, Venkatachalapathi YV, Anantharamaiah GM. The amphipathic helix in the exchangeable apolipoproteins: a review of secondary structure and function. J Lipid Res 1992; 33:141-166.

249. Beigneux AP, Davies BS, Gin P, Weinstein MM, Farber E, Qiao X, Peale F, Bunting S, Walzem RL, Wong JS, Blaner WS, Ding ZM, et al. Glycosylphosphatidylinositol-anchored high-density lipoprotein-binding protein 1 plays a critical role in the lipolytic processing of chylomicrons. Cell Metab 2007; 5:279-291.

250. LaRosa JC, Levy RI, Herbert P, Lux SE, Fredrickson DS. A specific apoprotein activator for lipoprotein lipase. Biochem Biophys Res Commun 1970; 41:57-62.

251. Futamura M, Dhanasekaran $P$, Handa T, Phillips MC, Lund-Katz S, Saito $H$. Two-step mechanism of binding of apolipoprotein $E$ to heparin: implications for the kinetics of apolipoprotein E-heparan sulfate proteoglycan complex formation on cell surfaces. J Biol Chem 2005; 280:5414-5422.

252. Bijvoet S, Gagne SE, Moorjani S, Gagne C, Henderson HE, Fruchart JC, Dallongeville J, Alaupovic P, Prins M, Kastelein JJ, Hayden MR. Alterations in plasma lipoproteins and apolipoproteins before the age of 40 in heterozygotes for lipoprotein lipase deficiency. J Lipid Res 1996; 37:640650.

253. Tiwari S, Siddiqi SA. Intracellular trafficking and secretion of VLDL. Arterioscler Thromb Vasc Biol 2012; 32:1079-1086.

254. Fisher EA, Pan M, Chen X, Wu X, Wang H, Jamil H, Sparks JD, Williams KJ. The triple threat to nascent apolipoprotein B. Evidence for multiple, distinct degradative pathways. J Biol Chem 2001; 276:27855-27863.

255. Fisher EA, Zhou M, Mitchell DM, Wu X, Omura S, Wang H, Goldberg AL, Ginsberg HN. The degradation of apolipoprotein B100 is mediated by the ubiquitin-proteasome pathway and involves heat shock protein 70 . J Biol Chem 1997; 272:20427-20434. 
256. Sacks FM. The crucial roles of apolipoproteins E and C-III in apoB lipoprotein metabolism in normolipidemia and hypertriglyceridemia. Curr Opin Lipidol 2015; 26:56-63.

257. Mcllhargey TL, Yang Y, Wong H, Hill JS. Identification of a lipoprotein lipase cofactor-binding site by chemical cross-linking and transfer of apolipoprotein C-II-responsive lipolysis from lipoprotein lipase to hepatic lipase. J Biol Chem 2003; 278:23027-23035.

258. Shimizugawa T, Ono M, Shimamura M, Yoshida K, Ando Y, Koishi R, Ueda $\mathrm{K}$, Inaba T, Minekura H, Kohama T, Furukawa H. ANGPTL3 decreases very low density lipoprotein triglyceride clearance by inhibition of lipoprotein lipase. J Biol Chem 2002; 277:33742-33748.

259. Skipski VP, Barclay M, Barclay RK, Fetzer VA, Good JJ, Archibald FM. Lipid composition of human serum lipoproteins. Biochem J 1967; 104:340-352.

260. Ference BA, Ginsberg HN, Graham I, Ray KK, Packard CJ, Bruckert E, Hegele RA, Krauss RM, Raal FJ, Schunkert H, Watts GF, Boren J, et al. Low-density lipoproteins cause atherosclerotic cardiovascular disease. 1. Evidence from genetic, epidemiologic, and clinical studies. A consensus statement from the European Atherosclerosis Society Consensus Panel. Eur Heart J 2017; 38:2459-2472.

261. Beisiegel U, Weber W, Bengtsson-Olivecrona G. Lipoprotein lipase enhances the binding of chylomicrons to low density lipoprotein receptorrelated protein. Proc Natl Acad Sci U S A 1991; 88:8342-8346.

262. Brown MS, Goldstein JL. A proteolytic pathway that controls the cholesterol content of membranes, cells, and blood. Proc Natl Acad Sci U S A 1999; 96:11041-11048.

263. Brown MS, Goldstein JL. Cholesterol feedback: from Schoenheimer's bottle to Scap's MELADL. J Lipid Res 2009; 50 Suppl:S15-27.

264. Vaisman BL, Lambert G, Amar M, Joyce C, Ito T, Shamburek RD, Cain WJ, Fruchart-Najib J, Neufeld ED, Remaley AT, Brewer HB, Jr., SantamarinaFojo S. ABCA1 overexpression leads to hyperalphalipoproteinemia and increased biliary cholesterol excretion in transgenic mice. J Clin Invest 2001; 108:303-309.

265. Smith JD, Le Goff W, Settle M, Brubaker G, Waelde C, Horwitz A, Oda MN. ABCA1 mediates concurrent cholesterol and phospholipid efflux to apolipoprotein A-I. J Lipid Res 2004; 45:635-644.

266. Wang S, Smith JD. ABCA1 and nascent HDL biogenesis. Biofactors 2014; 40:547-554.

267. Subbaiah PV, Albers JJ, Chen CH, Bagdade JD. Low density lipoproteinactivated lysolecithin acylation by human plasma lecithin-cholesterol acyltransferase. Identity of lysolecithin acyltransferase and lecithincholesterol acyltransferase. J Biol Chem 1980; 255:9275-9280.

268. Lewis GF, Rader DJ. New insights into the regulation of HDL metabolism and reverse cholesterol transport. Circ Res 2005; 96:1221-1232.

269. Gordon SM, Hofmann S, Askew DS, Davidson WS. High density lipoprotein: it's not just about lipid transport anymore. Trends Endocrinol Metab 2011; 22:9-15. 
270. Yuhanna IS, Zhu Y, Cox BE, Hahner LD, Osborne-Lawrence S, Lu P, Marcel YL, Anderson RG, Mendelsohn ME, Hobbs HH, Shaul PW. Highdensity lipoprotein binding to scavenger receptor-BI activates endothelial nitric oxide synthase. Nat Med 2001; 7:853-857.

271. Acton S, Rigotti A, Landschulz KT, Xu S, Hobbs HH, Krieger M. Identification of scavenger receptor SR-BI as a high density lipoprotein receptor. Science 1996; 271:518-520.

272. Thuahnai ST, Lund-Katz S, Williams DL, Phillips MC. Scavenger receptor class B, type I-mediated uptake of various lipids into cells. Influence of the nature of the donor particle interaction with the receptor. J Biol Chem 2001; 276:43801-43808.

273. Hammad SM, Barth JL, Knaak C, Argraves WS. Megalin acts in concert with cubilin to mediate endocytosis of high density lipoproteins. J Biol Chem 2000; 275:12003-12008.

274. Lusis AJ, Pajukanta P. A treasure trove for lipoprotein biology. Nat Genet 2008; 40:129-130.

275. Leat WM, Kubasek FO, Buttress N. Plasma lipoproteins of lambs and sheep. Q J Exp Physiol Cogn Med Sci 1976; 61:193-202.

276. Kozat S, Denizhan V. Glucose, lipid, and lipoprotein levels in sheep naturally infected with Fasciola hepatica. J Parasitol 2010; 96:657-659.

277. Langlais J, Kan FW, Granger L, Raymond L, Bleau G, Roberts KD. Identification of sterol acceptors that stimulate cholesterol efflux from human spermatozoa during in vitro capacitation. Gamete Res 1988; 20:185-201.

278. Travis AJ, Kopf GS. The role of cholesterol efflux in regulating the fertilization potential of mammalian spermatozoa. J Clin Invest 2002; 110:731-736.

279. Azhar S, Tsai L, Medicherla S, Chandrasekher Y, Giudice L, Reaven E. Human granulosa cells use high density lipoprotein cholesterol for steroidogenesis. J Clin Endocrinol Metab 1998; 83:983-991.

280. Lai WA, Yeh YT, Lee MT, Wu LS, Ke FC, Hwang JJ. Ovarian granulosa cells utilize scavenger receptor SR-BI to evade cellular cholesterol homeostatic control for steroid synthesis. J Lipid Res 2013; 54:365-378.

281. Brosens JJ, Hodgetts A, Feroze-Zaidi F, Sherwin JR, Fusi L, Salker MS, Higham J, Rose GL, Kajihara T, Young SL, Lessey BA, Henriet P, et al. Proteomic analysis of endometrium from fertile and infertile patients suggests a role for apolipoprotein A-I in embryo implantation failure and endometriosis. Mol Hum Reprod 2010; 16:273-285.

282. Sherwin JR, Sharkey AM, Cameo P, Mavrogianis PM, Catalano RD, Edassery S, Fazleabas AT. Identification of novel genes regulated by chorionic gonadotropin in baboon endometrium during the window of implantation. Endocrinology 2007; 148:618-626.

283. Kao LC, Tulac S, Lobo S, Imani B, Yang JP, Germeyer A, Osteen K, Taylor RN, Lessey BA, Giudice LC. Global gene profiling in human endometrium during the window of implantation. Endocrinology 2002; 143:2119-2138. 
284. Argraves WS, Morales CR. Immunolocalization of cubilin, megalin, apolipoprotein $\mathrm{J}$, and apolipoprotein A-I in the uterus and oviduct. Mol Reprod Dev 2004; 69:419-427.

285. Gou J, Jia J, Zhao X, Yi T, Li Z. Identification of stathmin 1 during periimplantation period in mouse endometrium by a proteomics-based analysis. Biochem Biophys Res Commun 2015; 461:211-216.

286. Jia J, Gou J, Zhao X, Yi T, Li Z. Apolipoprotein A1 and heterogeneous nuclear ribonucleoprotein $\mathrm{E} 1$ implicated in the regulation of embryo implantation by inhibiting lipid peroxidation. Reprod Biomed Online 2016; 33:635-645.

287. Killeen AP, Diskin MG, Morris DG, Kenny DA, Waters SM. Endometrial gene expression in high- and low-fertility heifers in the late luteal phase of the estrous cycle and a comparison with midluteal gene expression. Physiol Genomics 2016; 48:306-319.

288. Forde N, Bazer FW, Spencer TE, Lonergan P. 'Conceptualizing' the Endometrium: Identification of Conceptus-Derived Proteins During Early Pregnancy in Cattle. Biol Reprod 2015; 92:156.

289. Mains LM, Christenson L, Yang B, Sparks AE, Mathur S, Van Voorhis BJ. Identification of apolipoprotein A1 in the human embryonic secretome. Fertil Steril 2011; 96:422-427 e422.

290. Huang LS, Voyiaziakis E, Markenson DF, Sokol KA, Hayek T, Breslow JL. apo $B$ gene knockout in mice results in embryonic lethality in homozygotes and neural tube defects, male infertility, and reduced HDL cholesterol ester and apo A-I transport rates in heterozygotes. J Clin Invest 1995; 96:21522161.

291. Farese RV, Jr., Ruland SL, Flynn LM, Stokowski RP, Young SG. Knockout of the mouse apolipoprotein $B$ gene results in embryonic lethality in homozygotes and protection against diet-induced hypercholesterolemia in heterozygotes. Proc Natl Acad Sci U S A 1995; 92:1774-1778.

292. Farese RV, Jr., Cases S, Ruland SL, Kayden HJ, Wong JS, Young SG, Hamilton RL. A novel function for apolipoprotein B: lipoprotein synthesis in the yolk sac is critical for maternal-fetal lipid transport in mice. J Lipid Res 1996; 37:347-360.

293. Christiansen-Weber TA, Voland JR, Wu Y, Ngo K, Roland BL, Nguyen S, Peterson PA, Fung-Leung WP. Functional loss of ABCA1 in mice causes severe placental malformation, aberrant lipid distribution, and kidney glomerulonephritis as well as high-density lipoprotein cholesterol deficiency. Am J Pathol 2000; 157:1017-1029.

294. Miettinen HE, Rayburn H, Krieger M. Abnormal lipoprotein metabolism and reversible female infertility in HDL receptor (SR-BI)-deficient mice. J Clin Invest 2001; 108:1717-1722.

295. Li H, Reddick RL, Maeda N. Lack of apoA-I is not associated with increased susceptibility to atherosclerosis in mice. Arterioscler Thromb 1993; 13:1814-1821.

296. Spencer TE, Johnson GA, Bazer FW, Burghardt RC. Implantation mechanisms: insights from the sheep. Reproduction 2004; 128:657-668. 
297. Brooks K, Spencer TE. Biological roles of interferon tau (IFNT) and type I IFN receptors in elongation of the ovine conceptus. Biol Reprod 2015; 92:47.

298. Roberts RM, Xie S, Mathialagan N. Maternal recognition of pregnancy. Biol Reprod 1996; 54:294-302.

299. Spencer TE, Forde N, Lonergan $P$. The role of progesterone and conceptus-derived factors in uterine biology during early pregnancy in ruminants. J Dairy Sci 2016; 99:5941-5950.

300. Spencer TE, Hansen TR. Implantation and Establishment of Pregnancy in Ruminants. Adv Anat Embryol Cell Biol 2015; 216:105-135.

301. Spencer TE, Johnson GA, Bazer FW, Burghardt RC. Fetal-maternal interactions during the establishment of pregnancy in ruminants. Soc Reprod Fertil Suppl 2007; 64:379-396.

302. Spencer TE, Sandra O, Wolf E. Genes involved in conceptus-endometrial interactions in ruminants: insights from reductionism and thoughts on holistic approaches. Reproduction 2008; 135:165-179.

303. Forde N, Carter F, Fair T, Crowe MA, Evans AC, Spencer TE, Bazer FW, McBride R, Boland MP, O'Gaora P, Lonergan P, Roche JF. Progesteroneregulated changes in endometrial gene expression contribute to advanced conceptus development in cattle. Biol Reprod 2009; 81:784-794.

304. Kelleher AM, DeMayo FJ, Spencer TE. Uterine Glands: Developmental Biology and Functional Roles in Pregnancy. Endocr Rev 2019; 40:14241445.

305. Allison Gray C, Bartol FF, Taylor KM, Wiley AA, Ramsey WS, Ott TL, Bazer FW, Spencer TE. Ovine uterine gland knock-out model: effects of gland ablation on the estrous cycle. Biol Reprod 2000; 62:448-456.

306. S ELA, Mager I, Breakefield XO, Wood MJ. Extracellular vesicles: biology and emerging therapeutic opportunities. Nat Rev Drug Discov 2013; 12:347-357.

307. van Niel G, D'Angelo G, Raposo G. Shedding light on the cell biology of extracellular vesicles. Nat Rev Mol Cell Biol 2018; 19:213-228.

308. Racicot K, Schmitt A, Ott T. The myxovirus-resistance protein, MX1, is a component of exosomes secreted by uterine epithelial cells. Am J Reprod Immunol 2012; 67:498-505.

309. Spencer TE, Johnson GA, Burghardt RC, Bazer FW. Progesterone and placental hormone actions on the uterus: insights from domestic animals. Biol Reprod 2004; 71:2-10.

310. Van Heeke G, Ott TL, Strauss A, Ammaturo D, Bazer FW. High yield expression and secretion of the ovine pregnancy recognition hormone interferon-tau by Pichia pastoris. J Interferon Cytokine Res 1996; 16:119126.

311. Ashworth CJ, Bazer FW. Changes in ovine conceptus and endometrial function following asynchronous embryo transfer or administration of progesterone. Biol Reprod 1989; 40:425-433.

312. Bazer FW, Spencer TE. Methods for studying interferon tau stimulated genes. Methods Mol Med 2006; 122:367-380. 
313. Lobb RJ, Becker M, Wen SW, Wong CS, Wiegmans AP, Leimgruber A, Moller A. Optimized exosome isolation protocol for cell culture supernatant and human plasma. J Extracell Vesicles 2015; 4:27031.

314. Nordin JZ, Lee Y, Vader P, Mager I, Johansson HJ, Heusermann W, Wiklander OP, Hallbrink M, Seow Y, Bultema JJ, Gilthorpe J, Davies T, et al. Ultrafiltration with size-exclusion liquid chromatography for high yield isolation of extracellular vesicles preserving intact biophysical and functional properties. Nanomedicine 2015; 11:879-883.

315. Harp D, Driss A, Mehrabi S, Chowdhury I, Xu W, Liu D, Garcia-Barrio M, Taylor RN, Gold B, Jefferson S, Sidell N, Thompson W. Exosomes derived from endometriotic stromal cells have enhanced angiogenic effects in vitro. Cell Tissue Res 2016; 365:187-196.

316. Bligh EG, Dyer WJ. A rapid method of total lipid extraction and purification. Can J Biochem Physiol 1959; 37:911-917.

317. de Lima CB, Ferreira CR, Milazzotto MP, Sobreira TJP, Vireque AA, Cooks RG. Comprehensive lipid profiling of early stage oocytes and embryos by MRM profiling. J Mass Spectrom 2018; 53:1247-1252.

318. Farmer JL, Burghardt RC, Jousan FD, Hansen PJ, Bazer FW, Spencer TE. Galectin 15 (LGALS15) functions in trophectoderm migration and attachment. FASEB J 2008; 22:548-560.

319. Dunlap KA, Palmarini M, Varela M, Burghardt RC, Hayashi K, Farmer JL, Spencer TE. Endogenous retroviruses regulate periimplantation placental growth and differentiation. Proc Natl Acad Sci U S A 2006; 103:1439014395.

320. Farmer JL, Burghardt RC, Jousan FD, Hansen PJ, Bazer FW, Spencer TE. Galectin 15 (LGALS15) functions in trophectoderm migration and attachment. FASEB journal : official publication of the Federation of American Societies for Experimental Biology 2008; 22:548-560.

321. Hayashi K, Burghardt RC, Bazer FW, Spencer TE. WNTs in the ovine uterus: potential regulation of periimplantation ovine conceptus development. Endocrinology 2007; 148:3496-3506.

322. Kim D, Langmead B, Salzberg SL. HISAT: a fast spliced aligner with low memory requirements. Nat Methods 2015; 12:357-360.

323. Aken BL, Ayling S, Barrell D, Clarke L, Curwen V, Fairley S, Fernandez Banet J, Billis K, Garcia Giron C, Hourlier T, Howe K, Kahari A, et al. The Ensembl gene annotation system. Database (Oxford) 2016; 2016:1-19.

324. Liao Y, Smyth GK, Shi W. featureCounts: an efficient general purpose program for assigning sequence reads to genomic features. Bioinformatics 2014; 30:923-930.

325. Zhou X, Lindsay H, Robinson MD. Robustly detecting differential expression in RNA sequencing data using observation weights. Nucleic Acids Res 2014; 42:e91.

326. Boyle El, Weng S, Gollub J, Jin H, Botstein D, Cherry JM, Sherlock G. GO::TermFinder--open source software for accessing Gene Ontology information and finding significantly enriched Gene Ontology terms associated with a list of genes. Bioinformatics 2004; 20:3710-3715. 
327. Supek F, Bosnjak M, Skunca N, Smuc T. REVIGO summarizes and visualizes long lists of gene ontology terms. PLoS One 2011; 6:e21800.

328. Johnson GA, Spencer TE, Burghardt RC, Joyce MM, Bazer FW. Interferontau and progesterone regulate ubiquitin cross-reactive protein expression in the ovine uterus. Biol Reprod 2000; 62:622-627.

329. Lee YXF, Johansson H, Wood MJA, El Andaloussi S. Considerations and Implications in the Purification of Extracellular Vesicles - A Cautionary Tale. Front Neurosci 2019; 13:1067.

330. Spencer TE, Stagg AG, Joyce MM, Jenster G, Wood CG, Bazer FW, Wiley $A A$, Bartol FF. Discovery and characterization of endometrial epithelial messenger ribonucleic acids using the ovine uterine gland knockout model. Endocrinology 1999; 140:4070-4080.

331. Palmarini M, Gray CA, Carpenter K, Fan H, Bazer FW, Spencer TE. Expression of endogenous betaretroviruses in the ovine uterus: effects of neonatal age, estrous cycle, pregnancy, and progesterone. J Virol 2001; 75:11319-11327.

332. Dorniak P, Bazer FW, Spencer TE. Physiology and Endocrinology Symposium: biological role of interferon tau in endometrial function and conceptus elongation. J Anim Sci 2013; 91:1627-1638.

333. Ochieng J, Pratap S, Khatua AK, Sakwe AM. Anchorage-independent growth of breast carcinoma cells is mediated by serum exosomes. Exp Cell Res 2009; 315:1875-1888.

334. Dunlap KA, Palmarini M, Adelson DL, Spencer TE. Sheep endogenous betaretroviruses (enJSRVs) and the hyaluronidase 2 (HYAL2) receptor in the ovine uterus and conceptus. Biol Reprod 2005; 73:271-279.

335. Moore SG, Ericsson AC, Poock SE, Melendez P, Lucy MC. Hot topic: 16 S rRNA gene sequencing reveals the microbiome of the virgin and pregnant bovine uterus. J Dairy Sci 2017; 100:4953-4960.

336. Santos TM, Gilbert RO, Bicalho RC. Metagenomic analysis of the uterine bacterial microbiota in healthy and metritic postpartum dairy cows. J Dairy Sci 2011; 94:291-302.

337. Skotland T, Sandvig K, Llorente A. Lipids in exosomes: Current knowledge and the way forward. Prog Lipid Res 2017; 66:30-41.

338. Sodar BW, Kittel A, Paloczi K, Vukman KV, Osteikoetxea X, Szabo-Taylor K, Nemeth A, Sperlagh B, Baranyai T, Giricz Z, Wiener Z, Turiak L, et al. Low-density lipoprotein mimics blood plasma-derived exosomes and microvesicles during isolation and detection. Sci Rep 2016; 6:24316.

339. Wu M, Chen C, Wang Z, Bachman H, Ouyang Y, Huang PH, Sadovsky Y, Huang TJ. Separating extracellular vesicles and lipoproteins via acoustofluidics. Lab Chip 2019; 19:1174-1182.

340. Osteikoetxea X, Sodar B, Nemeth A, Szabo-Taylor K, Paloczi K, Vukman KV, Tamasi V, Balogh A, Kittel A, Pallinger E, Buzas El. Differential detergent sensitivity of extracellular vesicle subpopulations. Org Biomol Chem 2015; 13:9775-9782.

341. Hessvik NP, Llorente A. Current knowledge on exosome biogenesis and release. Cell Mol Life Sci 2018; 75:193-208. 
342. Dean JM, Lodhi IJ. Structural and functional roles of ether lipids. Protein Cell 2018; 9:196-206.

343. Simbari F, McCaskill J, Coakley G, Millar M, Maizels RM, Fabrias G, Casas $\mathrm{J}$, Buck AH. Plasmalogen enrichment in exosomes secreted by a nematode parasite versus those derived from its mouse host: implications for exosome stability and biology. J Extracell Vesicles 2016; 5:30741.

344. Perry DK, Hannun YA. The role of ceramide in cell signaling. Biochim Biophys Acta 1998; 1436:233-243.

345. Ruvolo PP. Ceramide regulates cellular homeostasis via diverse stress signaling pathways. Leukemia 2001; 15:1153-1160.

346. Moraes JGN, Behura SK, Geary TW, Spencer TE. Analysis of the Uterine Lumen in Fertility-Classified Heifers: I. Glucose, Prostaglandins and Lipids. Biol Reprod 2019.

347. Wang J, Guillomot M, Hue I. Cellular organization of the trophoblastic epithelium in elongating conceptuses of ruminants. C R Biol 2009; 332:986997.

348. Randi F, Fernandez-Fuertes B, McDonald M, Forde N, Kelly AK, Bastos Amorin H, Muniz de Lima E, Morotti F, Marcondes Seneda M, Lonergan P. Asynchronous embryo transfer as a tool to understand embryo-uterine interaction in cattle: is a large conceptus a good thing? Reprod Fertil Dev 2016; 28:1999-2006.

349. Sanchez JM, Mathew DJ, Behura SK, Passaro C, Charpigny G, Butler ST, Spencer TE, Lonergan P. Bovine endometrium responds differentially to age-matched short and long conceptuses. Biol Reprod 2019; 101:26-39.

350. Francis GL. Albumin and mammalian cell culture: implications for biotechnology applications. Cytotechnology 2010; 62:1-16.

351. Bourbon NA, Yun J, Kester M. Ceramide directly activates protein kinase C zeta to regulate a stress-activated protein kinase signaling complex. J Biol Chem 2000; 275:35617-35623.

352. Kim DS, Kim SY, Moon SJ, Chung JH, Kim KH, Cho KH, Park KC. Ceramide inhibits cell proliferation through Akt/PKB inactivation and decreases melanin synthesis in Mel-Ab cells. Pigment Cell Res 2001; 14:110-115.

353. Lin IL, Chou HL, Lee JC, Chen FW, Fong Y, Chang WC, Huang HW, Wu CY, Chang WT, Wang HD, Chiu CC. The antiproliferative effect of C2ceramide on lung cancer cells through apoptosis by inhibiting Akt and NFkappaB. Cancer Cell Int 2014; 14:1.

354. Twiss JL, Fainzilber M. Ribosomes in axons--scrounging from the neighbors? Trends Cell Biol 2009; 19:236-243.

355. Tanguy E, Carmon O, Wang Q, Jeandel L, Chasserot-Golaz S, MonteroHadjadje M, Vitale N. Lipids implicated in the journey of a secretory granule: from biogenesis to fusion. J Neurochem 2016; 137:904-912.

356. Bazer FW, Wu G, Spencer TE, Johnson GA, Burghardt RC, Bayless K. Novel pathways for implantation and establishment and maintenance of pregnancy in mammals. Mol Hum Reprod 2010; 16:135-152.

357. Spencer TE, Johnson GA, Bazer FW, Burghardt RC, Palmarini M. Pregnancy recognition and conceptus implantation in domestic ruminants: 
roles of progesterone, interferons and endogenous retroviruses. Reprod Fertil Dev 2007; 19:65-78.

358. Bindon BM. Systematic study of preimplantation stages of pregnancy in the sheep. Aust J Biol Sci 1971; 24:131-147.

359. O'Neil EV, Brooks K, Burns GW, Ortega MS, Denicol AC, Aguiar LH, Pedroza GH, Benne J, Spencer TE. Prostaglandin-endoperoxide synthase 2 is not required for preimplantation ovine conceptus development in sheep. Mol Reprod Dev 2020; 87:142-151.

360. Heyman Y, Camous S, Fevre J, Meziou W, Martal J. Maintenance of the corpus luteum after uterine transfer of trophoblastic vesicles to cyclic cows and ewes. J Reprod Fertil 1984; 70:533-540.

361. Bazer FW, Wang X, Johnson GA, Wu G. Select nutrients and their effects on conceptus development in mammals. Anim Nutr 2015; 1:85-95.

362. Gyorgy B, Szabo TG, Pasztoi M, Pal Z, Misjak P, Aradi B, Laszlo V, Pallinger E, Pap E, Kittel A, Nagy G, Falus A, et al. Membrane vesicles, current state-of-the-art: emerging role of extracellular vesicles. Cell Mol Life Sci 2011; 68:2667-2688.

363. Raposo G, Stoorvogel W. Extracellular vesicles: exosomes, microvesicles, and friends. J Cell Biol 2013; 200:373-383.

364. O'Neil EV, Burns GW, Ferreira CR, Spencer TE. Characterization and regulation of extracellular vesicles in the lumen of the ovine uterus. Biol Reprod 2020; 102:1020-1032.

365. Akbarzadeh A, Rezaei-Sadabady R, Davaran S, Joo SW, Zarghami N, Hanifehpour Y, Samiei M, Kouhi M, Nejati-Koshki K. Liposome: classification, preparation, and applications. Nanoscale Res Lett 2013; 8:102.

366. Antimisiaris SG, Mourtas S, Marazioti A. Exosomes and Exosome-Inspired Vesicles for Targeted Drug Delivery. Pharmaceutics 2018; 10.

367. Martini C, Pallottini V. Cholesterol: from feeding to gene regulation. Genes Nutr 2007; 2:181-193.

368. Nakamura MT, Cheon Y, Li Y, Nara TY. Mechanisms of regulation of gene expression by fatty acids. Lipids 2004; 39:1077-1083.

369. Walker AK, Jacobs RL, Watts JL, Rottiers V, Jiang K, Finnegan DM, Shioda $T$, Hansen M, Yang F, Niebergall LJ, Vance DE, Tzoneva M, et al. A conserved SREBP-1/phosphatidylcholine feedback circuit regulates lipogenesis in metazoans. Cell 2011; 147:840-852.

370. McAndrews KM, Kalluri R. Mechanisms associated with biogenesis of exosomes in cancer. Mol Cancer 2019; 18:52.

371. Salomon C, Ryan J, Sobrevia L, Kobayashi M, Ashman K, Mitchell M, Rice GE. Exosomal signaling during hypoxia mediates microvascular endothelial cell migration and vasculogenesis. PLoS One 2013; 8:e68451.

372. Tabak S, Schreiber-Avissar S, Beit-Yannai E. Extracellular vesicles have variable dose-dependent effects on cultured draining cells in the eye. $\mathrm{J}$ Cell Mol Med 2018; 22:1992-2000. 
373. Xu J, Wang Y, Hsu CY, Gao Y, Meyers CA, Chang L, Zhang L, Broderick K, Ding C, Peault B, Witwer K, James AW. Human perivascular stem cellderived extracellular vesicles mediate bone repair. Elife 2019; 8.

374. Prada I, Meldolesi J. Binding and Fusion of Extracellular Vesicles to the Plasma Membrane of Their Cell Targets. Int J Mol Sci 2016; 17.

375. Skotland T, Sagini K, Sandvig K, Llorente A. An emerging focus on lipids in extracellular vesicles. Adv Drug Deliv Rev 2020.

376. Tosti E, Boni R, Gallo A. lon currents in embryo development. Birth Defects Res C Embryo Today 2016; 108:6-18.

377. Schwanhausser B, Busse D, Li N, Dittmar G, Schuchhardt J, Wolf J, Chen W, Selbach M. Global quantification of mammalian gene expression control. Nature 2011; 473:337-342.

378. Chandrasekharan NV, Simmons DL. The cyclooxygenases. Genome Biol 2004; 5:241.

379. Spencer TE, Ing NH, Ott TL, Mayes JS, Becker WC, Watson GH, Mirando MA, Brazer FW. Intrauterine injection of ovine interferon-tau alters oestrogen receptor and oxytocin receptor expression in the endometrium of cyclic ewes. J Mol Endocrinol 1995; 15:203-220.

380. Ayad VJ, Matthews EL, Wathes DC, Parkinson TJ, Wild ML. Autoradiographic localization of oxytocin receptors in the endometrium during the oestrous cycle of the ewe. J Endocrinol 1991; 130:199-206.

381. McCracken JA, Carlson JC, Glew ME, Goding JR, Baird DT, Green K, Samuelsson B. Prostaglandin F 2 identified as a luteolytic hormone in sheep. Nat New Biol 1972; 238:129-134.

382. Hugentobler SA, Diskin MG, Leese HJ, Humpherson PG, Watson T, Sreenan JM, Morris DG. Amino acids in oviduct and uterine fluid and blood plasma during the estrous cycle in the bovine. Mol Reprod Dev 2007; 74:445-454.

383. Song G, Bazer FW, Spencer TE. Pregnancy and interferon tau regulate RSAD2 and IFIH1 expression in the ovine uterus. Reproduction 2007; 133:285-295.

384. Morris DG, Humpherson PG, Leese HJ, Sreenan JM. Amino acid turnover by elongating cattle blastocysts recovered on days 14-16 after insemination. Reproduction 2002; 124:667-673.

385. Matyash V, Liebisch G, Kurzchalia TV, Shevchenko A, Schwudke D. Lipid extraction by methyl-tert-butyl ether for high-throughput lipidomics. J Lipid Res 2008; 49:1137-1146.

386. Mehlem A, Hagberg CE, Muhl L, Eriksson U, Falkevall A. Imaging of neutral lipids by oil red $\mathrm{O}$ for analyzing the metabolic status in health and disease. Nat Protoc 2013; 8:1149-1154.

387. Rueden CT, Schindelin J, Hiner MC, DeZonia BE, Walter AE, Arena ET, Eliceiri KW. ImageJ2: ImageJ for the next generation of scientific image data. BMC Bioinformatics 2017; 18:529.

388. Schneider CA, Rasband WS, Eliceiri KW. NIH Image to ImageJ: 25 years of image analysis. Nat Methods 2012; 9:671-675. 
389. Simintiras CA, Sanchez JM, McDonald M, Martins T, Binelli M, Lonergan P. Biochemical characterization of progesterone-induced alterations in bovine uterine fluid amino acid and carbohydrate composition during the conceptus elongation window. Biol Reprod 2019; 100:672-685.

390. Levental KR, Malmberg E, Symons JL, Fan YY, Chapkin RS, Ernst R, Levental I. Lipidomic and biophysical homeostasis of mammalian membranes counteracts dietary lipid perturbations to maintain cellular fitness. Nat Commun 2020; 11:1339.

391. Tai K, Cockburn K, Greco V. Flexibility sustains epithelial tissue homeostasis. Curr Opin Cell Biol 2019; 60:84-91.

392. Bartz R, Li WH, Venables B, Zehmer JK, Roth MR, Welti R, Anderson RG, Liu $\mathrm{P}$, Chapman KD. Lipidomics reveals that adiposomes store ether lipids and mediate phospholipid traffic. J Lipid Res 2007; 48:837-847.

393. Olzmann JA, Carvalho P. Dynamics and functions of lipid droplets. Nat Rev Mol Cell Biol 2019; 20:137-155.

394. Murphy RC, Hankin JA, Barkley RM. Imaging of lipid species by MALDI mass spectrometry. J Lipid Res 2009; 50 Suppl:S317-322.

395. Uzbekova S, Elis S, Teixeira-Gomes AP, Desmarchais A, Maillard V, Labas V. MALDI Mass Spectrometry Imaging of Lipids and Gene Expression Reveals Differences in Fatty Acid Metabolism between Follicular Compartments in Porcine Ovaries. Biology (Basel) 2015; 4:216-236.

396. van der Veen JN, Kennelly JP, Wan S, Vance JE, Vance DE, Jacobs RL. The critical role of phosphatidylcholine and phosphatidylethanolamine metabolism in health and disease. Biochim Biophys Acta Biomembr 2017; 1859:1558-1572.

397. Makieva S, Giacomini E, Ottolina J, Sanchez AM, Papaleo E, Vigano P. Inside the Endometrial Cell Signaling Subway: Mind the Gap(s). Int J Mol Sci 2018; 19.

398. Prestwich GD. Phosphoinositide signaling; from affinity probes to pharmaceutical targets. Chem Biol 2004; 11:619-637.

399. Alb JG, Jr., Phillips SE, Rostand K, Cui X, Pinxteren J, Cotlin L, Manning T, Guo S, York JD, Sontheimer H, Collawn JF, Bankaitis VA. Genetic ablation of phosphatidylinositol transfer protein function in murine embryonic stem cells. Mol Biol Cell 2002; 13:739-754.

400. Monks J, Ladinsky MS, McManaman JL. Organellar Contacts of Milk Lipid Droplets. Contact (Thousand Oaks) 2020; 3.

401. McManaman JL. Milk lipid secretion: recent biomolecular aspects. Biomol Concepts 2012; 3:581-591.

402. Meier S, Trewhella MA, Fairclough RJ, Jenkin G. Changes in uterine endometrial phospholipids and fatty acids throughout the oestrous cycle and early pregnancy in the ewe. Prostaglandins Leukot Essent Fatty Acids 1997; 57:341-349.

403. Sztalryd C, Brasaemle DL. The perilipin family of lipid droplet proteins: Gatekeepers of intracellular lipolysis. Biochim Biophys Acta Mol Cell Biol Lipids 2017; 1862:1221-1232. 
404. Helander HF, Olivecrona T. Lipolysis and lipid absorption in the stomach of the suckling rat. Gastroenterology 1970; 59:22-35.

405. Hussain MM. Intestinal lipid absorption and lipoprotein formation. Curr Opin Lipidol 2014; 25:200-206.

406. Crutchfield FL, Kulangara AC. Passage of bovine serum albumin from the mother to rabbit blastocysts. I. Passage from the circulation to uterine lumen. J Embryol Exp Morphol 1973; 30:459-469.

407. McRae AC. The blood-uterine lumen barrier and exchange between extracellular fluids. J Reprod Fertil 1988; 82:857-873.

408. Bieberich E. Ceramide signaling in cancer and stem cells. Future Lipidol 2008; 3:273-300.

409. Ferguson EM, Leese HJ. A potential role for triglyceride as an energy source during bovine oocyte maturation and early embryo development. Mol Reprod Dev 2006; 73:1195-1201.

410. Schooneman MG, Vaz FM, Houten SM, Soeters MR. Acylcarnitines: reflecting or inflicting insulin resistance? Diabetes 2013; 62:1-8.

411. Xiao D, Zeng L, Yao K, Kong X, Wu G, Yin Y. The glutamine-alphaketoglutarate (AKG) metabolism and its nutritional implications. Amino Acids 2016; 48:2067-2080.

412. Yao K, Yin Y, Li X, Xi P, Wang J, Lei J, Hou Y, Wu G. Alpha-ketoglutarate inhibits glutamine degradation and enhances protein synthesis in intestinal porcine epithelial cells. Amino Acids 2012; 42:2491-2500.

413. Hostetler CE, Kincaid RL, Mirando MA. The role of essential trace elements in embryonic and fetal development in livestock. Vet J 2003; 166:125-139.

414. Cluntun AA, Lukey MJ, Cerione RA, Locasale JW. Glutamine Metabolism in Cancer: Understanding the Heterogeneity. Trends Cancer 2017; 3:169180.

415. Kim J, Burghardt RC, Wu G, Johnson GA, Spencer TE, Bazer FW. Select nutrients in the ovine uterine lumen. IX. Differential effects of arginine, leucine, glutamine, and glucose on interferon tau, ornithine decarboxylase, and nitric oxide synthase in the ovine conceptus. Biol Reprod 2011; 84:1139-1147.

416. Wen HY, Abbasi S, Kellems RE, Xia Y. mTOR: a placental growth signaling sensor. Placenta 2005; 26 Suppl A:S63-69.

417. Chong J, Wishart DS, Xia J. Using MetaboAnalyst 4.0 for Comprehensive and Integrative Metabolomics Data Analysis. Curr Protoc Bioinformatics 2019; 68:e86.

418. Pang Z, Chong J, Li S, Xia J. MetaboAnalystR 3.0: Toward an Optimized Workflow for Global Metabolomics. Metabolites 2020; 10.

419. Bazer FW, Song G, Kim J, Erikson DW, Johnson GA, Burghardt RC, Gao $\mathrm{H}$, Carey Satterfield M, Spencer TE, Wu G. Mechanistic mammalian target of rapamycin (MTOR) cell signaling: effects of select nutrients and secreted phosphoprotein 1 on development of mammalian conceptuses. Mol Cell Endocrinol 2012; 354:22-33.

420. Shambaugh GE, 3rd. Urea biosynthesis I. The urea cycle and relationships to the citric acid cycle. Am J Clin Nutr 1977; 30:2083-2087. 
421. Gardner DK. Lactate production by the mammalian blastocyst: manipulating the microenvironment for uterine implantation and invasion? Bioessays 2015; 37:364-371.

422. Rhoads ML, Gilbert RO, Lucy MC, Butler WR. Effects of urea infusion on the uterine luminal environment of dairy cows. J Dairy Sci 2004; 87:28962901.

423. Williamson JR, Safer B, LaNoue KF, Smith CM, Walajtys E. Mitochondrialcytosolic interactions in cardiac tissue: role of the malate-aspartate cycle in the removal of glycolytic NADH from the cytosol. Symp Soc Exp Biol 1973; 27:241-281.

424. Coelho AI, Trabuco M, Silva MJ, de Almeida IT, Leandro P, Rivera I, Vicente JB. Arginine Functionally Improves Clinically Relevant Human Galactose1-Phosphate Uridylyltransferase (GALT) Variants Expressed in a Prokaryotic Model. JIMD Rep 2015; 23:1-6.

425. Nichols BL, Avery S, Sen P, Swallow DM, Hahn D, Sterchi E. The maltaseglucoamylase gene: common ancestry to sucrase-isomaltase with complementary starch digestion activities. Proc Natl Acad Sci U S A 2003; 100:1432-1437.

426. Jefferies H, Coster J, Khalil A, Bot J, McCauley RD, Hall JC. Glutathione. ANZ J Surg 2003; 73:517-522.

427. Madeo F, Eisenberg T, Pietrocola F, Kroemer G. Spermidine in health and disease. Science 2018; 359.

428. Hamczyk MR, Villa-Bellosta R. Pyrophosphate metabolism and calcification. Aging (Albany NY) 2018; 10:3652-3653.

429. Guillomot M, Flechon JE, Wintenberger-Torres S. Conceptus attachment in the ewe: an ultrastructural study. Placenta 1981; 2:169-182.

430. Marcus GJ. Prostaglandin formation by the sheep embryo and endometrium as an indication of maternal recognition of pregnancy. Biol Reprod 1981; 25:56-64.

431. Arosh JA, Banu SK, Kimmins S, Chapdelaine P, Maclaren LA, Fortier MA. Effect of interferon-tau on prostaglandin biosynthesis, transport, and signaling at the time of maternal recognition of pregnancy in cattle: evidence of polycrine actions of prostaglandin E2. Endocrinology 2004; 145:52805293.

432. Emond V, MacLaren LA, Kimmins S, Arosh JA, Fortier MA, Lambert RD. Expression of cyclooxygenase-2 and granulocyte-macrophage colonystimulating factor in the endometrial epithelium of the cow is up-regulated during early pregnancy and in response to intrauterine infusions of interferon-tau. Biol Reprod 2004; 70:54-64.

433. Simmons RM, Erikson DW, Kim J, Burghardt RC, Bazer FW, Johnson GA, Spencer TE. Insulin-like growth factor binding protein-1 in the ruminant uterus: potential endometrial marker and regulator of conceptus elongation. Endocrinology 2009; 150:4295-4305.

434. Patel O, Dumesny C, Shulkes A, Baldwin GS. C-terminal fragments of the gastrin-releasing peptide precursor stimulate cell proliferation via a novel receptor. Endocrinology 2007; 148:1330-1339. 
435. Brooks K, Burns G, Spencer TE. Biological Roles of Hydroxysteroid (11Beta) Dehydrogenase 1 (HSD11B1), HSD11B2, and Glucocorticoid Receptor (NR3C1) in Sheep Conceptus Elongation. Biol Reprod 2015; 93:38.

436. Vilarino M, Rashid ST, Suchy FP, McNabb BR, van der Meulen T, Fine EJ, Ahsan SD, Mursaliyev N, Sebastiano V, Diab SS, Huising MO, Nakauchi H, et al. CRISPR/Cas9 microinjection in oocytes disables pancreas development in sheep. Sci Rep 2017; 7:17472.

437. Tervit HR, Whittingham DG, Rowson LE. Successful culture in vitro of sheep and cattle ova. J Reprod Fertil 1972; 30:493-497.

438. Ortega MS, Moraes JGN, Patterson DJ, Smith MF, Behura SK, Poock S, Spencer TE. Influences of sire conception rate on pregnancy establishment in dairy cattle. Biol Reprod 2018; 99:1244-1254.

439. Gasteiger E, Gattiker A, Hoogland C, Ivanyi I, Appel RD, Bairoch A. ExPASy: The proteomics server for in-depth protein knowledge and analysis. Nucleic Acids Res 2003; 31:3784-3788.

440. Benkert $P$, Biasini M, Schwede T. Toward the estimation of the absolute quality of individual protein structure models. Bioinformatics 2011; 27:343350.

441. Bertoni M, Kiefer F, Biasini M, Bordoli L, Schwede T. Modeling protein quaternary structure of homo- and hetero-oligomers beyond binary interactions by homology. Sci Rep 2017; 7:10480.

442. Bienert S, Waterhouse A, de Beer TA, Tauriello G, Studer G, Bordoli L, Schwede T. The SWISS-MODEL Repository-new features and functionality. Nucleic Acids Res 2017; 45:D313-D319.

443. Guex N, Peitsch MC, Schwede T. Automated comparative protein structure modeling with SWISS-MODEL and Swiss-PdbViewer: a historical perspective. Electrophoresis 2009; 30 Suppl 1:S162-173.

444. Waterhouse A, Bertoni M, Bienert S, Studer G, Tauriello G, Gumienny R, Heer FT, de Beer TAP, Rempfer C, Bordoli L, Lepore R, Schwede T. SWISS-MODEL: homology modelling of protein structures and complexes. Nucleic Acids Res 2018; 46:W296-W303.

445. Vecchio AJ, Malkowski MG. The structural basis of endocannabinoid oxygenation by cyclooxygenase-2. J Biol Chem 2011; 286:20736-20745.

446. El-Sayed A, Hoelker M, Rings F, Salilew D, Jennen D, Tholen E, Sirard MA, Schellander K, Tesfaye D. Large-scale transcriptional analysis of bovine embryo biopsies in relation to pregnancy success after transfer to recipients. Physiol Genomics 2006; 28:84-96.

447. Song BS, Kim JS, Kim CH, Han YM, Lee DS, Lee KK, Koo DB. Prostacyclin stimulates embryonic development via regulation of the CAMP response element-binding protein-cyclo-oxygenase-2 signalling pathway in cattle. Reprod Fertil Dev 2009; 21:400-407.

448. Sayre BL, Lewis GS. Arachidonic acid metabolism during early development of ovine embryos: a possible relationship to shedding of the zona pellucida. Prostaglandins 1993; 45:557-569. 
449. Mehravar M, Shirazi A, Nazari M, Banan M. Mosaicism in CRISPR/Cas9mediated genome editing. Dev Biol 2019; 445:156-162.

450. Yen ST, Zhang M, Deng JM, Usman SJ, Smith CN, Parker-Thornburg J, Swinton PG, Martin JF, Behringer RR. Somatic mosaicism and allele complexity induced by CRISPR/Cas9 RNA injections in mouse zygotes. Dev Biol 2014; 393:3-9.

451. Lewis GS, Thatcher WW, Bazer FW, Curl JS. Metabolism of arachidonic acid in vitro by bovine blastocysts and endometrium. Biol Reprod 1982; 27:431-439.

452. Sagini K, Costanzi E, Emiliani C, Buratta S, Urbanelli L. Extracellular Vesicles as Conveyors of Membrane-Derived Bioactive Lipids in Immune System. Int J Mol Sci 2018; 19.

453. Roberts RM, Cross JC, Leaman DW. Interferons as hormones of pregnancy. Endocr Rev 1992; 13:432-452.

454. Kliewer SA, Lenhard JM, Willson TM, Patel I, Morris DC, Lehmann JM. A prostaglandin $\mathrm{J} 2$ metabolite binds peroxisome proliferator-activated receptor gamma and promotes adipocyte differentiation. Cell 1995; 83:813819.

455. Alhouayek M, Muccioli GG. COX-2-derived endocannabinoid metabolites as novel inflammatory mediators. Trends Pharmacol Sci 2014; 35:284-292.

456. Kozak KR, Crews BC, Morrow JD, Wang LH, Ma YH, Weinander R, Jakobsson PJ, Marnett LJ. Metabolism of the endocannabinoids, 2arachidonylglycerol and anandamide, into prostaglandin, thromboxane, and prostacyclin glycerol esters and ethanolamides. J Biol Chem 2002; 277:44877-44885.

457. O'Sullivan SE. An update on PPAR activation by cannabinoids. $\mathrm{Br} \mathrm{J}$ Pharmacol 2016; 173:1899-1910.

458. Sun X, Dey SK. Aspects of endocannabinoid signaling in periimplantation biology. Mol Cell Endocrinol 2008; 286:S3-11.

459. Wang $\mathrm{H}$, Xie $\mathrm{H}$, Dey SK. Endocannabinoid signaling directs periimplantation events. AAPS J 2006; 8:E425-432.

460. Wooding FB. Current topic: the synepitheliochorial placenta of ruminants: binucleate cell fusions and hormone production. Placenta 1992; 13:101113.

461. Klisch K, Hecht W, Pfarrer C, Schuler G, Hoffmann B, Leiser R. DNA content and ploidy level of bovine placentomal trophoblast giant cells. Placenta 1999; 20:451-458.

462. Klisch K, Thomsen PD, Dantzer V, Leiser R. Genome multiplication is a generalised phenomenon in placentomal and interplacentomal trophoblast giant cells in cattle. Reprod Fertil Dev 2004; 16:301-306.

463. Daniels TF, Killinger KM, Michal JJ, Wright RW, Jr., Jiang Z. Lipoproteins, cholesterol homeostasis and cardiac health. Int J Biol Sci 2009; 5:474-488.

464. Rader DJ. Molecular regulation of HDL metabolism and function: implications for novel therapies. J Clin Invest 2006; 116:3090-3100. 
465. Yvan-Charvet $\mathrm{L}$, Wang N, Tall AR. Role of HDL, ABCA1, and ABCG1 transporters in cholesterol efflux and immune responses. Arterioscler Thromb Vasc Biol 2010; 30:139-143.

466. Cockerill GW, Rye KA, Gamble JR, Vadas MA, Barter PJ. High-density lipoproteins inhibit cytokine-induced expression of endothelial cell adhesion molecules. Arterioscler Thromb Vasc Biol 1995; 15:1987-1994.

467. Gordon EM, Figueroa DM, Barochia AV, Yao X, Levine SJ. High-density Lipoproteins and Apolipoprotein A-I: Potential New Players in the Prevention and Treatment of Lung Disease. Front Pharmacol 2016; 7:323.

468. Khanal P, Pandey D, Binti Ahmad S, Safayi S, Kadarmideen HN, Olaf Nielsen M. Differential impacts of late gestational over-and undernutrition on adipose tissue traits and associated visceral obesity risk upon exposure to a postnatal high-fat diet in adolescent sheep. Physiol Rep 2020; 8:e14359.

469. Juszczuk-Kubiak E, Bujko K, Cymer M, Wicinska K, Gabryszuk M, Pierzchala M. Effect of Inorganic Dietary Selenium Supplementation on Selenoprotein and Lipid Metabolism Gene Expression Patterns in Liver and Loin Muscle of Growing Lambs. Biol Trace Elem Res 2016; 172:336-345.

470. Tanford C. The hydrophobic effect and the organization of living matter. Science 1978; 200:1012-1018.

471. Tang C, Vaughan AM, Oram JF. Janus kinase 2 modulates the apolipoprotein interactions with $A B C A 1$ required for removing cellular cholesterol. J Biol Chem 2004; 279:7622-7628.

472. Vaughan AM, Oram JF. ABCA1 redistributes membrane cholesterol independent of apolipoprotein interactions. J Lipid Res 2003; 44:13731380.

473. Nyalwidhe J, Burch T, Bocca S, Cazares L, Green-Mitchell S, Cooke M, Birdsall P, Basu G, Semmes OJ, Oehninger S. The search for biomarkers of human embryo developmental potential in IVF: a comprehensive proteomic approach. Mol Hum Reprod 2013; 19:250-263.

474. Jin F, Hagemann N, Sun L, Wu J, Doeppner TR, Dai Y, Hermann DM. Highdensity lipoprotein (HDL) promotes angiogenesis via S1P3-dependent VEGFR2 activation. Angiogenesis 2018; 21:381-394.

475. Patel S, Di Bartolo BA, Nakhla S, Heather AK, Mitchell TW, Jessup W, Celermajer DS, Barter PJ, Rye KA. Anti-inflammatory effects of apolipoprotein A-I in the rabbit. Atherosclerosis 2010; 212:392-397.

476. Yvan-Charvet L, Pagler TA, Seimon TA, Thorp E, Welch CL, Witztum JL, Tabas I, Tall AR. ABCA1 and ABCG1 protect against oxidative stressinduced macrophage apoptosis during efferocytosis. Circ Res 2010; 106:1861-1869.

477. Parameswaran N, Patial S. Tumor necrosis factor-alpha signaling in macrophages. Crit Rev Eukaryot Gene Expr 2010; 20:87-103.

478. Lee RS, Wheeler TT, Peterson AJ. Large-format, two-dimensional polyacrylamide gel electrophoresis of ovine periimplantation uterine luminal fluid proteins: identification of aldose reductase, cytoplasmic actin, and 
transferrin as conceptus-synthesized proteins. Biol Reprod 1998; 59:743752.

479. Sakai-Kato K, Yoshida K, Takechi-Haraya Y, Izutsu KI. Physicochemical Characterization of Liposomes That Mimic the Lipid Composition of Exosomes for Effective Intracellular Trafficking. Langmuir 2020.

480. Fraher D, Sanigorski A, Mellett NA, Meikle PJ, Sinclair AJ, Gibert Y. Zebrafish Embryonic Lipidomic Analysis Reveals that the Yolk Cell Is Metabolically Active in Processing Lipid. Cell Rep 2016; 14:1317-1329.

481. Sezgin E, Schwille P. Fluorescence techniques to study lipid dynamics. Cold Spring Harb Perspect Biol 2011; 3:a009803.

482. Unlocking the lipid labyrinth. Nat Chem Biol 2010; 6:471.

483. Balla T, Szentpetery Z, Kim YJ. Phosphoinositide signaling: new tools and insights. Physiology (Bethesda) 2009; 24:231-244.

484. Balla T. Phosphoinositides: tiny lipids with giant impact on cell regulation. Physiol Rev 2013; 93:1019-1137.

485. Hannun YA, Obeid LM. Principles of bioactive lipid signalling: lessons from sphingolipids. Nat Rev Mol Cell Biol 2008; 9:139-150.

486. Fendt SM, Bell EL, Keibler MA, Olenchock BA, Mayers JR, Wasylenko TM, Vokes NI, Guarente L, Vander Heiden MG, Stephanopoulos G. Reductive glutamine metabolism is a function of the alpha-ketoglutarate to citrate ratio in cells. Nat Commun 2013; 4:2236.

487. Moffatt BA, Ashihara $\mathrm{H}$. Purine and pyrimidine nucleotide synthesis and metabolism. Arabidopsis Book 2002; 1:e0018. 


\section{VITA}

Eleanore O'Neil grew up in Federal Way, WA in a large family as one of four kids. She became interested in science from a young age. She dropped out of high school at 16 years old and enrolled into college at Bard College at Simon's Rock in Great Barrington, MA. While there, Eleanore worked in a veterinary clinic and had her first experience in a research lab studying the biodiversity of fish populations in a local stocked lake. Eleanore transferred to Cornell University in the fall of 2012 to pursue her Bachelor's Degree in Animal Sciences. While there, she worked in the lab of Dr. Xingen Lei and completed an Honor's Thesis in animal nutrition feeding defatted microalgae to broiler chicks to create a healthier meat product. During her time at Cornell, Eleanore fostered a passion for reproductive and developmental biology. She graduated Cum Laude with a Distinction in Research in 2015. Eleanore joined the lab of Dr. Thomas Spencer in August of 2015 to start her Ph.D. investigating early pregnancy events using sheep as a model organism. During her time there, she attended several national conferences. She was accepted to the Frontiers in Reproduction course in 2020 which was cancelled due to the Covid-19 pandemic. Following the completion of her Ph.D., she will join the lab of Dr. Blanche Capel at Duke University to study sexual differentiation and developmental biology and plans for a career in academia. 